\title{
EXPERIMENTAL INVESTIGATION OF ACTIVE WINGTIP VORTEX CONTROL USING SYNTHETIC JET ACTUATORS
}

\author{
A Thesis \\ presented to \\ the Faculty of California Polytechnic State University \\ San Luis Obispo \\ In Partial Fulfillment \\ of the Requirements for the Degree \\ Master of Science in Aerospace Engineering
}

by

Peter J. Sudak

August 2014 
(C) 2014

Peter J. Sudak

ALL RIGHTS RESERVED 


\section{COMMITTEE MEMBERSHIP}

TITLE:

AUTHOR:

DATE SUBMITTED:

COMMITTEE CHAIR:

COMMITTEE MEMBER:

COMMITTEE MEMBER:

COMMITTEE MEMBER:
Experimental Investigation of Active Wingtip Vortex Control Using Synthetic Jet Actuators

Peter J. Sudak

August 2014

Jin Tso, Ph.D.

Professor of Aerospace Engineering

Russell Westphal, Ph.D.

Professor of Mechanical Engineering

Rob McDonald, Ph.D.

Associate Professor of Aerospace Engineering

Faysal Kolkailah, Ph.D.

Professor of Aerospace Engineering 


\begin{abstract}
Experimental Investigation of Active Wingtip Vortex Control using Synthetic Jet Actuators

Peter J. Sudak
\end{abstract}

An experiment was performed in the Cal Poly Mechanical Engineering $2 \times 2 \mathrm{ft}$ wind tunnel to quantify the effect of spanwise synthetic jet actuation (SJA) on the drag of a NACA 0015 semispan wing. The wing, which was designed and manufactured for this experiment, has an aspect ratio of 4.20 , a span of $0.427 \mathrm{~m}$ (16.813"), and is built around an internal array of piezoelectric actuators, which work in series to create a synthetic jet that emanates from the wingtip in the spanwise direction. Direct lift and drag measurements were taken at a Reynolds Number of 100,000 and 200,000 using a load cell/slider mechanism to quantify the effect of actuation on the lift and drag. It was found that the piezoelectric disks used in the synthetic jet actuators cause structural vibrations that have a significant effect on the aerodynamics of the NACA 0015 model. The experiment was performed in a way as to isolate the effect of vibration from the effect of the synthetic jet on the lift and drag. Lift and drag data was supported with pressure readings from 60 pressure ports distributed in rows along the span of the wing. Oil droplet flow visualization was also performed to understand the effect of SJA near the wingtip.

The synthetic jet and vibration had effects on the drag. The synthetic jet with vibration decreased the drag only slightly while vibration alone could decrease drag significantly from $11.3 \%$ at $\alpha=4^{\circ}$ to $23.4 \%$ at $\alpha=10^{\circ}$ and $\operatorname{Re}=100,000$. The lift was slightly increased with a slight increase due to the jet and showed a slight increase due to vibration. Two complete rows of pressure ports at $2 \mathrm{y} / \mathrm{b}=37.5 \%$ and $85.1 \%$ showed changes in lift due to actuation as well. The synthetic jet increased the lift near the wingtip at $2 \mathrm{y} / \mathrm{b}=85.1 \%$ and had little to no effect inboard at the $37.5 \%$ location, hence, the synthetic jet changes the lift distribution on the wing. Oil flow visualization was used to support this claim. Without actuation, the footprint of the tip vortex was present on the upper surface of the wing. With actuation on, the footprint disappeared suggesting the vortex was pushed off the wingtip by the jet. It is possible that the increased lift with actuation can be caused by the vortex being pushed outboard.

Keywords: Active Flow Control, NACA 0015, Semispan Testing, Synthetic Jet, Piezoelectric Actuators, Design, Wingtip Vortex, Hot-wire. 


\section{ACKNOWLEDGMENTS}

First of all, I would like to thank my parents for supporting me not only while I worked on my Thesis but throughout my college career. I could not have made it without you and I cannot even express how grateful I am. Thank you very much. Acknowledgments

I would like to thank Dr. Tso for taking me on as his graduate student. Dr. Tso, thank you for always being readily available to offer your support and providing the resources I needed to complete my Thesis. It was a pleasure having you as an advisor.

I would like to give a big thank you to Dr. Westphal for letting me use the Mechanical Engineering wind tunnel while the Aero tunnel was being repaired. Also, thank you for always being willing to share your expert advice and letting me use all the cool equipment in the ME tunnel.

I would like to thank Dr. McDonald for serving on my committee and answering questions that I had. It was helpful to hear your perspective on various aspects of this project.

I would like to thank Dr. Kolkailah for accepting a position on my committee last minute after one member became unavailable. I hope you found my work interesting.

I would like to thank Cody Thompson for letting me use his machine shop and buying me supplies in a moment's notice whenever needed. Your assistance helped me finish this project it a "timely" manner

Finally yet importantly, I would like to thank Alan L'Esperance for his help in all aspects of this project from helping me with the manufacture of the wing to showing me how to use various pieces of test equipment that were used for this Thesis. 


\section{TABLE OF CONTENTS}

LIST OF TABLES $\quad$ ix

LIST OF FIGURES $\quad$ x

NOMENCLATURE Xiv

1 Introduction 1

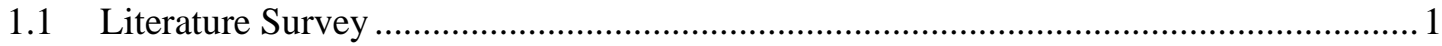

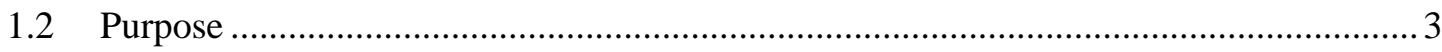

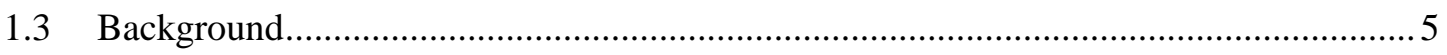

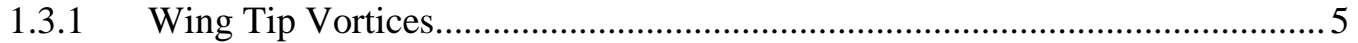

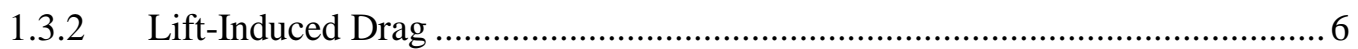

1.3.3 Zero-Net Mass-Flux Actuators ….................................................................... 6

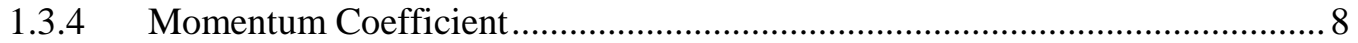

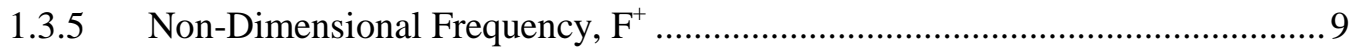

2 Design and Manufacture of Experiment 10

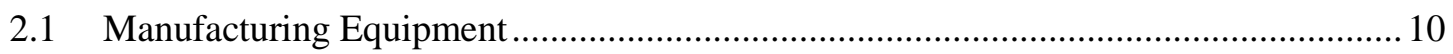

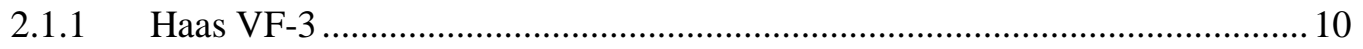

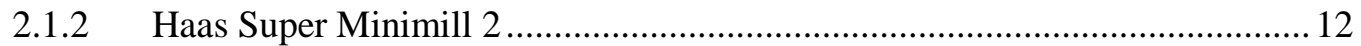

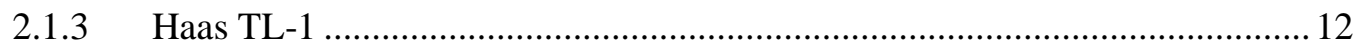

2.1.4 Universal Laser Systems VLS6.60 Laser cutter ........................................... 13

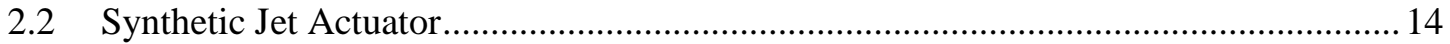

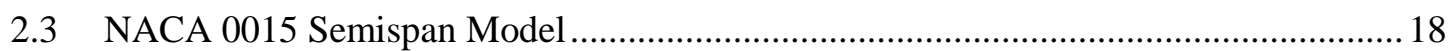

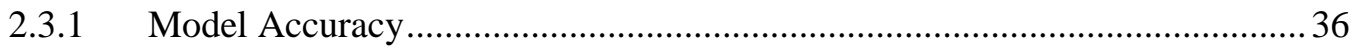

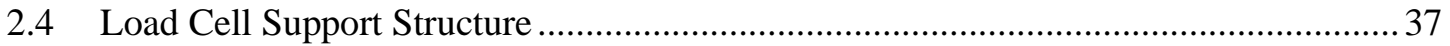

3 Experimental Apparatus $\quad 41$

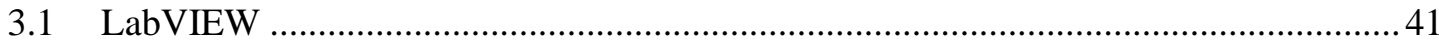

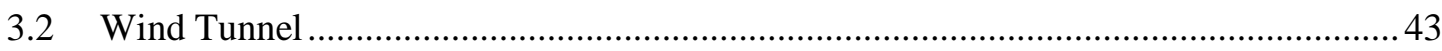

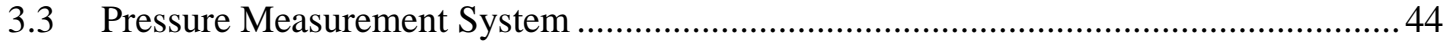




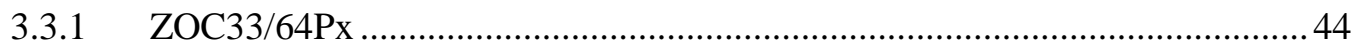

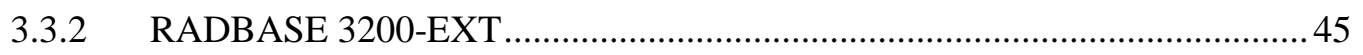

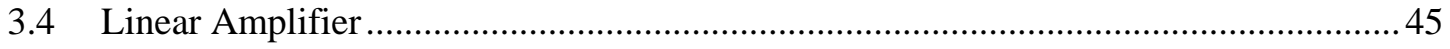

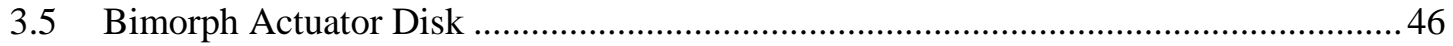

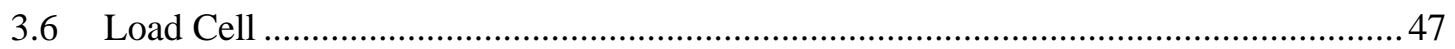

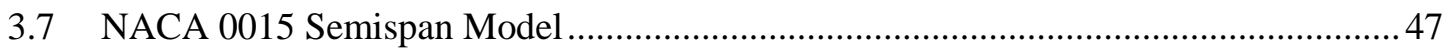

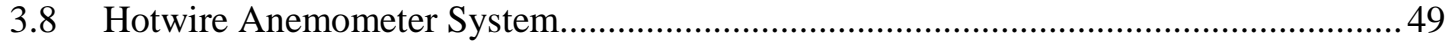

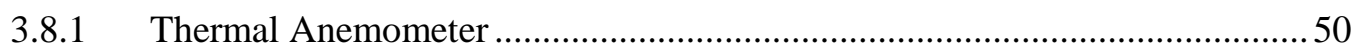

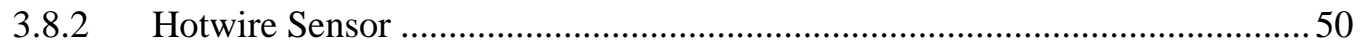

3.8.3 Automatic Velocity Calibrator..................................................................51

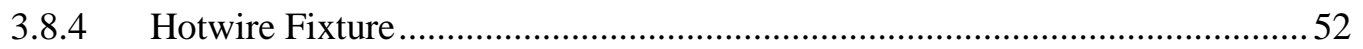

4 Analysis $\quad 54$

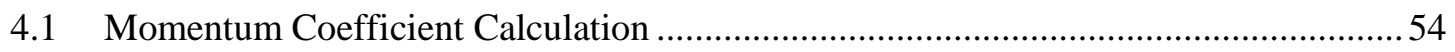

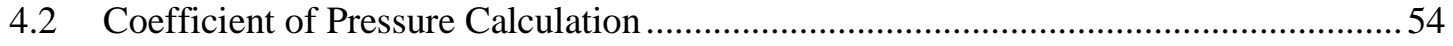

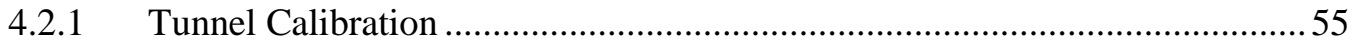

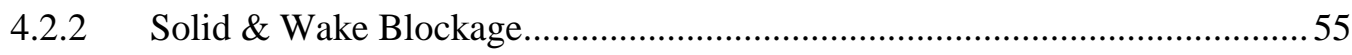

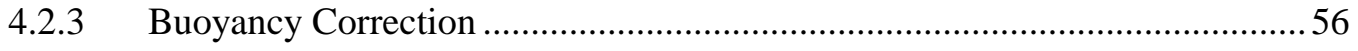

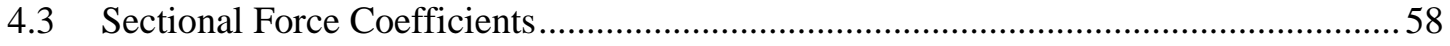

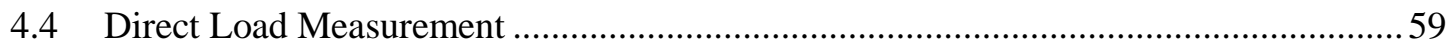

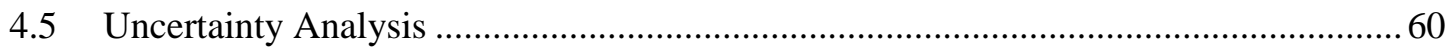

5 Experimental Procedure $\quad 62$

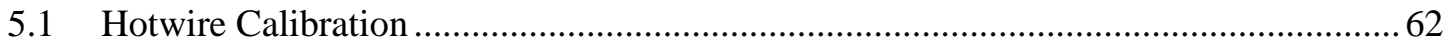

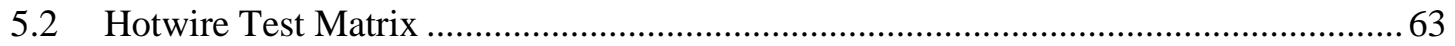

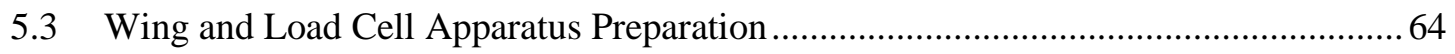

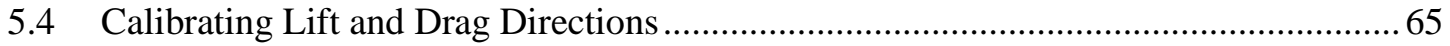

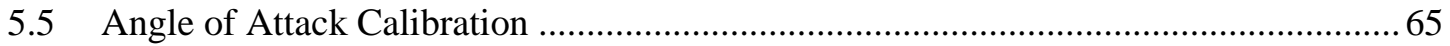

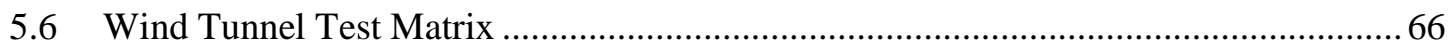

5.6.1 Additional Wind Tunnel Test Considerations ...............................................67

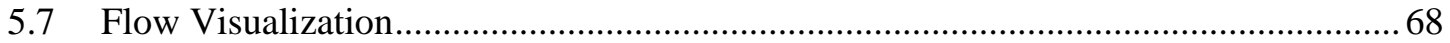




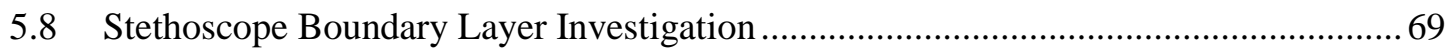

6 Results and Discussion $\quad 70$

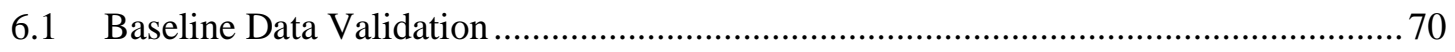

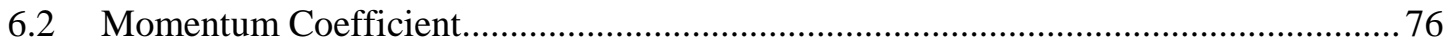

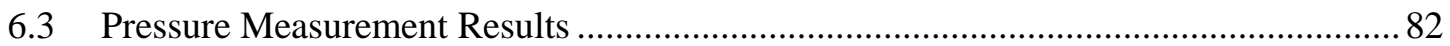

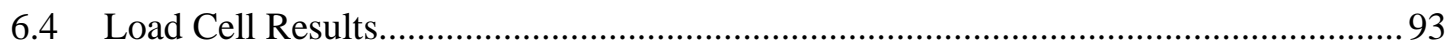

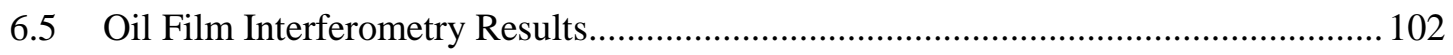

6.6 Stethoscope Boundary Layer Investigation Results .................................................. 103

7 Conclusion 105

$\begin{array}{ll}\text { Bibliography } & 107\end{array}$

Appendices

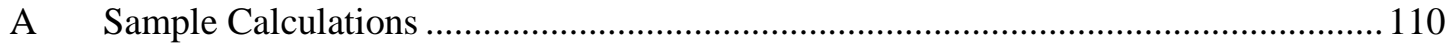

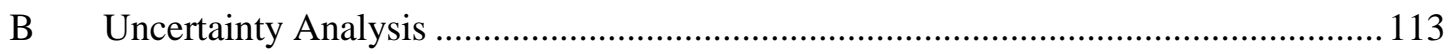

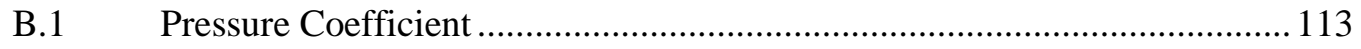

B.2 Sectional Lift Coefficient ....................................................................... 115

B.3 Lift and Drag Coefficient Error.............................................................. 117

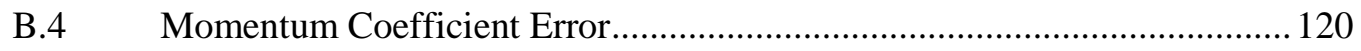

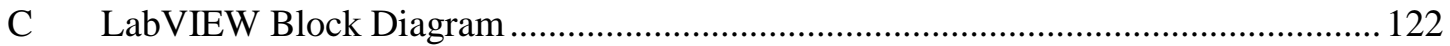




\section{LIST OF TABLES}

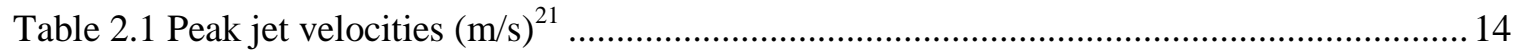

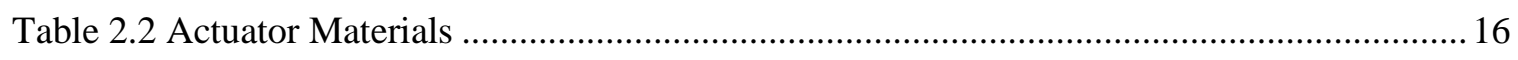

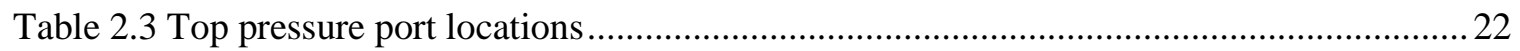

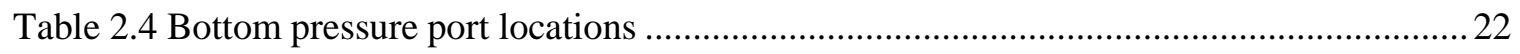

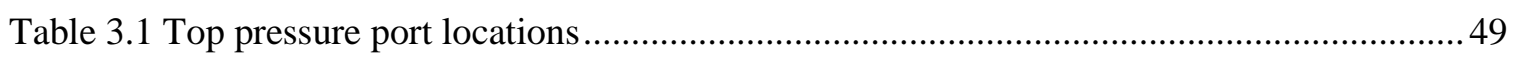

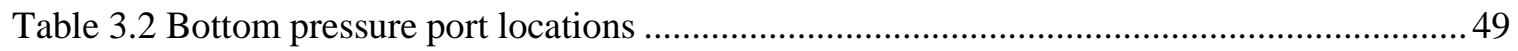

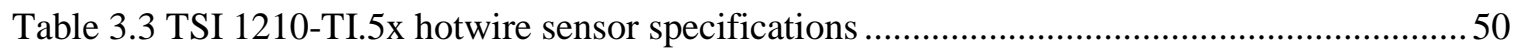

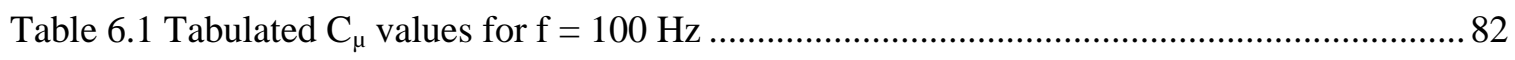

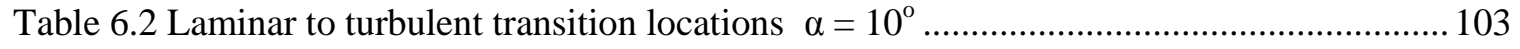




\section{LIST OF FIGURES}

Figure 1.1 Schematic of a piston cylinder synthetic jet actuator .............................................. 7

Figure 1.2 Schematic of a voice coil synthetic jet actuator......................................................

Figure 1.3 Schematic of a piezoelectric synthetic jet actuator .................................................... 8

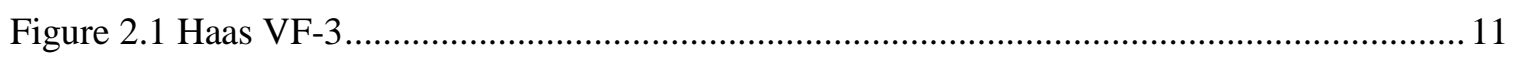

Figure 2.2 Wireless Probing System: Tool Setter (left) and Wireless Probe (right).................... 11

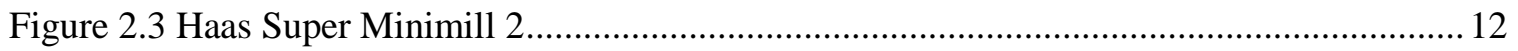

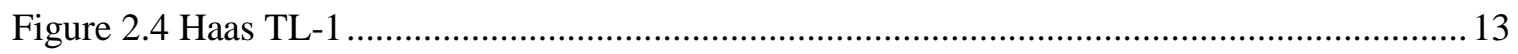

Figure 2.5 Universal Laser Systems VLS6.60 Laser Cutter ................................................... 14

Figure 2.6 Dimensioned drawing showing half of an actuator casing ...................................... 15

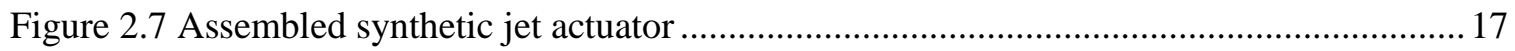

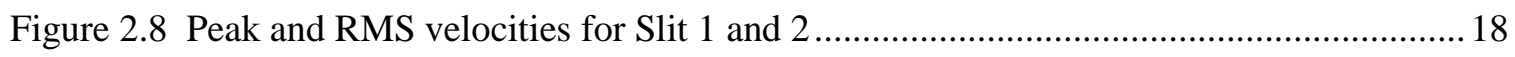

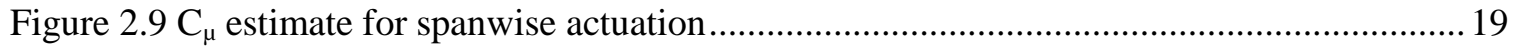

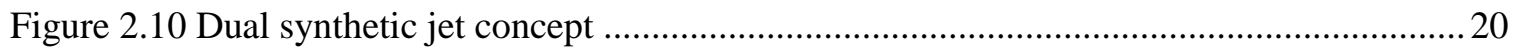

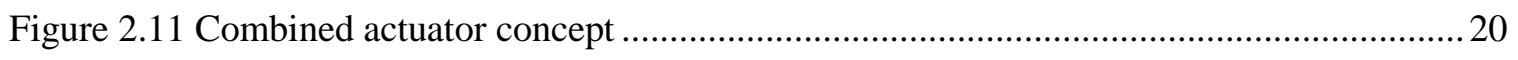

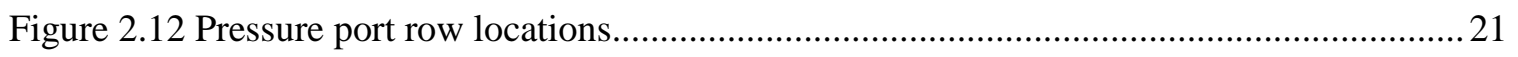

Figure 2.13 Top half of the wing showing internal details ........................................................24

Figure 2.14 Bottom half of the wing showing pressure surface .................................................26

Figure 2.15 Pressure surface actuator cover ……................................................................ 27

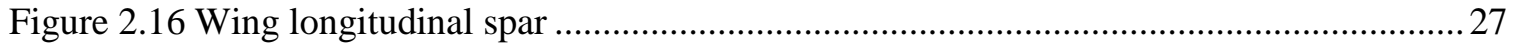

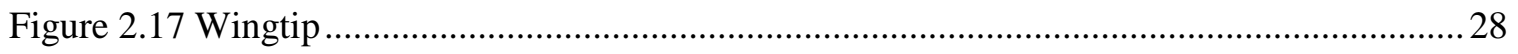

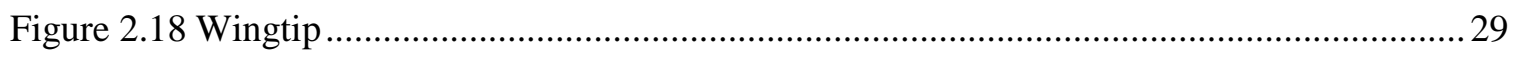

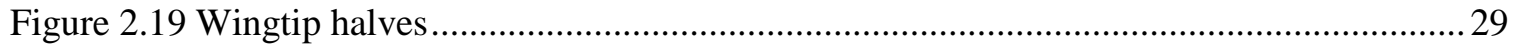

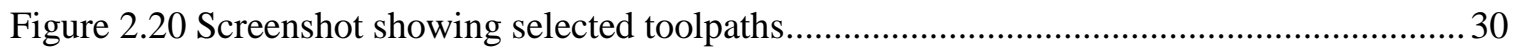

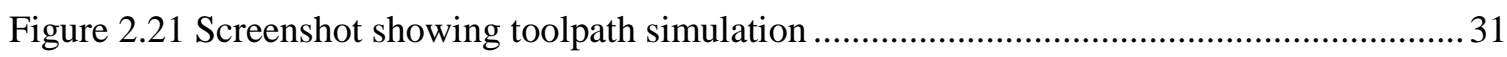

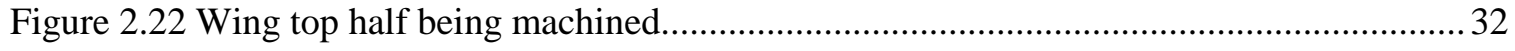

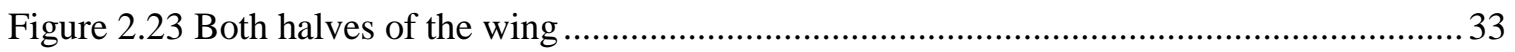

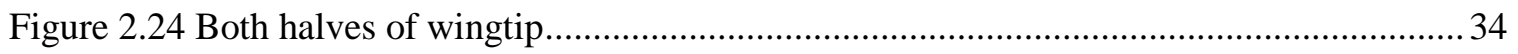




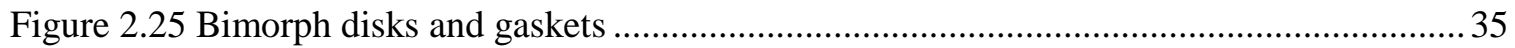

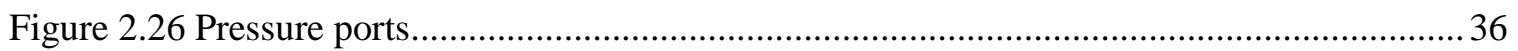

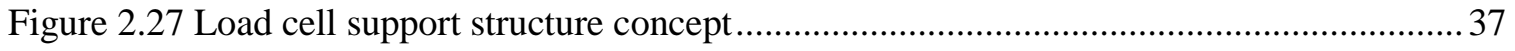

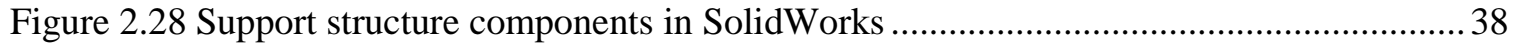

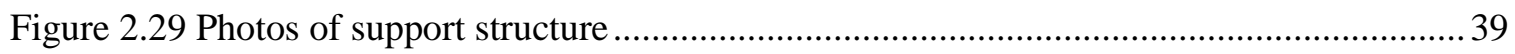

Figure 3.1 Schematic of Experimental Apparatus .................................................................... 41

Figure 3.2 LabVIEW Master.VI Screenshot........................................................................... 42

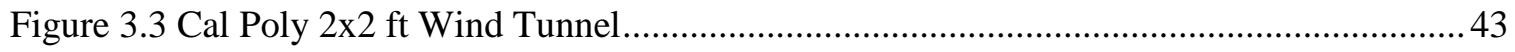

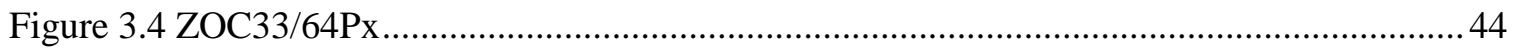

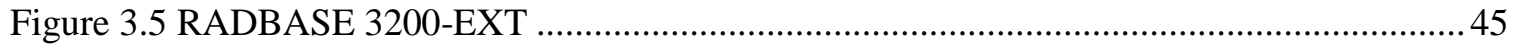

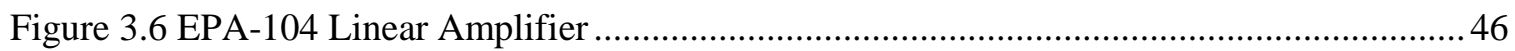

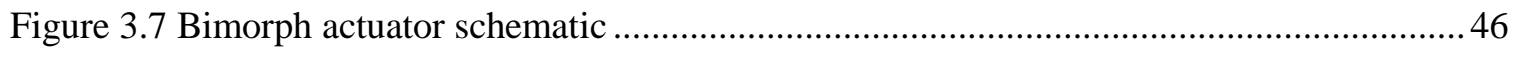

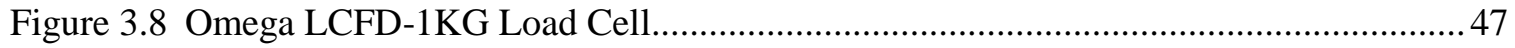

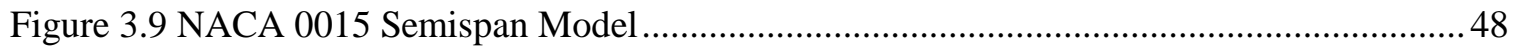

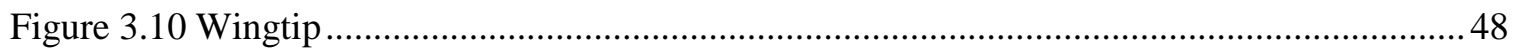

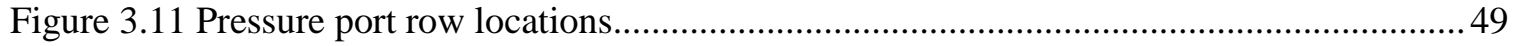

Figure 3.12 Model 1210 General Purpose Probe .........................................................................50

Figure 3.13 Automatic Velocity Calibrator System Schematic .................................................52

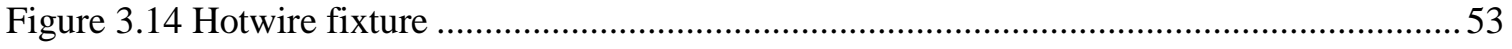

Figure 4.1 Wind tunnel lengthwise variation in static pressure, total pressure, and dynamic

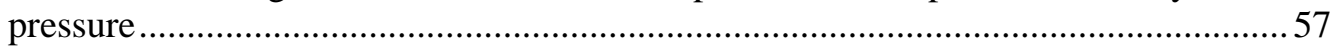

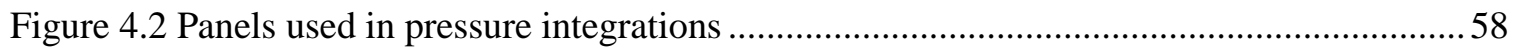

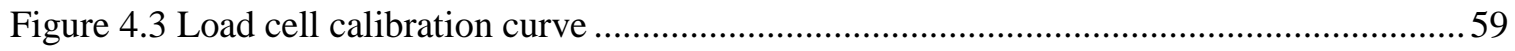

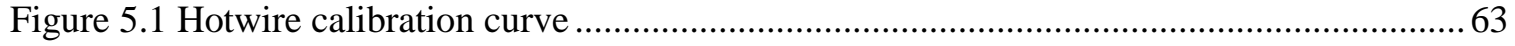

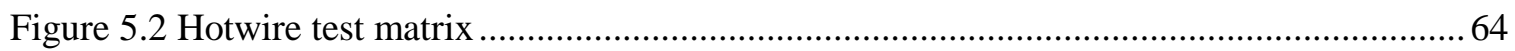

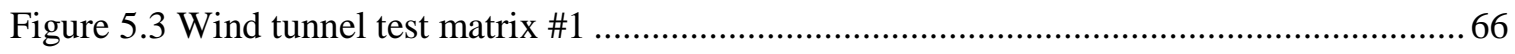

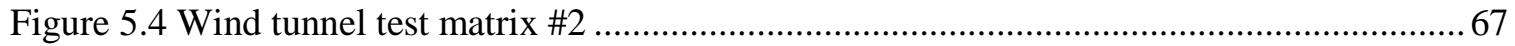

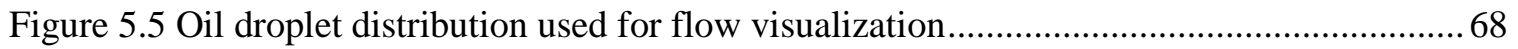

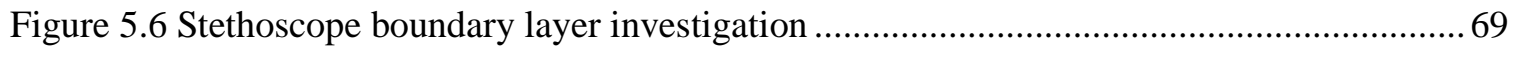

Figure 6.1 Rows A \& B baseline sectional lift compared to 2D NACA 0015 airfoil data ...........71 
Figure 6.2 Rows A \& B baseline Cp distribution at $\mathrm{Re}=100,000$ compared to NACA 0015 infinite wing at $\mathrm{Re}=60,000$.

Figure 6.3 Baseline sectional lift compared to NACA 0015 non-tripped finite wing $(\mathrm{Re}=$ $500,000 \mathrm{AR}=4)$ at $\alpha=10^{\circ}$

Figure 6.4 Baseline total lift at $\mathrm{Re}=200,000$ compared to NACA 0015 non-tripped finite wing $(\mathrm{Re}=200,000, \mathrm{AR}=4)$

Figure 6.5 Baseline total drag compared against: 1) NACA 0015 finite wing total and induced drag at $\operatorname{Re}=200,000 \mathrm{AR}=3$ with transition strips. 2) NACA 0015 data from NACA Report $586^{27}$

Figure 6.6 Synthetic jet RMS velocity for a range of frequencies ............................................. 76

Figure 6.7 Synthetic jet instantaneous velocity profile …............................................................ 77

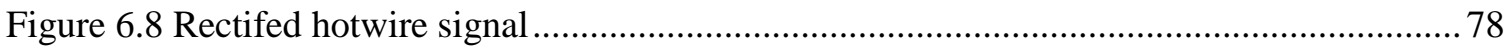

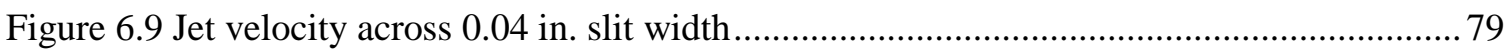

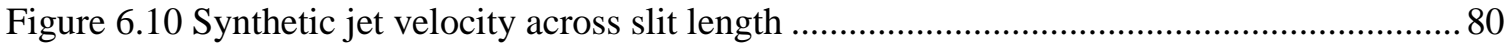

Figure 6.11 Change in RMS velocity with piezoelectric disk driving signal amplitude ............... 80

Figure 6.12 Momentum coefficient expected at various Reynolds numbers ............................... 81

Figure 6.13 Sectional lift coefficient comparison for baseline and actuated cases ........................ 83

Figure 6.14 Sectional lift coefficient comparison isolating the effect of vibration........................ 84

Figure 6.15 Sectional lift coefficient comparison isolating the effect of the synthetic jet............. 85

Figure 6.16 Row $\mathrm{F}$ suction surface $\mathrm{Cp}$ distribution at $2 \mathrm{y} / \mathrm{b}=0.93$ showing effect of wing

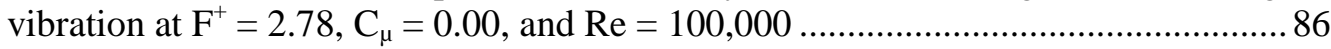

Figure 6.17 Row D suction surface $\mathrm{Cp}$ distribution at $2 \mathrm{y} / \mathrm{b}=0.85$ showing effect of wing

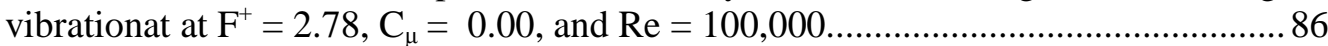

Figure 6.18 Row $\mathrm{C}$ suction surface $\mathrm{Cp}$ distribution at $2 \mathrm{y} / \mathrm{b}=0.70$ showing effect of wing vibration at at $\mathrm{F}^{+}=2.78, \mathrm{C}_{\mu}=0.00$, and $\mathrm{Re}=100,000$

Figure 6.19 Row A suction surface $\mathrm{Cp}$ distribution at $2 \mathrm{y} / \mathrm{b}=0.38$ showing effect of wing vibration at at $\mathrm{F}^{+}=2.78, \mathrm{C}_{\mu}=0.00$, and $\mathrm{Re}=100,000 \ldots$

Figure 6.20 Row A suction surface $\mathrm{Cp}$ distribution at $2 \mathrm{y} / \mathrm{b}=0.38$ showing effect of wing vibration at at $\mathrm{F}^{+}=1.39, \mathrm{C}_{\mu}=0.00$, and $\mathrm{Re}=200,000$.

Figure 6.21 Row F suction surface $\mathrm{Cp}$ distribution at $2 \mathrm{y} / \mathrm{b}=0.93$ showing effect of wing the synthetic jet at at $\mathrm{F}^{+}=2.78, \mathrm{C}_{\mu}=1.10 \%$, and $\mathrm{Re}=100,000$.

Figure 6.22 Row D suction surface $C p$ distribution at $2 \mathrm{y} / \mathrm{b}=0.85$ showing effect of wing the synthetic jet at at $\mathrm{F}^{+}=2.78, \mathrm{C}_{\mu}=1.10 \%$, and $\mathrm{Re}=100,000$ 
Figure 6.23 Row $C$ suction surface $C p$ distribution at $2 y / b=0.70$ showing effect of wing the synthetic jet at at $\mathrm{F}^{+}=2.78, \mathrm{C}_{\mu}=1.10 \%$, and $\mathrm{Re}=100,000$.

Figure 6.24 Row A suction surface $\mathrm{Cp}$ distribution at $2 \mathrm{y} / \mathrm{b}=0.38$ showing effect of wing thesynthetic jet at at $\mathrm{F}^{+}=2.78, \mathrm{C}_{\mu}=1.10 \%$, and $\mathrm{Re}=100,000$.

Figure 6.25 Row $\mathrm{F}$ suction surface $\mathrm{Cp}$ distribution at $2 \mathrm{y} / \mathrm{b}=0.93$ showing effect of wing the synthetic jet at at $\mathrm{F}^{+}=1.39, \mathrm{C}_{\mu}=0.28 \%$, and $\mathrm{Re}=200,000$ 91

Figure 6.26 Sectional lift coefficient variation with driving signal amplitude 92

Figure 6.27 NACA 0015 lift curve for actuated and unactuated cases 94

Figure 6.28 NACA 0015 lift curve showing the isolated effect of vibration 94

Figure 6.29 NACA 0015 lift curve showing the isolated effect of the synthetic jet .95

Figure 6.30 NACA 0015 drag curve for actuated and unactuated cases .96

Figure 6.31 NACA 0015 drag curve showing the isolated effect of vibration .96

Figure 6.32 NACA 0015 drag curve showing the isolated effect of the synthetic jet .97

Figure 6.33 NACA 0015 L/D curve for actuated and unactuated cases .98

Figure 6.34 NACA 0015 L/D showing the isolated effect of vibration......................................98

Figure 6.35 NACA 0015 L/D curve showing the isolated effect of the synthetic jet ...................99

Figure 6.36 NACA $0015 \mathrm{C}_{\mathrm{D}}$ vs. $\mathrm{C}_{\mathrm{L}}{ }^{2}$ curve for actuated and unactuated cases ........................... 100

Figure 6.37 NACA $0015 \mathrm{C}_{\mathrm{D}}$ vs. $\mathrm{C}_{\mathrm{L}}{ }^{2}$ curve showing the isolated effect of vibration................... 100

Figure 6.38 NACA $0015 \mathrm{C}_{\mathrm{D}}$ vs. $\mathrm{C}_{\mathrm{L}}{ }^{2}$ curve showing the isolated effect of the synthetic jet........ 101

Figure 6.39 Oil film interferometry on suction surface $\left(\operatorname{Re}=100,000 \alpha=10^{\circ}\right)$..................... 102

Figure 6.40 Oil film interferometry on tip surface $\left(\operatorname{Re}=100,000 \alpha=10^{\circ}\right)$. (a) Actuators off.

(b) Actuators on slit uncovered.......................................................................... 103

Figure 7.1 Error bars for Rows A \& B: Re = 100,000, $\alpha=10$ deg......................................... 115

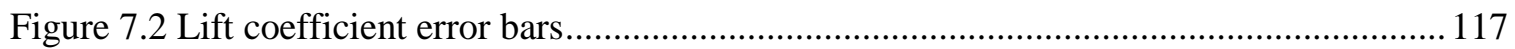

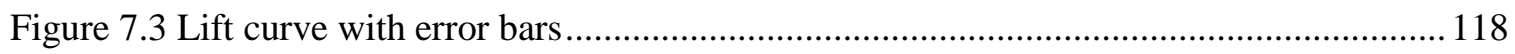

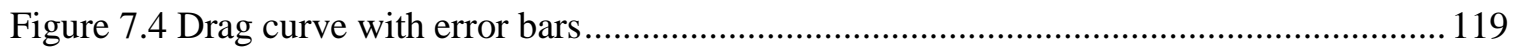

Figure 7.5 Drag curve with error bars showing error calculate using the standard deviation of repeat load cell readings 


\section{NOMENCLATURE}

\section{English Symbols}

\begin{tabular}{|c|c|}
\hline A & Area, $\mathrm{m}^{2}$ \\
\hline $\mathrm{b}$ & Wingspan, m \\
\hline $\mathrm{C}$ & Test section cross sectional area, $\mathrm{m}^{2}$ \\
\hline $\mathrm{C}_{\mathrm{d} 0}$ & Zero angle of attack drag coefficient \\
\hline $\mathrm{C}_{\mathrm{di}}$ & Induced drag coefficient \\
\hline $\mathrm{C}_{\mathrm{L}}$ & Lift coefficient \\
\hline $\mathrm{C}_{\mathrm{D}}$ & Drag coefficient ..................................................... $/\left(\mathrm{q}_{\infty} \mathrm{S}\right)$ \\
\hline $\mathrm{C}_{\mu}$ & Momentum coefficient............................................. $\rho_{j} A_{s} u_{r m s, j}^{2} /\left(q_{\infty} S\right)$ \\
\hline $\mathrm{C}_{\mathrm{P}}$ & Pressure coefficient ......................................... $\left(\mathrm{p}-\mathrm{p}_{\infty}\right) / \mathrm{q}_{\infty}$ \\
\hline $\mathrm{c}$ & Chord, m \\
\hline$c_{a}$ & Axial force coefficient \\
\hline $\mathrm{c}_{\mathrm{n}}$ & Normal force coefficient \\
\hline$c_{1}$ & Sectional lift coefficient ........................................... $\mathrm{L}^{\prime} / \mathrm{q}_{\infty} \mathrm{c}$ \\
\hline $\mathrm{c}_{\mathrm{d}}$ & Sectional drag coefficient ..................................... D'/ q $\mathrm{q}_{\infty} \mathrm{c}$ \\
\hline $\mathrm{D}$ & Drag, $\mathrm{N}$ \\
\hline $\mathrm{F}$ & Force, $\mathrm{N}$ \\
\hline $\mathrm{F}^{+}$ & Nondimensional frequency ................................ $\mathrm{fc} / \mathrm{u}_{\infty}$ \\
\hline $\mathrm{f}$ & Frequency, $\mathrm{Hz}$ \\
\hline $\mathrm{p}$ & Static pressure, $\mathrm{N} / \mathrm{m}^{2}$ \\
\hline $\mathrm{L}$ & Lift, $\mathrm{N}$ \\
\hline $\mathrm{q}_{\infty}$ & 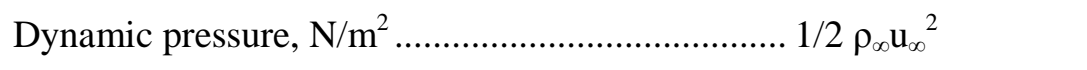 \\
\hline$S$ & 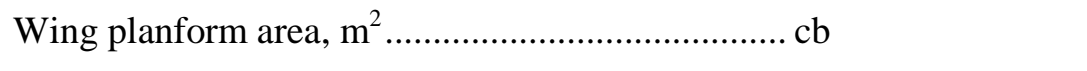 \\
\hline $\mathrm{Sr}$ & Strouhal number \\
\hline $\operatorname{Re}$ & Reynolds number ................................................ uc/v \\
\hline $\mathrm{u}$ & Velocity, m/s \\
\hline $\mathrm{V}$ & Voltage, Vpp \\
\hline $\mathrm{v}$ & Volume of wing, $\mathrm{m}^{3}$ \\
\hline $\mathrm{x}$ & Chord location starting at leading edge, $\mathrm{m}$ \\
\hline
\end{tabular}




\section{Greek Symbols}

$\alpha$

$\rho$

\section{Subscripts}

A

af

B

i

j

$\mathrm{p}$

q

RMS

$\mathrm{S}$

$\mathrm{sb}$

$\mathrm{t}$

tot

wb

$\varepsilon$

$\infty$

\section{Acronyms}

SJA

SJ

AFC

PFC

FSO

CFD
Angle of Attack, deg

density, $\mathrm{kg} / \mathrm{m}^{3}$

Actual

Airfoil

Buoyancy

An index

Jet

Static Pressure

Dynamic Pressure

Root mean squared

Slit

Solid blockage

Total

Total

Wake Blockage

Blockage

Freestream

Synthetic Jet Actuation

Synthetic Jet

Active Flow Control

Passive Flow Control

Full Scale Output

Computational Fluid Dynamics 


\section{Introduction}

With today's rising fuel prices, aircraft designers are looking for new ways to reduce the fuel consumption of aircraft. Reducing an aircraft's drag is one way to do this. It was estimated that a drag reduction of $10 \%$ on a single typical subsonic military aircraft would save 13 million gallons of fuel throughout its design life ${ }^{1}$. Various efforts have been geared towards achieving such drag reductions with a portion of these efforts focusing on wingtip devices. A properly designed wingtip device can modify a wing's lift distribution in a way that causes the wing to produce lift more efficiently. This improved efficiency decreases the lift-induced drag on the aircraft. Reducing the induced drag, which in certain cases accounts for about 30 percent of total drag for cruise ${ }^{2}$ and 80-90 percent for takeoff and landing ${ }^{3}$, would reduce the fuel consumption and could significantly reduce aircraft operating cost. In addition to reducing the induced drag, wingtip devices may have secondary benefits such as reducing the wake vortex hazard to trialing aircraft by weakening the trialing vortex ${ }^{4}$, and reducing carbon emissions by lowering fuel consumption ${ }^{5}$.

This document describes the wind tunnel test of an active "wingtip device" integrated into a NACA 0015 semispan model. This newly developed device uses an array of 8 piezoelectric disk actuators combined in series to create a synthetic jet that emanates out of the wingtip. It is believed that this synthetic jet device can be used to reduce the induced drag on a wing.

\subsection{Literature Survey}

The idea of a "wingtip device" to reduce induced drag has been around since the early $20^{\text {th }}$ century. Whitcomb, who coined the term winglet, seems to be the first to realize the potential induced drag benefits of these devices ${ }^{6}$. There have been several other winglet designs since then, notably the A310 tip fence, tip sails, and the vortex diffuser ${ }^{7}$. Although these devices generally provide a decrease in induced drag when designed correctly, they oftentimes do not 
"buy their way" onto aircraft. Reasons include structural issues, weight penalties, poor off design performance, and a net increase in other drag components such as profile drag ${ }^{8}$.

Active flow control (AFC) is being researched as an alternative and can eliminate many of the drawbacks associated with winglets. One benefit of AFC is that it is more versatile than passive flow control (PFC). This is because active devices can be scaled, turned on and off, and optimized throughout the flight envelope while passive devices cannot be modified as much and are generally optimized for one flight condition. Most AFC devices also have a minimal drag penalty since they are generally placed inside or near the wing surface. An early and widely studied wingtip AFC method involves steady blowing in the spanwise direction outboard from the wingtip. This generally results in an effective increase in span pushing the vortices outboard and diffusing the vortex. The diffused and displaced vortex produces a smaller downwash leading to an increase in lift and a decrease in induced drag for long-chord jets ${ }^{9}$. One issue with steady blowing AFC technology is that it requires a complicated ducting system to provide actuation. In addition, steady blowing has been shown to be impractical as the input required is greater than the increase in aerodynamic efficiency ${ }^{9}$. Synthetic jet or zero-net-mass-flux (ZNMF) actuation eliminates the ducting issue, as no air supply is needed. Studies have also shown that synthetic jets work better than steady blowing for separation control ${ }^{10}$ and that a synthetic jet entrains more fluid than a continuous jet ${ }^{11}$. This could mean that lower input is needed for a synthetic jet to achieve the same control authority and may prove synthetic jets more practical than steady actuation.

Limited research has been performed on the use of synthetic jets to control wingtip vortices although one study reported that synthetic jet actuation diffuses the vortex reducing core voritcity and maximum tangential velocity ${ }^{12}$. The paper compared the effect of synthetic jet actuation to the effect of steady blowing. Synthetic jet actuation and steady blowing showed similar effects for lower momentum coefficients but the synthetic jet shows a significantly more diffuse vortex 
for higher momentum coefficients. Recently, plasma actuation has been used to control the wingtip vortex providing promising results ${ }^{13,14}$. In a CFD simulation, a dielectric barrier discharge (DBD) actuator was able to block the crossflow around the wingtip at the origin of the vortex formation to produce a diffused vortex, decreased downwash, and a $20 \%$ increase in lift. Although promising, DBD actuators powerful enough to have these effects are still in development. Synthetic jets on the other hand have been shown to experimentally produce adequate excitation.

\subsection{Purpose}

The goal of this study is to conduct an experiment to determine if synthetic jet actuation is effective for reducing the induced drag on a finite wing. This study will expand on previous work $^{12}$ which showed the effect of synthetic jet actuation at several locations at the wingtip via particle image velocimetry (PIV). This previous paper concluded that synthetic jet actuation produces a diffuse vortex although did not provide any direct information about drag. The current study will conduct a similar experiment, using the most effective slit location from the previous study, and will provide direct drag measurements. This study will also test a wider range of angle of attack, $\mathrm{C} \mu$, and Reynolds number. $\mathrm{A} \mathrm{C}_{\mathrm{D}}$ vs $\mathrm{C}_{\mathrm{L}}{ }^{2}$ curve will be plotted with and without actuation to see if actuation has any effect on the induced drag. Several supporting data sets will be attained as well. Pressure data will be obtained through several pressure ports embedded in the model. This will be used to see if actuation is causing the lift coefficient to change near the wingtip as a change in the lift distribution should affect the induced drag. Oil droplet flow visualization will also be performed to see how actuation affects the local flowfield near the wingtip. Several things need to be completed to perform this study. First, a synthetic jet actuator needs to be designed and built that is suitable for active flow control. This actuator will need to be tested to ensure it provides sufficient actuation. Second, a compact semispan wing will have to be built which can contain these actuators and can fit inside the Cal Poly $2 \times 2 \mathrm{ft}$. subsonic wind 
tunnel. Sensors will have to be integrated into the model to measure drag and see if any decrease in induced drag is present. One requirement of the model is that it needs to be able to provide suction surface as well as tip actuation. This is because the model will be used for two different Thesis' (more information on this in section 4). Tip actuation will be used for this Thesis. Finally, the model will be tested in a wind tunnel to see if synthetic jet actuation from the wingtip provides any reduction in induced drag.

In summary, the goals of the current study are:

1. Design, build, and test a synthetic jet actuator

2. Design and build a NACA 0015 semispan wing model containing synthetic jet actuators that can provide suction surface, spanwise tip actuation, and a way to measure drag.

3. Expand upon a previous effort ${ }^{12}$ to control wingtip vortices via synthetic jet actuators. This will be done by conducting a wind tunnel experiment using the NACA 0015 semispan model with synthetic jet actuation at the wingtip, measuring the induced drag, and providing conclusions about the effect of synthetic jet actuation on induced drag. 


\subsection{Background}

\subsubsection{Wing Tip Vortices}

Tip vortices are formed at the terminating end of any lifting surface. They cause the air behind the lifting surface to swirl in a circular motion. Wingtip vortices, which form at the tips of aircraft wings, are the most notable occurrence of this and are widely researched by aerodynamicists, as they are detrimental to the aerodynamic efficiency of aircraft. Researchers often attempt to weaken, diffuse, or push these vortices outboard to reduce their effect on aerodynamic efficiency. Understanding the formation of the wingtip vortex is important in any effort to control it.

Green gives three distinctive and complimentary explanations for the occurrence of wingtip vortices ${ }^{15}$. The first and simplest method involves the pressure differential between the top and bottom wing surfaces. Due to the nature of the flow around a wing, the pressure on the upper (suction) surface is lower than the pressure on the lower (pressure) surface. This pressure differential accelerates the flow around the wingtip from the pressure surface to the suction surface combining with the streamwise velocity component creating a wingtip vortex. The second explanation involves the shear layer that exists near the wingtip. The freestream flow and the flow over the wing are not parallel which implies voritcity approaching the wing tips. This mechanism allows for the production of wingtip vortices of opposite sign on each side of the wing even without the production of lift, which actually does occur experimentally ${ }^{16}$. The third explanation involves the Helmholtz vortex laws. Here, the wingtip vortex is presented as a connection between the starting vortex and the bound vortex since vortex lines can never end in a fluid.

A more recent paper indicated that flow over the wingtip is more complicated, involving many different phenomena such as the interaction of multiple vortices, multiple separations and attachments, as well as being highly dependent on the wing geometry ${ }^{16}$. This paper contained 
flow over a square, untapered, and untwisted NACA 0012 wingtip (a square NACA 0015 is tested in this thesis) showing the formation of four vortices. These vortices eventually interact forming a highly unsteady vortex system that includes several secondary vortices.

\subsubsection{Lift-Induced Drag}

Induced drag is often the referred to as the price for producing lift. A basic way to understand induced drag is that the flow around the wingtip, aka wingtip vortex, contains a lot of translational and kinetic energy, which is wasted when the vortex is shed off the airplane. The engine has to work harder to overcome this wasted energy, hence to overcome the induced drag. A more systematic way to understand induced drag is as follows. The vortices shed from each tip create downwash or a downward velocity component ahead of the wing. This combines with the freestream velocity creating a local velocity around the wing that is canted downward. The local lift vector adjusts accordingly to be perpendicular to the local velocity producing a lift vector component in the freestream direction. This component can be thought of as the induced drag. According to Prandle's classical lifting-line theory the induced drag is proportional to the lift squared and inversely proportional to the aspect ratio. Therefore, according to this theory, the induced drag can be reduced by increasing the span or changing the lift on the wing.

\subsubsection{Zero-Net Mass-Flux Actuators}

A zero-net mass-flux actuator commonly known as the "synthetic jet" is formed by the periodic expulsion and ingestion of a fluid through an orifice. It is called a synthetic jet because there the jet is made or "synthesized" from the surrounding fluid. It ingests low speed fluid and ejects it at a higher speed adding momentum to the flow without adding mass. Synthetic jet actuators are commonly used for flow control applications, including separation control, mixing control, and combustion control to name a few. The most common synthetic jet actuator assemblies are piston cylinder, voice-coil magnet, or piezoelectric disk. The controlling 
mechanism causes flow to go in and out of an orifice creating a synthetic jet. A piston cylinder actuator is shown in Figure 1.1.

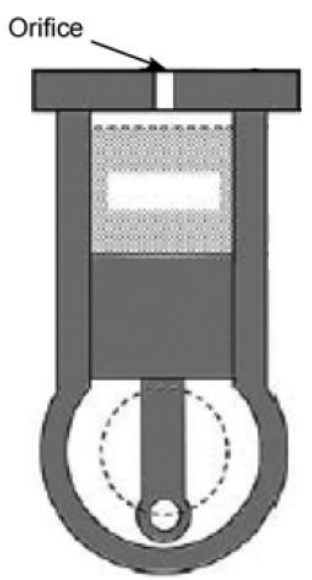

Figure 1.1 Schematic of a piston cylinder synthetic jet actuator ${ }^{17}$

Piston cylinder actuators can attain a high volumetric displacement for frequencies up to $200 \mathrm{~Hz}$ and generate very high cavity pressures ${ }^{18}$. This allows for supersonic velocities in both the ingestion and expulsion stroke. Piston cylinder synthetic jets are advantageous where high control authority is needed but only work well for relatively low frequencies and take up a lot of space. Another concept called the voice coil actuator is shown in Figure 1.2

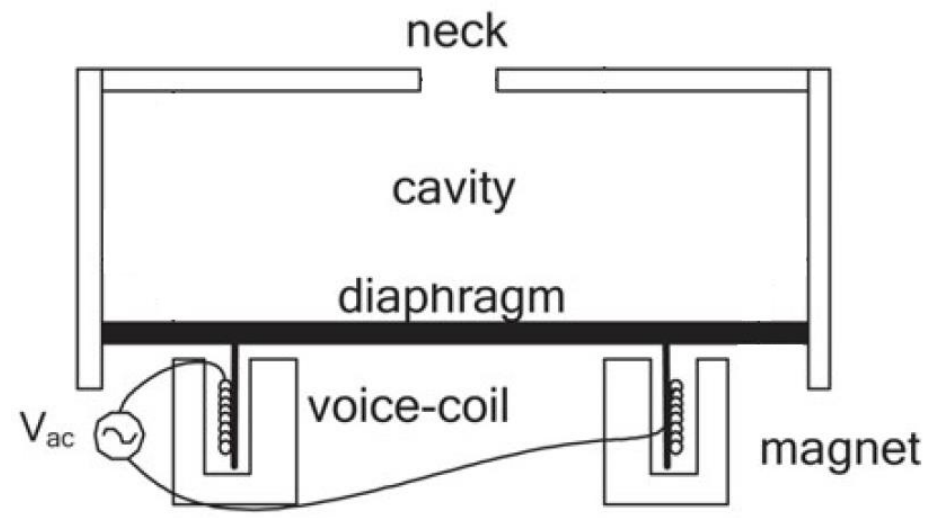

Figure 1.2 Schematic of a voice coil synthetic jet actuator ${ }^{19}$ 
The voice coil actuator is essentially a speaker attached to a cavity. It is capable of high frequency actuation and has a higher control authority than a piezoelectric synthetic jet but it is not as compact. A piezoelectric synthetic jet is shown in Figure 1.3.

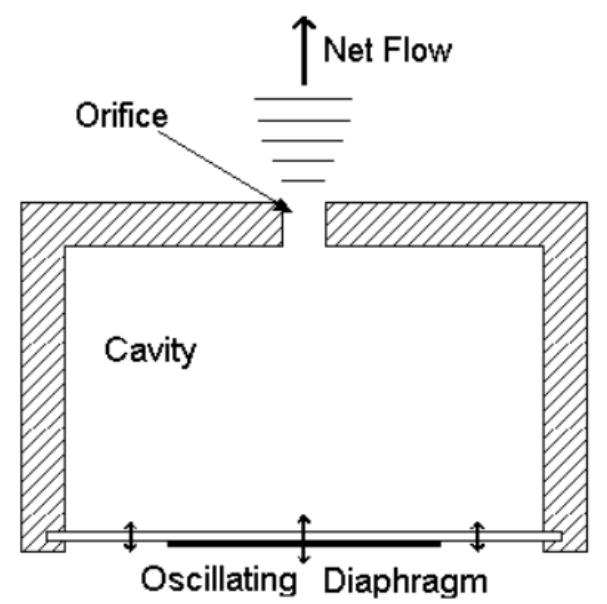

Figure 1.3 Schematic of a piezoelectric synthetic jet actuator ${ }^{20}$

The piezoelectric synthetic jet actuator is the simplest actuator of three. It consists of a piezoelectric disk, which acts as a diaphragm, and a cavity with a small orifice. Electric leads are attached to the disk on each side and an A/C current makes the disk oscillate forcing air in and out of the orifice. Although the piezoelectric synthetic jet has the lowest control authority, it is the easiest to build and is the most compact.

\subsubsection{Momentum Coefficient}

The momentum coefficient is a nondimensional parameter used to characterize the output of a flow control actuator. It is a common parameter in flow control studies and is used to gauge the effect of actuation on the performance parameters of interest. It is often defined by a steady component and an unsteady component.

$$
C_{\mu, t o t}=C_{\mu, \text { steady }}+C_{\mu, \text { unsteady }}
$$

For example, a synthetic jet would be defined purely by the unsteady component while steady blowing would be defined by the steady component. There are also schemes that use 
pulsed control, which would superimpose a steady mass flux into onto a zero-net-mass-flux device. This would be defined by both the steady and unsteady components. In this paper only the unsteady momentum coefficient is used which can be defined as:

$$
C_{\mu, \text { unsteady }}=\frac{\rho_{j} A_{s} u_{r m s, j}^{2}}{q_{\infty} S}
$$

Some papers use the peak jet velocity and some papers use the root mean squared (RMS) jet velocity for synthetic jets. There is also some discrepancy in how the RMS velocity is calculated. The RMS velocity can be calculated by using the velocity profile of the entire synthetic jet cycle (expulsion and ingestion) or just from the expulsion portion. This study uses the RMS velocity of the expulsion phase.

\subsubsection{Non-Dimensional Frequency, $\mathbf{F}^{+}$}

$\mathrm{F}^{+}$is a non-dimensional frequency describing oscillating flow mechanisms. It is the ratio of the time it takes flow to travel over the wing to the characteristic period of the actuator.

$$
F^{+}=\frac{f c}{u_{\infty}}
$$

In vortex control, $\mathrm{F}^{+}$is sometimes termed Strouhal Number and is used to measure phenomena such as vortex meandering, vortex instabilities, and shear layer instabilities. 


\section{Design and Manufacture of Experiment}

Synthetic jet actuators and an AFC test apparatus were not readily available for use. A preexisting AFC test apparatus would have to be bought from an independent source, which would be beyond the budget of this experiment. It was much more feasible to build the apparatus from scratch. The complexity and precision needed for the design were too high to do by hand. Therefore, everything was built using automated machinery

The design philosophy was to design for manufacturability. It was necessary to keep things as simple as possible because any added complexity leads to more difficulty in manufacturing. Because manufacturing is much more time consuming than the design process, essentially this philosophy reduces the time it takes to build everything.

\subsection{Manufacturing Equipment}

All of the manufacturing was done in the Cal Poly Mustang 60 machine shop, the Cal Poly Hangar, and the Cal Poly Aerospace Department's machine shop. It was necessary to receive the Blue tag certification from the Mustang 60 machine shop in order to be able to use the CNC machinery in this section. The prerequisites for this certification were 60 hours of logged shop use, taking a tour and passing a written test, and completion of a guided CNC project on each machine that would be used. The machines used for manufacturing this experiment are discussed in the following sections

\subsubsection{Haas VF-3}

The Haas VF3 is a CNC vertical milling center with a 40" x 20" x 25" travel. More specifics about the machine can be found at the Haas website. This is the only machine available on campus that was large enough to machine the NACA 0015 model. A picture of the VF-3 is shown in Figure 2.1 


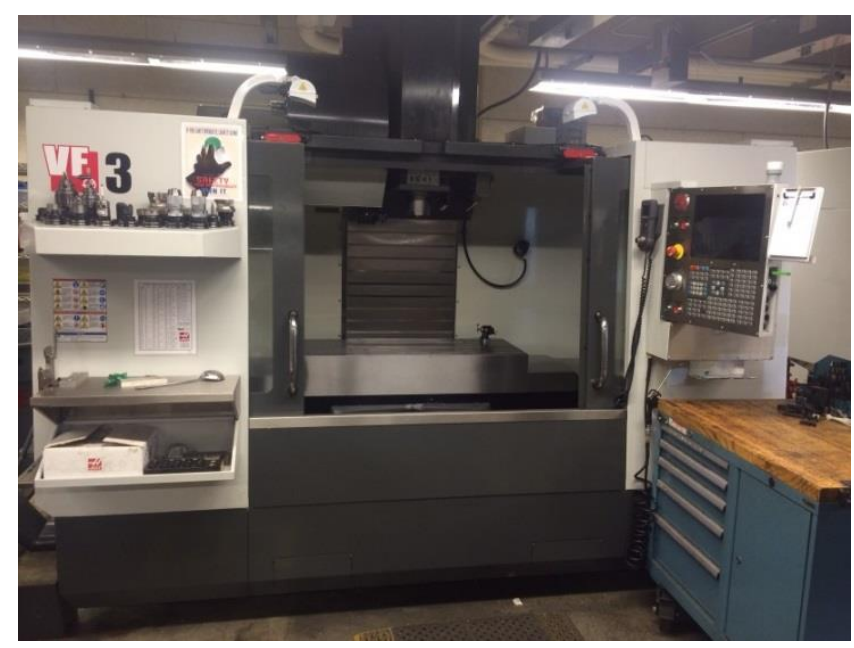

Figure 2.1 Haas VF-3

This machine has several upgrades that made it more convenient to machine the NACA 0015 including: a 15,000-rpm Spindle, a Side-Mount Tool Changer, a Chip Auger, and Wireless Intuitive Probing System (shown in Figure 2.2). A high-speed spindle was effective at reducing the already high 32 hour run time. A side-mount tool changer was necessary because a standard tool holder could not hold the 15 tools that were used for machining. The chip auger was coded to turn on automatically every hour of machining to remove chips and prevent them from clogging the coolant filtration system.
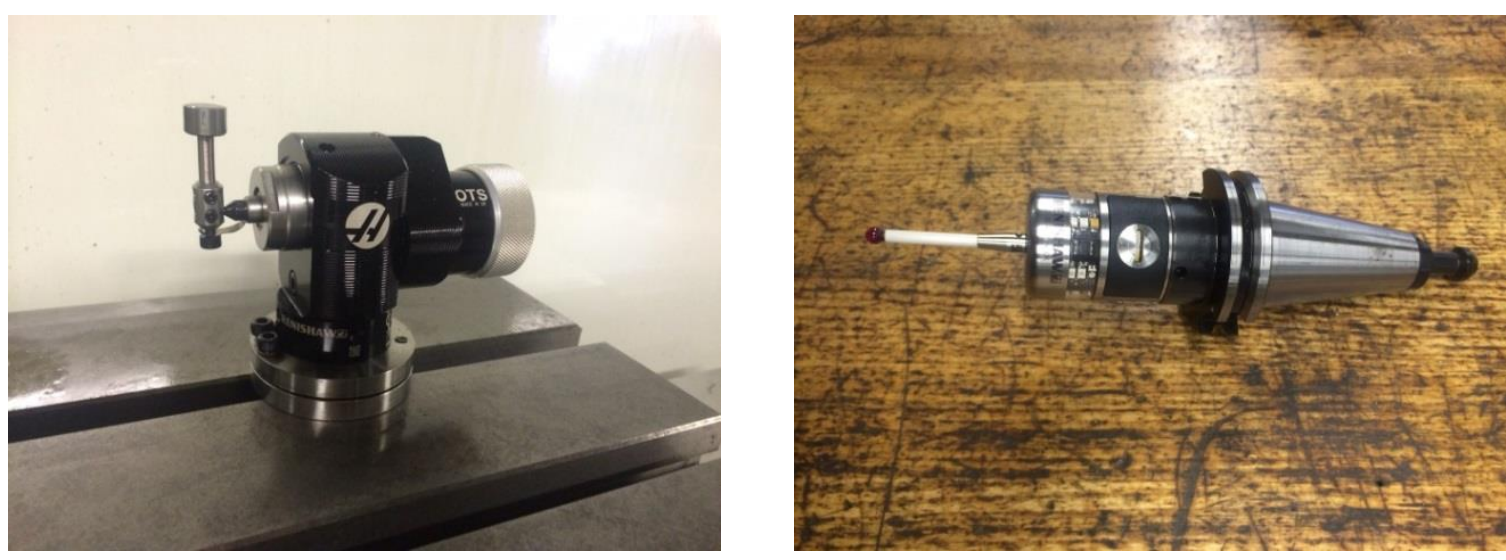

Figure 2.2 Wireless Probing System: Tool Setter (left) and Wireless Probe (right)

The wireless probing system was used as a quick and efficient way to set tool and part offsets and was very useful to reduce the setup time. The wireless probe would be used to touch off on the NACA 0015 giving the machine a reference of where the part is located relative to the 
spindle. Then, each tool would be touched off on the tool setter measuring its z-distance relative to the top of the NACA0015. The g-code for this machine was created using CAMWorks.

\subsubsection{Haas Super Minimill 2}

The Haas Super Minimill 2 is a CNC vertical milling center with a 20" x $16 "$ x 14 "travel, shown in Figure 2.3. More specifics about the machine can be found at the Haas website. This machine is smaller, easier to manage, and easier to clean than the VF-3. The VF-3 can do everything this machine can although it was much more convenient to work in this machine. The Minimill was used to build the smaller parts including the synthetic jet actuator, the wingtip, and some of the supporting structure. The VF-3 and the Minimill were often used simultaneously. The VF-3 would be machining large parts that take a long time. During this wait period, several small components could be machined on the Minimill. The mini also had similar upgrades to the VF-3 including the Wireless Intuitive Probing System. The g-code for this machine was created using CAMWorks

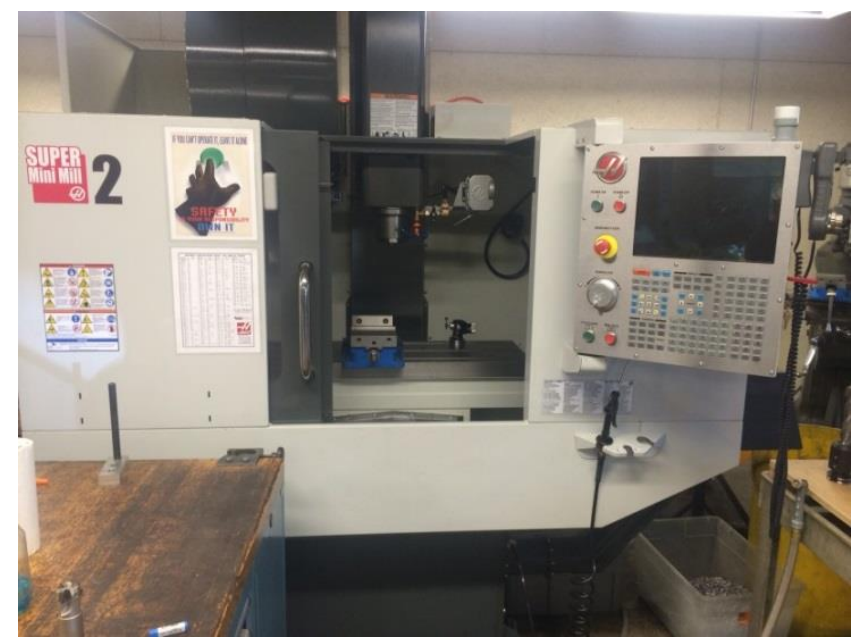

Figure 2.3 Haas Super Minimill 2

\subsubsection{Haas TL-1}

The Haas TL-1, shown in Figure 2.4, is a CNC/Manual Toolroom Lathe with a 16" x 29" max capacity. More specifics about the machine can be found at the Haas website. Several cylindrical components of the experimental apparatus had to be cut to precise lengths, drilled, and 
tapped. This machine was ideal for machining these components as it could be programmed to do these features automatically. The g-code for this machine was generated using the quick-code feature where simple operations such as drilling and tapping could be set using the TL-1 user interface.

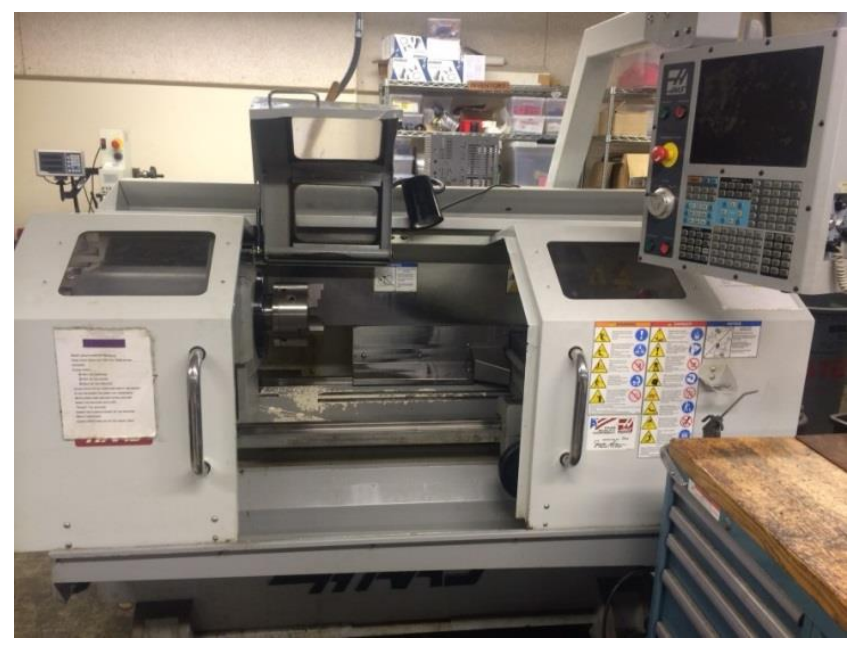

Figure 2.4 Haas TL-1

\subsubsection{Universal Laser Systems VLS6.60 Laser cutter}

The VLS6.60 laser cutter shown in Figure 2.5 is a high-powered laser used for cutting or engraving material. This laser uses a high power density allowing the laser to be focused on a very small spot for precision cutting and can cut objects up to 37 × 23 x 9 in. This laser cutter was used to cut gaskets for the actuator. It was necessary to have a thin, narrow, nonconductive gasket of specific dimensions to minimize the clamping area and insulate the piezoelectric disk. A gasket like this could not be purchased. The laser cutter proved efficient for cutting the delicate neoprene gasket material precisely. 


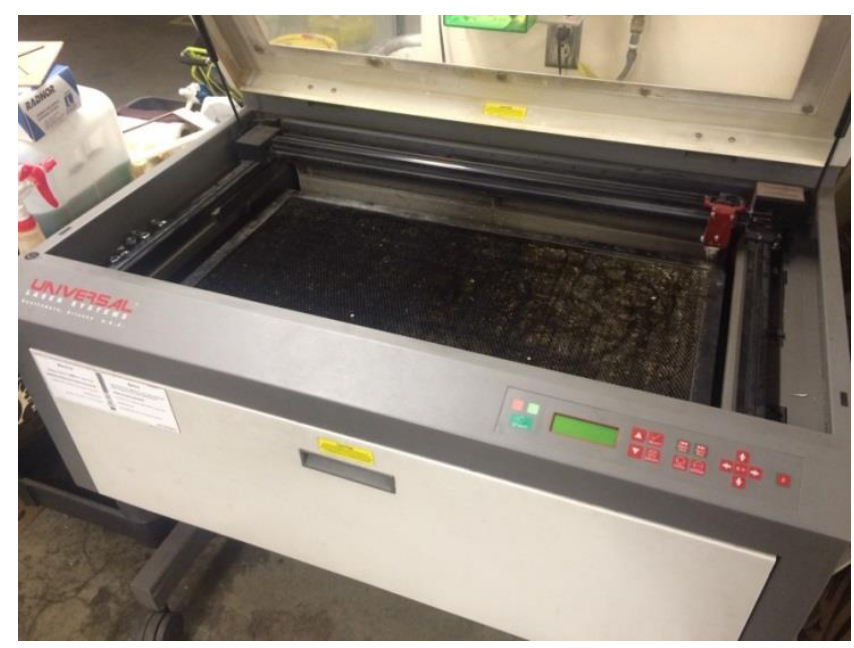

Figure 2.5 Universal Laser Systems VLS6.60 Laser Cutter

\subsection{Synthetic Jet Actuator}

In order to conduct the active flow control experiment, a synthetic jet actuator needed to be built. Several actuator concepts were considered (see section 1.3.3) but it all came down to getting the apparatus to fit inside the Cal Poly $2 \times 2$ wind tunnel and the piezoelectric actuator was chosen due to its compactness. Several types of piezoelectric materials were considered based on which could provide the highest peak velocity. A study was found which showed peak velocities for three materials using three different wave types ${ }^{21}$. Results of the study are shown in Table 2.1

Table 2.1 Peak jet velocities $(\mathrm{m} / \mathrm{s})^{21}$

\begin{tabular}{|c|c|c|c|}
\hline \multirow{2}{*}{ Diaphragm } & \multicolumn{3}{|c|}{ Waveform } \\
\cline { 2 - 4 } & Sine & Saw-tooth & Square \\
\hline Bimorph & $7 \pm 2$ & $35 \pm 6$ & $36 \pm 5$ \\
\hline Thunder & $5 \pm 2$ & $26 \pm 2$ & $27 \pm 2$ \\
\hline RFD & $6 \pm 2$ & $28 \pm 2$ & $32 \pm 2$ \\
\hline
\end{tabular}

From this, it can be seen that the Bimorph actuator produces the highest velocity output and that a square wave is the best driving signal. Another study was found which experimentally derived a model for the velocity of a Bimorph actuator ${ }^{22}$. This model is shown in equation 2.1.

$$
Y^{\prime}=18.53-14.53 F_{z}-3.29 D_{0}-2.96 C_{H}-4.72 P_{B}
$$


where $Y^{\prime}$ is the jet velocity, $F_{Z}$ is the driving waveform, $D_{0}$ is the orifice diameter, $C_{H}$ is the cavity height, and $P_{B}$ is the passive cavity pressure. The main point taken away from this is that it is necessary to keep the orifice diameter and cavity height as small as possible to maximize the jet velocity. The actuator dimensions will be chosen based on this data. The first step in building the actuator was to come up with a way to clamp the Bimorph disk and create a cavity to provide actuation. The idea was to make the cavity out of two near identical halves that would clamp down on the actuator. One of these halves was modeled using SolidWorks and is shown in Figure 2.6. The Bimorph disk will sit in the 0.045 lip shown in Figure 2.6. Two casing halves will then be screwed together clamping down on the disk.
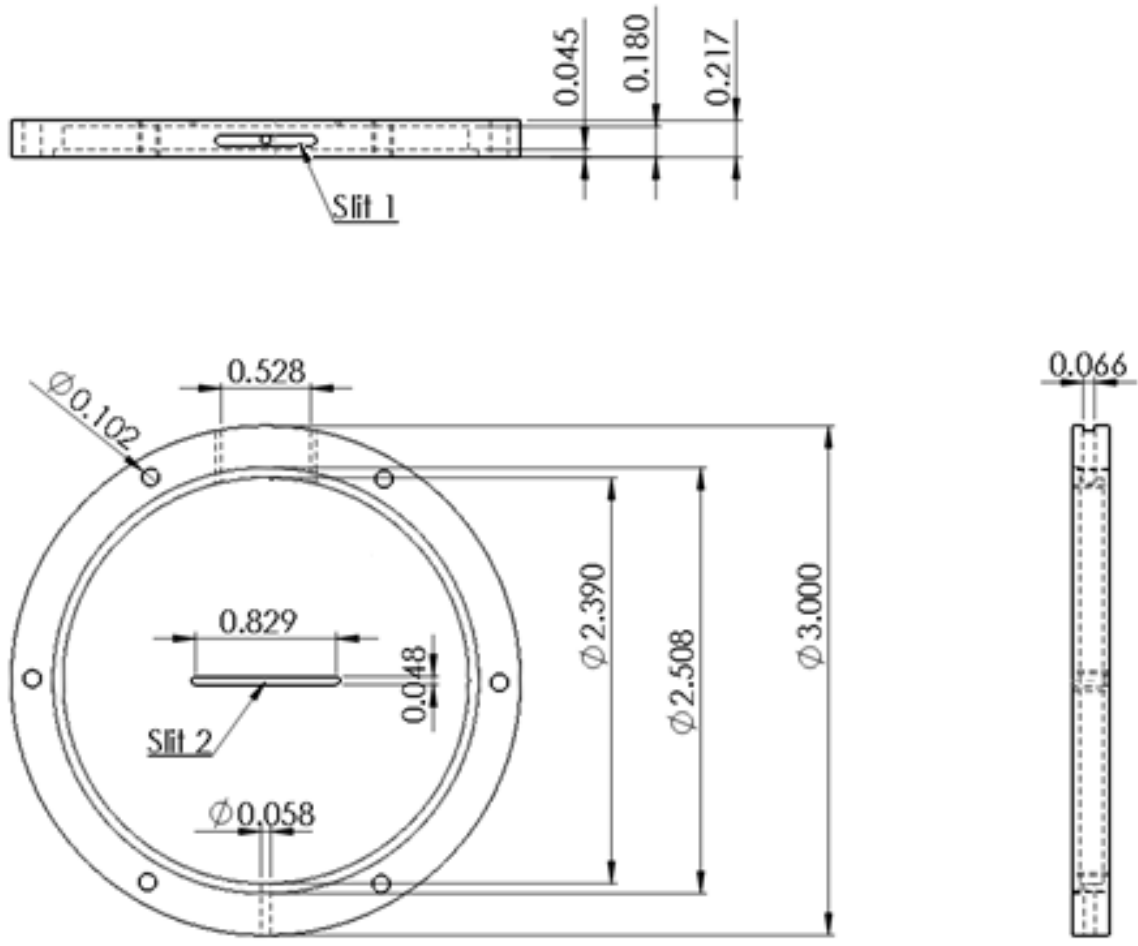

Figure 2.6 Dimensioned drawing showing half of an actuator casing

The next step was to choose the materials for the actuator. The material selection is summarized in Table 2.2. Cast acrylic was chosen for the casing because it is clear, stiff, insulating, and machinable. A stiff actuator was needed to prevent the actuators from bending under the stress of the wind in the wind tunnel breaking the stiff piezoelectric disks. A clear 
actuator was necessary to do quick visual damage assessments for things such as broken disks or disconnected leads. A maximum of 180 volts and $200 \mathrm{~mA}$ would be applied to the actuator so the electric insulation property was crucial for safety reasons. In addition, this actuator needed to be machined and acrylic is very machinable. The Bimorph material was chosen due to its velocity output as showed previously in this section. A gasket was necessary to provide a cushion for the Bimorph protecting it from being damaged as the casings clamp together on the disk's outer edge. Machine screws are used to clamp the actuator together. The electric leads are needed provide power to the actuator. The thinnest flexible wire that could handle 180 volts and $200 \mathrm{~mA}$ was used to prevent strain on the disk that could damage the actuator during operation.

Table 2.2 Actuator Materials

\begin{tabular}{|c|c|}
\hline Part & Material \\
\hline Casing & Cast Acrylic \\
\hline Actuator & Bimorph, Piezo Systems part no. \\
& T216-A4NO-573X \\
\hline Gasket & $\begin{array}{c}\text { 0.032" in thick 2.508 OD 2.390 ID } \\
\text { neoprene }\end{array}$ \\
\hline Machine Screws & $1 / 4$ 5-40, Stainless Steel \\
\hline Electric Leads & Continuous-Flex Miniature Wire 26 AWG \\
\hline
\end{tabular}

This casing was then manufactured using a Haas Minimill. The tools, tool paths, feeds, and speeds were all set using CAMWorks, which converted them to G-code to be read by the VF2. The piece of acrylic was squared off manually and then fixtured using a vise. A Haas Renishaw Probe was used to set the part offsets. A Haas wireless tool setter was used to set the tool lengths.

The gasket also had to be manufactured. A sketch of the gasket was made using Adobe Illustrator which was then printed to a Universal Laser Systems VLS6.60 Laser cutter. A Power of $55 \%$, speed of $10 \%$, and pulses per inch (PPI) of 600 were the laser settings used for cutting the gasket. In addition, the gasket was cut a bit larger than showed in Table 2.2 to account for the 
laser diameter. The actual OD and ID that were commanded to the laser are 2.512" and 2.386" respectively. Figure 2.7 shows the complete and assembled actuator. For the initial tests, electrical tape was used to hold the leads in place. Later on, the leads were soldered to the disk.

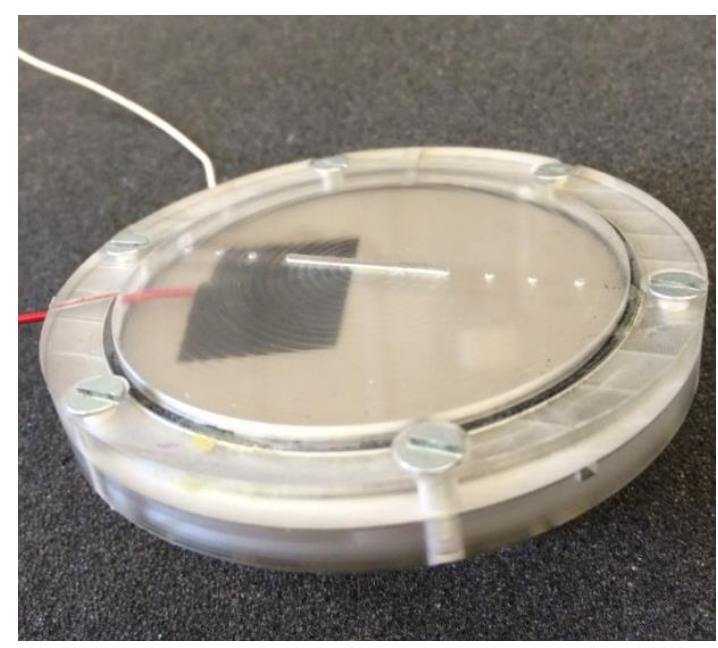

Figure 2.7 Assembled synthetic jet actuator

It was necessary to measure the velocity output of the actuator to get an estimate of $\mathrm{C}_{\mu}$ for use in the design of the NACA 0015 model. Slit 1 and slit 2 (shown in Figure 2.6) were tested because they were thought to represent the slit orientations used in the NACA 0015 model (see Section 2.3). The peak and RMS velocity profiles for each slit orientation are shown in Figure 2.8 . 


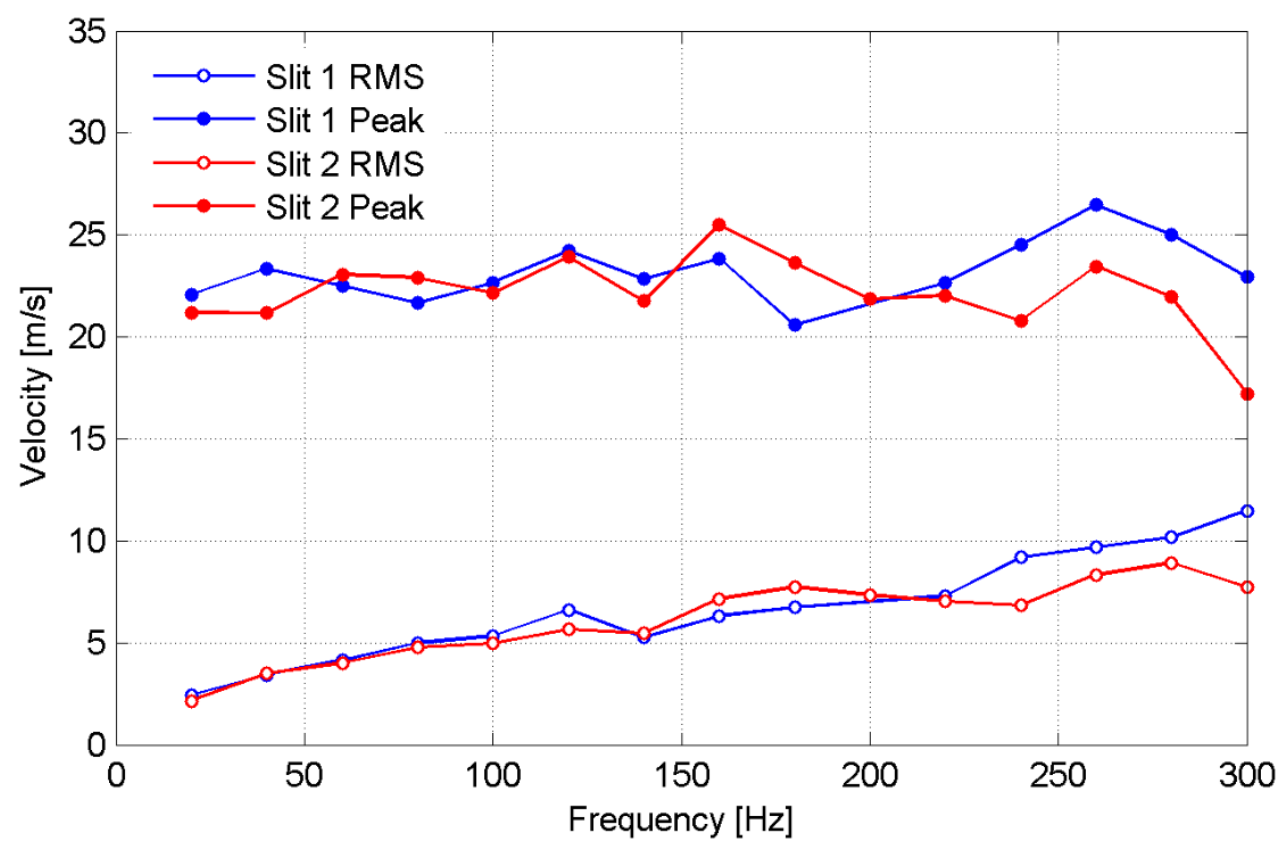

Figure 2.8 Peak and RMS velocities for Slit 1 and 2

The velocity profiles for slits 1 and 2 are not drastically different. This means that velocities of this magnitude may be expected when the actuators are mounted in the model.

\subsection{NACA 0015 Semispan Model}

A major effort in this thesis was the design and manufacture of the NACA 0015 Semispan model. The goal was to keep the design as simple as possible so avoid overcomplicating the manufacturing process. The first step in the design was to figure out the size of the model. The NACA 0015 airfoil was chosen because this was the airfoil used in the previous study. The chord of the model needed to be as small as possible to prevent wind tunnel blockage and to increase $\mathrm{C}_{\mu}$. The problem with decreasing the chord too much was that the actuators and pressure ports could not fit if the chord was too small. After some initial SolidWorks design, a chord of 8 inches was found to be enough to fit the actuators and pressure ports (a detailed SolidWorks drawing will be shown later in this section). Wind tunnel blockage corrections will be used to offset the blockage effect. It was necessary to prove that the synthetic jet actuator could provide sufficient actuation for a wing with an 8-inch chord. For this study, a $\mathrm{C}_{\mu}$ of 0.0008 was needed to match the 
experiment in reference 12. Figure 2.9 contains an estimate of $\mathrm{C}_{\mu}$ for an 8-inch chord with spanwise SJA (synthetic jet actuation) showing that the actuators should be strong enough for this experiment.

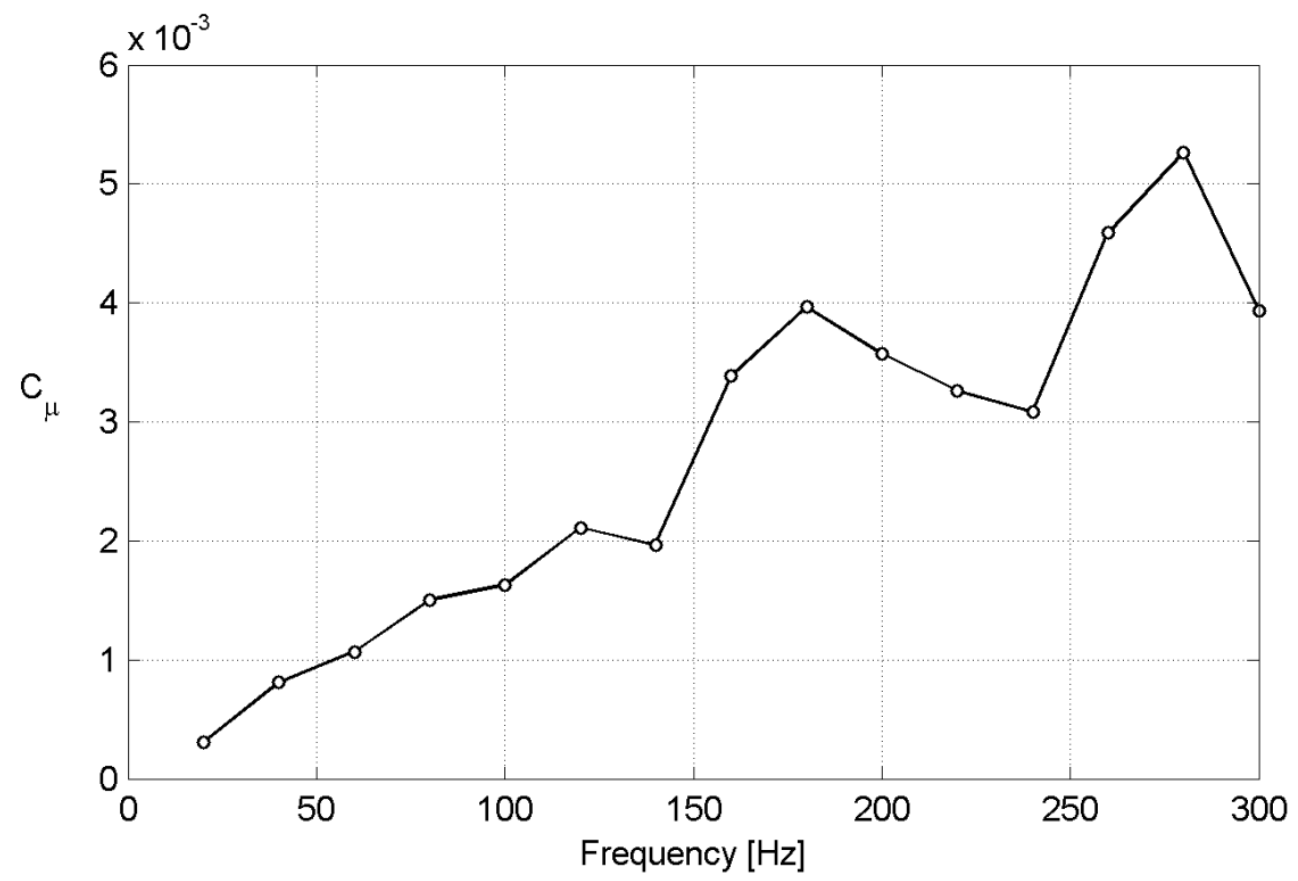

Figure 2.9 $\mathrm{C}_{\mu}$ estimate for spanwise actuation

The momentum coefficient was obtained using the peak velocity data from slit 1 in Figure 2.8. The velocity was corrected using conservation of momentum to represent the actual slit that will be used in the experiment. The estimated momentum coefficient varies from about 0.0003 to 0.0052. After $40 \mathrm{~Hz}$, the estimated value is higher than the largest $\mathrm{C}_{\mu}$ of 0.0008 used in the previous study meaning an 8 -inch chord wing could be used. The span of the model was 23 inches. Although this value is larger than needed for the 2"x2" tunnel, the model was designed so it could be retracted under the tunnel reducing the effective span.

Now that the size of the wing was set, it was necessary to figure out the overall layout of the wing. A key characteristic that made it possible to create a compact model was the idea of turning one synthetic jet actuator disk into two synthetic jet actuators by using the same actuators for 
suction surface and spanwise actuation. A schematic showing this concept is shown in Figure 2.10 .

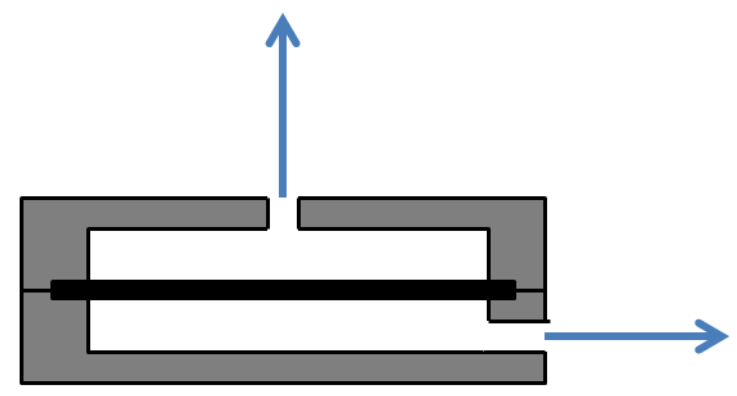

Figure 2.10 Dual synthetic jet concept

Using two cavities, less energy is used for actuation and two jets are produced instead of one. The vertical jet would be used for suction surface actuation and the horizontal jet would be used for spanwise (wing tip) actuation. This concept was expanded by combining several actuators to get one stronger jet since only one jet was needed for spanwise actuation. A schematic of this idea is shown in Figure 2.11.

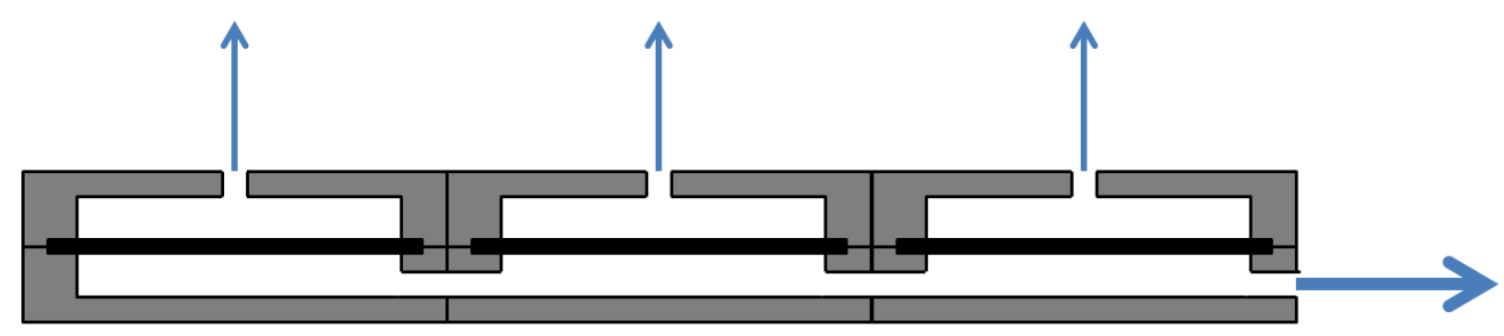

Figure 2.11 Combined actuator concept

The vertical arrows represent suction surface actuation and the horizontal arrow represents spanwise actuation. The layout of the wing was built around this concept. The top half of the wing would be used as half an actuator case providing suction surface actuation and the bottom of the wing would be used as half an actuator case providing spanwise actuation. A wingtip would be built in order to channel the spanwise actuation into a slit that has similar dimensions to the slit used in the previous study. 
The pressure port layout was chosen next. The placement of the pressure ports was effected by a few factors. First, manufacturing constrains made it so that only a certain number of pressure ports could be fit in a given area. In addition, room needed to be made for pressure tubing since it would need to run out of the model and into the RAD3200 System. Second, a study that would be performed by another student needed to sense separation and assess the effect of suction surface actuation along the span. Therefore, pressure ports needed to be spread out in rows along the span and pressure ports were needed near the trailing edge to sense separation. Lastly, pressure ports were needed near the wingtip to see if spanwise actuation had significant effect at the wingtip. The pressure port layout is shown in Figure 2.12
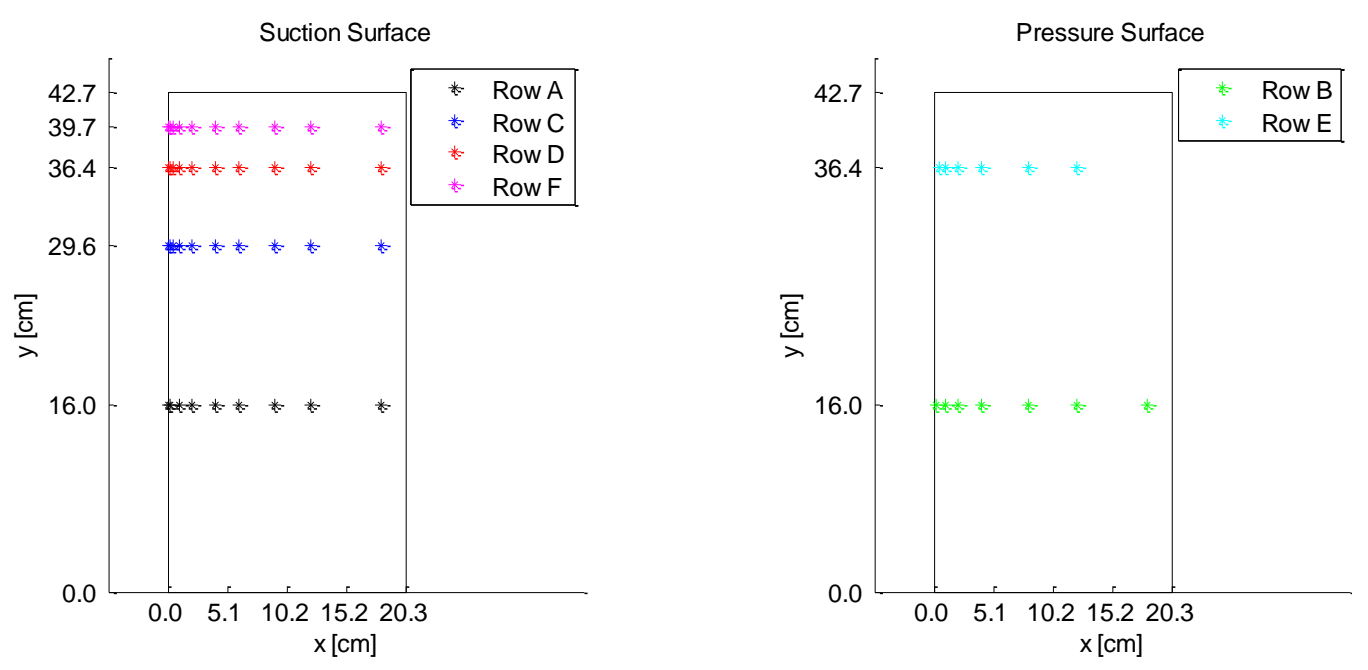

Figure 2.12 Pressure port row locations

The $2 \mathrm{y} / \mathrm{b}$ locations for the rows $\mathrm{A}, \mathrm{C}, \mathrm{D}$, and $\mathrm{F}$ are $37.5 \%, 70.2 \%, 85.1 \%$, and $93.1 \%$ respectively. The $2 \mathrm{y} / \mathrm{b}$ locations for rows $\mathrm{B}$ and $\mathrm{E}$ are 37.5 and 85.1 respectively. More rows were placed on the suction surface than the pressure surface. This is because the other study was more interested about how suction surface actuation affects the pressure distribution on the suction surface than how it affects the distribution on the pressure surface. The pressure port $\mathrm{x} / \mathrm{c}$ locations for rows A, C, D, and F are shown in Table 2.3 and the $\mathrm{x} / \mathrm{c}$ locations for rows $\mathrm{B}$ and $\mathrm{E}$ are shown in Table 2.4. 
Table 2.3 Top pressure port locations

\begin{tabular}{|c|c|}
\hline Port \# & $x / c[\%]$ \\
\hline 1 & 0.2 \\
\hline 2 & 0.5 \\
\hline 3 & 1.3 \\
\hline 4 & 2.1 \\
\hline 5 & 4.7 \\
\hline 6 & 10.0 \\
\hline 7 & 20.0 \\
\hline 8 & 30.0 \\
\hline 9 & 45.0 \\
\hline 10 & 60.0 \\
\hline 11 & 90.0 \\
\hline
\end{tabular}

Table 2.4 Bottom pressure port locations

\begin{tabular}{|c|c|}
\hline Port \# & $x / c[\%]$ \\
\hline 1 & 1.0 \\
\hline 2 & 2.0 \\
\hline 3 & 5.2 \\
\hline 4 & 10.0 \\
\hline 5 & 20.0 \\
\hline 6 & 40.0 \\
\hline 7 & 60.0 \\
\hline 8 & 90.0 \\
\hline
\end{tabular}

The pressure ports were clustered near the front of the wing to capture the large pressure gradients at the leading edge. Pressure port 4 in row A was non-functional due to damage caused during manufacturing and will not be used in this experiment.

The next step in the design process was to choose the materials for each part of the model. The wing material needed to have several qualities. First, it had to be machinable. The machining process would take many hours and require the use of many small specialty end mills. If the material were something tough or abrasive, end mills would have a very short lifecycle. A machining time estimation by CAMWorks showed that the setup will take at least 8 hours to machine. For machining operations this long, the machine is generally run overnight. If an end mill breaks during this process, the machine would continue running and it would affect the next milling feature most likely breaking that end mill too. This would lead to many broken end mills and an unfinished part. This whole problem could be avoided by choosing an easily machinable material. The next consideration was stiffness. A stiff material was needed so it does not deflect under aerodynamic forces and break the stiff actuators. A material was also needed that did not conduct electricity because $180 \mathrm{Vpp}$ would be supplied to each actuator providing a current of up to $200 \mathrm{~mA}$. If the wing was conductive and the model was touched while the actuators were one, 
the user could be shocked by the high voltage and current. With all of these factors taken into hand, materials considered were wood, plastic, and ceramic. Wood was discarded because inconsistencies in grain can create gaps creating leaks in the actuators, also wood clogs coolant filters for the milling machines. Machinable ceramic (MACOR) was eliminated because even though it is labeled as "machinable" it is very abrasive and causes tools to wear very quickly. This was not acceptable for an 8 hour operation. It is also very brittle and a light bump can shatter it. Plastic seemed like the best choice. Several plastics were considered but polycarbonate and acrylic were favorable because they are clear. It would be easy to assess the condition of the model (e.g. actuator damage) with the structure being clear. Polycarbonate is more machinable and much less brittle but is it is also less stiff. It was thought that the polycarbonate would be too flexible and break the actuators under aerodynamic forces. Therefore, acrylic was chosen for the wing structure. The wing spars were initially of aluminum construction to reduce the weight of the assembly but after testing, it was realized that aluminum is too flexible and a steel spar was made.

Now that the size, material, overall layout, and pressure port locations are set, the wing was designed in SolidWorks so it could be manufactured. The top half of the wing is shown in Figure 2.13 


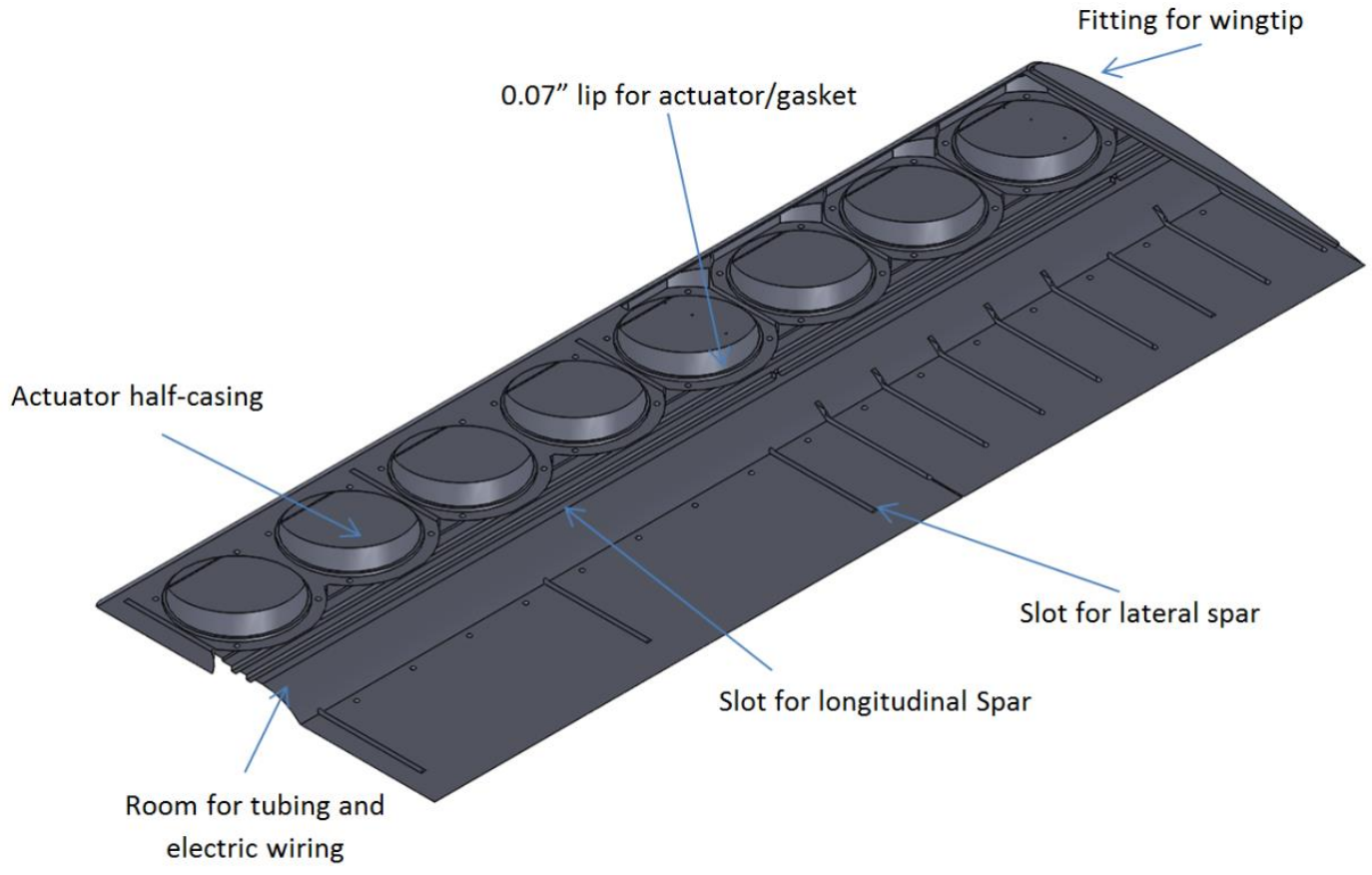

Figure 2.13 Top half of the wing showing internal details

The top half of the wing was designed so it could be built out of one piece of material using three mill part setups. It will have lateral and longitudinal steel spars to prevent it from snapping in half in the wind tunnel, eight casings for actuators with room between each case for ducting, and a male fitting on which the wingtip will be placed. Near the tip of the wing, the leading edge has material removed to make room for pressure ports. Most of the pressure port holes were designed to be drilled on the $\mathrm{CNC}$ although near the leading edge this was impossible due to the high curvature. Near the leading edge 0.01 " holes at a $0.005 "$ depth were added. These would serve as markings to where the leading edge pressure ports are to be and drilled by hand. A 0.01 " endmill would be used to make these markings on the CNC. Screw holes were added on both sides of the longitudinal spar. On the leading edge side the screw held a dual purpose of holding the two wing halves together as well as clamping the actuators together. A $2.130 \times 0.039$ " slit was cut at a 30 degree angle from the surface of the wing in each actuator at $10 \%$ chord for another study. Choosing to cut the slit at a $1 \mathrm{~mm}(0.039$ ") width and a 30 degree angle involved careful consideration. The student doing the separation control study needed a slit no greater than 0.039 " 
cut at as small of an angle to the surface as possible so the jet is blowing downstream. It was necessary to find an endmill that could make this cut. From previous experience machining the synthetic jet, the thinnest possible actuator cavity ceiling thickness was 0.04 ". Any thinner and the acrylic would warp from machining and could even crack. Therefore, if cutting perpendicular to the surface $(\Theta=90$ degrees, where $\Theta$ is the angle of the cutter relative to the surface), the flute length of the endmill would need to be 0.04 ". As the slit angle decreases the flute length needed to make the cut increases with $1 / \cos (\Theta)$. Since endmills have a direct relationship between max flute length that can be manufactured per endmill and endmill diamter, a larger endmill would make it easier to cut at a smaller angle. Therefore, the maximum allowed slit width of $1 \mathrm{~mm}$ was chosen. The next issue was choosing the angle to cut. One constraint was that it is harder to get a $\mathrm{CNC}$ mill chuck close enough to the surface of the wing as you decrease the slit angle because you have to fixture the model at an angle to make the cut. Another constraint is that the maximum flute length for readily available $1 \mathrm{~mm}$ endmills is $4 \mathrm{~mm}$. After considering these geometric constraints, it was found that a 30 degree slit could be cut.

The next part to be designed was the bottom surface of the wing, shown in Figure 2.14. The pressure surface is shown in thise view (the internal sturctre is not shown because it is almost exactly the same as in the top half shown in Figure 2.13). 


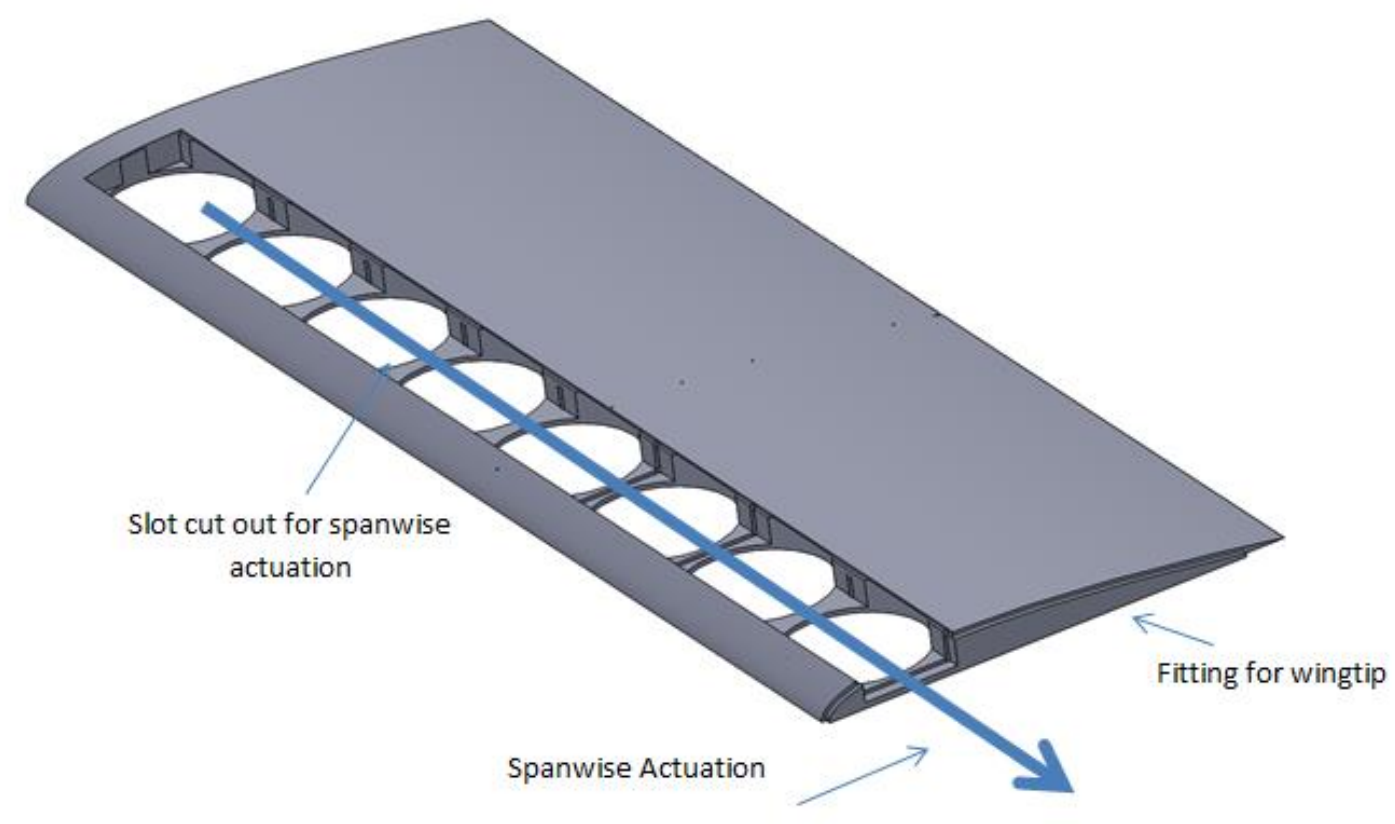

Figure 2.14 Bottom half of the wing showing pressure surface.

A slot was cut out to connect all of the actuator cavities similar to the way shown in Figure 2.11. The heavy blue arrow shows the direction of flow. A thin 0.04 " cover was designed to cover this slot to make a single complete cavity. The cover is shown in Figure 2.15. The top and bottom wing halves are designed so they could be screwed together to form the wing and serve as the actuator cavities. Wingtip fittings on the top and bottom surface match up so that a male fitting is made for the wingtip. The wingtip will be discussed in the next section. The two halves of the wing will also clamp down on the longitudinal and lateral spars for structural support. The longitudinal spar, shown in Figure 2.16, will stick out the side opposite of the wingtip and will serve as a way to hold the wing. The spar has 0.1 " holes drilled into it in which the lateral spars are placed. 


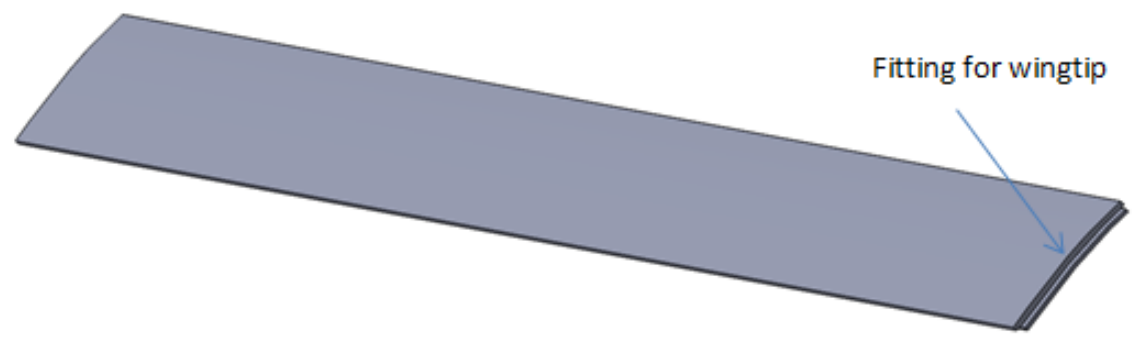

Figure 2.15 Pressure surface actuator cover

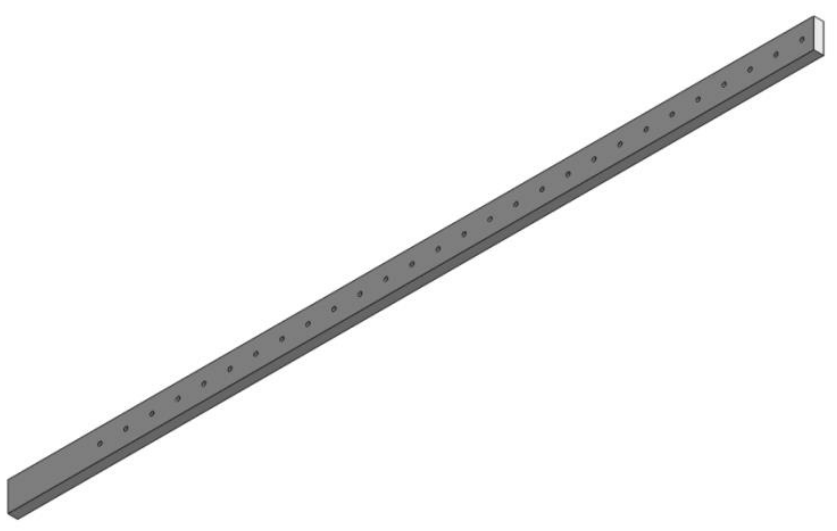

Figure 2.16 Wing longitudinal spar

The lateral spars are steel rods with a 0.1 " diameter and prevent the wing from shearing due to the moment caused by aerodynamic loads. They also prevent the wing from sliding down the longitudinal spar. The holes were placed every 0.667 " to allow to model to be raised by 0.667 " inches if necessary. $0.667^{\prime \prime}$ was chosen because the lateral spars slots could only be placed between the actuators and 0.667 " is a quarter the distance between actuators, allowing for an even hole spacing.

The last part of the wing design was the wingtip. The wingtip has to fit on the fitting created by the both halves of the wing and the cover. The wingtip also needs internal ducting to direct the flow from spanwise actuation to the slit. Figure 2.17 shows the wingtip as if looking into the yaxis of the wing. 


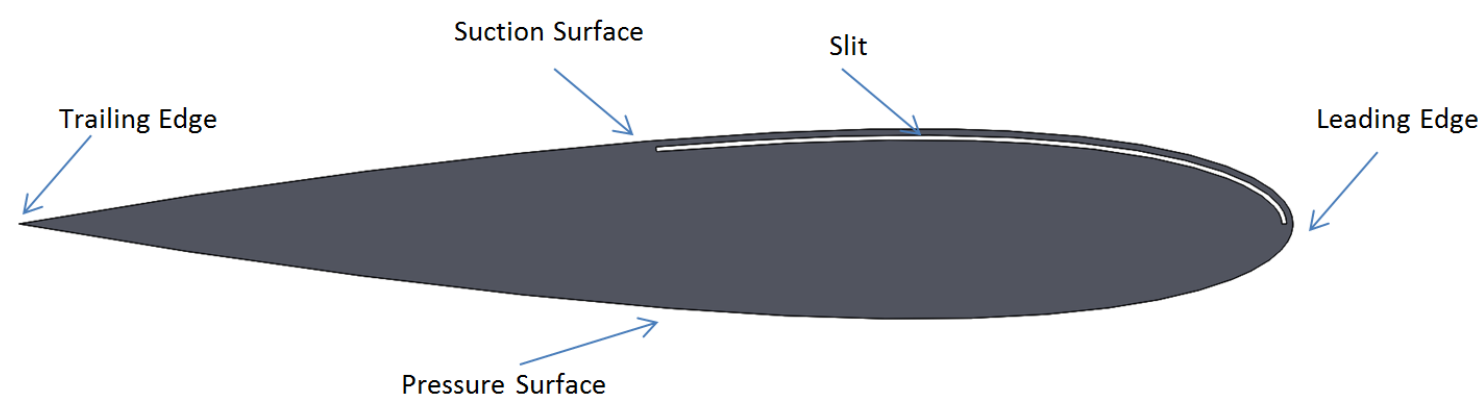

Figure 2.17 Wingtip

The slit location is based on a previous study ${ }^{12}$. In the previous study, several slit locations were studied although the most diffused vortex was seen when the slit was placed near the suction surface with the jet emanating spanwise. Therefore, this slit location was chosen for the current study. The slit used in the current study is 0.04 " wide and 4.128 " long. The width was chosen to be as small as possible to allow for room for machining error. Although the $\mathrm{CNC}$ is very accurate and can machine something to within 0.0001 ", other error sources could be present. These include human error in fixturing, worn out end mills leading to smaller than listed diameters, material warping, and many others. These error sources could not be precisely quantified but it is estimated form previous machining experience that this error usually does not exceed 0.01 ". if care is taken. Since the wingtip is manufactured from two halves, the maximum error could be 0.02 ". so the actual slit width could vary from 0.01 to 0.05 ". This range was considered acceptable for the current study. The slit length for the current slit was made proportional to the slit length used in the previous study. Figure 2.18 shows a SolidWorks model of the wingtip. The material was made to be transparent to show the internal duct. 


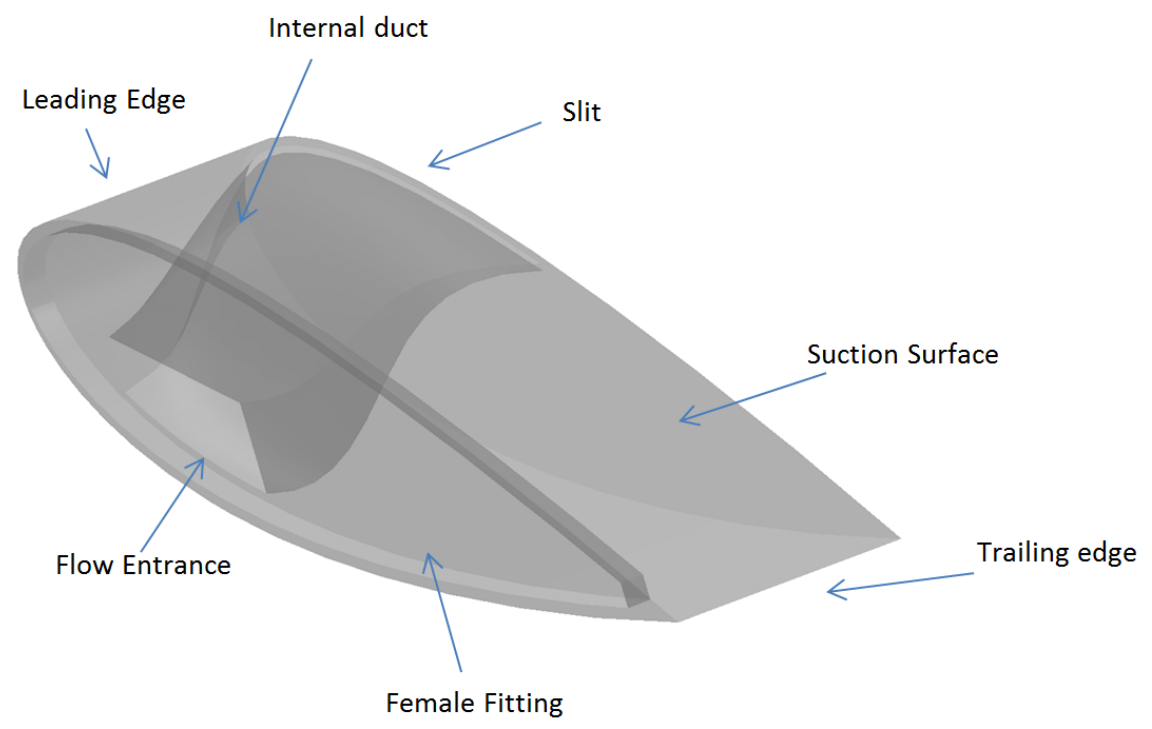

Figure 2.18 Wingtip

The flow from the bottom wing half travels into the flow entrance and is channeled through the internal duct. The flow exits the internal duct from the slit providing spanwise actuation out of the wingtip. This wingtip design is impossible to manufacture using a 3 axis $\mathrm{CNC}$ milling machine in the single-piece configuration shown. This was considered and the wingtip was therefore designed so it could be built in two halves which can be glued together to produce the configuration shown in Figure 2.18. These halves are shown in Figure 2.19
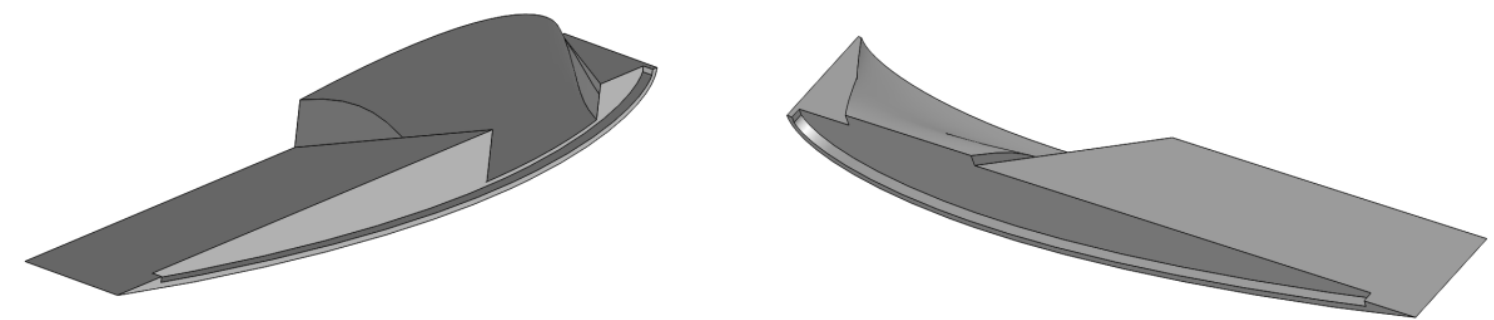

Figure 2.19 Wingtip halves

Now that each piece of the wing was designed and modeled in SolidWorks, the wing could be manufactured. 
The first step of the machining process was to create the g-code code for the CNC machines. CAMWorks is a software that makes this a seamless process. Using CAMWorks, each feature on each of the solid models was converted into a milling operation. These milling operations consist of toolpaths that the user defines and customizes by changing various operation parameters. The higher-level parameters are, milling speeds and feeds, end mill diameters, and depth of cuts but many more exist. CAMWorks converts all of this information into g-code, which is that language that the CNC reads. An example of one of these toolpaths is shown in Figure 2.20. The blue line is the path the center of the end mill will follow during machining. The toolpaths were simulated once all of the operations were finalized as shown in Figure 2.21. Simulating the toolpath serves as a safety check since the toolpath is simulated exactly as the CNC will machine the feature showing the end mill and the original stock. The simulation shows if the $\mathrm{CNC}$ will create unwanted cuts or leave uncut material in certain places. The screenshot shown in Figure 2.21 shows the machining of the lip in which the gasket and actuator will sit. Each half of the wing had about 60 different operations.

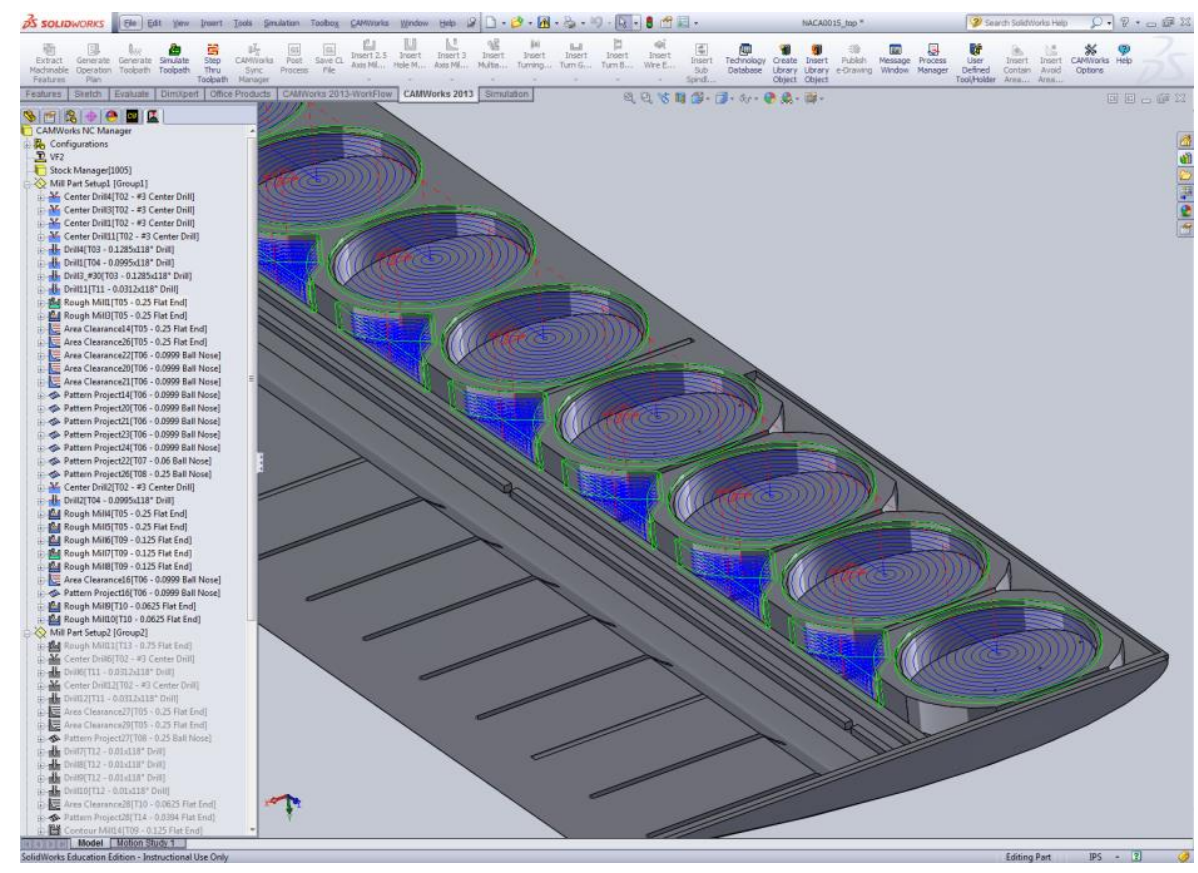

Figure 2.20 Screenshot showing selected toolpaths 


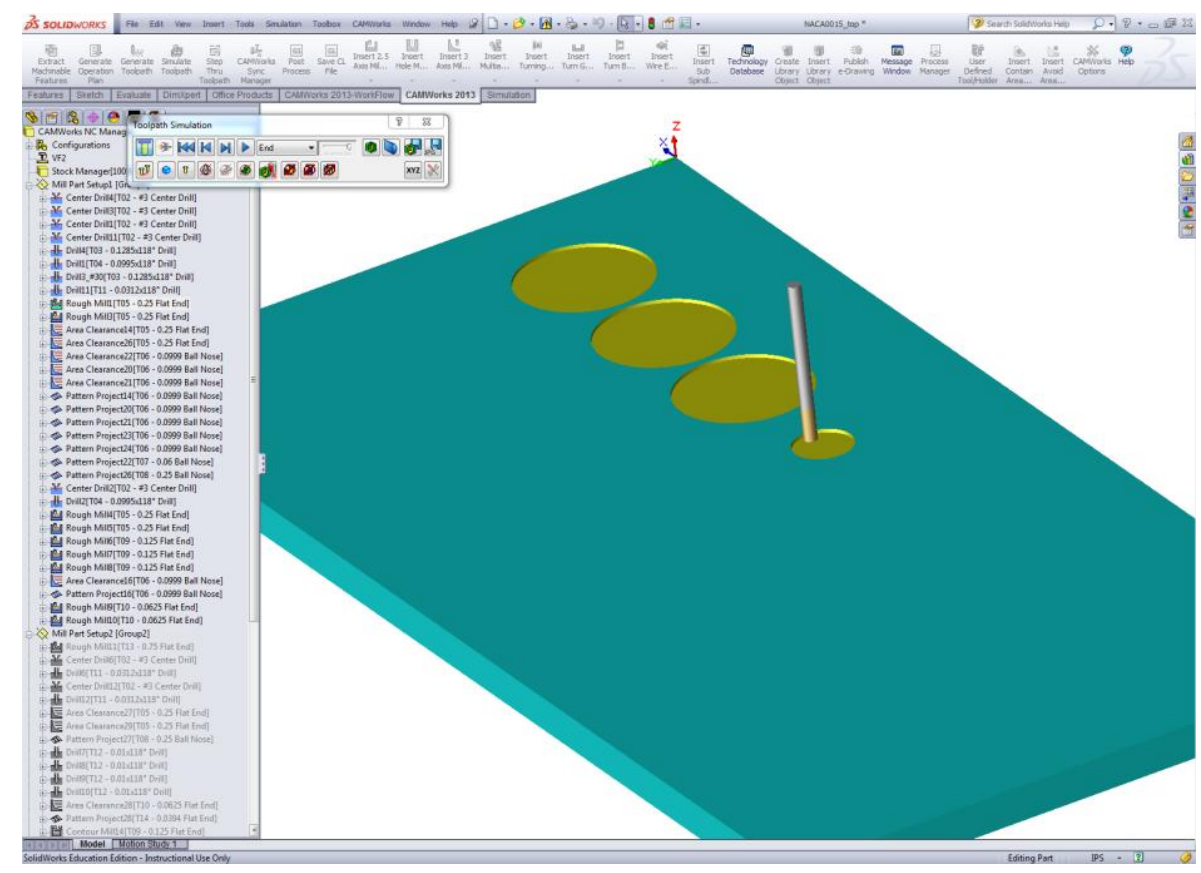

Figure 2.21 Screenshot showing toolpath simulation

Once all of the g-code was written the raw stock and CNC could be prepared for machining. The first piece that was machined was the wing top half. A $12 \times 24 \times 1$ inch piece of acrylic was used as the raw stock. Generally, stock needs to be made into a perfectly square shape so it could be fixtured precisely. This was very difficult because plastic stock of this size comes slightly warped when purchased, does not have any relatively flat surfaces, is not square, and has internal stresses. Whenever a surface was milled off to make the stock square, the internal stress distribution changes and the material warps again. Since all of the sides need to be flattened, squared, and the material kept warping after each cut, the traditional squaring methods could not be used. Two ideas were thought of to make it possible to square the material. First idea was to anneal the acrylic to remove the internal stresses. This could be done by heating the acrylic to $180^{\circ} \mathrm{F}$ over the course of two hours, holding it in the oven at $180^{\circ} \mathrm{F}$ degrees for 30 minutes per $1 / 4$ inch thickness and cooling it down at a rate of $50^{\circ} \mathrm{F}$ per hour. The other method would be to sand one surface on a flat table until it would stop warping. This method does not remove internal stresses but it makes the stock flat. The stock still had to be squared on the mill but the amount of material that needed to be removed was much less so the internal stress distribution didn't change 
much and the stock warping was insignificant. The sanding method was chosen because it was much faster than the oven method. The sanding was done by taping sand paper to a precision granite table and running the surface of the acrylic across it.

Once the stock was square, the material needed to be fixed to the VF-3. Because the stock is large, a vice could not be used. The acrylic was placed directly on the table then clamped down at each corner of the stock using toe clamps. The tools were inserted into the VF-3 and the wireless tool setter was used to measure the tool offsets. A dial indicator was used to make sure one edge of the stock was parallel with the x-axis. The wireless probe was used to measure the stock at the top and at a corner to get the part offsets. Once this was done, the coolant was turned on and the machine cycle was started. A picture of the wing being machined is shown in Figure 2.22.

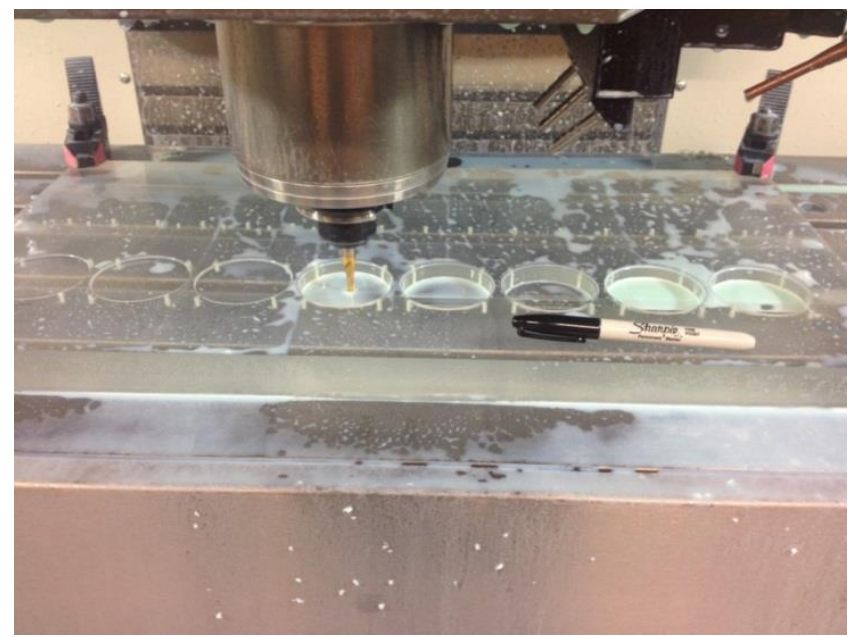

Figure 2.22 Wing top half being machined.

Once the cycle was complete, the stock was flipped over to cut the other side. For this part setup, a sheet of polycarbonate was used to raise the stock to prevent the end mill from hitting the table. During the machining process, the wing was bowing up due to the internal stresses. To combat this, sand bags were placed on the wing in different places. Once the cutter moved close to the sandbags, the cycle was paused and the sand bags were moved over. For the third part setup, the wing was fixtured at an angle so the slits could be cut. A similar procedure was followed although only two part setups were needed since slits did not need to be cut at 30 
degrees for the pressure surface. Figure 2.23 shows both halves of the wing after machining was completed. Figure 2.23a shows the suction surface of the top half, and Figure 2.23b shows the pressure surface of the bottom half. Both surfaces were sanded starting at 60 grit and working up to a 600 grit paper to get the surfaces smooth. The acrylic was then treated with methylene chloride, which is an acrylic solvent. Methylene chloride slightly melts the surface getting rid of any leftover scratches from the sand paper giving a mirror like finish.

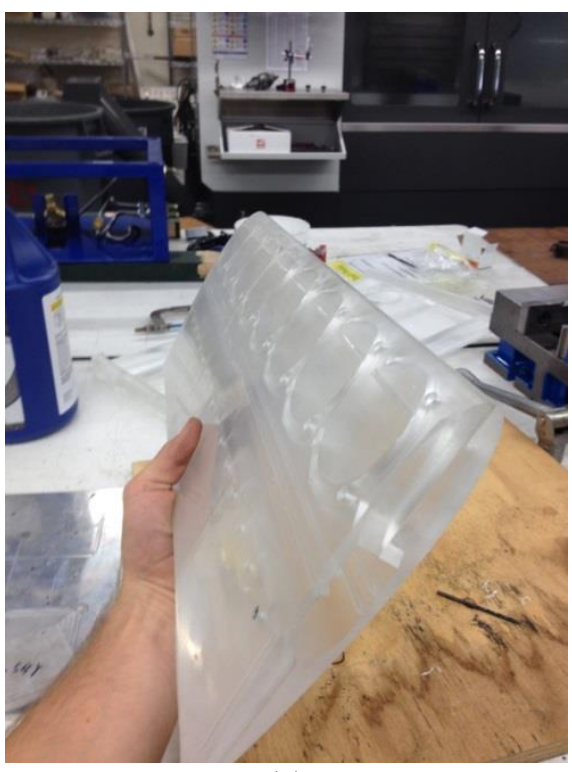

(a)

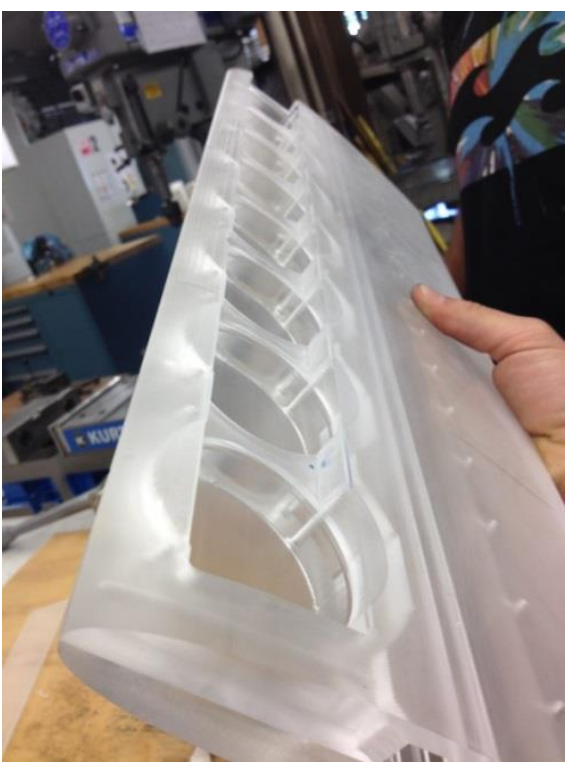

(b)

Figure 2.23 Both halves of the wing

The next parts that were machined were the wing spar and the pressure surface cover. These parts were also machined on the Vf-3 due to their size. The fixturing was similar to how the wing was fixtured, as a vice could not be used.

The wing tip was manufactured next. Due to its small size, the Minimill was used. The wingtip was machined in two halves each consisting of 3 part setups. Each half of the wingtip was fixtured in a vise. Besides this, the machining procedure was just as it was on the VF-3. The finished wingtip is shown in Figure 2.24. 


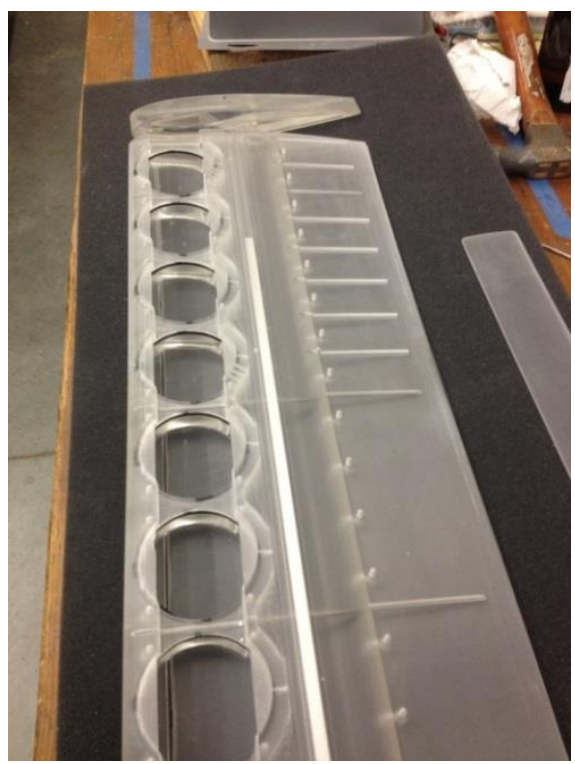

(a)

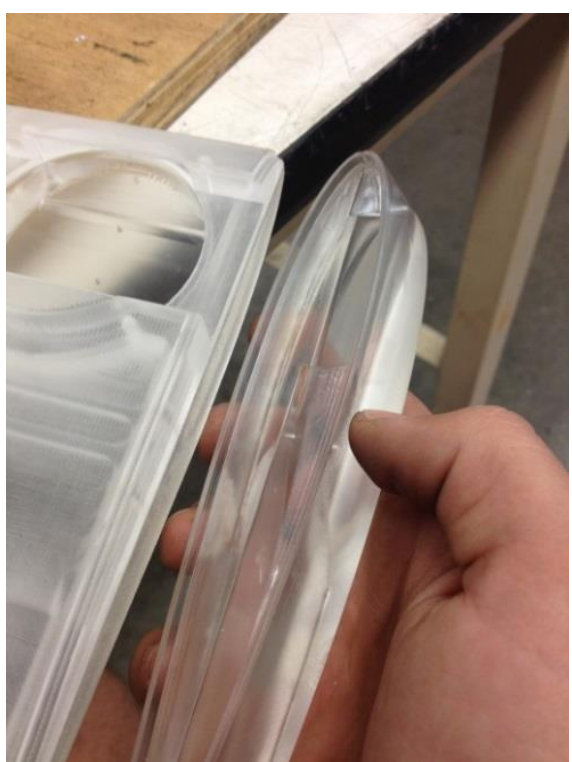

(b)

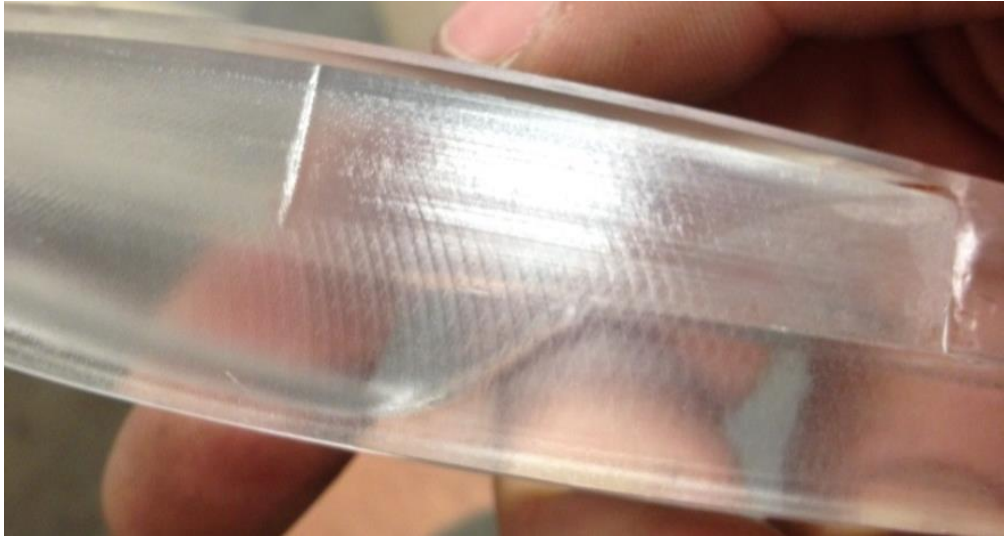

(c)

Figure 2.24 Both halves of wingtip

Figure 2.24a shows the wingtip relative to the wing. The spars and wing cover can also be seen in this picture. Figure 2.24b shows the fitting and Figure 2.24c shows the slit.

Now that each component of the wing was manufactured, the wing had to be assembled. This included inserting the actuators and pressure ports. This involved several steps. First, leads were soldered to the Bimorph disks. This was done using miniature high flex wire and a standard flux-solder kit. The gaskets were then cut. These were made the same as they were for the synthetic jet actuator in Section 2.2. The actuator disks and gaskets are shown in Figure 2.25. 
Figure 2.25a shows the leads attached to the disks and Figure 2.25b shows the disks sitting in the wing. The gaskets can also be seen on the outer edges of the actuators.

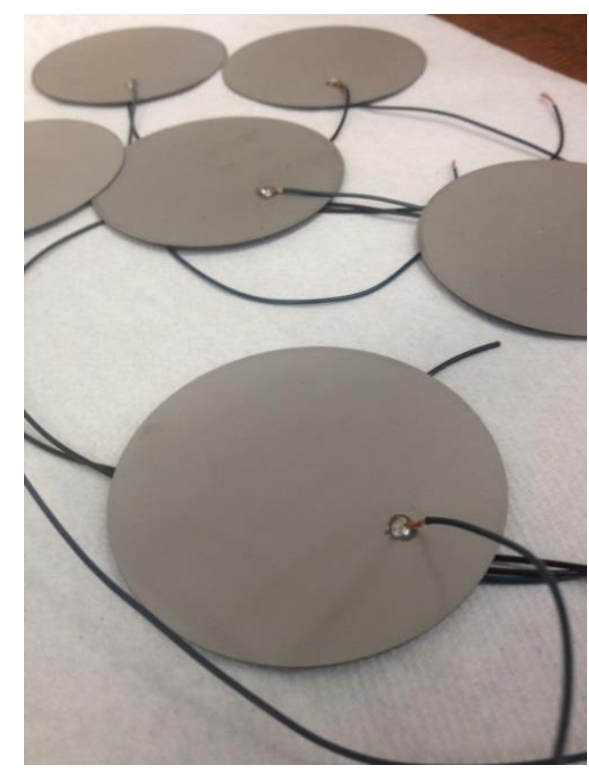

(a)

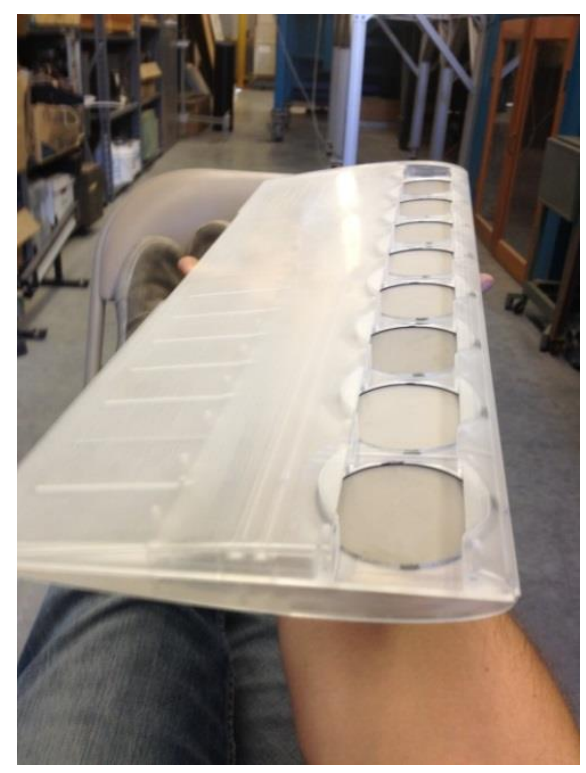

(b)

Figure 2.25 Bimorph disks and gaskets

The next step was inserting the pressure ports. Most of the pressure port holes were already drilled using the VF-3. The leading edge pressure ports needed to be hand drilled at the 0.01 " markings created with the VF-3. Next, 0.042" OD 0.027" ID steel tubing was cut, bent, and inserted into the holes to serve as fittings. Tygon tubing was attached at the end of each fitting and was fed out the model to be later attached to the RAD3200 System. Some of the pressure ports were inside the actuator cavities. Holes had to be manually drilled through the sides of the cavities for the tubes to be fed out. The pressure ports are shown in Figure 2.26. Figure 2.26a shows the holes that were drilled for the steel fittings. This figure also shows the slit and the screw holes. The holes near the tip were staggered to make it easier to insert the fittings. Figure 2.26b shows the fittings, the tubing, and the holes drilled into the side of the actuator cavity for the tubing. With close examination, holes that were drilled for the actuator leads can also be seen. 


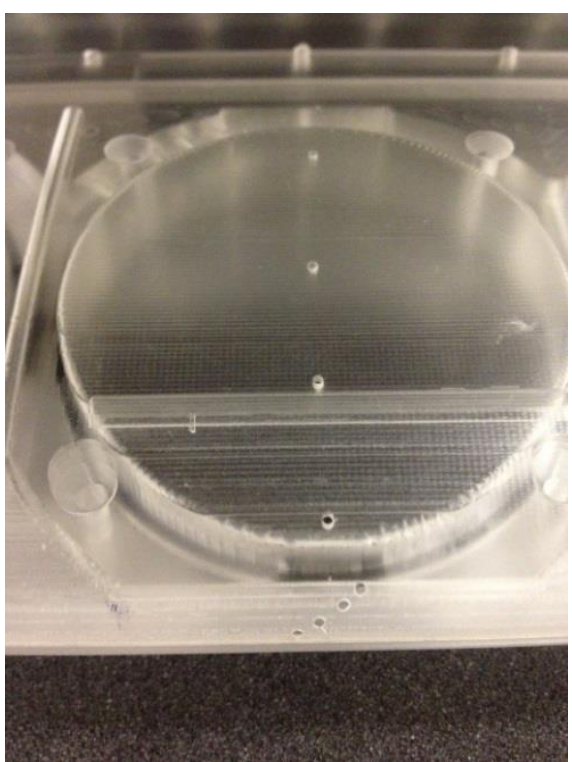

(a)

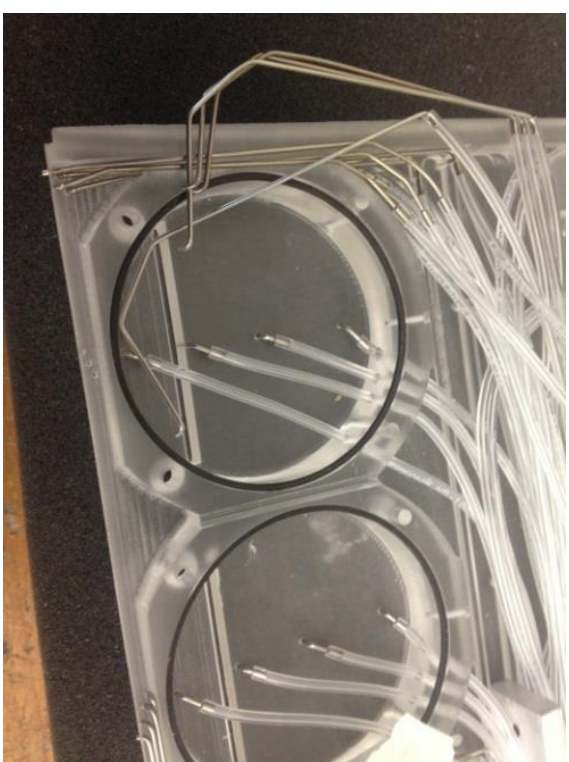

(b)

Figure 2.26 Pressure ports

\subsubsection{Model Accuracy}

When machining a part, it is impossible to get it $100 \%$ exact. Great care was taken to machine this wing exactly as designed in SolidWorks but several factors affected this. First, the stress distribution in the acrylic changed after every pass of the end mill. Even though the wing was fixtured all along the edges, the center could bow up or twist. When machining the suction and pressure surfaces, the wing bowed up slightly. This was combatted by placing sand bags on the wing to hold it down although the bowing could not be eliminated completely. Because of this, the chord is about 0.2 " shorter in the middle of the half-span wing. It is also slightly less thick than a NACA 0015 of 8 inch chord near the middle. The pressure port holes placed by the machine are very accurate compared to a perfect NACA 0015 wing but since there was bowing, the effective pressure port locations are slightly off near the middle of the wing. This could not be avoided. Also, the pressure ports at the front of the wing were done by hand. The locations for the holes were marked with a 0.01 " end mill but since the holes were drilled by hand there is human error. Another error in the model is in the suction surface slits. These were by far the most difficult machining procedure in the model. Some of the slits are slightly larger than $1 \mathrm{~mm}$. This is 
because the 0.04 " thick wing walls vibrated a lot while the slits were being machined and caused more material to be taken away.

\subsection{Load Cell Support Structure}

It was necessary to measure the lift and drag on the wing. This experiment was originally designed for the $3 \times 4$ Aerospace Wind Tunnel which does not have a good way to measure the loads on a semispan model. Because of this, a device was built for this purpose. The idea was to use an Omega LCFD-1KG load cell. If the wing could be made to translate in one direction due to load on the wing, then a load cell could be used to measure the load. The angle of attack also needed to be adjustable. The concept that was developed is shown in Figure 2.27.

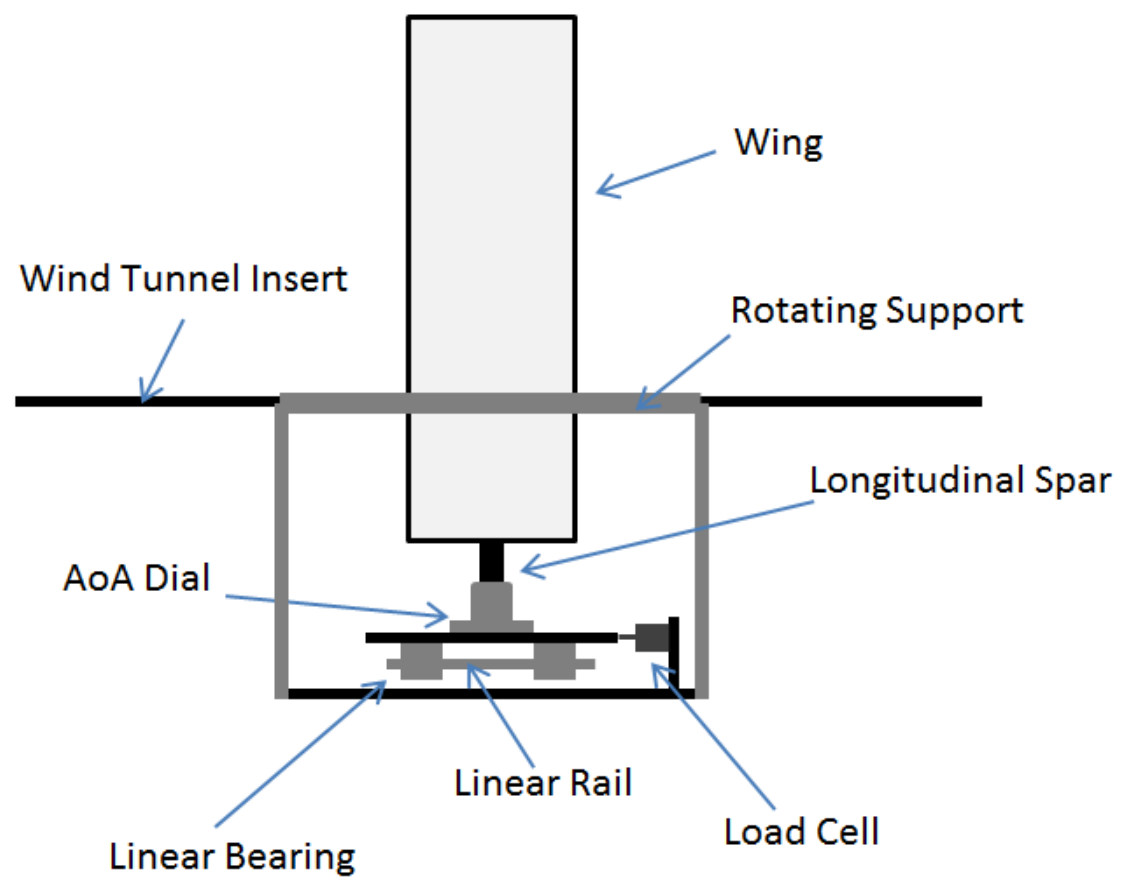

Figure 2.27 Load cell support structure concept

The wing sits on plate containing a dial that rotates to change the angle of attack and the spar is inserted to the center of the dial. Three linear bearings are attached to the bottom of the plate. These restrict the motion of the wing to one axis and prevent rotation. The original idea was 
to use 4 bearings but it was realized that three bearings can be used with the same effect if placed in a triangular pattern. Under aerodynamic loads, the plate presses into the load cell and the load cell measures the load. The rotating support was designed so the whole assembly can rotate change from measuring lift to measuring drag. This switch can be done by rotating the assembly 90 degrees. The apparatus would be inserted into the wind tunnel by removing the bottom of the test section and replacing it with a wooden insert to which all of the load cell and wing support structure is mounted. Every component was built except the bearings and rails, which were purchased. Figure 2.28 shows each of the components as they were designed in SolidWorks.

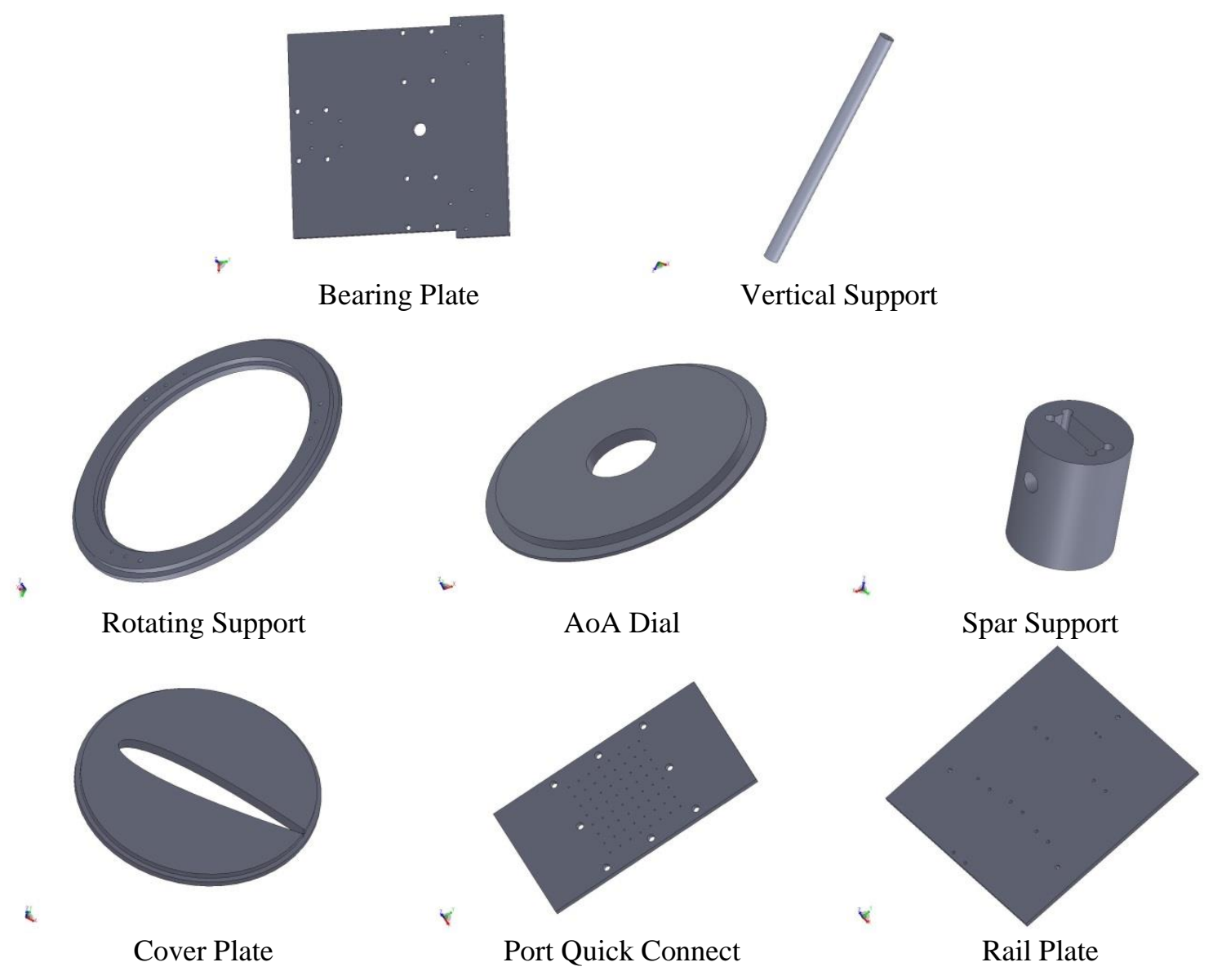

Figure 2.28 Support structure components in SolidWorks 
The linear rail will be screwed to the rail plate. The bearings will be put on the linear rails and screwed to the bearing plate. The spar support will be press fit into the AoA (angle of attack) dial. The AoA dial will sit on top of the bearing plate to be held down by clamps. The cover plate sits in the rotating support and the model sticks out of it. The vertical supports are used to attach the rail plate to the rotating support. The test section pressure was lower than ambient, therefore an airtight case needed to be built so air does not leak through the cover plate into the tunnel and distort data. The port quick connect was used to get the pressure ports through the wall of the case. All of the SolidWorks components were manufactured on the VF-3 except the vertical supports, which were cut to length, drilled, and tapped with the TL-1. Figure 2.29 shows the setup after manufacturing and assembly.

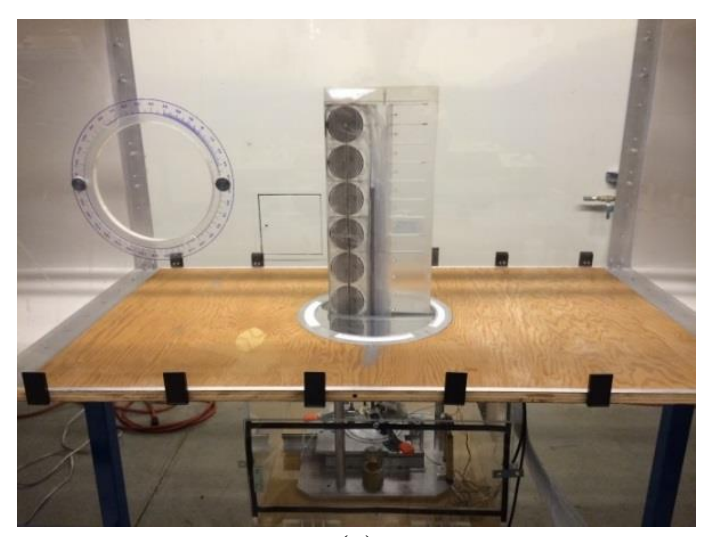

(a)

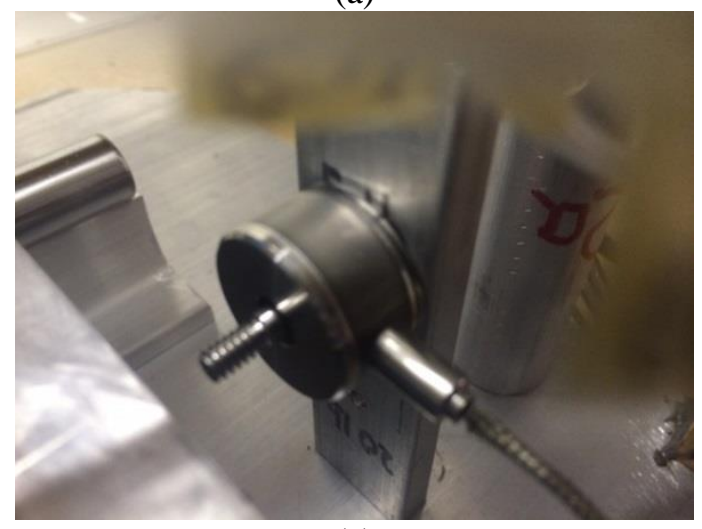

(c)

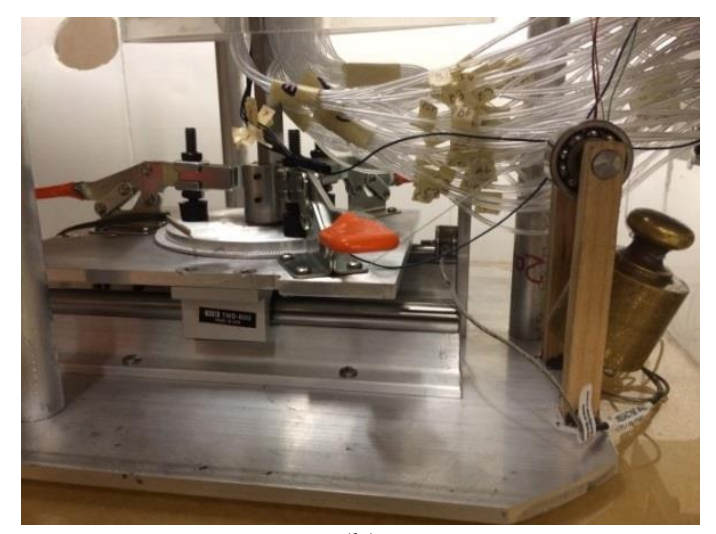

(b)

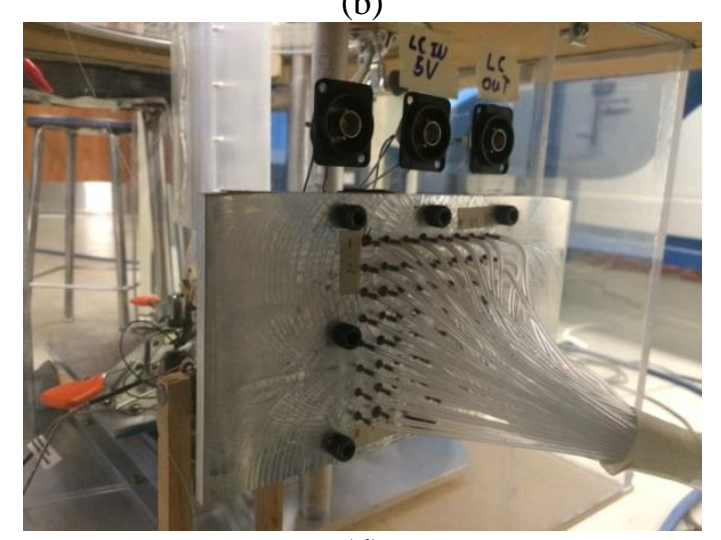

(d)

Figure 2.29 Photos of support structure

Figure 2.29a shows the entire setup. The wing can be seen going thought the cover plate. The wooden insert had a 12 inch hole in which the Rotating Support sits. This hole was made 
using a router and a circular guide. Black clamps can also be seen attached to the insert. These are used to clamp the insert into the tunnel. Figure $2.29 \mathrm{~b}$ shows an up-close picture of the supporting components. The AoA dial, bearing/rail system, and load cell can all be seen. Clamps were used to hold down the AoA dial and fix the angle of attack. A weight was hung to pull the system into the load cell, providing a preload. This prevents small vibrations from slamming the bearing plate into the load cell. Figure 2.29c shows the Omega LCFD-1KG load cell as it is mounted. Under aerodynamic loads, the bearing plate presses into the load cell. Figure $2.29 \mathrm{~d}$ shows the coupling though the acrylic case. BNC connectors were used to couple the load cell and actuator electric wires though the case. The port quick connect was used to couple the pressure ports though the case. 


\section{Experimental Apparatus}

The experimental apparatus was setup in the Cal Poly Mechanical Engineering Fluids Lab. The apparatus was made to be portable, as other labs needed to use the tunnel. A schematic of the experimental apparatus is shown in Figure 3.1 and can be referred to throughout Section 3.

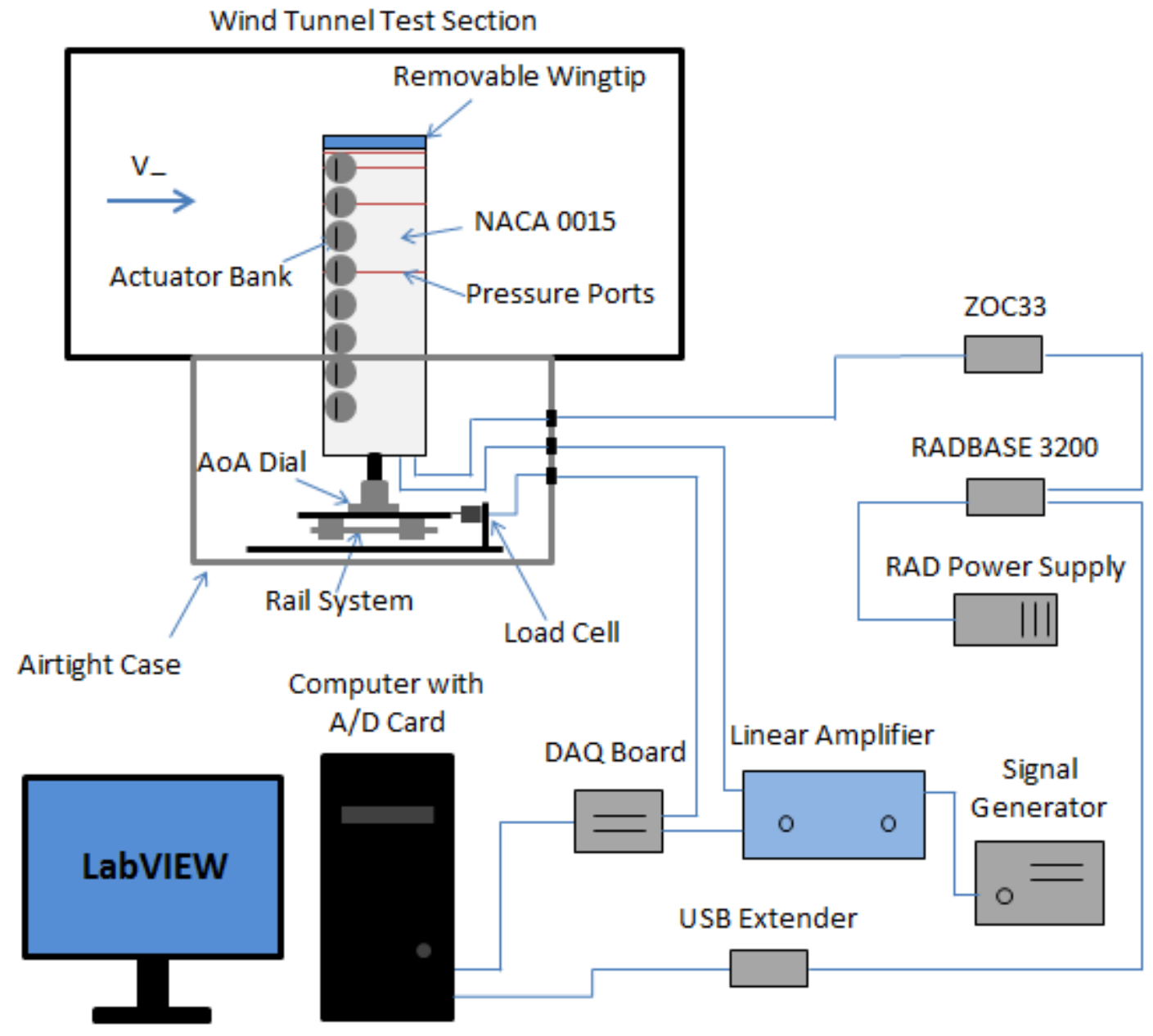

Figure 3.1 Schematic of Experimental Apparatus

\subsection{LabVIEW}

This experiment requires that several devices work simultaneously with the actuators and take data. The actuator bank, the RAD3200 System, and load cell all needed to be controlled. Originally, the plan was to run each of these devices separately using different programs. This would be very time consuming so a LabVIEW virtual instrument (VI) was written to control all 
of these devices at once. A screenshot of the VI is shown in Figure 3.2 with the block diagram in Appendix C. Pieces of source code from the Scanivalve LabVIEW Software Development Kit version 1.3 were used in the making of this VI.

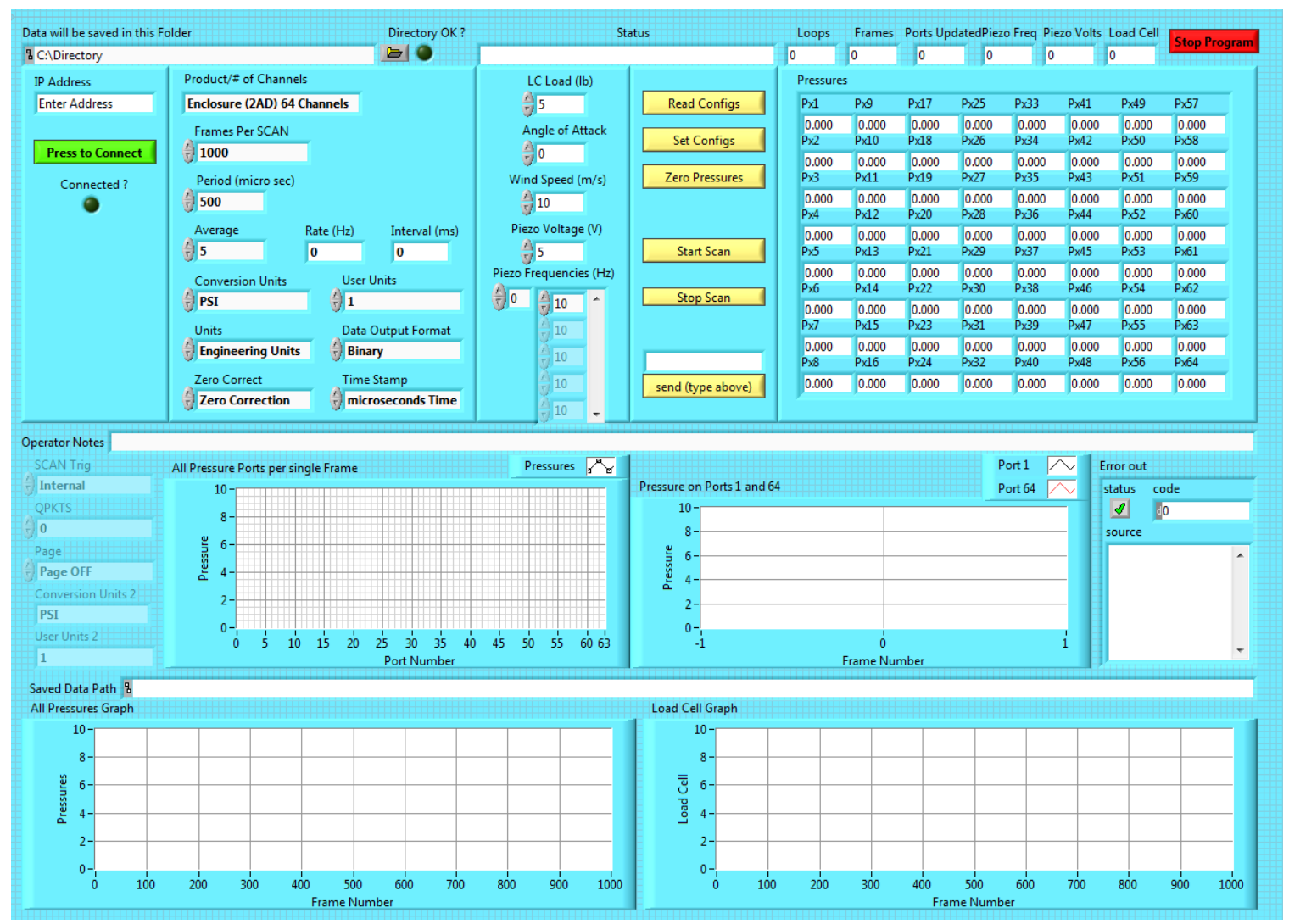

Figure 3.2 LabVIEW Master.VI Screenshot

The VI saves text files, which can be read by Excel, to a directory specified by the user. The test conditions, which include speed, angle of attack, and load cell size can be inputted into the VI and are saved in the header of the text file. The user connects to the RADBASE 3200 and sends commands exactly as the standard Scanivalve software does. All the major parameters commanded to the RADBASE 3200 are saved in the data file. The user then inputs the frequency and amplitude of the square wave to be sent to the amplifier. Once the scan starts, the VI sends a continuous signal to the amplifier and starts taking frames of pressure data. During each frame, a set number of load cell acquisitions are taken, averaged, and plotted in the bottom right of the VI. All of this data is written to the data file at the end of the scan. 
This VI has several capabilities that make testing more convenient. An instantaneous and time continuous graph of pressures was added as a capability that is not available in the standard Scanivalve software. This added capability was helpful for seeing real time pressure port data as well any unsteadiness in the flow around the wing. This VI can also automate a large portion of the testing process. The user can input an array of frequencies or amplitudes. The VI will then conduct a scan at each frequency or amplitude specified. In addition, a quick sweep of frequency or amplitude can be taken. A starting and ending value is entered and a scan is initiated. LabVIEW then takes the first frame at the starting value incrementally increasing the value for each frame until the last frame at the final value is reached. This allows for a quick sweep of the design space to quickly search for any interesting phenomena.

\subsection{Wind Tunnel}

The current experiment was performed in wind tunnel Model 406 built by Engineering Laboratory Design, Inc., which is contained inside the Cal Poly Mechanical Engineering Fluids Lab. This tunnel, shown in Figure 3.3, uses an open circuit-eiffel arrangement and contains a $24 \times 24 \times 48$ in. test section. The test section opens up on the bottom and top to allow for the insertion of various test pieces. The tunnel is driven by a $40 \mathrm{hp}$ electric motor that drives a centrifugal fan allowing for wind speeds up to $50 \mathrm{~m} / \mathrm{s}(160 \mathrm{ft} / \mathrm{s})$.
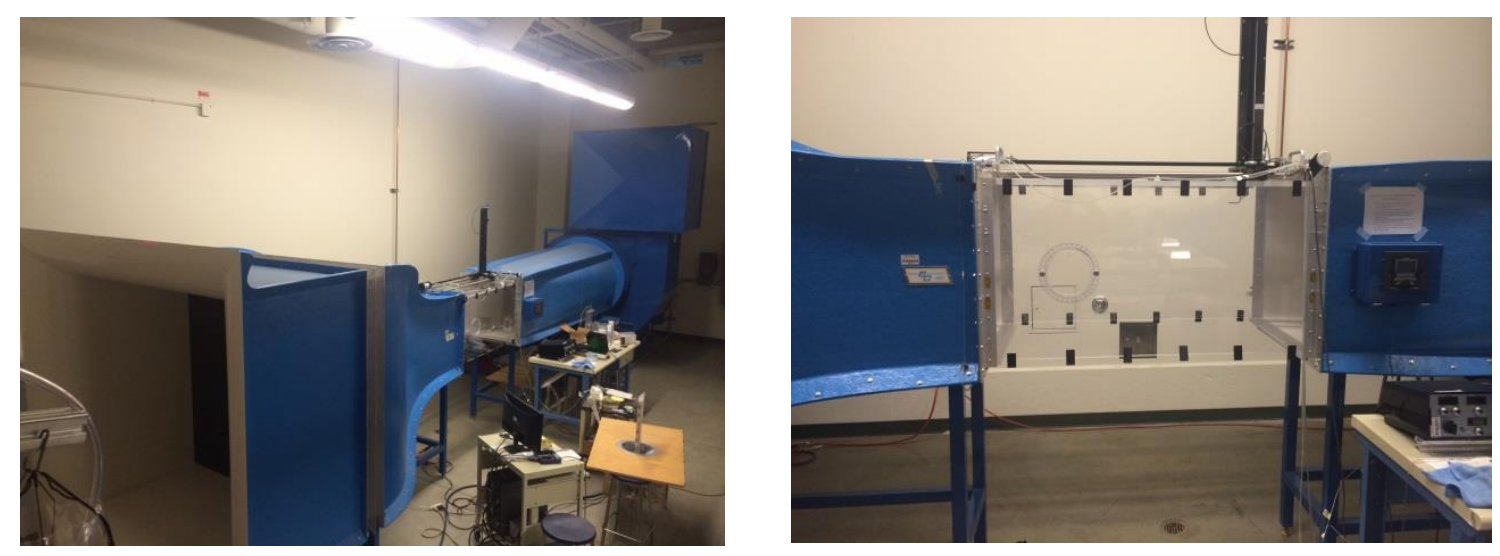

Figure 3.3 Cal Poly $2 \times 2 \mathrm{ft}$ Wind Tunnel 
For this experiment, the bottom of the test section was removed and an insert was designed to hold the test model. This insert is discussed in Section 2.4, which discusses the design and manufacture of the test apparatus support structure.

\subsection{Pressure Measurement System}

It was necessary to take pressure data from the wing's 59 pressure ports. The Scanivalve RAD3200 (Remote A/D) System was used to acquire this data. This system was controlled with LabVIEW (see Section 3.1). The components of this system are outlined in the next few sections

\subsubsection{ZOC33/64Px}

The ZOC33 (zero, operate, and calibrate) contains 64 piezoresistive pressure sensors that are connected to the pressure ports in the model as well as the tunnel static and dynamic pressures. Ports 1-32 used pressure transducers with a range of +- $2.491 \mathrm{KPa}\left(10 \mathrm{inH}_{2} \mathrm{O}\right)$ and ports 33-64 used transducers with a range of +- $249.1 \mathrm{~Pa}(1 \mathrm{psid})$. The ZOC33 contains a resistance thermometer (RTD) to measure the temperature. The analog pressure data and the RTD data are digitized in the RADBASE3200 and sent to the PC where it is converted to temperature corrected engineering units. The ZOC33 is shown in Figure 3.4.

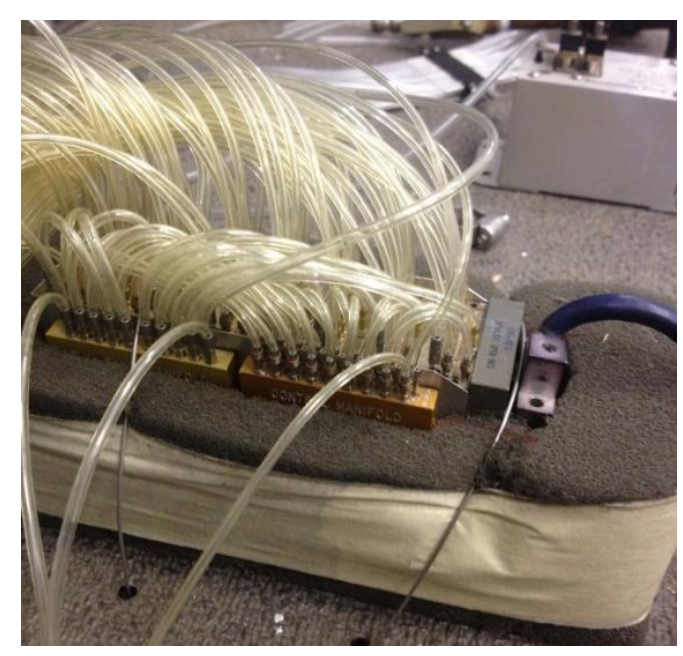

Figure 3.4 ZOC33/64Px 


\subsubsection{RADBASE 3200-EXT}

The RADBASE 3200 is the base unit of the RAD3200 system, which incorporates the USB and power connections, the USB extender, and an on/off switch. It becomes RADBASE 3200EXT when the USB extender is used. RADBASE 3200 contains a 16-bit A/D module, which supports the ZOC33 pressure scanner. The RADBASE 3200 is shown in Figure 3.5.

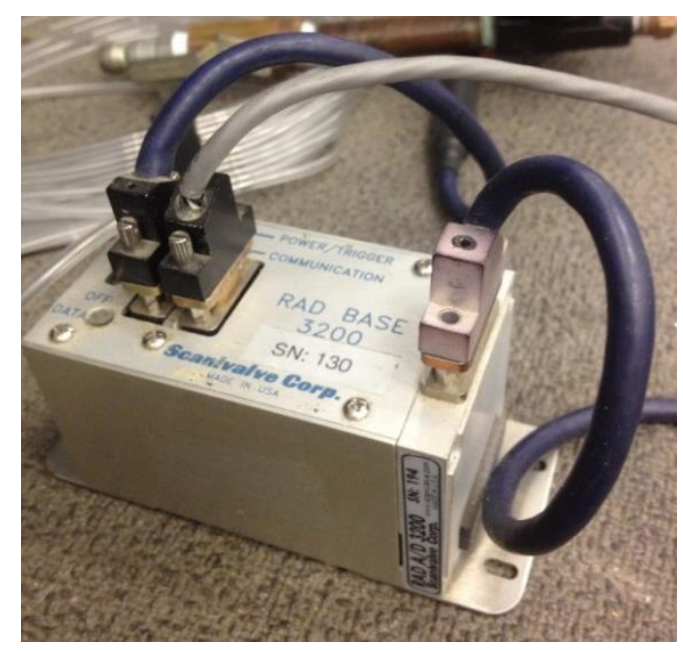

Figure 3.5 RADBASE 3200-EXT

\subsection{Linear Amplifier}

The computer's PCI card could not produce the $180 \mathrm{Vpp}$ signal needed to power the actuators, therefore a 9-volt signal was generated and amplified 20x. The Piezo Systems Inc. EPA-104 Linear Amplifier was used to do this. The amp takes an input up to \pm 10 volt and amplifies it up to $20 x$. This amp has a 40 -watt peak power output, $\pm 200 \mathrm{~mA}$ maximum current, and $\pm 200 \mathrm{Vpp}$ max. The amp is shown in Figure 3.6. 


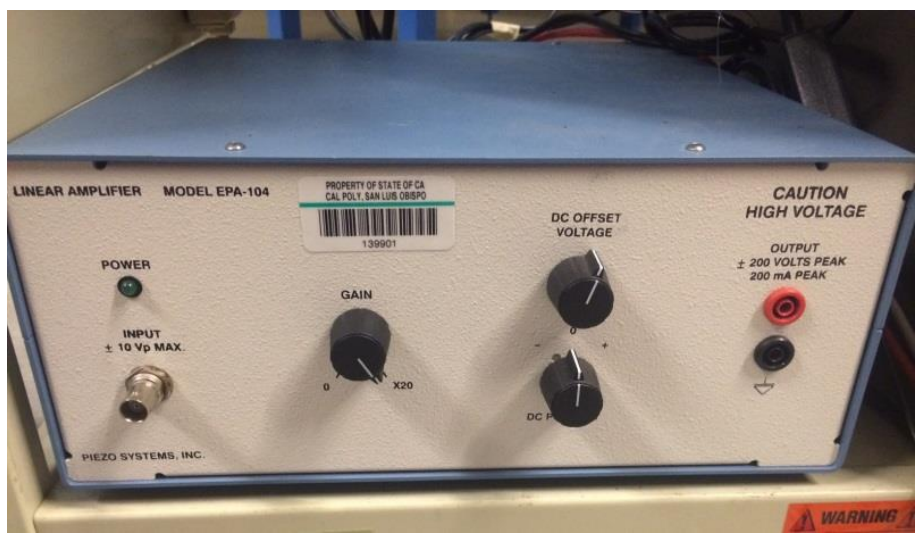

Figure 3.6 EPA-104 Linear Amplifier

\subsection{Bimorph Actuator Disk}

The Bimorph actuator used for the experiment was the Piezo Systems, Inc. T216-A4NO573X. The actuator is made out of two layers of piezoceramic bonded together, which bow in and out like a drumhead. A schematic of the actuator is shown in Figure 3.7.

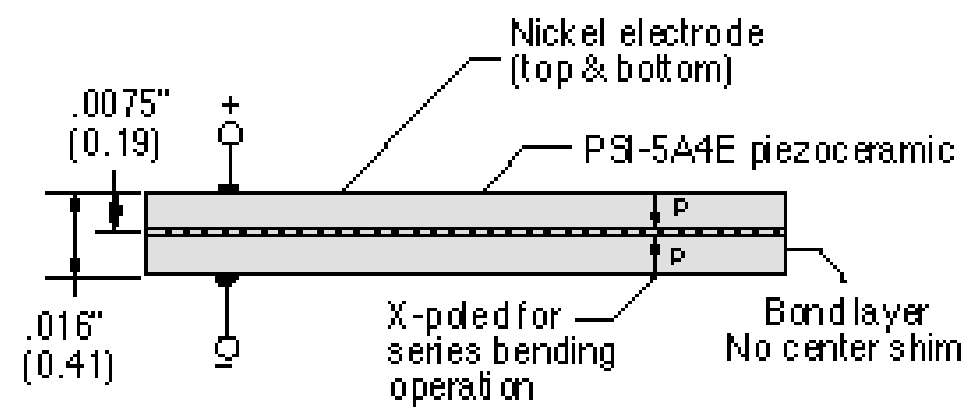

Figure 3.7 Bimorph actuator schematic ${ }^{23}$

The actuator has a 2.5 inch diameter, a weight of 9.8 grams, a $107 \mathrm{nF}$ capacitance, a maximum rated voltage of $\pm 280 \mathrm{Vpp}$, and a resonance frequency of $290 \mathrm{~Hz}$. Because the actuator has no center shim, it is very delicate and careful handling was needed to prevent damage. Several actuators were broken throughout the course of this experiment. One was broken from running it at a resonance frequency, another from a lead wire being tugged too hard, and another due to misalignment of the actuator during clamping. Electric leads were soldered to the actuators using standard flux and solder. 


\subsection{Load Cell}

An Omega LCFD-1KG load cell, shown in Figure 3.8, was used to measure the lift and drag on the wing. This load cell has a $2.2 \mathrm{lb}$. capacity, $5 \mathrm{Vdc}$ excitation, $2 \mathrm{mV} / \mathrm{V}$ nominal output, $0.15 \%$ FSO nominal accuracy, and a $0.15 \%$ FSO nominal repeatability ${ }^{24}$.

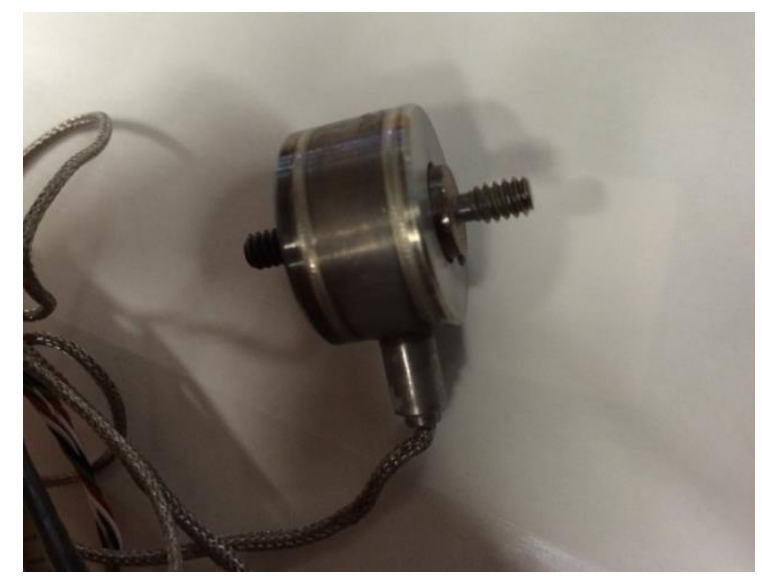

Figure 3.8 Omega LCFD-1KG Load Cell

One end of the load cell was screwed into the support structure and the other end was pressed against the wing support. When the tunnel was turned on the wing support pressed into the load cell where the output voltage was read with LabVIEW.

\subsection{NACA 0015 Semispan Model}

The NACA 0015 Semispan Model was designed and manufactured for this experiment. Specifics about the design and manufacture process can be found in Section 2.3. The model, shown in Figure 3.9, has a semispan of 16.813 inches and a chord of 8 inches. The aspect ratio is 4.20 when based on full span. The model is mounted vertically to the load cell support structure. Details about the load cell support structure can be found in Section 2.4. This support structure was made to measure the lift and drag on the model. 


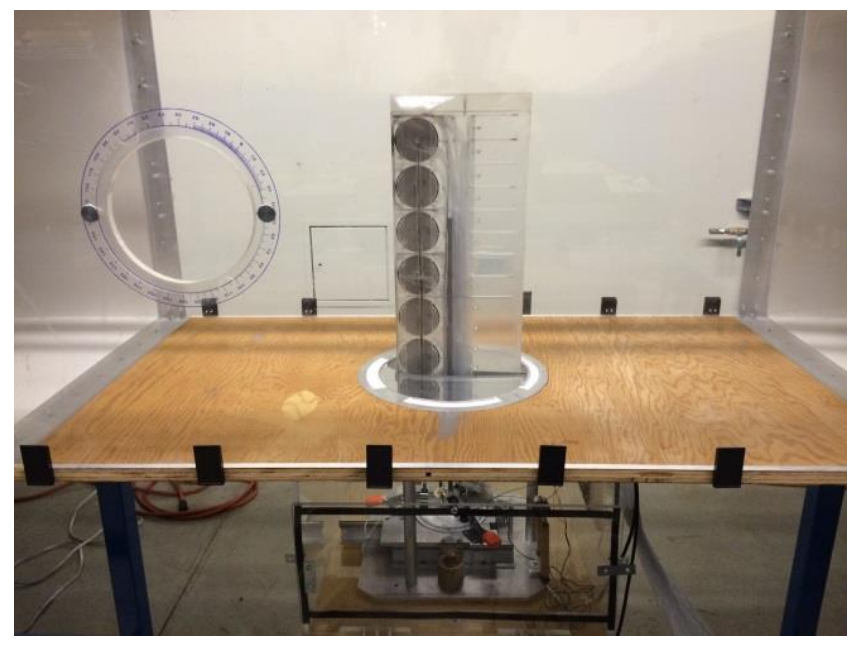

Figure 3.9 NACA 0015 Semispan Model

The model has a 0.03 " wide and a 4.128 " long slit along the top edge of the wingtip. This slit provides synthetic jet actuation in the spanwise (y-axis) direction. The slit is shown in Figure 3.10. Four rows of suction surface pressure ports and two rows of pressure surface pressure ports were added. The pressure port layout is shown in Figure 3.11. The $2 y / b$ locations for the rows A, $\mathrm{C}, \mathrm{D}$, and $\mathrm{F}$ are $37.5 \%, 70.2 \%, 85.1 \%$, and $93.1 \%$ respectively. The $2 \mathrm{y} / \mathrm{b}$ locations for rows $\mathrm{B}$ and $\mathrm{E}$ are $37.5 \%$ and $85.1 \%$ respectively. The pressure port $\mathrm{x} / \mathrm{c}$ locations for rows $\mathrm{A}, \mathrm{C}, \mathrm{D}$, and $\mathrm{F}$ are shown in Table 3.1 and the $\mathrm{x} / \mathrm{c}$ locations for rows $\mathrm{B}$ and $\mathrm{E}$ are shown in Table 3.2.

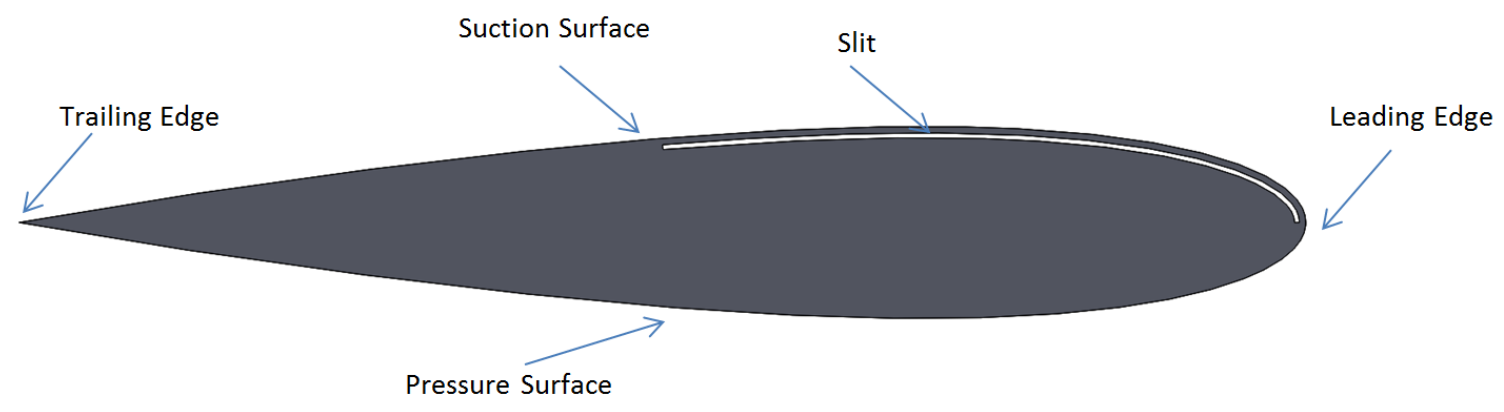

Figure 3.10 Wingtip 

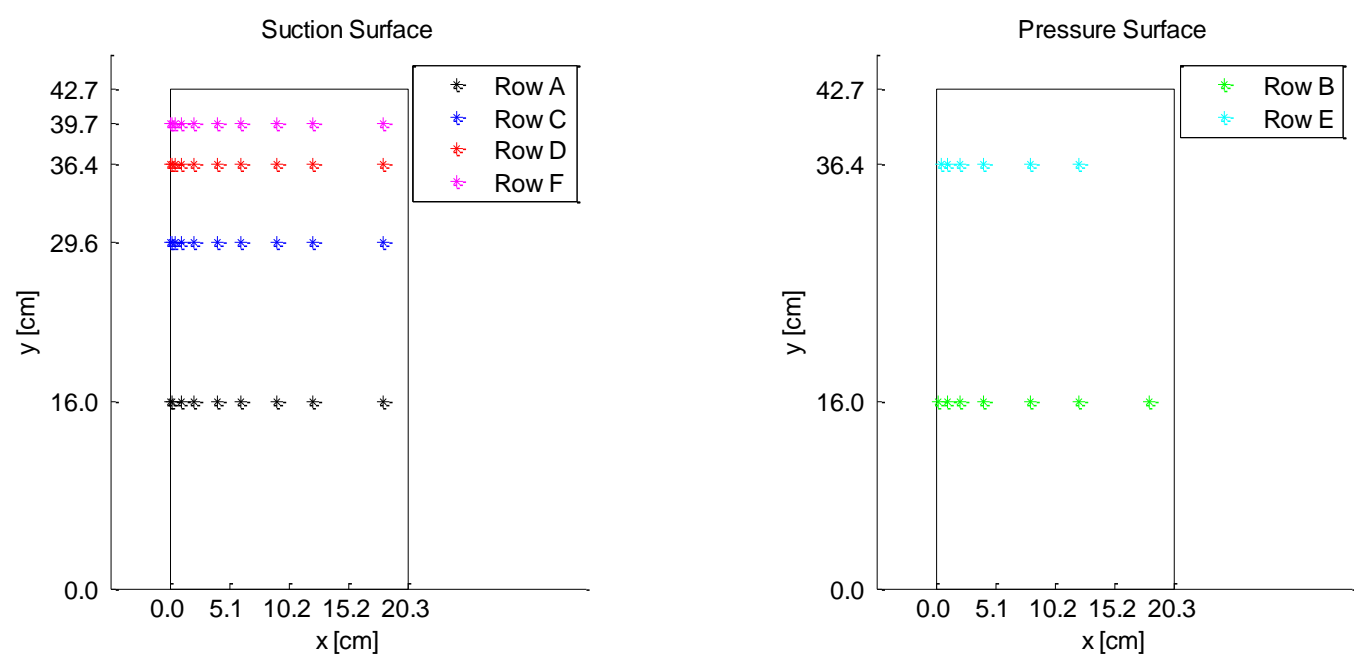

Figure 3.11 Pressure port row locations

Table 3.1 Top pressure port locations

\begin{tabular}{|c|c|}
\hline Port \# & $x / c, \%$ \\
\hline 1 & 0.2 \\
\hline 2 & 0.5 \\
\hline 3 & 1.3 \\
\hline 4 & 2.1 \\
\hline 5 & 4.7 \\
\hline 6 & 10.0 \\
\hline 7 & 20.0 \\
\hline 8 & 30.0 \\
\hline 9 & 45.0 \\
\hline 10 & 60.0 \\
\hline 11 & 90.0 \\
\hline
\end{tabular}

Table 3.2 Bottom pressure port locations

\begin{tabular}{|c|c|}
\hline Port \# & $x / c, \%$ \\
\hline 1 & 1.0 \\
\hline 2 & 2.0 \\
\hline 3 & 5.2 \\
\hline 4 & 10.0 \\
\hline 5 & 20.0 \\
\hline 6 & 40.0 \\
\hline 7 & 60.0 \\
\hline 8 & 90.0 \\
\hline
\end{tabular}

\subsection{Hotwire Anemometer System}

A hot-wire anemometer system was used to measure jet velocities used for the $C_{\mu}$ calculations. The system consists of a TSI Model 1210 hotwire sensor, a Model 1155 Standard Probe Support, an IFA 300 constant temperature anemometer, a DAQ connector, and computer with ThermalPro software. A TSI Model 1127A automatic velocity calibrator was used along with a MKS Baratron Type 220D pressure transducer to do the hotwire calibration. 


\subsubsection{Thermal Anemometer}

An IFA 300 constant temperature anemometer was used in this experiment to measure the velocity profile of the synthetic jet to be used on the momentum coefficient calculation. This anemometer uses a Wheatstone bridge and amplifier circuit that controls a hotwire sensor. Commands are sent to the anemometer using ThermalPro.

\subsubsection{Hotwire Sensor}

A TSI Model 1210-TI.5x hotwire sensor, shown in Figure 3.12, was used for this experiment.

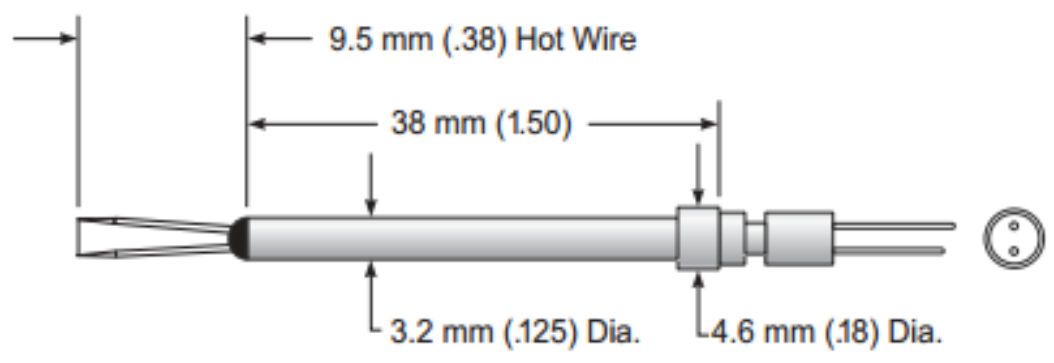

Figure 3.12 Model 1210 General Purpose Probe P $^{25}$

This hotwire is a one-dimensional flow measurement tool that can be used in air up to $150^{\circ} \mathrm{C}$. It has a thin tungsten platinum coated wire with a diameter of $0.00381 \mathrm{~mm}$ and length of $1.27 \mathrm{~mm}$ which changes resistance when exposed to a flow. The specifications are shown in Table 3.3 .

Table 3.3 TSI 1210-TI.5x hotwire sensor specifications

\begin{tabular}{|c|c|c|c|c|c|c|}
\hline $\begin{array}{c}\text { Sensor } \\
\text { No. }\end{array}$ & $\begin{array}{c}\text { Wire Aspect } \\
\text { Ratio }\end{array}$ & $\begin{array}{c}\text { Probe } \\
\text { RES at } \\
0^{\circ} \mathrm{C} \\
R_{0}, \Omega\end{array}$ & $\begin{array}{c}R_{100}-R_{0} \\
\Omega\end{array}$ & $\begin{array}{c}\text { Recommended } \\
\text { Oper. RES } \\
R_{0 p}, \Omega\end{array}$ & $\begin{array}{c}\text { Recommended } \\
\text { Oper. Temp. } \\
T_{0 p},{ }^{\circ} \mathrm{C}\end{array}$ & $\begin{array}{c}\text { Internal } \\
\text { Probe RES } \\
R_{\text {int }}, \Omega\end{array}$ \\
\hline 1 & 333 & 2.88 & 1.03 & 5.46 & 250 & 0.20 \\
\hline
\end{tabular}


The hotwire sensor was attached to a dual sensor TSI Model 1155-6 Standard Probe Support. This support was used for mounting the hotwire during calibration and during fixturing for the $\mathrm{C}_{\mu}$ test.

\subsubsection{Automatic Velocity Calibrator}

A TSI Model 1127A automatic velocity calibrator was used to calibrate the hotwire sensor. A schematic of velocity calibrator system is shown in Figure 3.13. An air supply is connected to a filter/regulator assembly to condition the air entering the calibrator. The pressure is set to 20-30 psi using this assembly. Once the air enters the calibrator, the flow can be adjusted using a fine and coarse knob. A hotwire sensor is mounted vertically on the calibrator in front of a $10 \mathrm{~mm}$ opening out of which the velocity is controlled. A MKS Baratron Type 220D-0100A2B pressure transducer measures the pressure differential of this flow. This differential is sent to the computer and converted to velocity value with ThermalPro. The thermocouple was not used as the temperature of the flow stays nearly constant and a correction was not needed. 


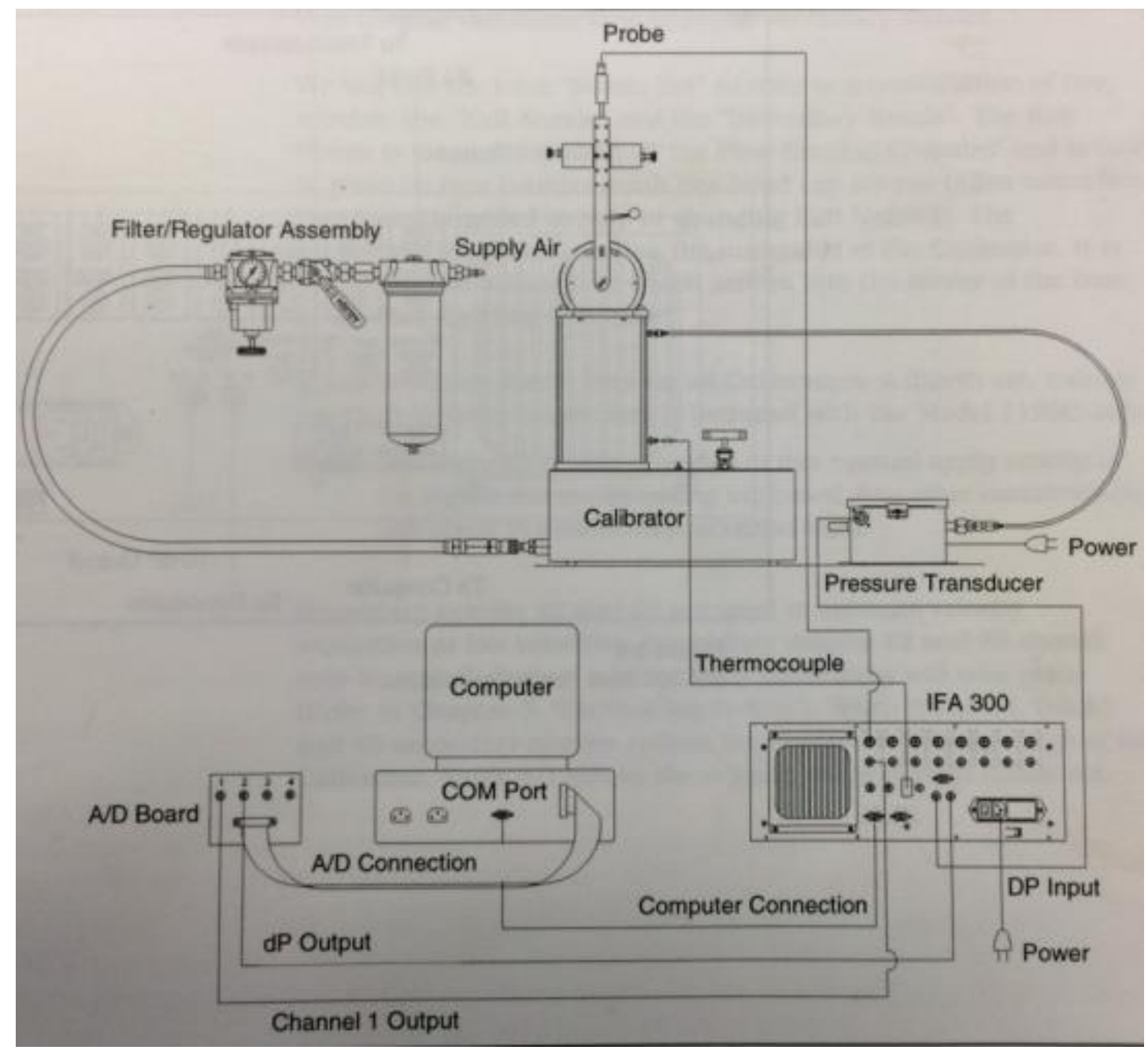

Figure 3.13 Automatic Velocity Calibrator System Schematic

\subsubsection{Hotwire Fixture}

The hotwire was mounted above the wing to measure the synthetic jet velocity profile. Initially velocity measurements were attempted by hand but it was too difficult to get a consistent measurement. A fixture was made, as shown in Figure 3.14, to hold the probe directly above the jet in a fixed location. This fixture consisted of a stand, a traverse, the probe support, and the hotwire sensor. The traverse was mounted to the wooden stand so it was horizontal above the wing. The probe support was placed inside a collet on the traverse. A stopper was placed on the probe support to keep the hotwire sensor about $0.5 \mathrm{~mm}$ above the slit. The traverse was adjusted to move the hotwire to different locations along the width of the slit. To move the hotwire along 
the length of the slit, the stand was moved. A micrometer was used to measure the exact location of the hotwire with respect to the slit.

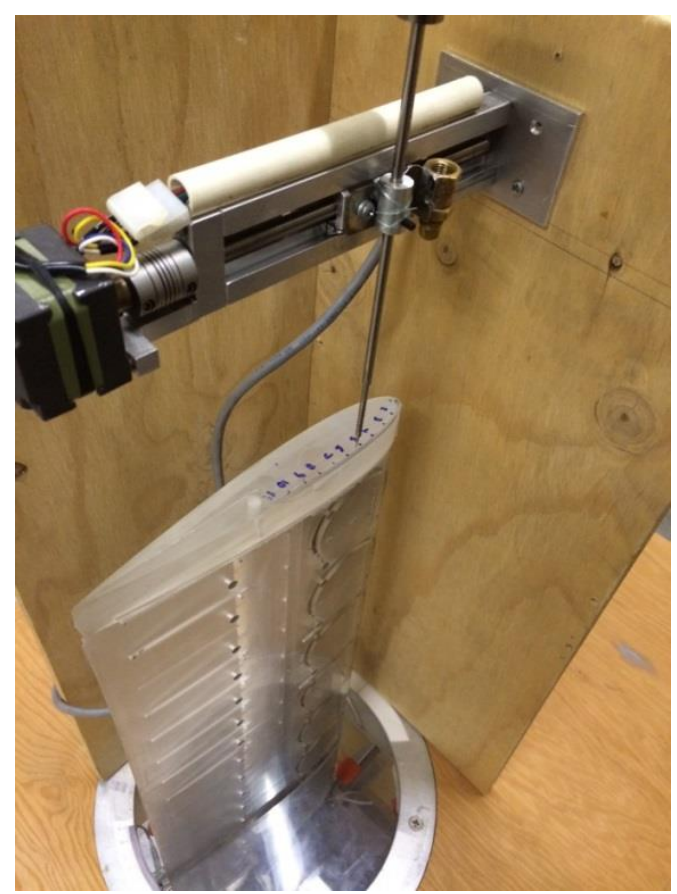

Figure 3.14 Hotwire fixture 


\section{Analysis}

\subsection{Momentum Coefficient Calculation}

The momentum coefficient was calculated to gauge the strength of the synthetic jet. The equation used to calculate the momentum coefficient is,

$$
C_{\mu}=\frac{\rho_{j} A_{s} u_{r m s, j}^{2}}{q_{\infty} S}
$$

where $\rho_{j}$ is the jet density, $A_{s}$ is the slit area, $q_{\infty}$ is the freestream density, and $S$ is the planform area. A hotwire anemometer system was used to measure the synthetic jet velocity profile which was time averaged to get the RMS velocity profile. A detailed explanation of the hotwire test can be found in Section 5.2. The RMS velocity was calculated as,

$$
u_{r m s}=\sqrt{\frac{1}{n}\left(u_{1}^{2}+u_{2}^{2}+\cdots+u_{n}^{2}\right)}
$$

where $\mathrm{u}$ is the instantaneous velocity and $\mathrm{n}$ is the number of samples. The RMS velocity contains the rectified expulsion phases of many jet cycles.

\subsection{Coefficient of Pressure Calculation}

The equation for the pressure coefficient as shown in Equation 4.3 modified to account for tunnel effects as shown in Equation 4.4

$$
\begin{gathered}
C_{p}=\frac{p-p_{\infty}}{q_{\infty}} \\
=\frac{p_{i}-K_{p} p_{\text {tunnel }}}{K_{\varepsilon} K_{q}\left(p_{t, \text { tunnel }}-p_{\text {tunnel }}\right)}
\end{gathered}
$$

Where $p_{i}$ is from the pressure ports, $K_{p}$ is the tunnel calibration correction for the static pressure, $K_{q}$ is the tunnel calibration correction for dynamic pressure, and $K_{\varepsilon}$ is the correction due to wake and solid blockage. These correction factors are obtained in the next few sections. 


\subsubsection{Tunnel Calibration}

The tunnel total pressure is measured just inside the flow straightener. The tunnel static pressure is measured after the exit of the nozzle just before the test section. These pressures are used in the pressure coefficient calculation (dynamic and static pressure is used but total pressure is used to calculate dynamic pressure) although they are slightly different from the static and total pressures in the test section. The total and static pressure in the test section would ideally be used but during the test, the wing model is taking up the place of the test section and a probe cannot be inserted. A calibration was done to correct this problem. The test section pressures were measured using a pitot-static tube and a correction was made using

$$
\begin{gathered}
q_{A}=K_{q} q_{\text {tunnel }} \\
p_{A}=K_{p} p_{\text {tunnel }} \\
p_{t, A}=K_{t} p_{t, \text { tunnel }}
\end{gathered}
$$

Where $\mathrm{K}$ is a correction factor applied to the tunnel pressures and the subscript A denotes the pressures measured by the pitot-static tube. $\mathrm{K}_{\mathrm{q}}, \mathrm{K}_{\mathrm{p}}$, and $\mathrm{K}_{\mathrm{t}}$, were found to be 1.001, 1.012, and 1.025 respectively.

\subsubsection{Solid \& Wake Blockage}

Solid Blockage occurs when walls of the tunnel confine the area that the air stream has to flow around the wing. This essentially pushes the streamlines towards the wing model surface increasing the velocity of the air as it flows around the model. The Solid and Wake Blockages were calculated using the procedures given in Barlow ${ }^{26}$. The equation for the solid blockage is given as

$$
\varepsilon_{s b}=\frac{K \tau v}{C^{3 / 2}}
$$


Where $\mathrm{K}$ is the body shape factor, $\tau$ is a parameter based on figure 10.3 in $\mathrm{Barlow}^{26}, \mathrm{~V}$ is the volume of the model, and $\mathrm{C}$ is the test section area. The solid blockage in this experiment was found to be 0.0073 therefore there is only a $0.73 \%$ increase in velocity due to the presence of the wing.

The wake blockage is similar to the solid blockage except it is due to the wake of the model. In free air conditions, the flow would be able to expand around the wake. Because there are tunnel walls, the flow cannot expand and must speed up to get past the wake. The correction for this is

$$
\varepsilon_{w b}=\frac{S}{4 C} C_{D 0}+\frac{5 S}{2 C}\left(C_{D u}-C_{D i}-C_{D 0}\right)
$$

Where $C_{D u}$ is the uncorrected drag coefficient, $C_{D i}$ is the induced drag coefficient, and $C_{D 0}$ is the zero angle of attack drag coefficient. If $\left(C_{D u}-C_{D i}-C_{D 0}\right)$ is less than zero the whole term is set to zero. This term is used to account for separated flow and a negative value would mean that the flow is not separated. The wake blockage is different for each angle of attack. For $\alpha=10$ degrees at $\operatorname{Re}=100,000$, the wake blockage is 0.0047 .

The solid and wake blockage are used to correct the freestream dynamic pressure by using

$$
\begin{gathered}
q_{c}=q_{A} K_{\varepsilon} \\
K_{\varepsilon}=\left(1+\left(\varepsilon_{s b}+\varepsilon_{w b}\right)\right)^{2}
\end{gathered}
$$

Where $K_{\varepsilon}$ is the correction factor applied to the actual freestream dynamic pressure.

\subsubsection{Buoyancy Correction}

The tunnel static and dynamic pressure varies across the length of tunnel. This is because the boundary layer grows in the tunnel walls as the air flows through the tunnel. This thickening

of the boundary layer decreases the effective wind tunnel cross section increasing its speed if the actual cross section remains fixed. A well designed tunnel will have a minimal increase in speed 
along its length. This increase in speed increases the drag on the model, hence the need for a buoyancy correction. The buoyancy corrections were calculated using the procedures given in Barlow $^{26}$. The equation for this correction is,

$$
\Delta D_{B}=-\frac{\pi}{4} \lambda_{3} t^{3} \frac{d p}{d l}
$$

Where $\lambda$ the body shape factor and $\mathrm{t}$ is the wing thickness. Dp/dl was calculated by running a pitot-static probe along the length of the test section and seeing how the pressure varied. A plot of this variation is shown in Figure 4.1
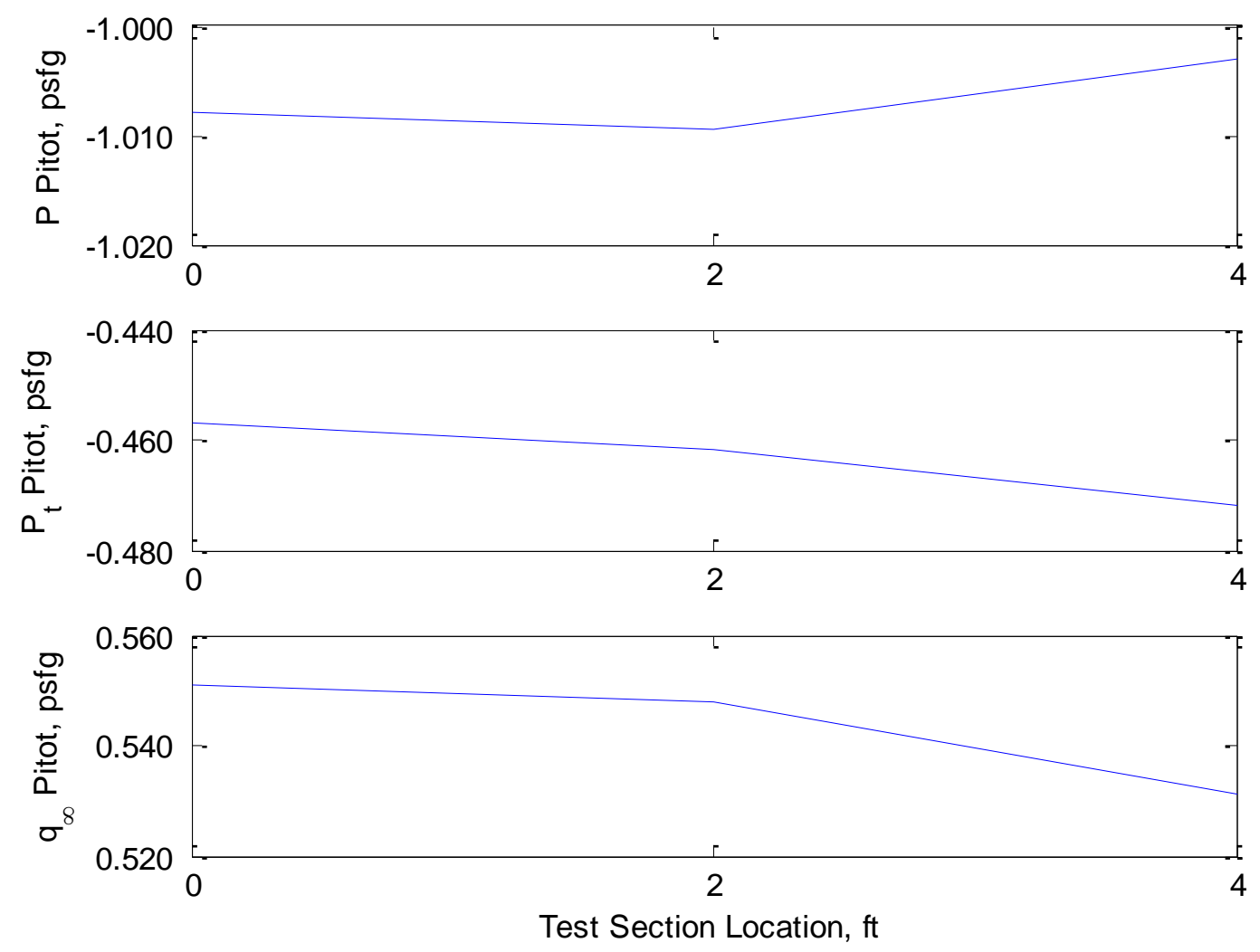

Figure 4.1 Wind tunnel lengthwise variation in static pressure, total pressure, and dynamic pressure

$\mathrm{Dp} / \mathrm{dl}$ was calculated to be $8.6 \mathrm{e}-6 \mathrm{psi} / \mathrm{ft}$, which is negligible. The using this value, the buoyancy corrections comes out to $2.34 \mathrm{e}-08$. This value can be neglected because is a few orders of magnitude smaller than the error in the drag. 


\subsection{Sectional Force Coefficients}

The model contains two complete rows of pressure ports at $2 \mathrm{y} / \mathrm{b}=37.5 \%$ and $85.1 \%$. These are called "Rows A \& B" and "Rows D \& E" respectively. A pressure integration was done to obtain the section lift coefficient at each of these rows. The normal and axial coefficient forces can be found using a simple panel method. The panels are set up such that each pressure port on the airfoil is either the start or end of the panel as shown in Figure 4.2 where the points depict the locations of the pressure ports.

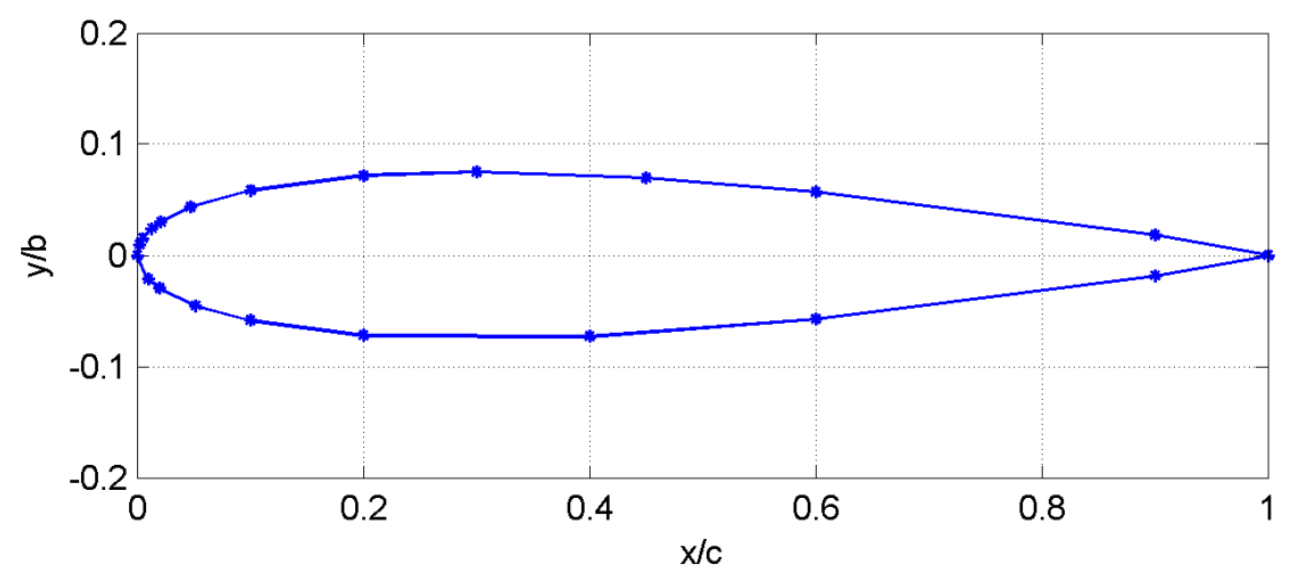

Figure 4.2 Panels used in pressure integrations

Knowing the pressure coefficient, $\mathrm{x}$ and $\mathrm{y}$ locations of the pressure ports on the airfoil, the normal and axial forces and be represented as a sum of these pressures along the entire airfoil. The normal and axial force coefficients are,

$$
\begin{gathered}
c_{n}=-\sum_{\text {panels }}\left(\frac{C_{p i}+C_{p i+1}}{2}\right)\left(\frac{x_{i+1}}{c}-\frac{x_{i}}{c}\right) \\
c_{a}=\sum_{\text {panels }}\left(\frac{C_{p i}+C_{p i+1}}{2}\right)\left(\frac{y_{i+1}}{c}-\frac{y_{i}}{c}\right)
\end{gathered}
$$

where $\mathrm{i}$ is the port index, $\mathrm{x}$ is the $\mathrm{x}$-location of the pressure port, $\mathrm{y}$ is the $\mathrm{y}$-location of the pressure port, and the pressure coefficients are averaged along the panel. The lift and drag can be found using the normal and axial forces on the airfoil. The lift and drag coefficient can be written 
in terms of the normal and axial coefficients and angle of attack. The lift and drag coefficients are,

$$
\begin{aligned}
& c_{l}=c_{n} \cos \alpha-c_{a} \sin \alpha \\
& c_{d}=c_{n} \sin \alpha+c_{a} \cos \alpha
\end{aligned}
$$

where $\mathrm{c}_{\mathrm{n}}, \mathrm{c}_{\mathrm{a}}$, and $\alpha$ are normal and axial forces and angle of attack respectively.

\subsection{Direct Load Measurement}

An Omega LCFD-1KG load cell was used to measure the aerodynamic loads on the wing. Before this could be done, the load cell had to be calibrated. The calibration was done by hanging a series of weights from the wing and measuring the output. LabVIEW was used to measure the output. Each reading consisted of 30,000 samples at a $10,000 \mathrm{~Hz}$ sample rate. A plot of the calibration curve is shown in Figure 4.3.

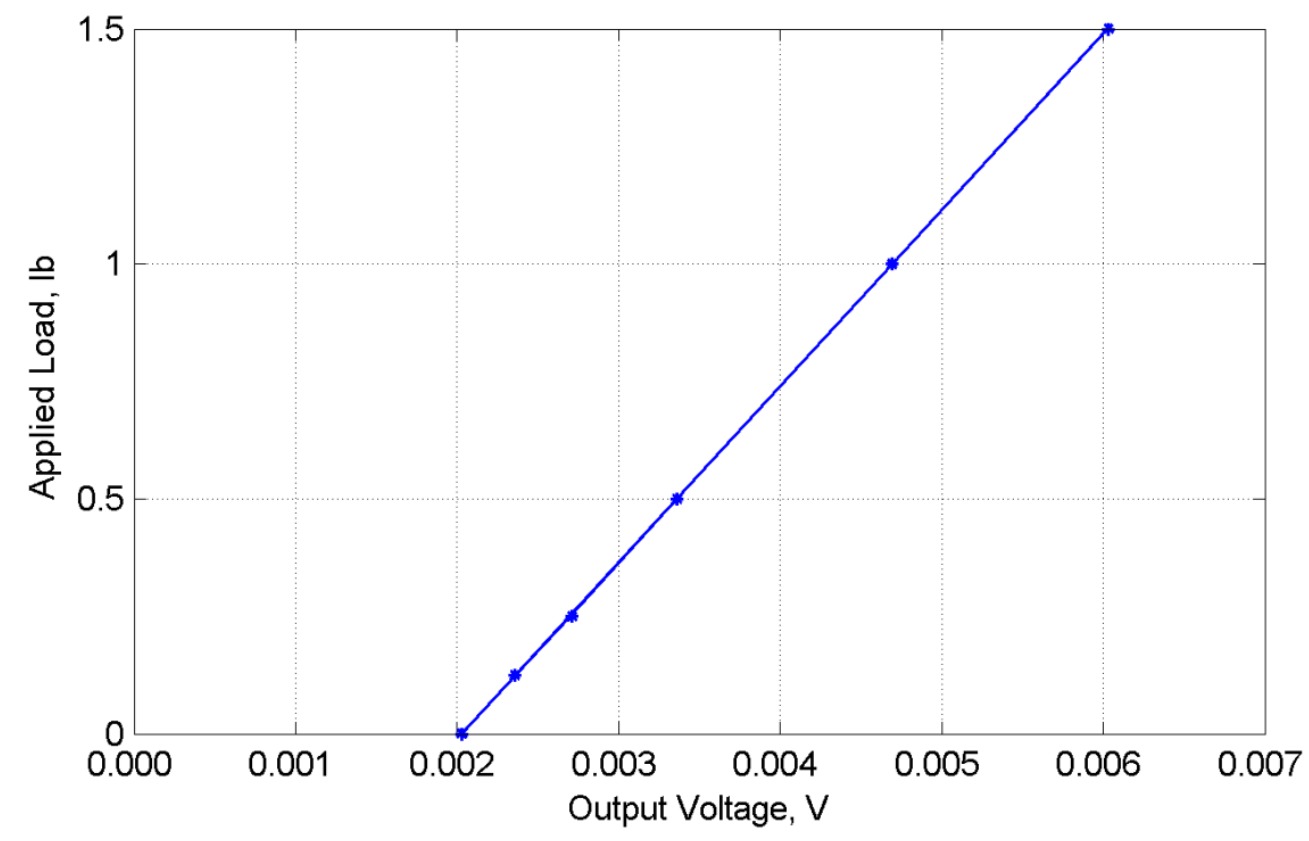

Figure 4.3 Load cell calibration curve

The weights used were $0,1 / 8,1 / 4,1 / 2,1,1.5 \mathrm{lb}$. The plot is linear with an R-squared value of 1 . The equation of the calibration curve is 


$$
F=375.32 V+0.7267
$$

Where $\mathrm{F}$ is the applied load and $\mathrm{V}$ is the voltage. The lift and drag on the wing will be calculated using this equation. A $1 \mathrm{lb}$. weight was hung from the bearing plate to pull the plate into the load cell. This preload was meant to protect the load cell by preventing any small vibrations from slamming the plate into the load cell. The y-intercept does not read 1lb because the weight was hung at an angle. The weight was hung this way due to space restrictions inside the airtight case. The weight was hung exactly the same orientation for each test. To change from measuring lift to measuring drag, the load cell support structure is rotated 90 degrees. This calibration was done with the load cell in the lift position. A calibration was also done with the load cell in the drag position and the results were identical, therefore, this curve could be used for both load cell orientations.

The lift coefficient and drag coefficients were calculated using

$$
\begin{gathered}
C_{L}=\frac{L}{q_{\infty} S} \\
C_{D}=\frac{D}{q_{\infty} S}
\end{gathered}
$$

Where $\mathrm{L}$ is the lift, $\mathrm{D}$ is the drag, $q_{\infty}$ is the corrected freestream dynamic pressure and $\mathrm{S}$ is the planform area. Note that the planform area is in this equation is based on the half wing

\subsection{Uncertainty Analysis}

Three major groups of data were taken for this experiment: Hotwire data to calculate the momentum coefficient, pressure data to view the pressure distribution and the sectional lift coefficients, and load cell data to measure the lift and drag on the wing directly. Uncertainty analysis was necessary to ensure the reliability of the data used in this experiment. The uncertainty can be expressed as 


$$
\delta q=\sqrt{\left(\frac{\partial q}{\partial x_{1}} \delta x_{1}\right)^{2}+\left(\frac{\partial q}{\partial x_{2}} \delta x_{2}\right)^{2}+\cdots+\left(\frac{\partial q}{\partial x_{n}} \delta x_{n}\right)^{2}}
$$

Where the $\delta q$ is the uncertainty or error in the measurement. Detailed derivations, calculations, and explanations of how the error for each group of data wwas obtained are shown in Appendix 7B. 


\section{Experimental Procedure}

\subsection{Hotwire Calibration}

In order to get accurate velocity readings from the hotwire, it needed to be calibrated. This was done using the automatic velocity calibrator. To begin the calibration, the probe was mounted to the calibrator. The probe can be mounted to the calibrator in several orientations. For this experiment, it was mounted in the vertical position since that is how it will be used for the momentum coefficient calculation. Next, the shop air supply was connected and ThermalPro was started. In ThermalPro, the maximum and minimum velocities expected in the experiment were defined as $50 \mathrm{~m} / \mathrm{s}$ and $0 \mathrm{~m} / \mathrm{s}$ respectively. After this, the cable resistance and probe tare resistance were measured. ThermalPro was then used to develop a table with velocity points for calibration, which were clustered near high gradient areas in the calibration curve. The hotwire resistance would then need to be measured at each of these velocity points. This was done by adjusting the fine and coarse knobs on the calibrator until the velocity of the calibrator matched the velocity in the table for each point. The MKS Baratron Type 220D-0100A2B pressure transducer was used to measure upstream pressure used to back out the calibrator velocity. Figure 5.1 shows the calibration curve that was obtained though the hotwire calibration. The values in the top right of the calibration curve show the constants for the $4^{\text {th }}$ order polynomial calibration curve. 


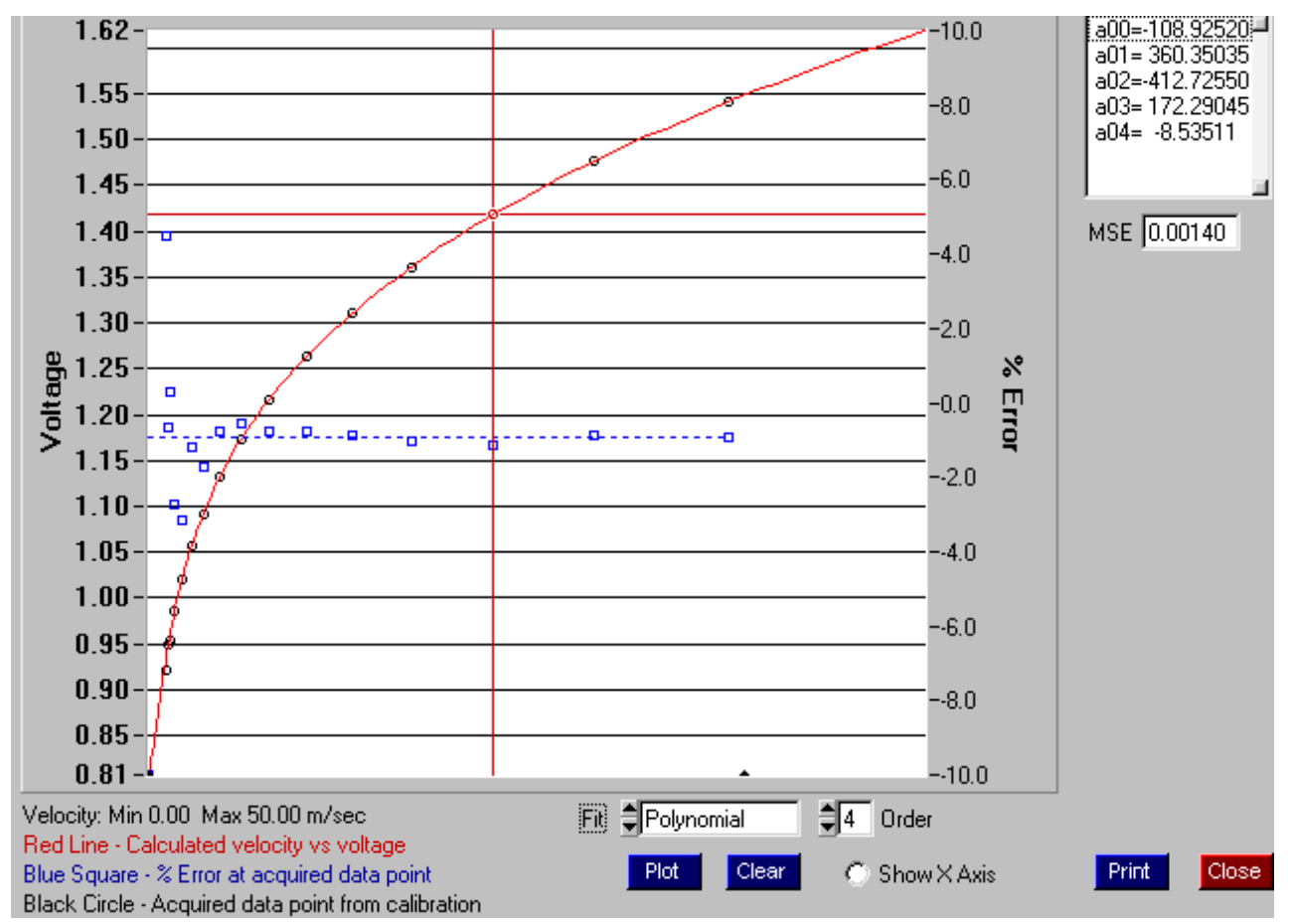

Figure 5.1 Hotwire calibration curve

\subsection{Hotwire Test Matrix}

It was necessary to know how the synthetic jet RMS velocity scales with voltage at each frequency. If this was known, the signal amplitude could be tuned to adjust $\mathrm{C}_{\mu}$ to the needed value given Reynolds number and $\mathrm{F}^{+}$. The hotwire anemometer system was used to measure the synthetic jet velocity profile to make this possible. The actuators were driven by a $0-180 \mathrm{Vpp}$ square wave. The signal was generated with LabVIEW at 0-9 Vpp then amplified 20x using a linear amplifier. A square wave was used because it produces the highest peak velocity compared to the sine and saw tooth wave $\mathrm{e}^{21}$ and the maximum voltage was chosen because this is the actuator maximum rated voltage. The frequency of the square wave was limited to $180 \mathrm{~Hz}$ to avoid actuating near the $290 \mathrm{~Hz}$ actuator resonance frequency, as this would damage the devices. The hotwire probe support was mounted to a traversing system. This was used to hold the hotwire $0.5 \mathrm{~mm}$ above the slit and to test different locations along the slit. The test matrix is shown in Figure 5.2 


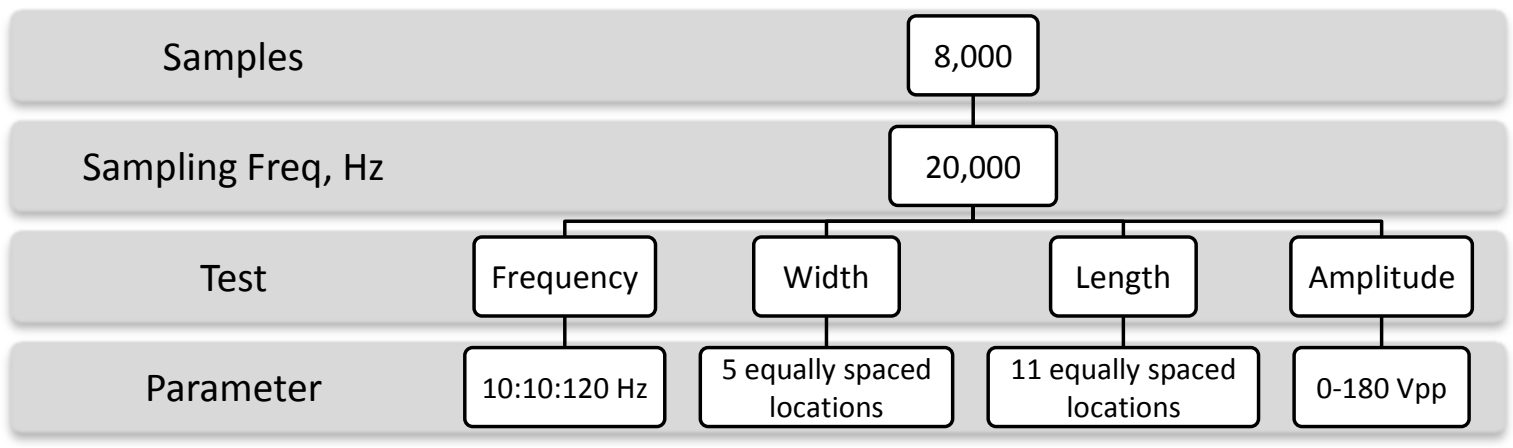

Figure 5.2 Hotwire test matrix

Each acquisition consisted of 8,000 samples at a $20,000 \mathrm{~Hz}$ sampling frequency. Four different tests were completed. For the frequency test, the hotwire was placed near the center of the slit and acquisitions were taken at actuator frequencies every $10 \mathrm{~Hz}$ from 10 to $120 \mathrm{~Hz}$ at 180 Vpp. For the width test, the hotwire was placed just inside the slit and moved across the 0.04" width in 5 equally spaced increments taking data at each location. For the length test, data was acquired in eleven equally-spaced locations along the length of the slit starting at the side nearest to the leading edge and ending on the side closest to the trailing edge. For this test, the hotwire was placed at half-width at each location. For the amplitude test, the actuators were excited at 100 $\mathrm{Hz}$ and the hotwire was placed at the center of the slit. The voltages tested were 2, 3, 4, 10, 20, $40,60,80,100,120,140,160$, and $180 \mathrm{Vpp}$ to see how the RMS velocity profile scales with amplitude. Using this data, an interpolation could be performed to find $\mathrm{C}_{\mu}$ at a given $\mathrm{F}^{+}$and Reynolds number.

\subsection{Wing and Load Cell Apparatus Preparation}

Before the wind tunnel test could begin, the wing model and the load cell apparatus needed to be prepared. First, the suction surface slits used for separation control were covered with scotch tape. A smooth layer of wax was applied in to any rough areas on the model covering features such as screw holes, leading and trailing edges, and the junction between the wingtip and the wing. The rails were then lubricated with LPS to minimize static friction in the bearings. A 
weight was hung off a pulley inside the airtight case to pull the wing into the load cell. This was done to prevent the wing from slamming into the load cell and damaging it as soon as the tunnel is turned on. After this, the pressure ports were all checked for leaks by covering the port and creating a vacuum with a vacuum pump. There was no leak if the vacuum held. After this, the wind tunnel insert containing the wing was put into the tunnel and any gaps where air could leak into the tunnel were sealed with scotch tape.

\subsection{Calibrating Lift and Drag Directions}

It was necessary to align the rails perpendicular to the freestream when measuring lift and parallel to the freestream when measuring drag. Geometric relations were used between the tunnel walls, the rotating support, and the wooden insert to make markings that would align the rails. These tick marks were placed on the rotating support and the wooden insert. When the tick marks were aligned, the rails were properly oriented. Before each test, the apparatus was checked to make sure the tick marks were aligned.

\subsection{Angle of Attack Calibration}

Before the start of the test, the zero lift angle of attack was found. Angle of attack tick marks were printed on a piece of paper and glued to the rotating support. Because the wing is symmetric, the zero lift angle of attack is zero. This angle was found by measuring the lift on the wing using the load cell. The angle of attack was adjusted until one was found that did not result in any lift. Once this angle was found, a razor blade was inserted between both halves of the wing. The wing was designed so that the junction of the two halves forms the chord line. The edge of the razor was used to mark the zero. All following angles used in the test were referenced from this mark. 


\subsection{Wind Tunnel Test Matrix}

The goal of the first test was to get the sectional lift coefficient in two locations along the span to see how the lift and drag change due to the synthetic jet. The primary independent test variables were Reynolds number, angle of attack, and excitation amplitude. The dependent variables of interest were the lift, drag, and the sectional lift coefficients. Each load cell acquisition consisted of 30,000 samples a sample rate of 10,000 Hz. Each pressure scan consisted of 400 frames with each frame consisting of 5 averaged data points at a period of 500 . The test matrix is shown in Figure 5.3

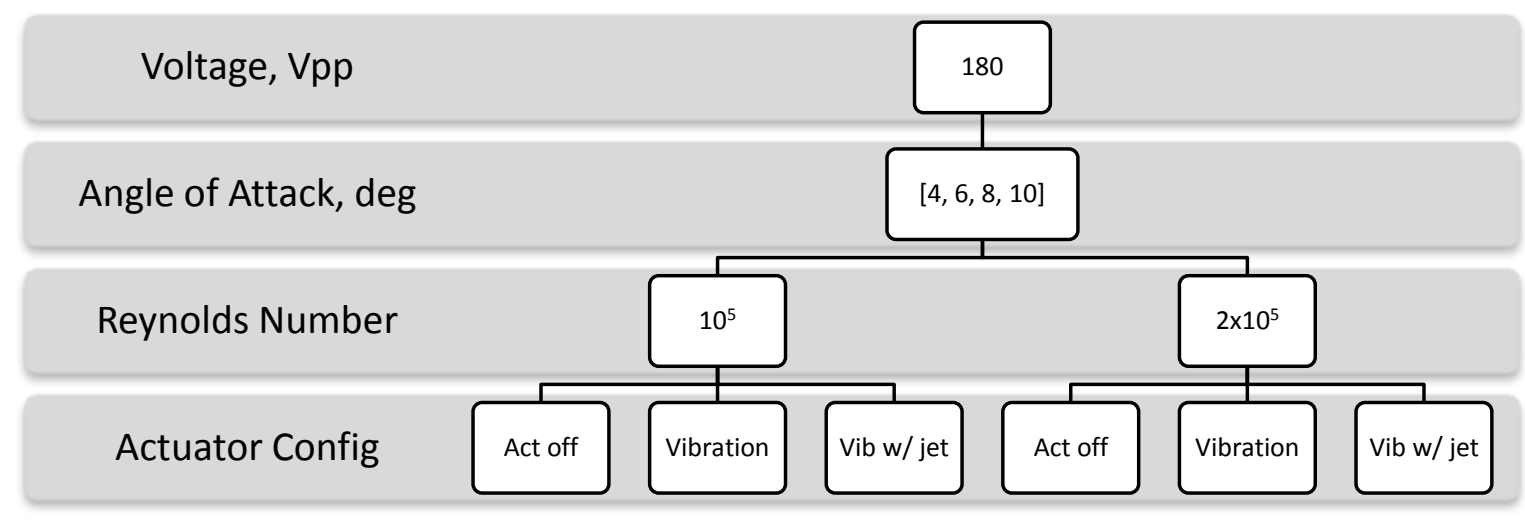

Figure 5.3 Wind tunnel test matrix \#1

The actuators were run at their maximum rated voltage, which is $180 \mathrm{Vpp}$. The 4 angles of attack were chosen to obtain a linear spread for the lift curve. Two Reynolds numbers were chosen. $10^{5}$ was chosen because this is what a previous study used. $2 \times 10^{5}$ was chosen to decrease the error bars in the load cell and pressure data although $\mathrm{C}_{\mu}$ was also lowered which meant less effect on the flow. The idea was to see if increasing Reynolds number could provide better data. The actuator configuration was either "act off", "vibration", or "vib w/ jet". "Act off" means that the actuators are not being powered and no actuation exists. "Vibration" means the actuators are on although scotch tape is being used to cover the slit. When the slit is covered, a relief valve is opened to relief the cavity pressure. "Vib w/ jet" means that the slit is untaped, the relief valve is closed, and that a synthetic jet is emitted from the wingtip. The repeatability was checked by 
repeating all of the $\alpha=4$ degree $\operatorname{Re}=10^{5}$ points and all of the $\alpha=8$ degree $\operatorname{Re}=2 \times 10^{5}$ points. $\mathrm{F}^{+}$ was not kept constant with Reynolds number variation. It was found that $\mathrm{F}^{+}$did not affect the flow field around the wing and that $\mathrm{C}_{\mu}$ was a much more effective parameter ${ }^{12}$. Not holding $\mathrm{F}^{+}$ constant proved benefitial for this test because larger $\mathrm{C}_{\mu}$ values could be obtained since the actuators performed best at $100 \mathrm{~Hz}$ and any deviation from $100 \mathrm{~Hz}$ (necessary to keep $\mathrm{F}^{+}$ constant) would decrease the $\mathrm{C}_{\mu}$. The $\mathrm{F}^{+}$calculation is shown in Appendix A

The next test matrix served to assess the effect of momentum coefficient on the flow. Only pressure data was acquired for this test. The RAD3200 settings were not changed from the previous test. The test matrix is shown in Figure 5.4

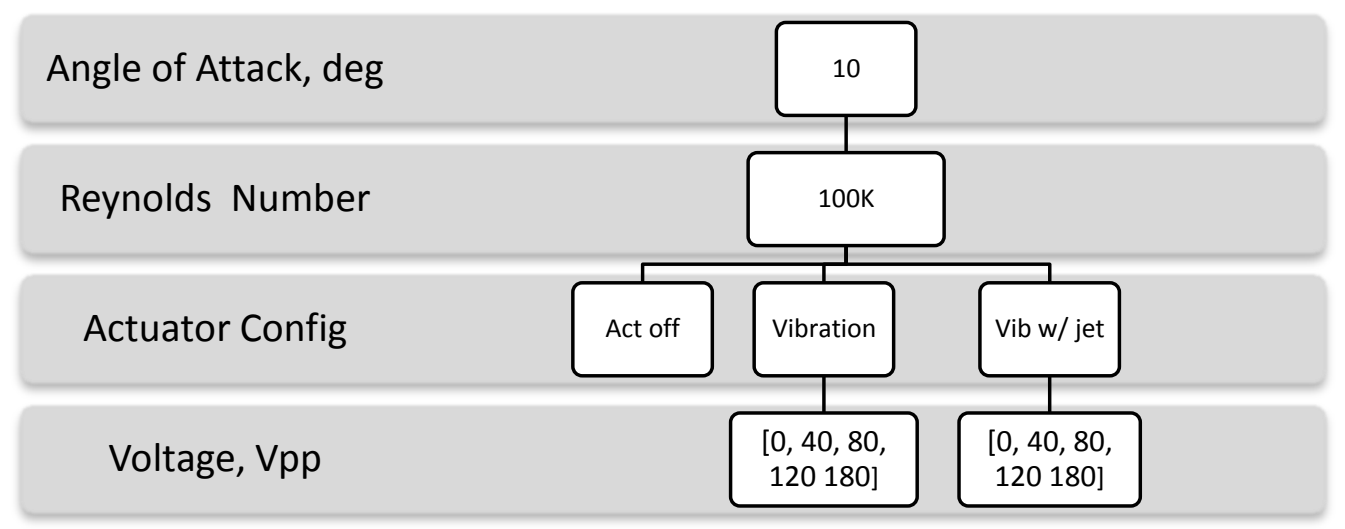

Figure 5.4 Wind tunnel test matrix \#2

The angle of attack of 10 degrees and Reynolds number of $10^{5}$ were chosen because this was where actuation was believed to have the greatest effect. The "Act off", "Vibration" and "Vib w/ jet" cases at $180 \mathrm{Vpp}$ were used as repeats for the previous test. The rest of the cases were used to see how the effect of actuation scales with $\mathrm{C}_{\mu}$.

\subsubsection{Additional Wind Tunnel Test Considerations}

Several aspects of this test could lead to erroneous data if not properly addressed. Many of these were found through trial and error. These aspects are discussed in this section. The model protruded from the cover plate into the tunnel. An 1/8 inch thick gap exists between the cover 
plate and the wing so that the wing does not touch the cover plate and distort the load cell data. When the angle of attack is changed, the cover plate needs to be rotated as well so that it does not touch the model and distort data. Also, at Re>200,000 the model starts to deflect slightly. At these Reynolds numbers, one must look under the tunnel to see if the model is touching the cover plate when the tunnel is on. The case and cover plate were made clear for these reasons. The next aspect of this test that could lead to erroneous data is the airtight case. Care must be taken so that no air leaks into the case. At Re>200,000 one can hear the air entering the case through the gaps if the case in not sealed properly. The air flows in the tunnel through the $1 / 8$ inch gap between the cover plate and the model and can significantly affect data.

\subsection{Flow Visualization}

Oil droplet flow visualization was performed to see if the effect of SJA on the tip shear stress distribution. Mylar tape was placed over the wingtip on the suction surface, pressure surface, and tip surface. Droplets of Corning oil with nominal viscosity of $50 \mathrm{cSt}$ were placed on the tape before the tunnel was turned on as shown in Figure 5.5.

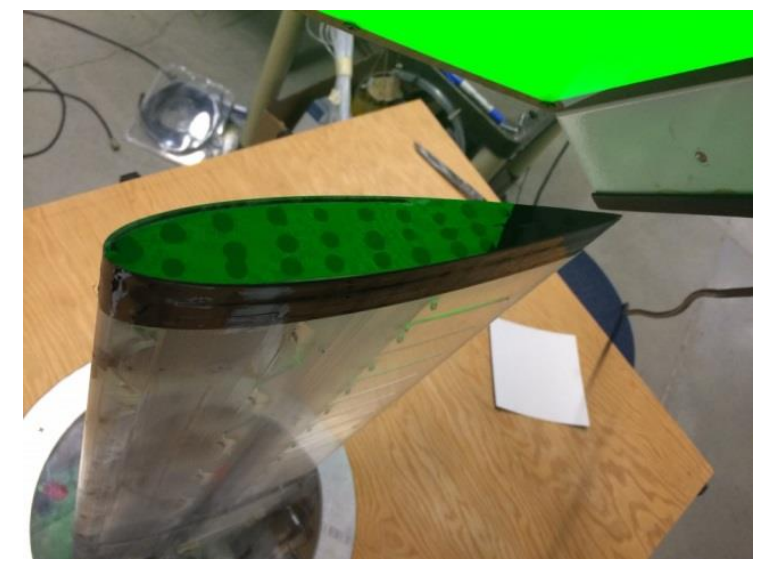

Figure 5.5 Oil droplet distribution used for flow visualization

A green monochromatic light was used to see the droplets more clearly. Due to gravity, the droplets on the pressure and suction surfaces turned into streaks. This turned out to be fine for the 
experiment. Once the tunnel is turned on the oil droplets become thinned out showing the footprint of wing tip vortex on the wing surface as well as the flow direction.

\subsection{Stethoscope Boundary Layer Investigation}

A stethoscope can be used to evaluate the state of the boundary layer to see if it is turbulent or laminar. The turbulent boundary layer was distinguished from the laminar boundary layer by listening to the flow. When the flow was turbulent, pressure fluctuations due to turbulence could be heard in the boundary layer. This test was done to see if vibration was tripping the flow and to ensure boundary layer transition was not occurring in different locations for the "act off", "vibration" and "vib w/ jet" test conditions. The test was done at $\operatorname{Re}=100,000$ and $\operatorname{Re}=200,000$ at $\alpha=10^{\circ}$. Figure 5.6 shows an image taken during the test

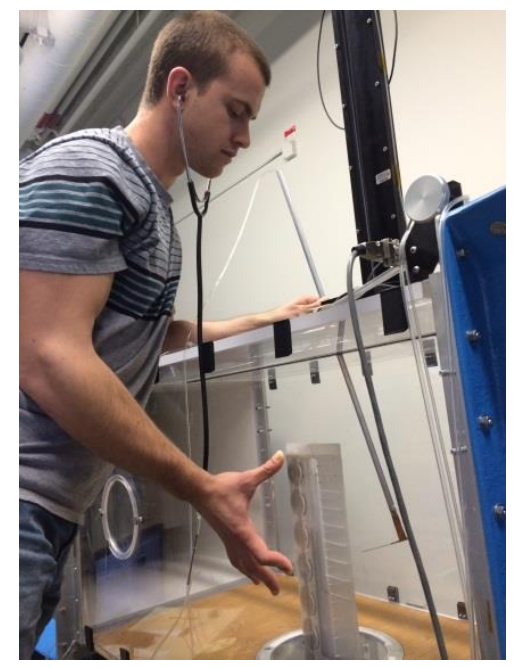

Figure 5.6 Stethoscope boundary layer investigation 


\section{$6 \quad$ Results and Discussion}

First, the momentum coefficient results are shown where a hotwire was used to measure the jet velocities to observe how the synthetic jet behaves and to calculate the momentum coefficient. After this, the baseline data for the wing is compared to existing data for similar wings. The quality of the data is also discussed in this section. Next, the results obtained by analyzing the pressure data are discussed. These results show both the synthetic jet and vibration affect the pressure distribution on the wing. The sectional lift coefficients were also obtained at two $2 \mathrm{y} / \mathrm{b}$ locations. The effect of vibration and the jet on these coefficients are discussed. The momentum coefficient is also varied and the effect on the coefficients can be seen with varying $\mathrm{C}_{\mu}$. Finally, the load cell results are shown which demonstrate the effect of vibration and synthetic jet on the lift and drag of the wing.

Error bars were not included in the plots to avoid clutter. Appendix B shows sample plots with error bars included.

\subsection{Baseline Data Validation}

Validation of the data obtained in this study was a difficult task because of the limited available data. Pressure, lift, and drag data was necessary although most studies focus on much higher Reynolds numbers and use 2-D wings. A few studies that were found used wings that are very similar although not exactly the same as the one used in this study. Because exact data could not be obtained for our specific model, most of this validation serves to show that the data obtained in this Thesis falls into a range that would be expected for a wing of this type. Figure 6.1 shows the lift curve for a NACA 0015 2D wing compared against 3D data from this experiment. 


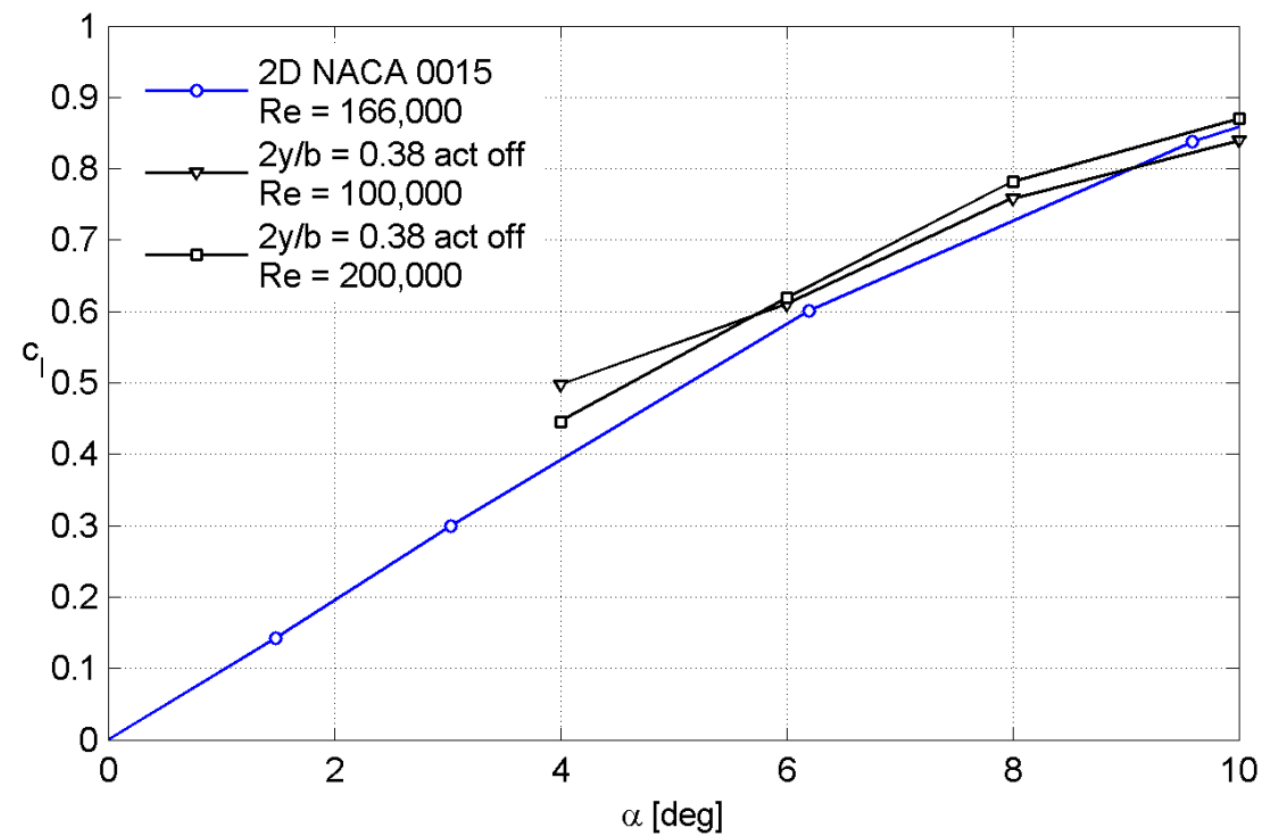

Figure 6.1 Rows A \& B baseline sectional lift compared to 2D NACA 0015 airfoil data $^{27}$

Figure 6.1 shows the Rows A \& B baseline sectional lift comparison with published data ${ }^{27}$.

The A \& B location was chosen because it is the most inboard location of pressure acquisition and most closesly represents the lift of a $2 \mathrm{D}$ wing. The values match relatively closely. It was expected that the lift would be lower than that of the 2D case although this is not the case. This source of error can come from a few sources. First, the pressure integration uses distinct points rather than continuous pressure data. This limits the accuracy of the lift calculation. There is also error in the accuracy of pressure scanner which factors into the calculation of the lift. Finally, the pressure integration assumes a perfect NACA 0015 wing. Realistically, inaccuracies in the manufacturing process make this not true. In general though, this plot shows that the section lift is in the range that would be expected for this wing.

Next, it was necessary to verify the the existence of the laminar separation bubble. Durring the testing process, spikes in $\mathrm{Cp}$ were seen between an $\mathrm{x} / \mathrm{c} 0.15$ and 0.5 . At first it was thought to be leaks in the pressure ports or a malfunction in the pressure scanner. After some more testing it 
was realized that the spike in $\mathrm{Cp}$ was a laminar separation bubble. The $\mathrm{Cp}$ data in this study is compared to that of another NACA 0015 study $^{28}$ in Figure 6.2.
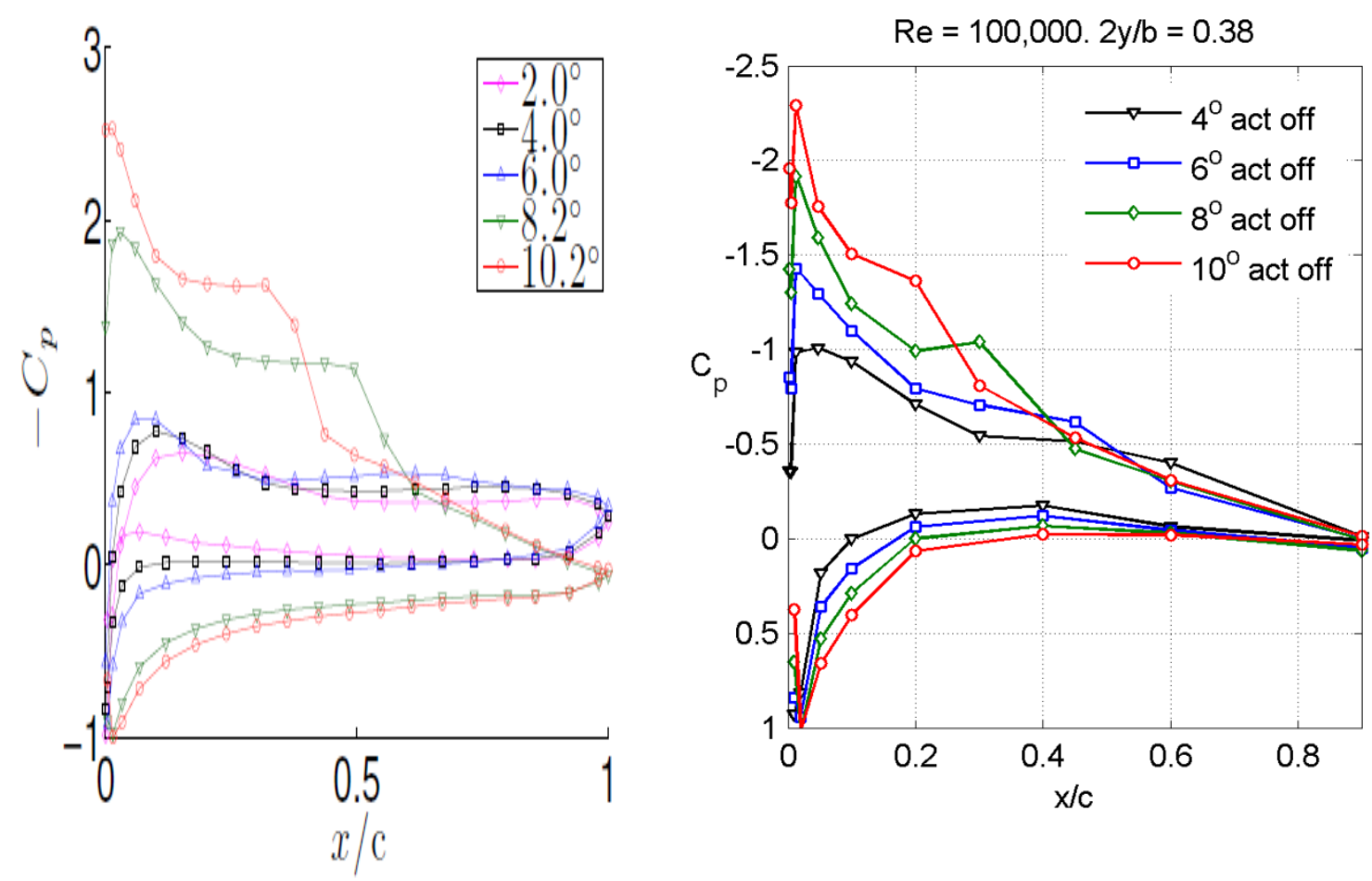

\section{Figure 6.2 Rows A \& B baseline Cp distribution at $\mathrm{Re}=100,000$ compared to NACA 0015 infinite wing ${ }^{28}$ at $\operatorname{Re}=60,000$}

The wing in the comparison study is $2-\mathrm{D}$ and was tested at a Reynolds number of 60,000 . Although these conditions are not ideal for comparison, this study was chosen due to the limited amount of data available. It can be seen that laminar separation bubbles occur in both cases. The higher peak $\mathrm{Cp}$ values in the comparison study can be attributed to the fact that the wing used in that study is infinite. An infinite wing has higher lift compared to that of a finite wing hence the higher $\mathrm{Cp}$ values near the leading edge. Also, the the difference in Reynolds number will change the location of the separation bubble but the goal of this chart is not to match Cp's exactly, but to show that the laminar separation bubble does indeed occur. 
An attempt at validating pressure data with XFOIL was made although it was found that XFOIL did not give an accurate pressure distribution for a NACA 0015 for Reynolds numbers used in this study. According to NACA Report $586^{27}$, at a Reynolds number of 100,000 and an angle of attack of 10 degrees, the sectional lift coefficient should be around 0.85 . XFOIL predicted a lift coefficient of 1.02. XFOIL also showed much more suction on the top surface of the wing than the results from this study, with a peak $\mathrm{Cp}$ of -3.65 . This was expected since the lift was also overpredicted. Therefore, XFOIL could not be used to validate the $\mathrm{Cp}$ data. Next, the variation in section lift along the y-axis was compared to published results in Figure 6.3

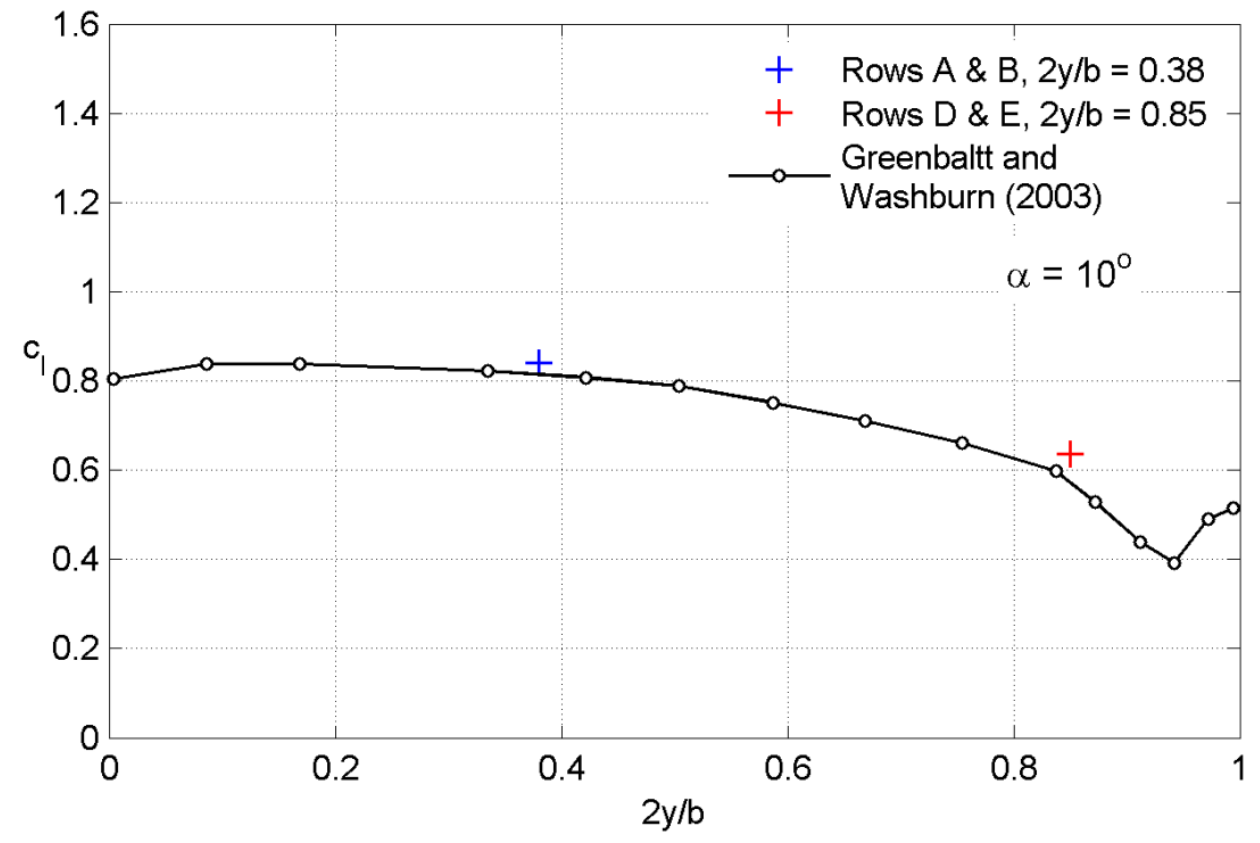

Figure 6.3 Baseline sectional lift compared to NACA 0015 non-tripped finite wing $(\operatorname{Re}=500,000 \mathrm{AR}=4)^{29}$ at $\alpha=10^{\circ}$

Figure 6.3 shows the baseline sectional lift comparison with a study by Greenblatt and Washburn $^{29}$ (2003). They report that the lift on a NACA 0015 wing is highest near the root and decreases until just before the tip. There is an increase in lift near the tip which is most likely attributed to the low pressure region caused by the tip vortex. The study slightly underpredicts the baseline data. This can be attributed to the difference in Reynolds number. Greenblatt and 
Washburn (1937) shows that there is slight variation in lift with Reynolds number for a NACA 0015 .

The lift and drag provided by the load cell needed to be validated. Figure 6.4 shows the total lift for the baseline case compared to that of a study by Marchman et $\mathrm{al}^{30}(1998)$.

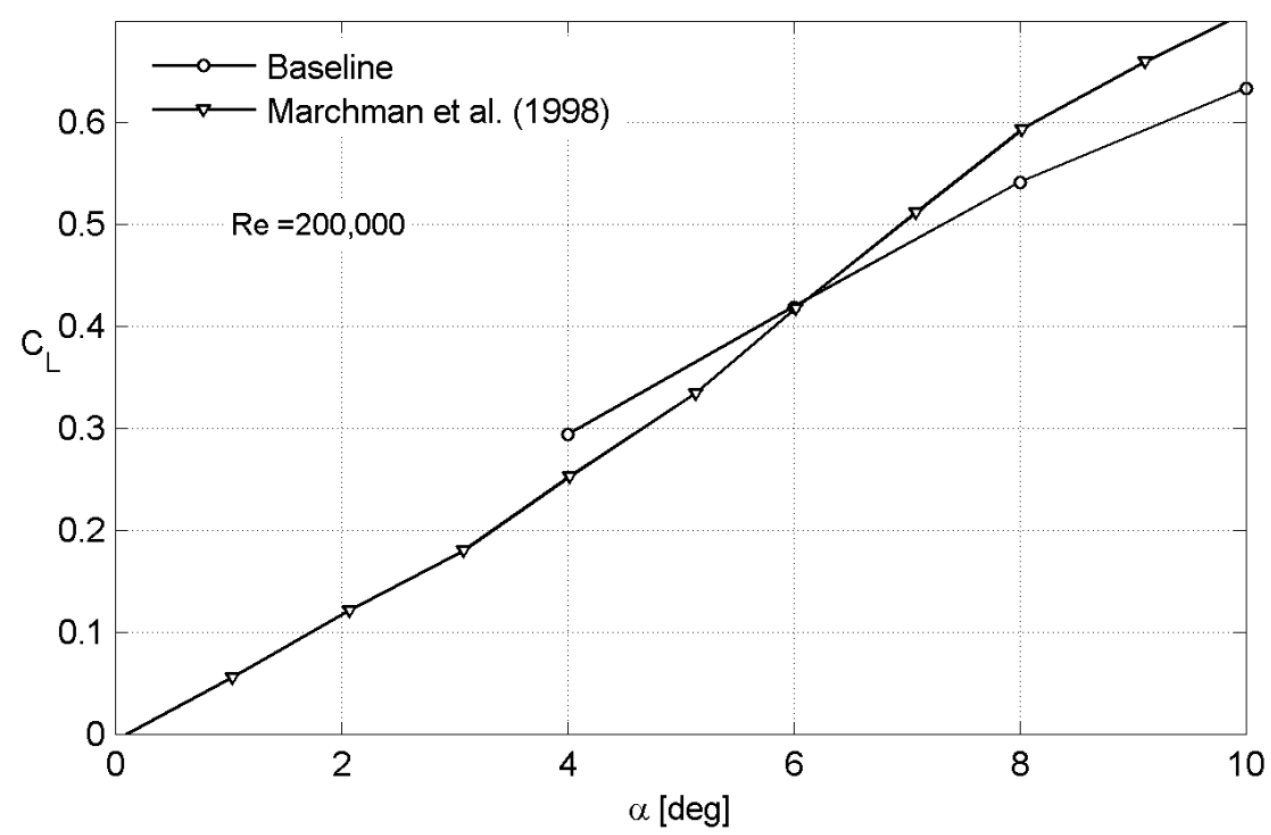

Figure 6.4 Baseline total lift at $\operatorname{Re}=200,000$ compared to NACA 0015 non-tripped finite wing $(\operatorname{Re}=200,000, A R=4)^{30}$

The baseline lift seems to fall in a similar range as that of the study by Marchman et al. (1998) . The lift slope seems to be slightly off than that form the study. This can be due to a combination of load cell accuracy and accuracy in the model geometry. This figure shows that the lift falls in a rang that would be expected of a wing of this type.

Validating the drag data was a difficult task because drag is highly dependent on angle of attack, Reynolds number, Mach number, surface finish, turbulent or laminar flow, aspect ratio, and many other factors. Many of these parameters are unique to test the particular model therefore, it is difficult to find data to validate drag. For this validation, several sources were 
found, as shown in Figure 6.4, to show that the value of drag falls into a range that is expected for a wing model of this type.

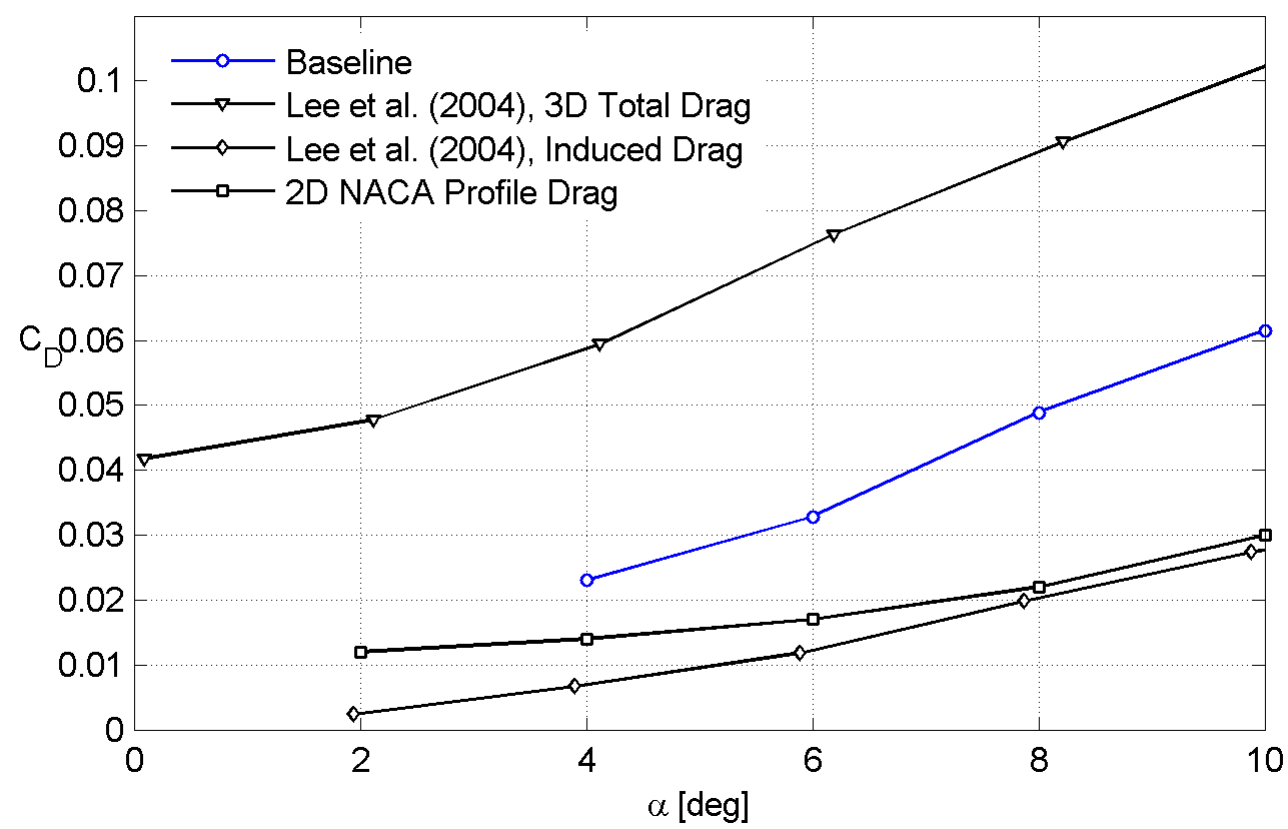

Figure 6.5 Baseline total drag compared against: 1) NACA 0015 finite wing total and induced drag at $\mathrm{Re}=200,000 \mathrm{AR}=3$ with transition strips ${ }^{31}$. 2) NACA 0015 data from NACA Report $586^{27}$

The first source of drag data is 3D total and induced drag data for a NACA 0015 wing with an aspect ratio of 3 , Reynolds number of 200,000 , and a tripped boundary layer ${ }^{31}$. The total drag from the study is higher than the drag obtained for the current study. This can be attributed to the lower aspect ratio and the transition strips. A lower aspect ratio wing has stronger tip effects which lead to an increased induced drag coefficient compared to a high aspect ratio wing. The transition strips create turbulent flow over the wing increasing the profile drag. It is reassuring that the total drag coefficient for the current model is lower than that of the tripped NACA 0015 but higher than the induced drag coefficient. The second source of drag data is $2 \mathrm{D}$ profile drag from a NACA report at a Reynolds number of 166,000 . The profile drag is lower than the drag obtained in this study which is expected because a 2D wing has no induced drag. This trend once again shows that the data collected in this study is what would be expected for a wing of this type. 


\subsection{Momentum Coefficient}

The first step in calculating the momentum coefficient was to get an idea of how the RMS velocity varies with frequency and amplitude. The hotwire was placed mid slit and acquisitions were taken. The frequency of the drive signal used to excite the actuators was increased by $10 \mathrm{hz}$ for each successive aquisition. A plot of frequency vs RMS velocity is shown in Figure 6.6.

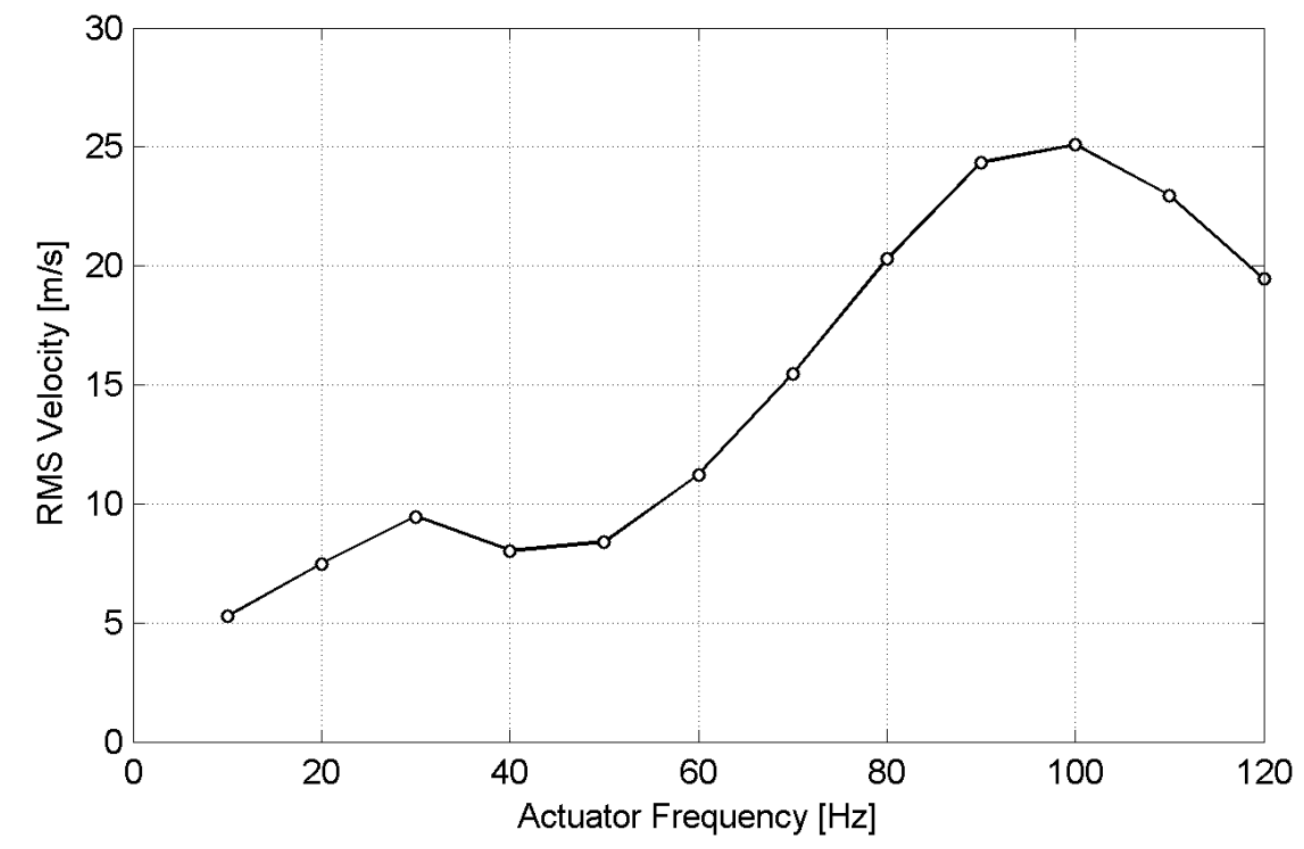

Figure 6.6 Synthetic jet RMS velocity for a range of frequencies

Interestingly, actuation at $100 \mathrm{~Hz}$ provides the highest RMS velocity. It was expected that the RMS velocity would be constant across the range of frequencies tested. The explanation for this occurrence was found by observing the jet instantaneous velocity profiles at each frequency, as seen in Figure 6.7 

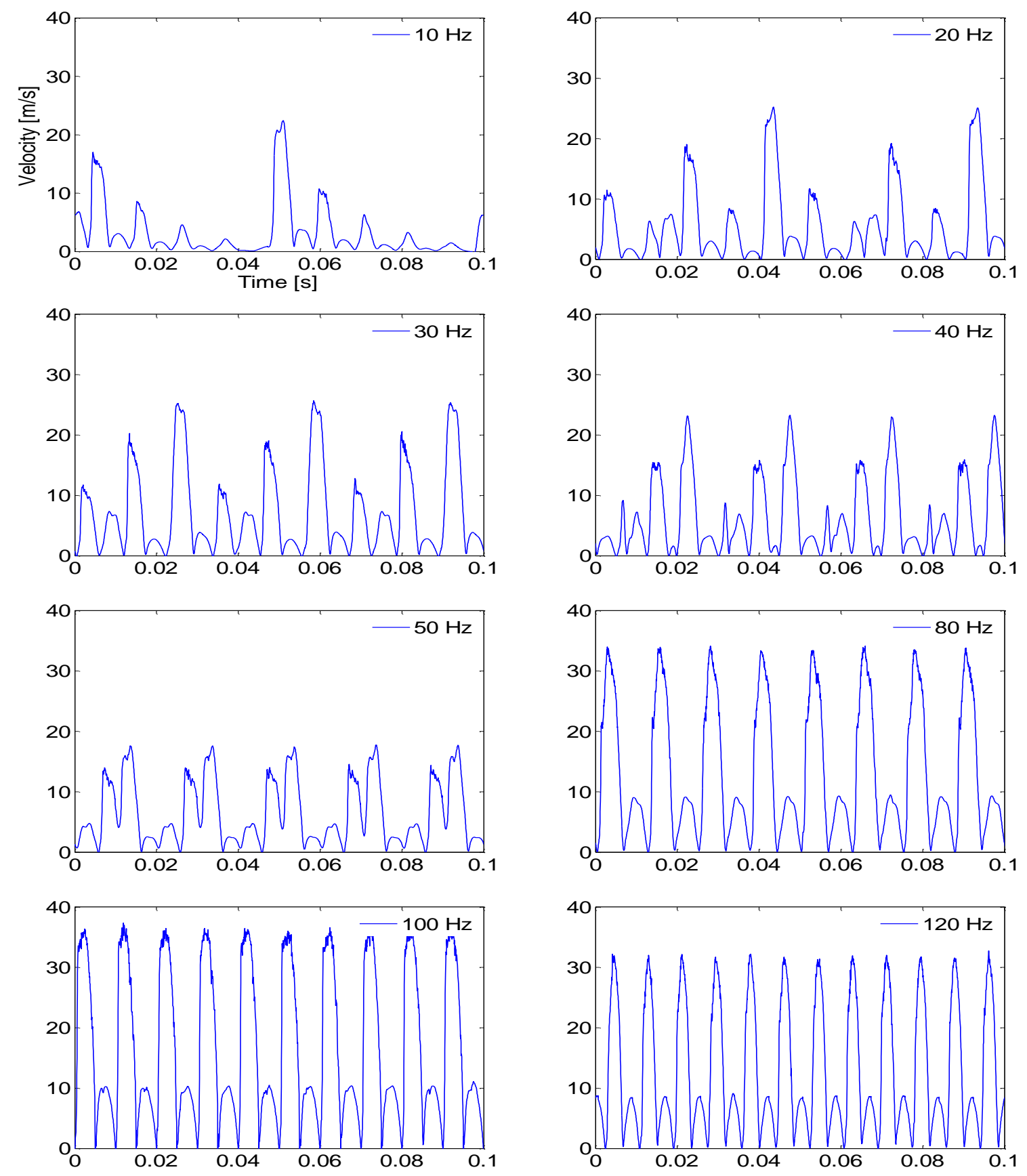

Figure 6.7 Synthetic jet instantaneous velocity profile

Several things can be observed by studying at the velocity profiles. The velocity profile is positive due to the rectifying nature of the hotwire. The wire can only sense the magnitude of the flow and cannot sense if the flow is going toward or away from the wire. Therefore, the wire cannot tell if the jet is ingesting or expelling fluid and shows the entire velocity profile as positive. The velocity profile also has distinct pattern. At $100 \mathrm{hz}$ there are large peaks followed 
by small peaks. The large peaks correspond to the out-stroke and the small peaks correspond to the in-stroke and have been seen similarly in other studies ${ }^{21}$. The ingestion peaks are smaller because of the ingetion flow patterns sensed by the hotwire. The hotwire is placed a slight distance above the slit to prevent damage to the hotwire filament. Any small movement can bump the hotwire into the side wall of the slit and break the wire. The hotwire must be placed a small distance above the slit to minimize this risk. Because of the non-symmetry, the signal could not be used to calculate the momentum coefficient. The signal had to be rectified based on the outstroke signal, as shown in Figure 6.8, so the RMS velocity and momentum coefficient could be calculated.

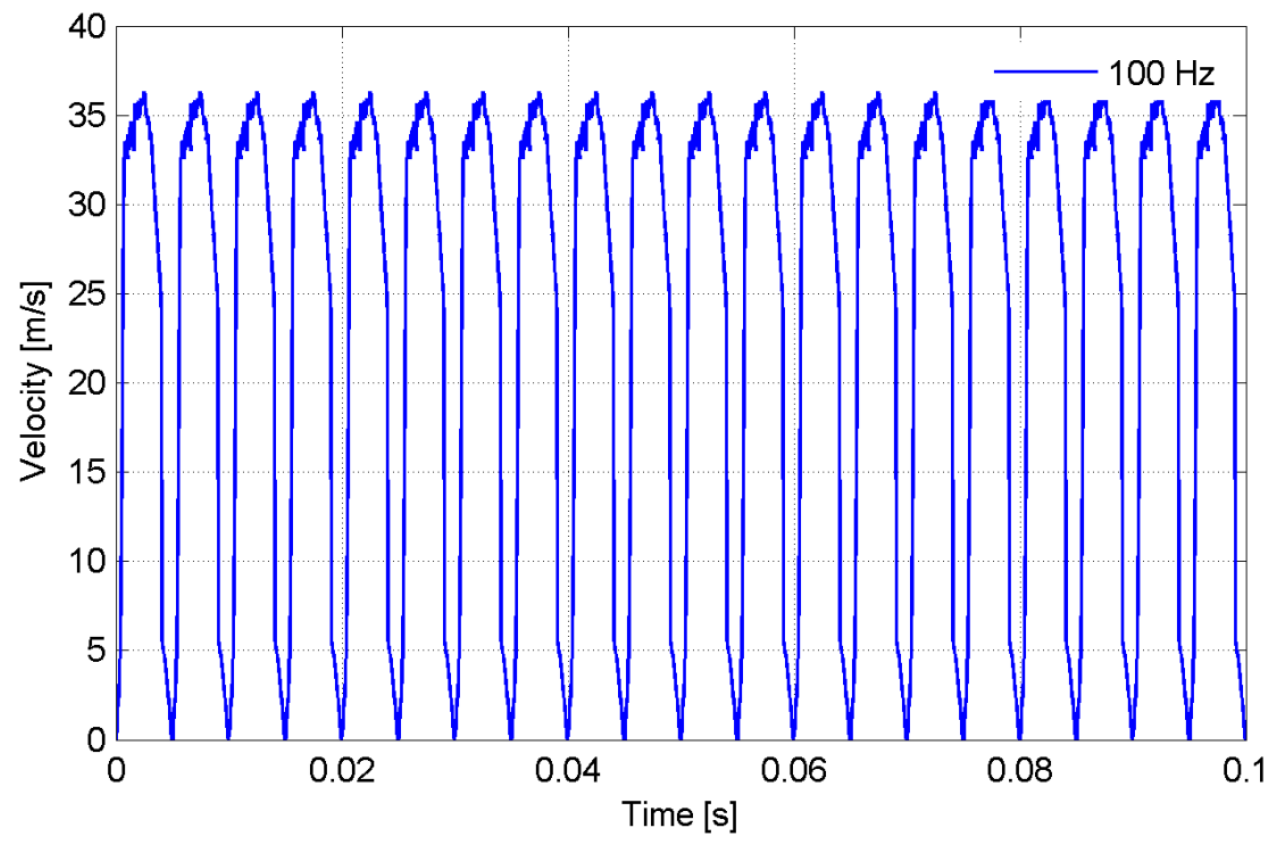

Figure 6.8 Rectifed hotwire signal

The final notable observation to be made from Figure 6.7 is the fluidic interference within the jet at low frequencies. This can be explained by the fact that the synthetic jet is created by the displacement of 8 different membranes. A pressure fluctuation is created by each disk and travels outwards at the speed of sound. Since each disk is a different distance from the exit of the jet, the time it takes for the pressure fluctuation to reach the jet exit is also different for each disk. This 
along with the fact that pressure fluctuations of individual disks can interfere with each other is a probable explanation of this occurrence. Further study into this fluidic interference would be interesting although it is not the goal of this Thesis.

The next test was performed to look at the velocity profile across the slit width. The results of this test are shown in Figure 6.9. The slit width begins at the side near the suction surface at 0.00 in and ends at 0.04 in (the slit is 0.04 in wide).

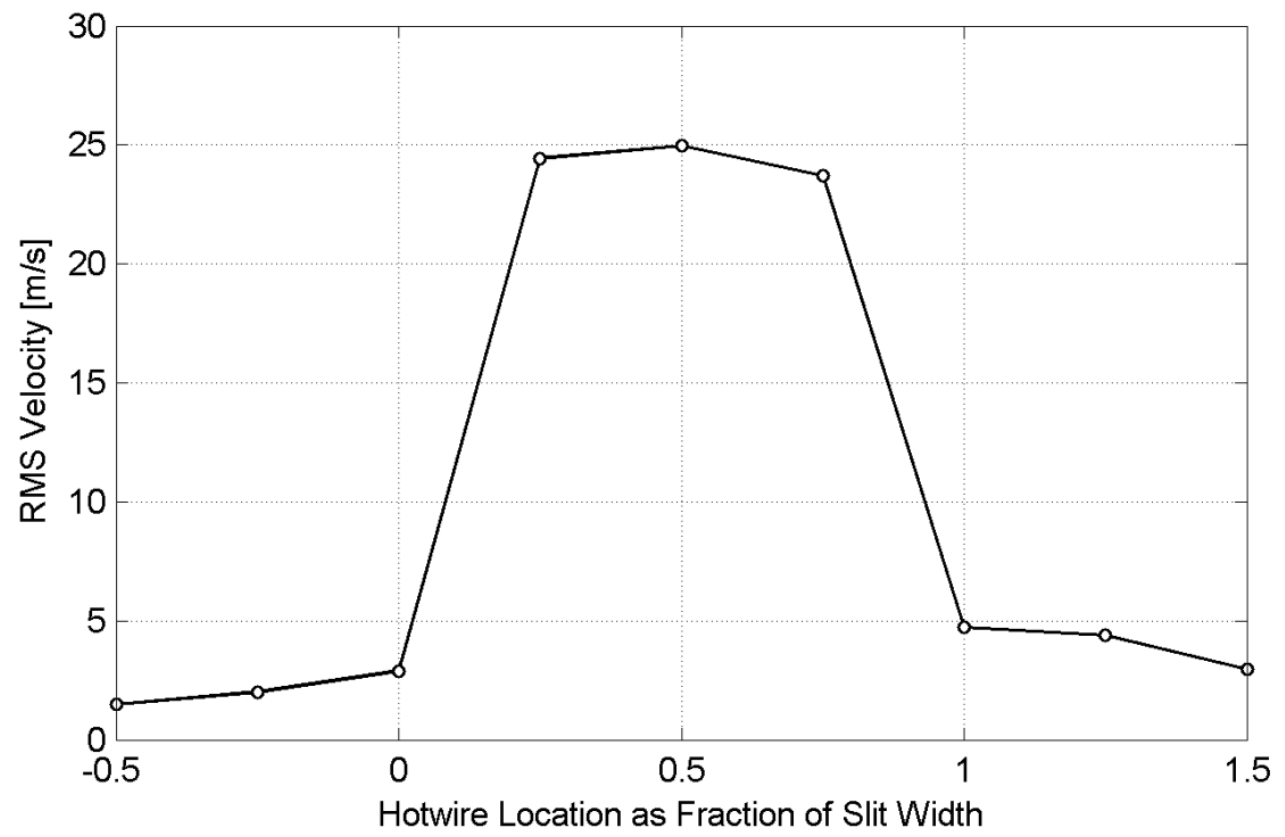

Figure 6.9 Jet velocity across 0.04 in. slit width

The RMS velocity profile along the slit width is constant and drops significantly outside the jet. It seems there is some recirculation at outside the edges of the slit since the RMS velocity does not go to zero. Next, the velocity profile was measured across 11 equally spaced locations along the length of the slit. The results are shown in Figure 6.10 


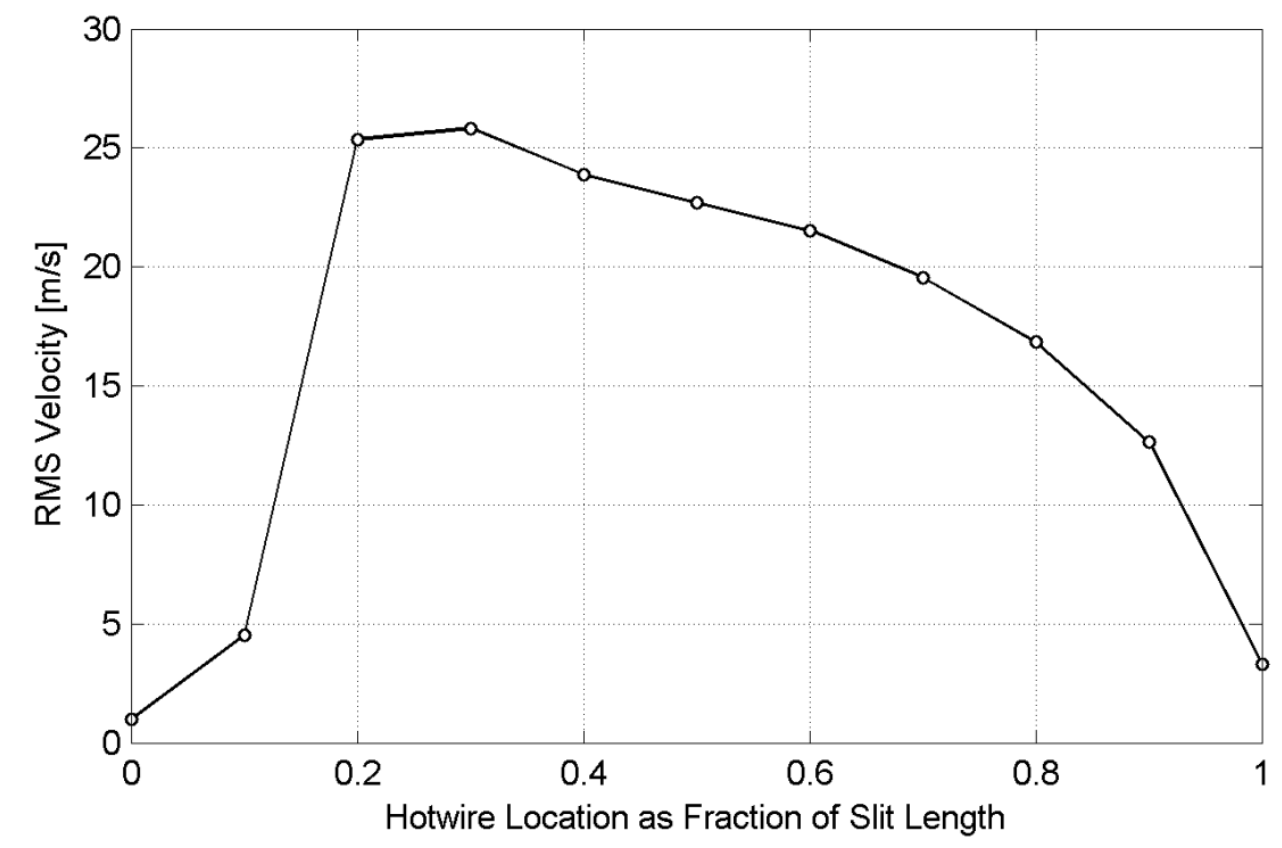

Figure 6.10 Synthetic jet velocity across slit length

The results show that the RMS velocity is not constant along the slit length. For this experiment, this is fine because the RMS velocity can be integrated across the slit length to get $\mathrm{C}_{\mu}$. The results of the amplitude test are shown in Figure 6.11

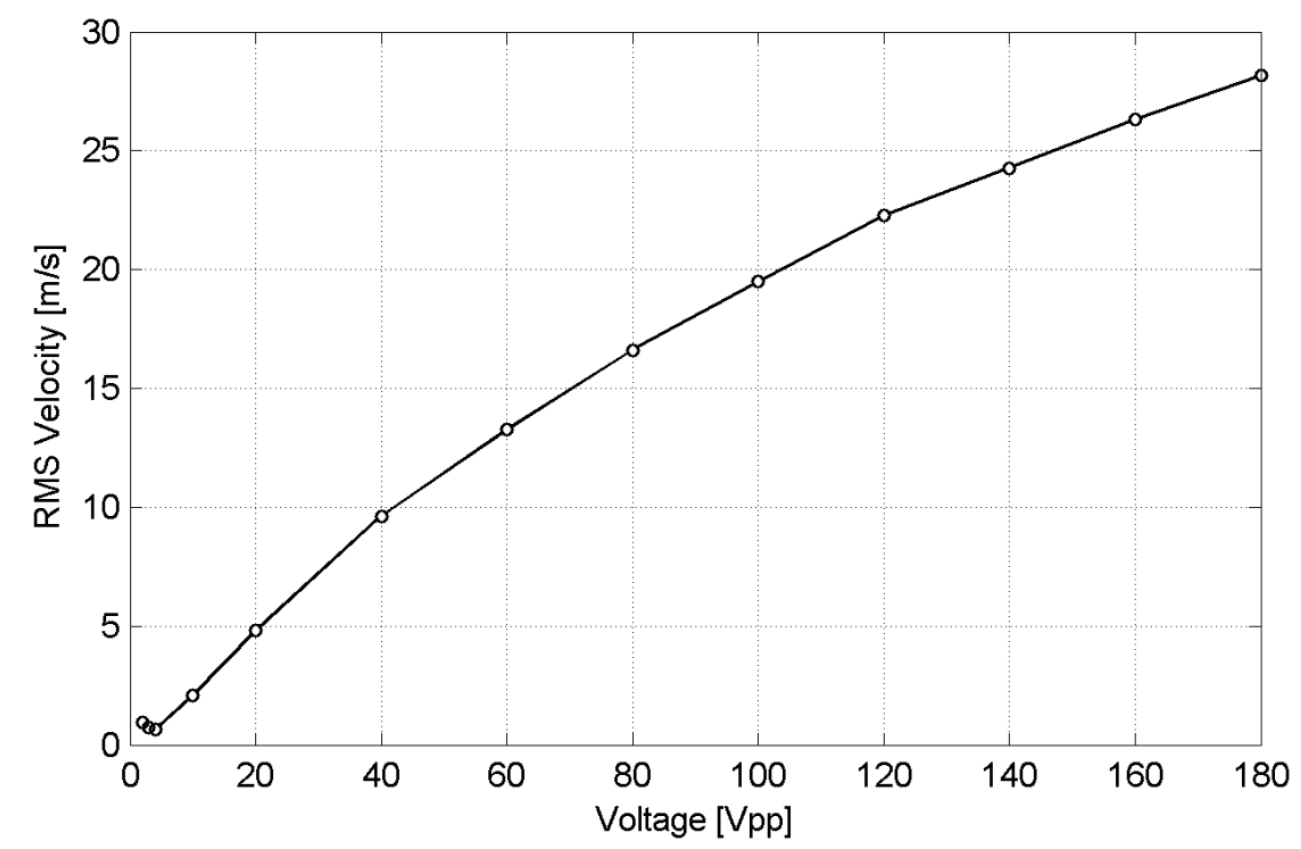

Figure 6.11 Change in RMS velocity with piezoelectric disk driving signal amplitude 
These results were as expected. The RMS velocity goes to zero when the voltage is zero and starts to increase as voltage is increased. The convex nature of the plot shows that the RMS would reach an asymptote eventually as the voltage is increased although these disks are not rated for more than $180 \mathrm{Vpp}$ so the voltage was not increased further. With these three plots the momentum coefficient could be calculated. These tests were done so the momentum coefficient could be calculated at various frequencies but due to fluidic interference between the 8 piezoelectric disks only frequencies near $100 \mathrm{~Hz}$ worked well. Testing was done a $100 \mathrm{~Hz}$ independent of Reynolds number. $\mathrm{F}^{+}$was allowed to vary because a previous study showed little change in effect due to $\mathrm{F}^{+12}$. A plot of $\mathrm{C}_{\mu}$ for various Reynolds numbers is shown in Figure 6.12.

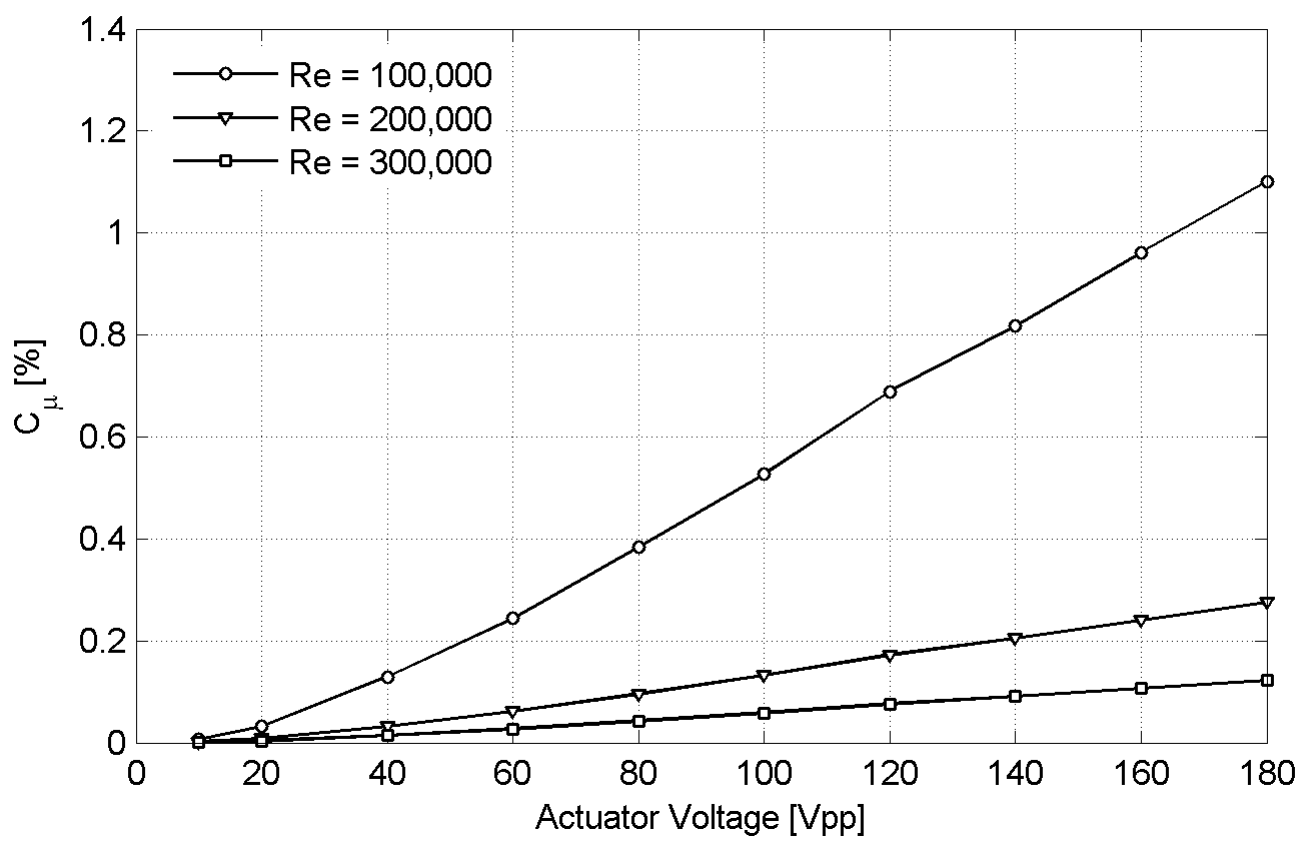

Figure 6.12 Momentum coefficient expected at various Reynolds numbers

The results show the momentum coefficient plotted for three Reynolds numbers. $\mathrm{C}_{\mu}$ decreases with Reynolds number and increases with driving signal voltage. $C_{\mu}$ is significantly higher for $\operatorname{Re}=100,000$. The results of the plot are tabulated in Table 6.1 for reference in later sections. 
Table 6.1 Tabulated $C_{\mu}$ values for $f=100 ~ H z$

\begin{tabular}{|c|c|c|c|}
\hline \multirow{2}{*}{$\begin{array}{c}\text { Voltage } \\
(\text { Vpp })\end{array}$} & \multicolumn{3}{|c|}{ Momentum Coefficient } \\
\cline { 2 - 4 } & $\mathrm{Re}=100,000$ & $\mathrm{Re}=200,000$ & $\mathrm{Re}=300,000$ \\
\hline 10 & $0.01 \%$ & $0.00 \%$ & $0.00 \%$ \\
\hline 20 & $0.03 \%$ & $0.01 \%$ & $0.00 \%$ \\
\hline 40 & $0.13 \%$ & $0.03 \%$ & $0.01 \%$ \\
\hline 60 & $0.24 \%$ & $0.06 \%$ & $0.03 \%$ \\
\hline 80 & $0.38 \%$ & $0.10 \%$ & $0.04 \%$ \\
\hline 100 & $0.53 \%$ & $0.13 \%$ & $0.06 \%$ \\
\hline 120 & $0.69 \%$ & $0.17 \%$ & $0.08 \%$ \\
\hline 140 & $0.82 \%$ & $0.20 \%$ & $0.09 \%$ \\
\hline 160 & $0.96 \%$ & $0.24 \%$ & $0.011 \%$ \\
\hline 180 & $1.10 \%$ & $0.28 \%$ & $0.12 \%$ \\
\hline
\end{tabular}

\subsection{Pressure Measurement Results}

This section discusses the results obtained from the pressure data collected by the RAD 3200 system. The goal of these results was to see how the sectional lift coefficient changes with actuation. Error bars were omitted from these results to avoid clutter. Appendix B shows the error analysis and has sample plots with error bars. The sectional lift coefficients for the baseline case and the actuated case are shown in Figure 6.13. 

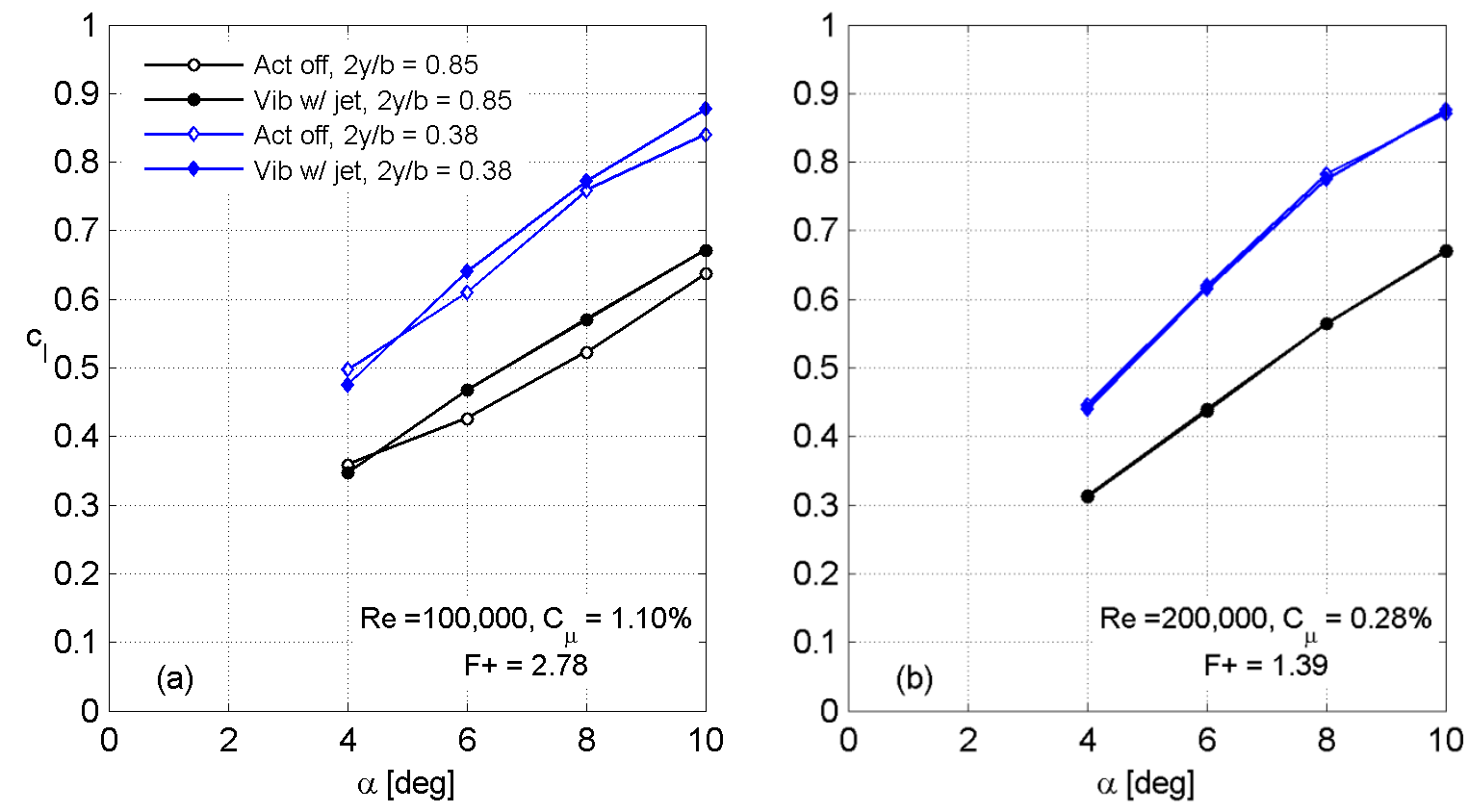

Figure 6.13 Sectional lift coefficient comparison for baseline and actuated cases

The trends in the $\operatorname{Re}=100,000$ case in Figure 6.13 seemed strange because it was expected that a jet coming out of the wingtip would have significantly more effect on $c_{1}$ near the wingtip. It will be later shown why the effect was less significant than expected. These results show that actuation has a large effect at both $2 \mathrm{y} / \mathrm{b}=0.38$ and $2 \mathrm{y} / \mathrm{b}=0.85$ locations instead of just the most outboard location at $2 \mathrm{y} / \mathrm{b}=0.85$. During the test, structural vibrations could be felt by touching the wing. It was predicted that vibration has some effects on these results. The effect of vibration was isolated from the effect of the synthetic jet by using tape and a relief valve, as discussed in Section 5.6, to eliminate the synthetic jet while keeping the wing vibrating. The results showing the effect of vibration are shown in Figure 6.14. 

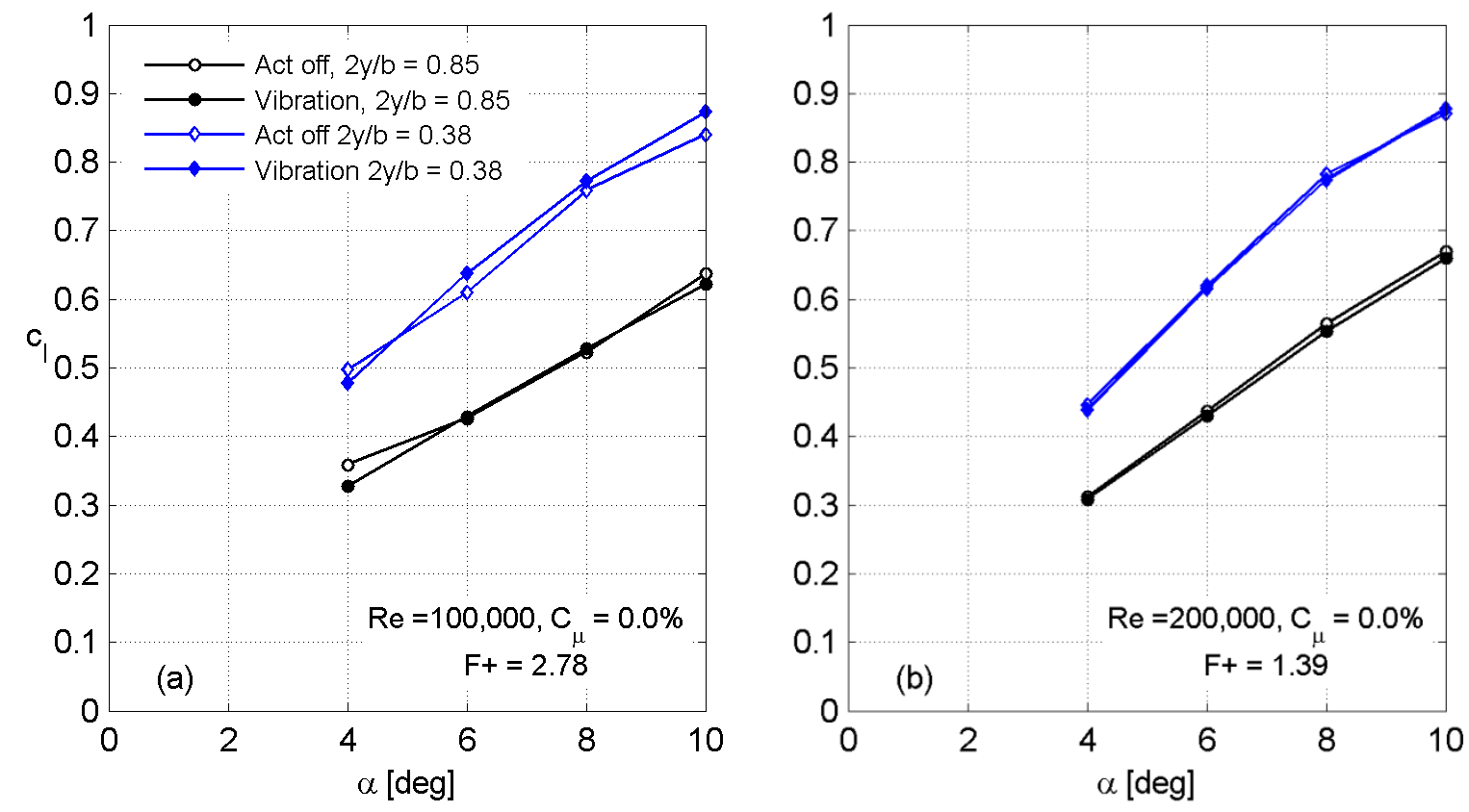

Figure 6.14 Sectional lift coefficient comparison isolating the effect of vibration

Figure 6.14 shows that vibration had an effect at $R e=100,000$ regardless of the $2 \mathrm{y} / \mathrm{b}$ location but it is difficult to see any trends from this data except that vibration had less effect at $\operatorname{Re}=200,000$. This could explain the reason the previous plot showed effects for both $2 \mathrm{y} / \mathrm{b}$ locations. The effect of the synthetic jet was isolated in Figure 6.15 

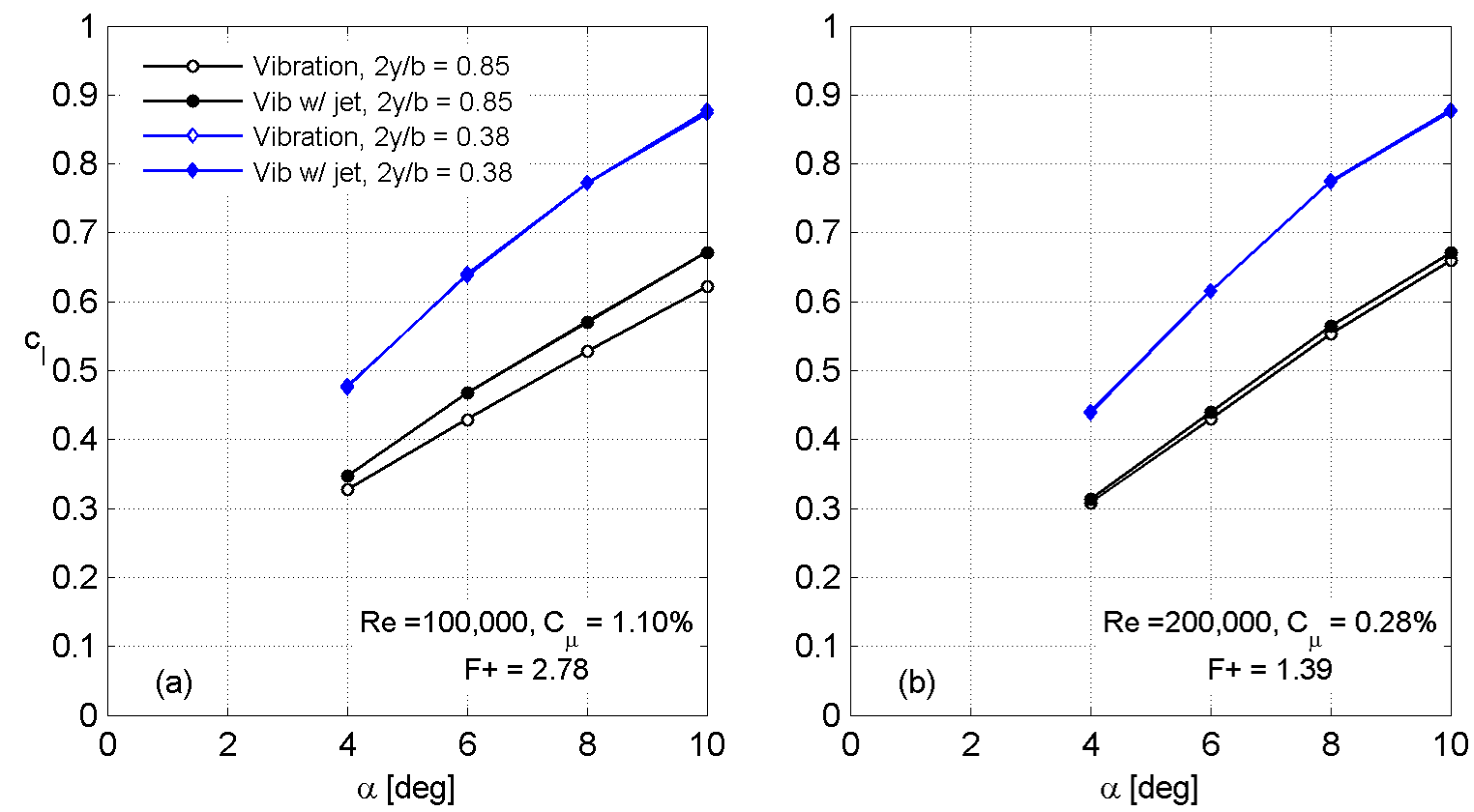

Figure 6.15 Sectional lift coefficient comparison isolating the effect of the synthetic jet

These results show the expected result from the synthetic jet. The synthetic jet showed significantly more effect near the wingtip. Rows D \& E at $2 \mathrm{y} / \mathrm{b}=0.85$, which are much closer to the wingtip, show an increase in the sectional lift coefficient with the jet present and Rows A \& B at $2 \mathrm{y} / \mathrm{b}=0.38$ do not. At $\operatorname{Re}=200,000$ there is less effect which can be attributed to the lowered momentum coefficient, although the lift is still slightly increased towards the tip.

The cause of the sectional lift coefficient trends was investigated by looking into the $\mathrm{Cp}$ distributions. For the following results, there were no noticeable differences in the pressure distribution on the pressure surface of the wing due to actuation. Because of this, the $\mathrm{Cp}$ distribution on the pressure surface was omitted to draw more attention to the suction surface. Appendix B.1. shows a sample plot with the Cp distribution on the pressure surface for reference. The effect of vibration on the Cp can be seen by looking at Figure 6.16 through Figure 6.19 . 


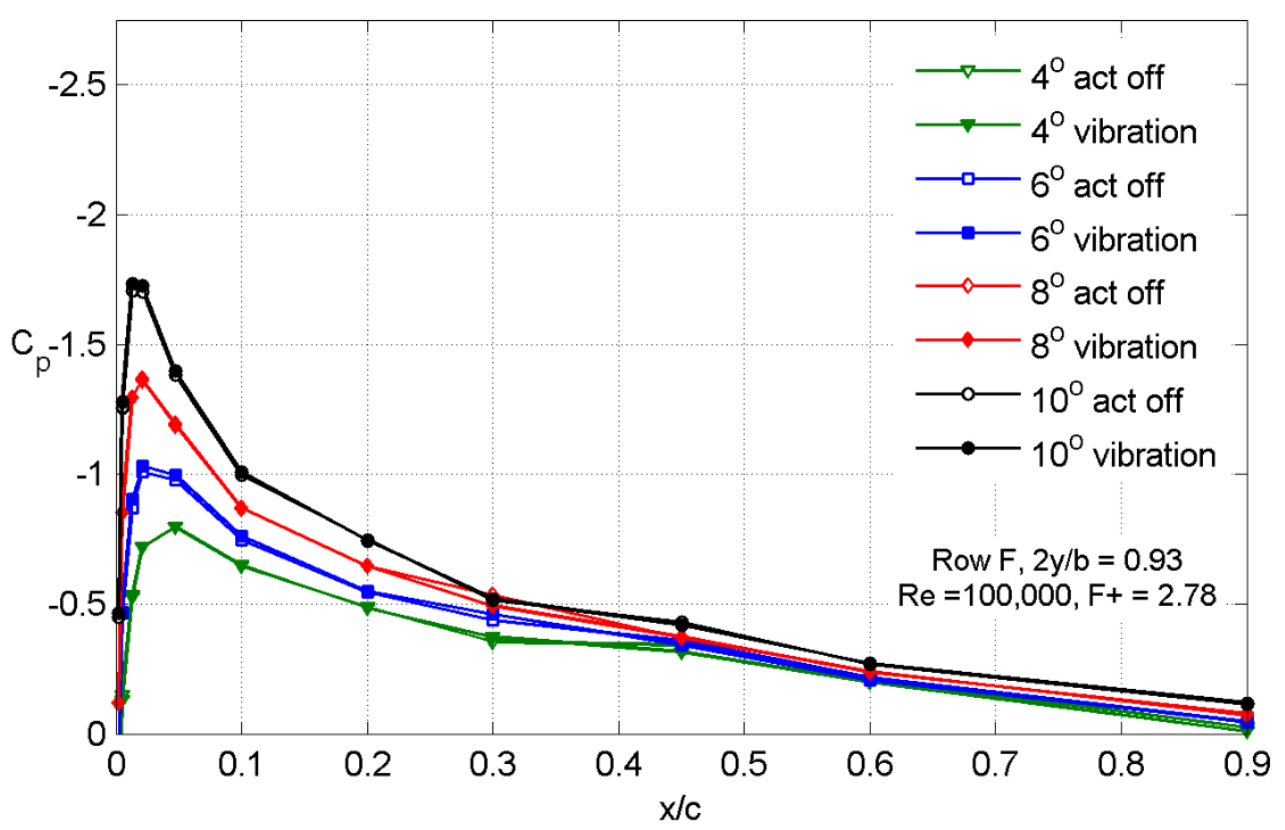

Figure 6.16 Row $F$ suction surface $C p$ distribution at $2 \mathrm{y} / \mathrm{b}=0.93$ showing effect of wing vibration at $F^{+}=2.78, C_{\mu}=0.00$, and $R e=100,000$

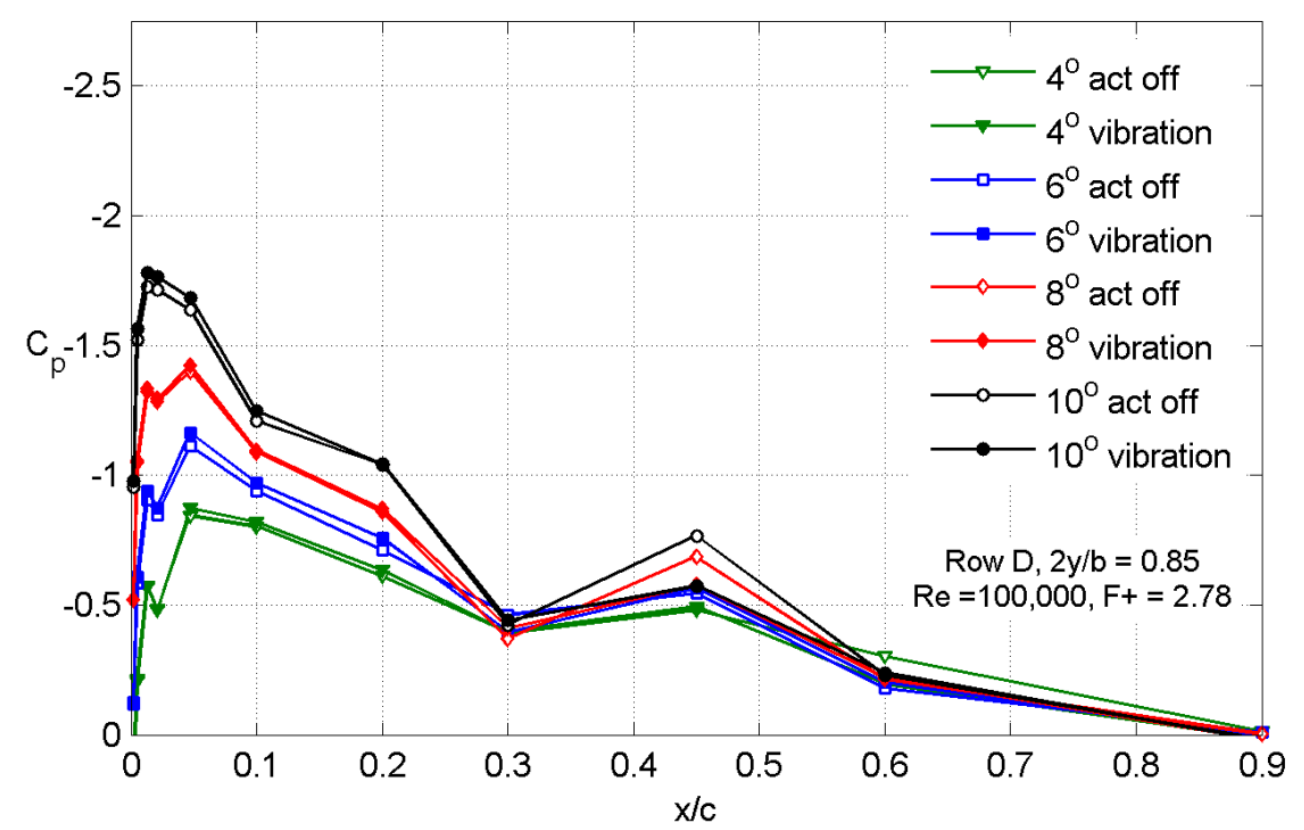

Figure 6.17 Row $D$ suction surface $C p$ distribution at $2 \mathrm{y} / \mathrm{b}=0.85$ showing effect of wing vibrationat at $F^{+}=2.78, C_{\mu}=0.00$, and $R e=100,000$ 


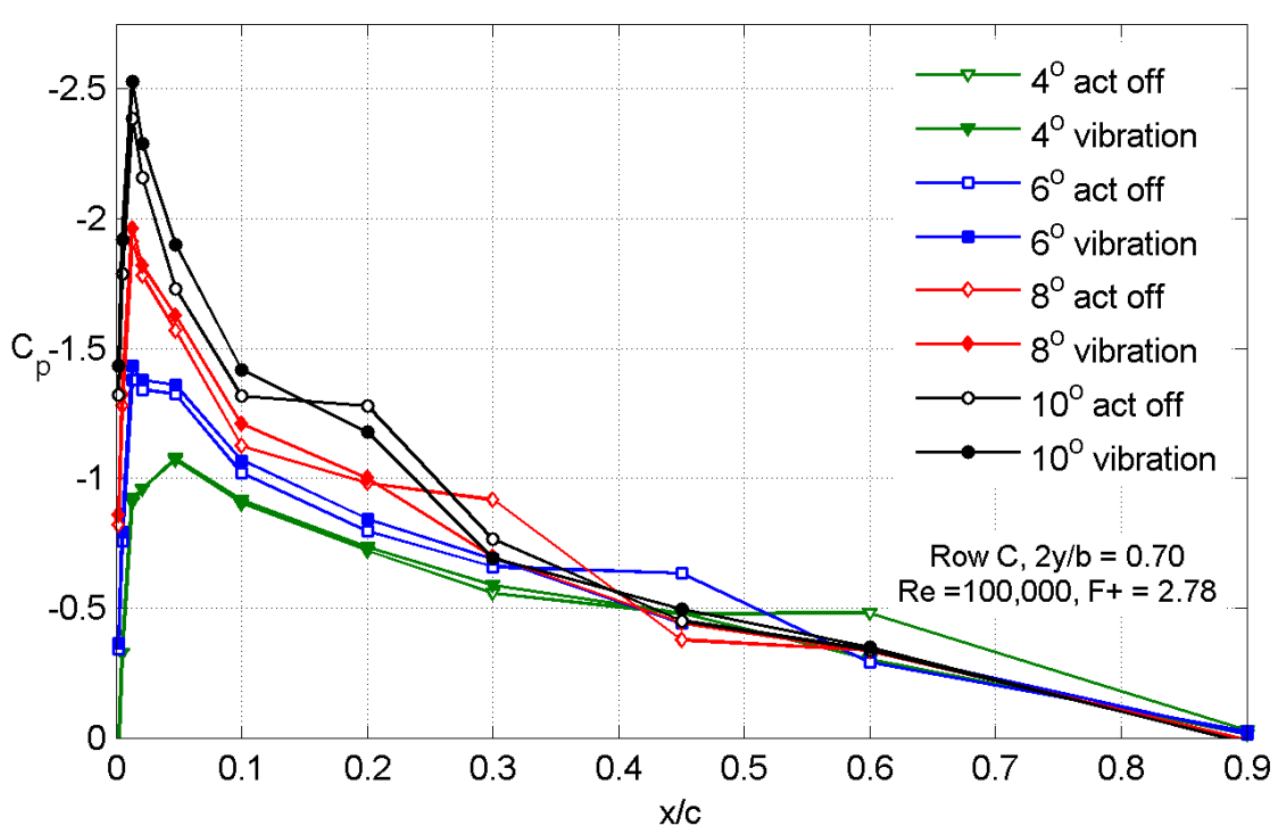

Figure 6.18 Row $C$ suction surface $C p$ distribution at $2 \mathrm{y} / \mathrm{b}=0.70$ showing effect of wing vibration at at $\mathrm{F}^{+}=\mathbf{2 . 7 8}, \mathrm{C}_{\mu}=\mathbf{0 . 0 0}$, and $\mathrm{Re}=\mathbf{1 0 0 , 0 0 0}$

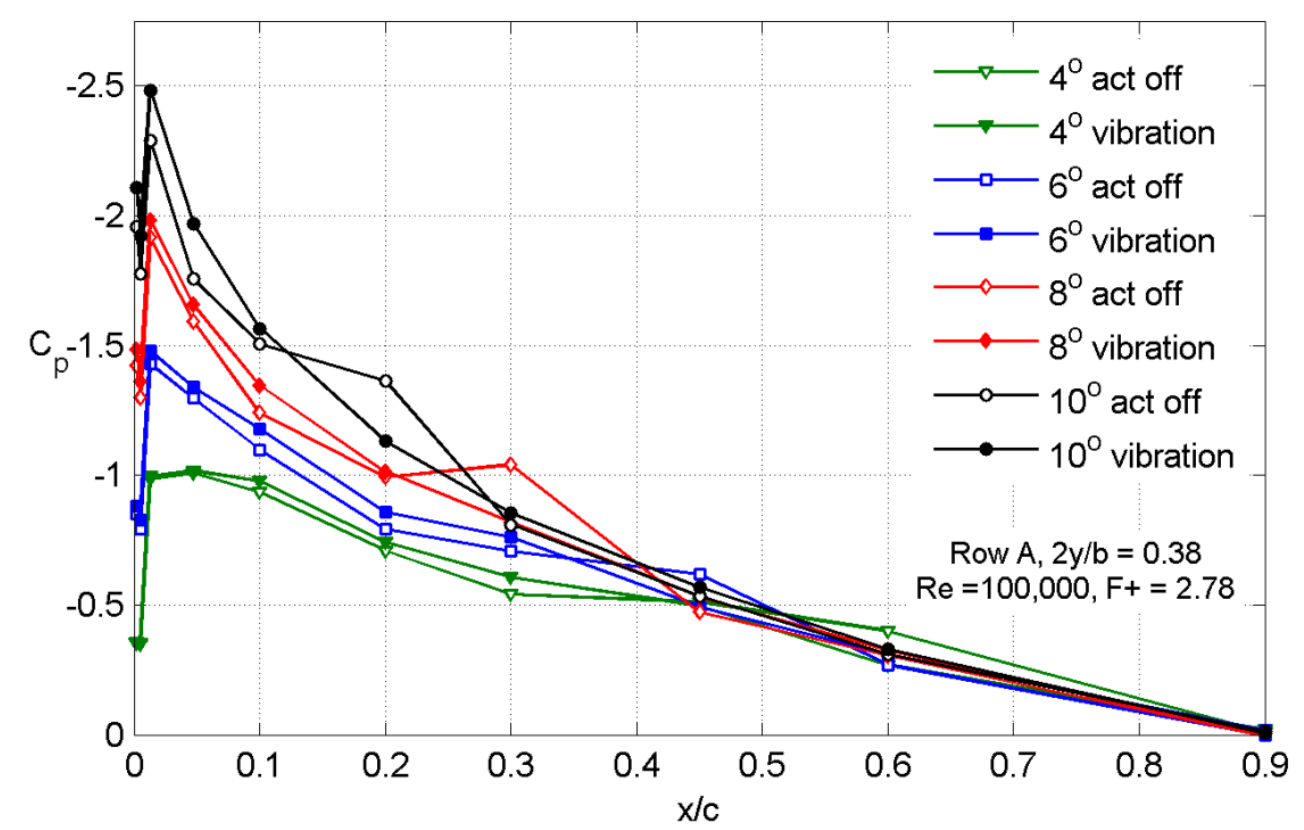

Figure 6.19 Row A suction surface $C p$ distribution at $2 \mathrm{y} / \mathrm{b}=0.38$ showing effect of wing vibration at at $\mathrm{F}^{+}=2.78, \mathrm{C}_{\mu}=0.00$, and $\mathrm{Re}=100,000$ 
Pressure irregularities indicative of laminar separation bubbles can be seen in Figure 6.16Figure 6.19. The laminar separation bubbles become more significant near the root. This is a well-observed phenomenon for low Reynolds number flows ${ }^{28}$. Vibration eliminates this separation bubble and increases the $\mathrm{Cp}$ distribution near the leading edge. At higher Reynolds number this bubble is much less prevalent. Figure 6.20 shows the $\mathrm{Cp}$ distribution for Row A at $2 \mathrm{y} / \mathrm{b}=0.38$ at $\operatorname{Re}=200,000$. There is no effect near the leading edge although vibration does have some effect after $\mathrm{x} / \mathrm{c}=0.3$. What can be concluded from these charts is that vibration eliminates the laminar separation bubble.

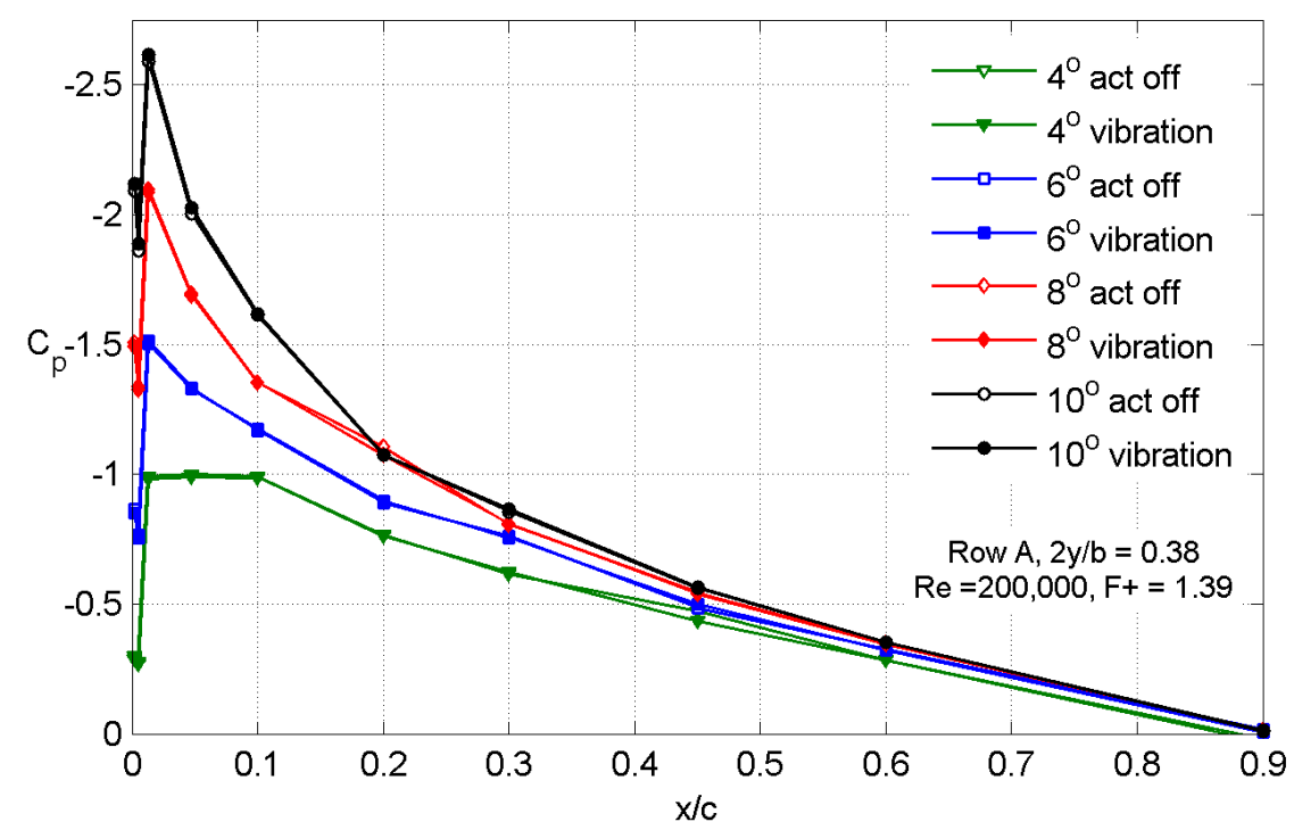

Figure 6.20 Row A suction surface $\mathrm{Cp}$ distribution at $2 \mathrm{y} / \mathrm{b}=0.38$ showing effect of wing vibration at at $\mathrm{F}^{+}=1.39, \mathrm{C}_{\mu}=0.00$, and $\mathrm{Re}=200,000$

Next the effect of the synthetic jet was isolated. Figure 6.21 through Figure 6.24 show the effect of the synthetic jet. 


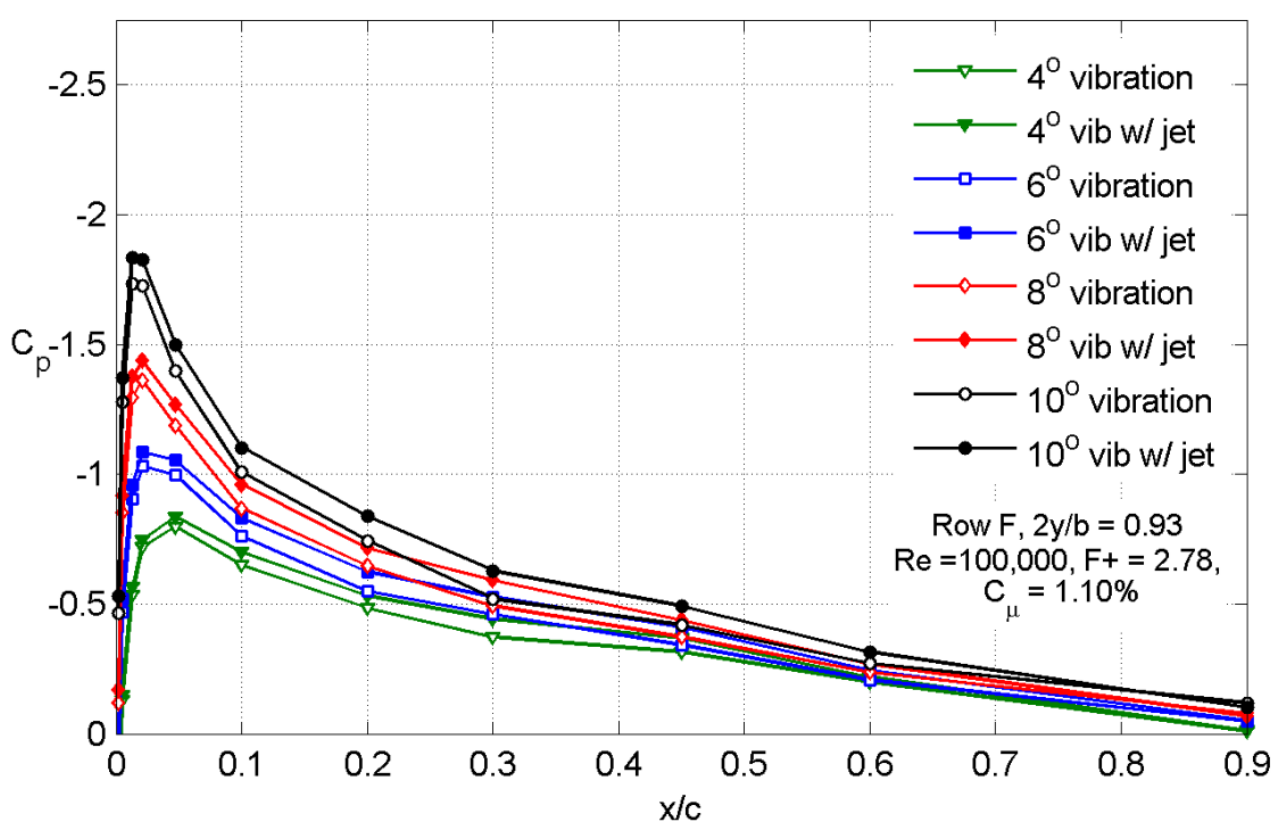

Figure 6.21 Row $F$ suction surface $C p$ distribution at $2 \mathrm{y} / \mathrm{b}=0.93$ showing effect of wing the synthetic jet at at $F^{+}=2.78, C_{\mu}=1.10 \%$, and $R e=100,000$

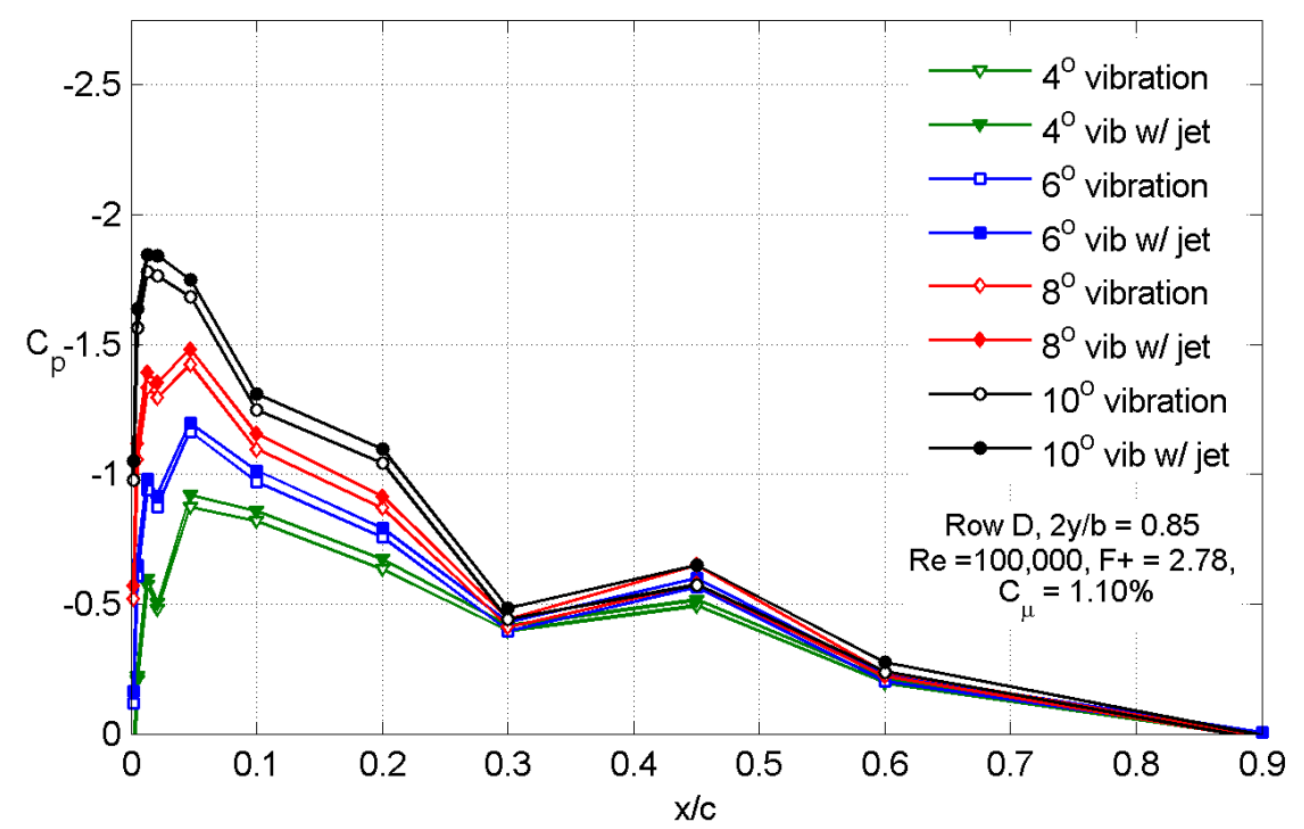

Figure 6.22 Row $D$ suction surface $C p$ distribution at $2 \mathrm{y} / \mathrm{b}=0.85$ showing effect of wing the synthetic jet at at $F^{+}=2.78, C_{\mu}=1.10 \%$, and $R e=100,000$ 


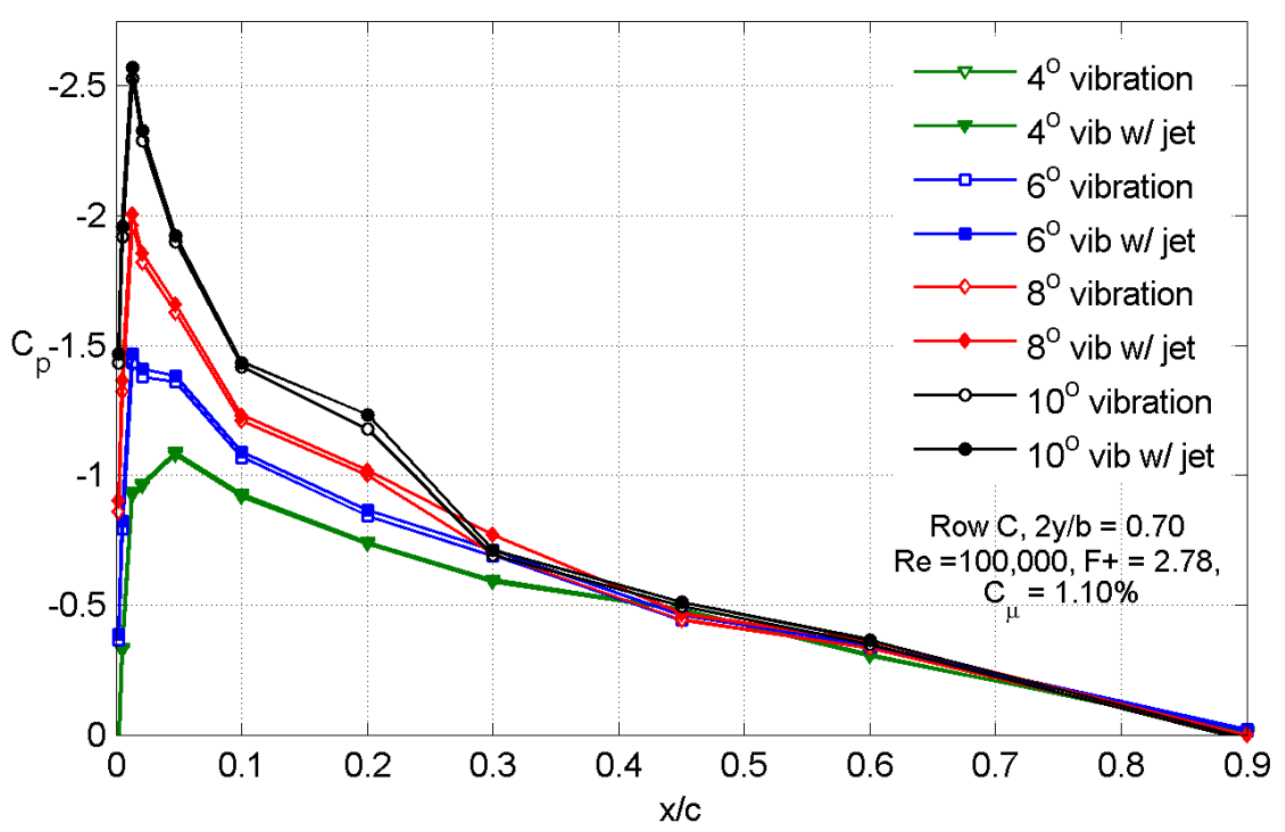

Figure 6.23 Row $C$ suction surface $C p$ distribution at $2 \mathrm{y} / \mathrm{b}=0.70$ showing effect of wing the synthetic jet at at $F^{+}=2.78, C_{\mu}=1.10 \%$, and $R e=100,000$

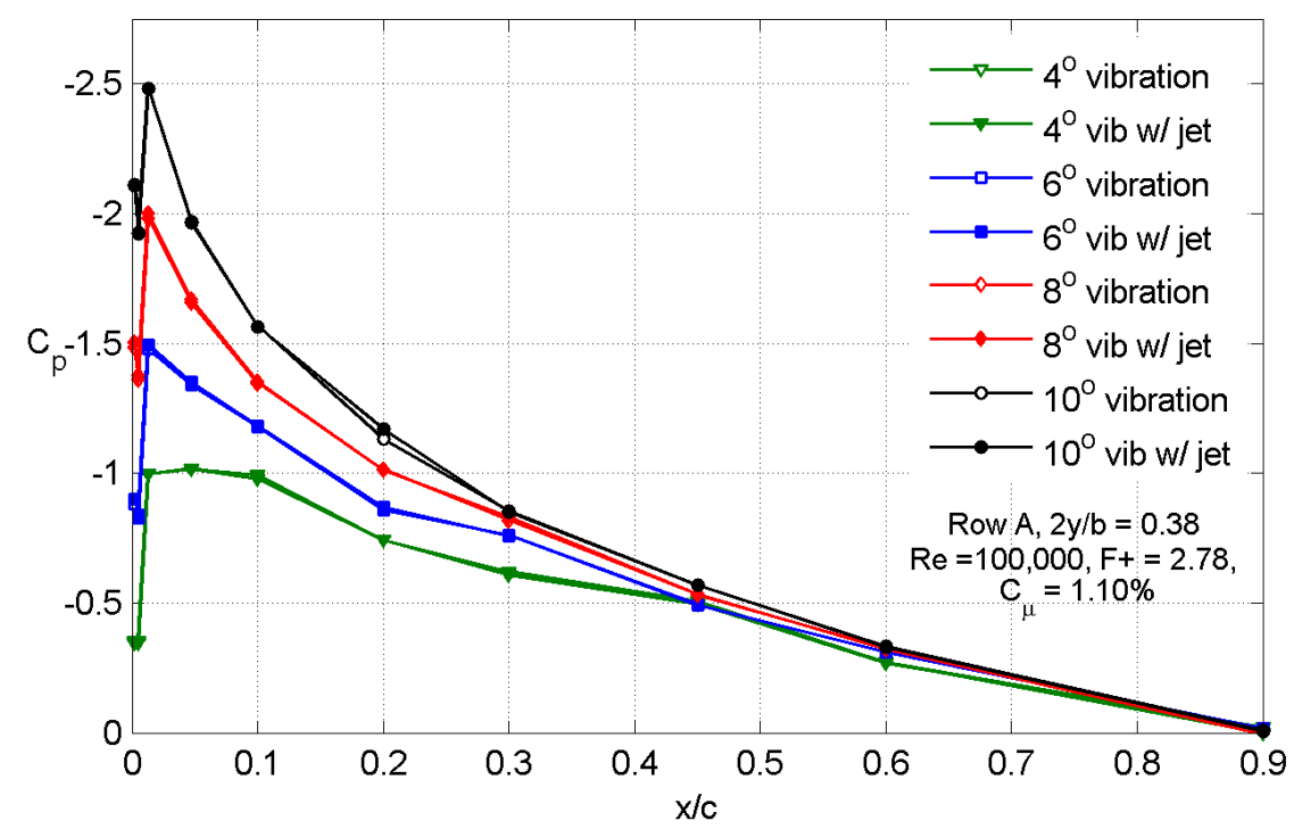

Figure 6.24 Row A suction surface $C p$ distribution at $2 \mathrm{y} / \mathrm{b}=0.38$ showing effect of wing thesynthetic jet at at $F^{+}=2.78, C_{\mu}=1.10 \%$, and $R e=100,000$

These results were expected from the synthetic jet. The jet increases the suction near the leading edge to about mid-chord. The effect becomes more significant at $2 \mathrm{y} / \mathrm{b}=0.70,0.85$ and 
0.93 closer to the tip since this is where the jet is located. The effect is also larger for higher angles of attack. This matches what was seen in the sectional lift coefficient results since $c_{1}$ increased more for higher angles of attack. Figure 6.25 shows the results for $\operatorname{Re}=200,000$.

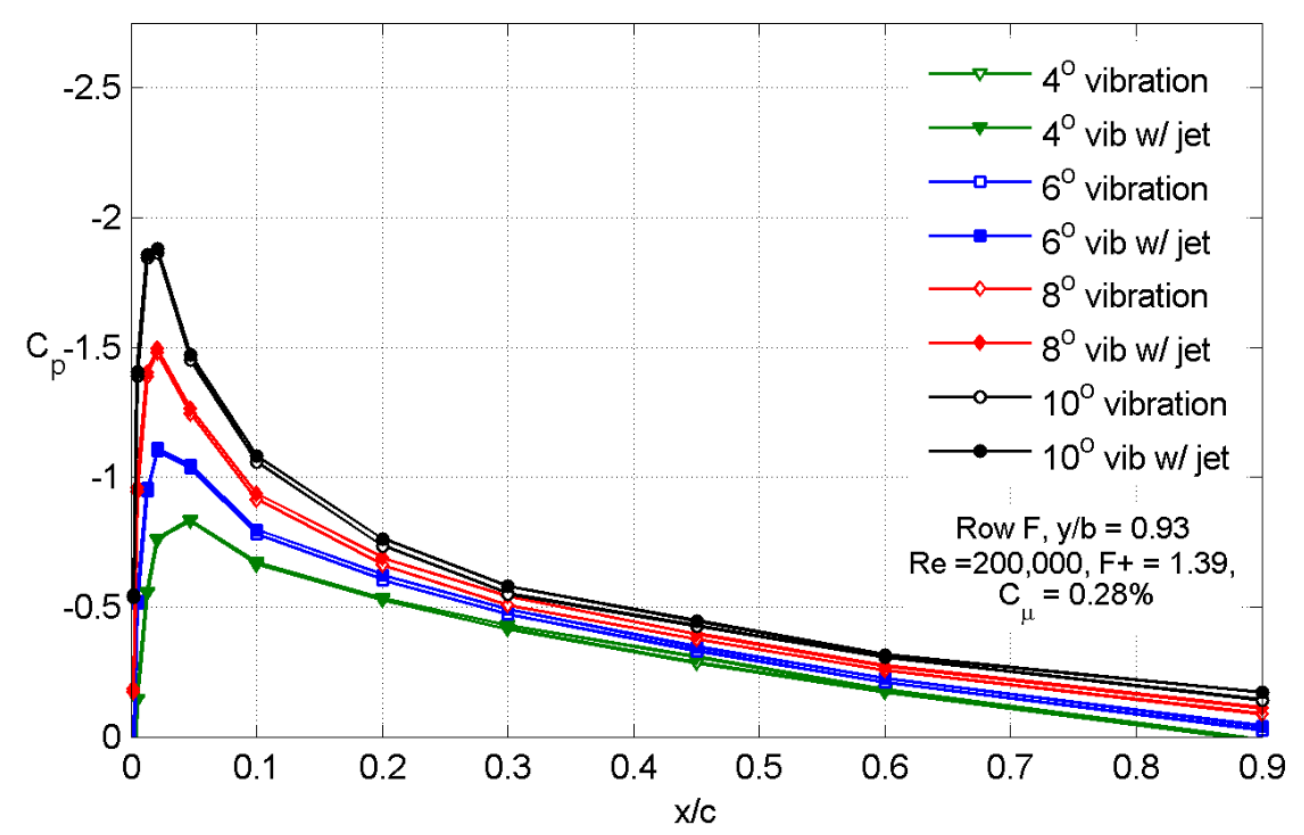

Figure 6.25 Row $F$ suction surface $C p$ distribution at $2 \mathrm{y} / \mathrm{b}=0.93$ showing effect of wing the synthetic jet at at $\mathrm{F}^{+}=1.39, \mathrm{C}_{\mu}=0.28 \%$, and $\mathrm{Re}=\mathbf{2 0 0 , 0 0 0}$

There is much less effect due to the synthetic jet at $\mathrm{F}^{+}=1.39$. This can be attributed to the drop in $\mathrm{C}_{\mu}$. An addition test was done to see how the lift coefficient varies with $\mathrm{C}_{\mu}$. The results are shown in Figure 6.26 

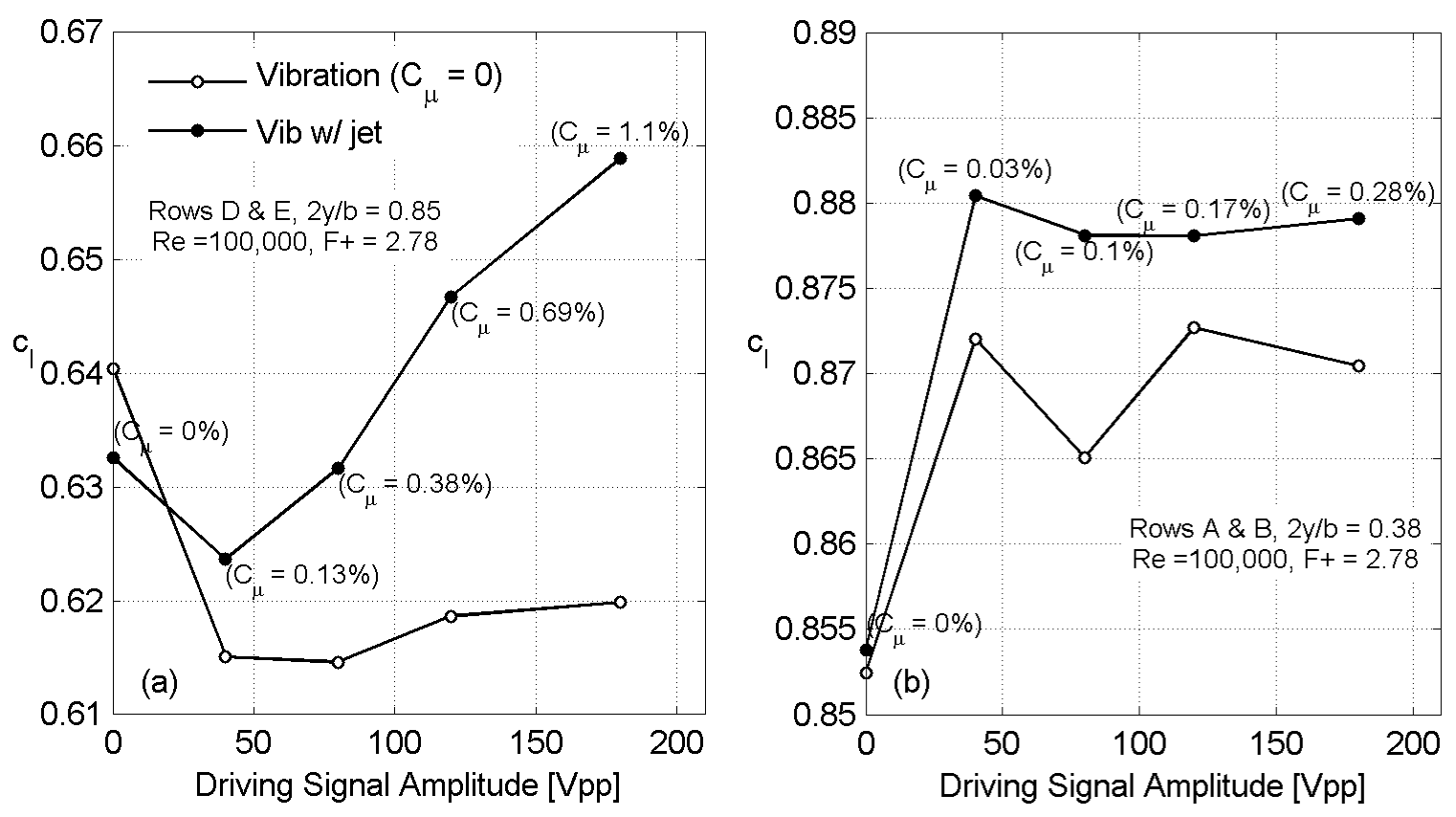

Figure 6.26 Sectional lift coefficient variation with driving signal amplitude

Figure 6.26 shows several interesting trends. In Figure 6.26 (a) the "vibration" case shows an initial drop in $\mathrm{c}_{1}$ from 0-40 Vpp, then almost no effect from 40-180 Vpp. This deserves some explanation. An increase in voltage increases the center displacement of the piezoelectric disks for each actuation cycle and causes more air to be pushed in and out of the slit. A result of this increased center displacement is an increase in vibration. A direct relationship between voltage and vibration was observed during the test by phycially feeling the wing vibrate. This suggests that only a slight amount of vibration (the amount caused by $40 \mathrm{Vpp}$ ) is needed to get the majority of the vibrational effects. After $40 \mathrm{Vpp}$ there is little effect from further increases in volatage, and thereby increases in vibration. The "vib w/ jet" case in Figure 6.26 (a) shows the combined effects of the jet and of vibration. The effect of vibration can be seen in the initial drop in $\mathrm{c}_{1}$ from 0-40 Vpp. After this initial drop it can be assumed that vibration does nothing to further decrease $c_{1}$. The increase in $c_{1}$ after $40 \mathrm{Vpp}$ is therefore caused by the synthetic jet. There is also continued increase in $\mathrm{c}_{1}$ with increasing $\mathrm{C}_{\mu}$ as the voltage increases. 
Figure 6.26 (b) shows a similar trend where vibration only has impact from 0-40 Vpp. Two interesting things can be observed from this plot. First, is that there is no increase in $c_{1}$ with increasing $\mathrm{C}_{\mu}$. This could be because the $2 \mathrm{y} / \mathrm{b}$ location where the measurements were taken is farther from the jet exit which is at $2 \mathrm{y} / \mathrm{b}=1$. Another interesting observation is that vibration caused an increase in $c_{1}$ from $0-40 \mathrm{Vpp}$ instead of a decrease as was seen in Figure 6.26 (a). It is unkown why vibration causes an increase in one location and a decrease in another. One possible explanation is that the laminar separation bubble is larger near the $2 \mathrm{y} / \mathrm{b}=0.38$ than it is at $2 \mathrm{y} / \mathrm{b}$ $=0.85$, as seen in Figure 6.16 and Figure 6.19, and vibration tends to eliminate this separation bubble. Therefore, since bubble attachment should increase lift, more of an increase in lift should be seen near $2 \mathrm{y} / \mathrm{b}=0.38$ where the laminar bubble is more prevelant. Although this would explain the increase in lift at $2 \mathrm{y} / \mathrm{b}=0.38$ from $0-40 \mathrm{Vpp}$, the decrease in lift for $2 \mathrm{y} / \mathrm{b}$ would require further investigation.

\subsection{Load Cell Results}

Because the pressure results showed vibrational effects, the load cell data was also acquired in such a way as to isolate the effect of vibration and the effect of the synthetic jet. The unactuated and actuated cases are shown in Figure 6.27. 

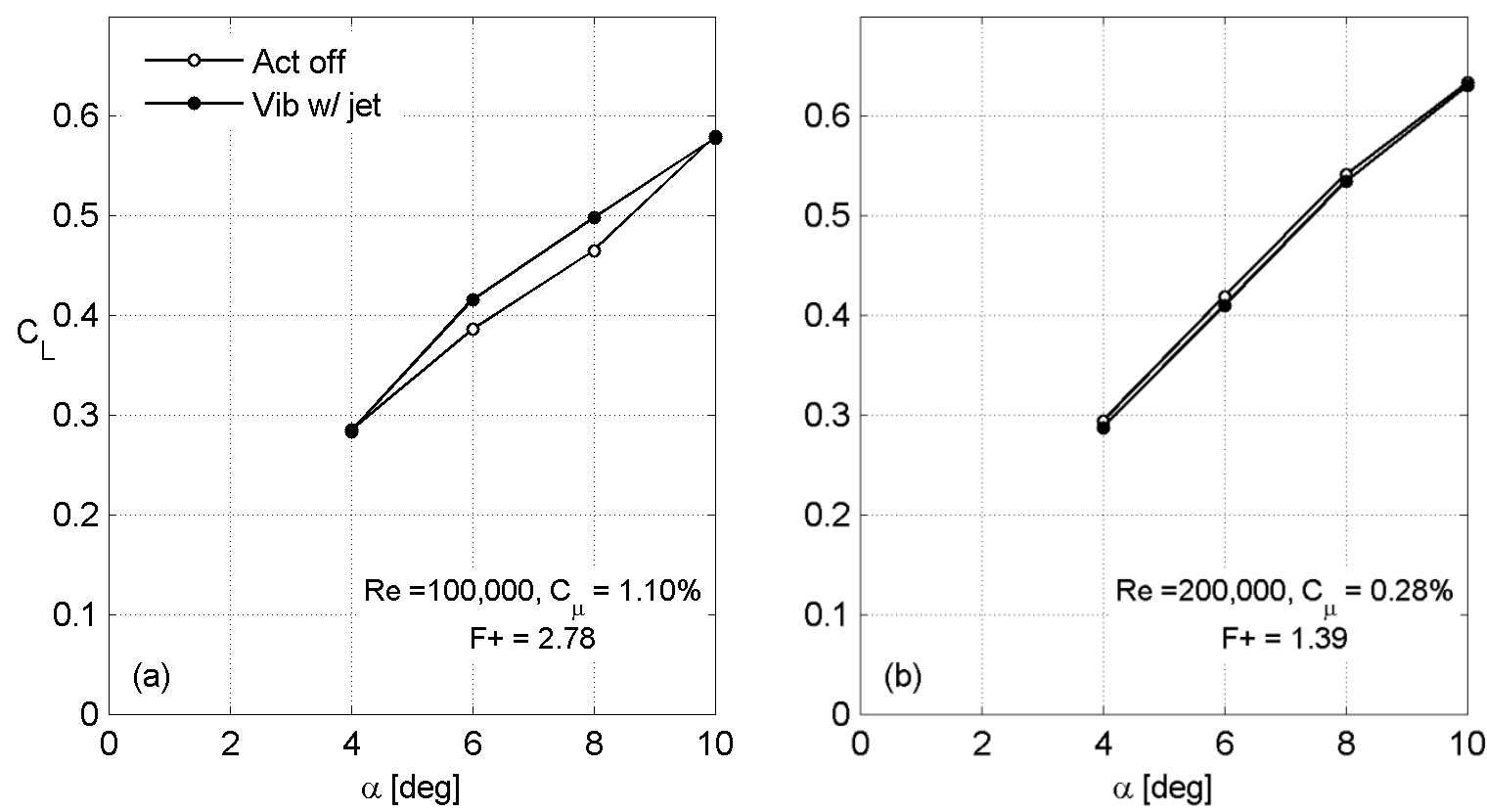

Figure 6.27 NACA 0015 lift curve for actuated and unactuated cases

From this plot, it can be seen that actuation slightly increases the lift in Figure 6.27 (a) and has almost no effect in Figure 6.27 (b). It is unclear if vibration or the jet is causing these results.

Figure 6.28 shows the isolated effect of vibration
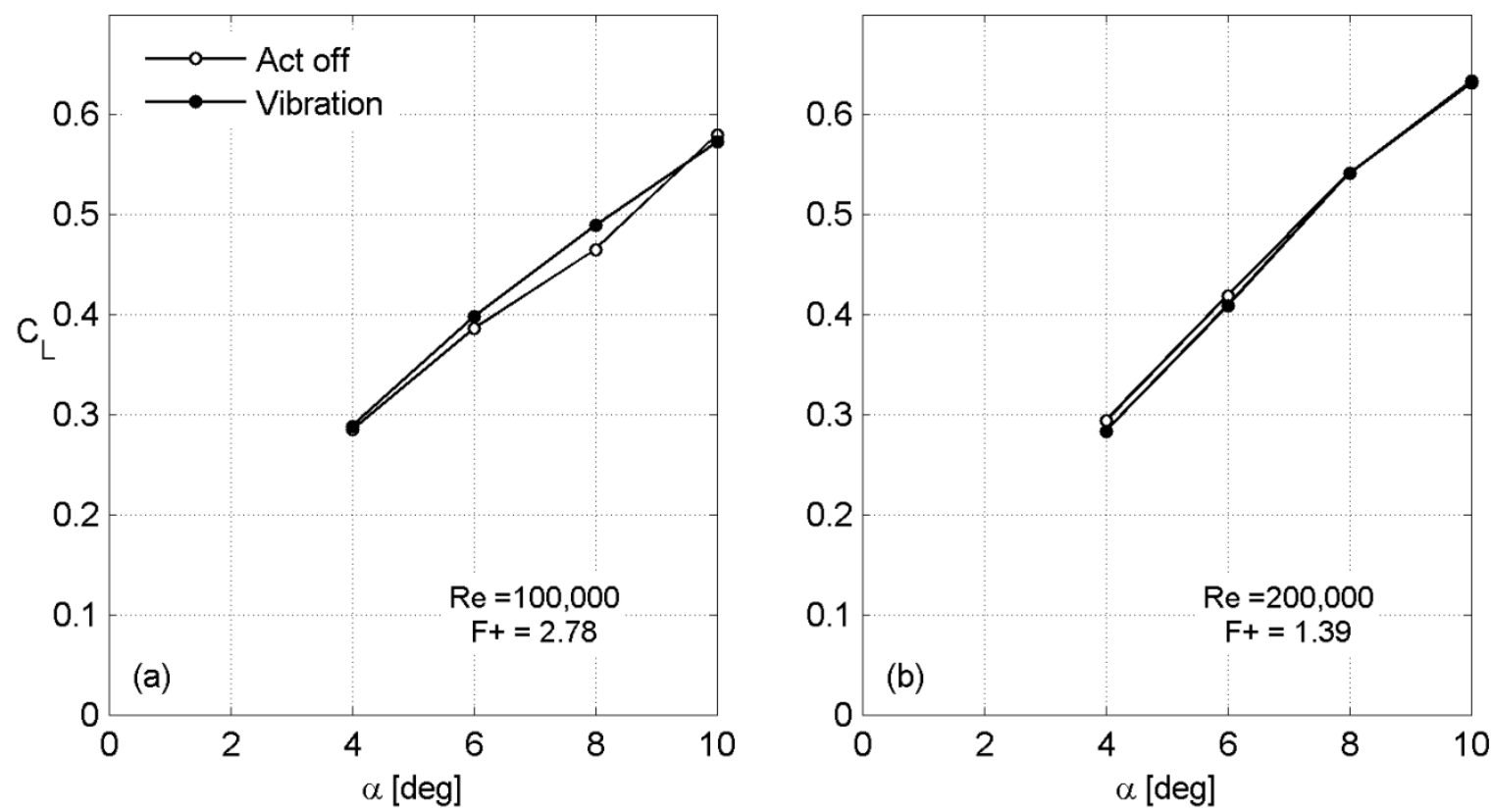

Figure 6.28 NACA 0015 lift curve showing the isolated effect of vibration 
Vibration increases the lift slightly at a Reynolds number of 100,000 although the effect is very small.. Figure 6.29 shows the effect of the synthetic jet.
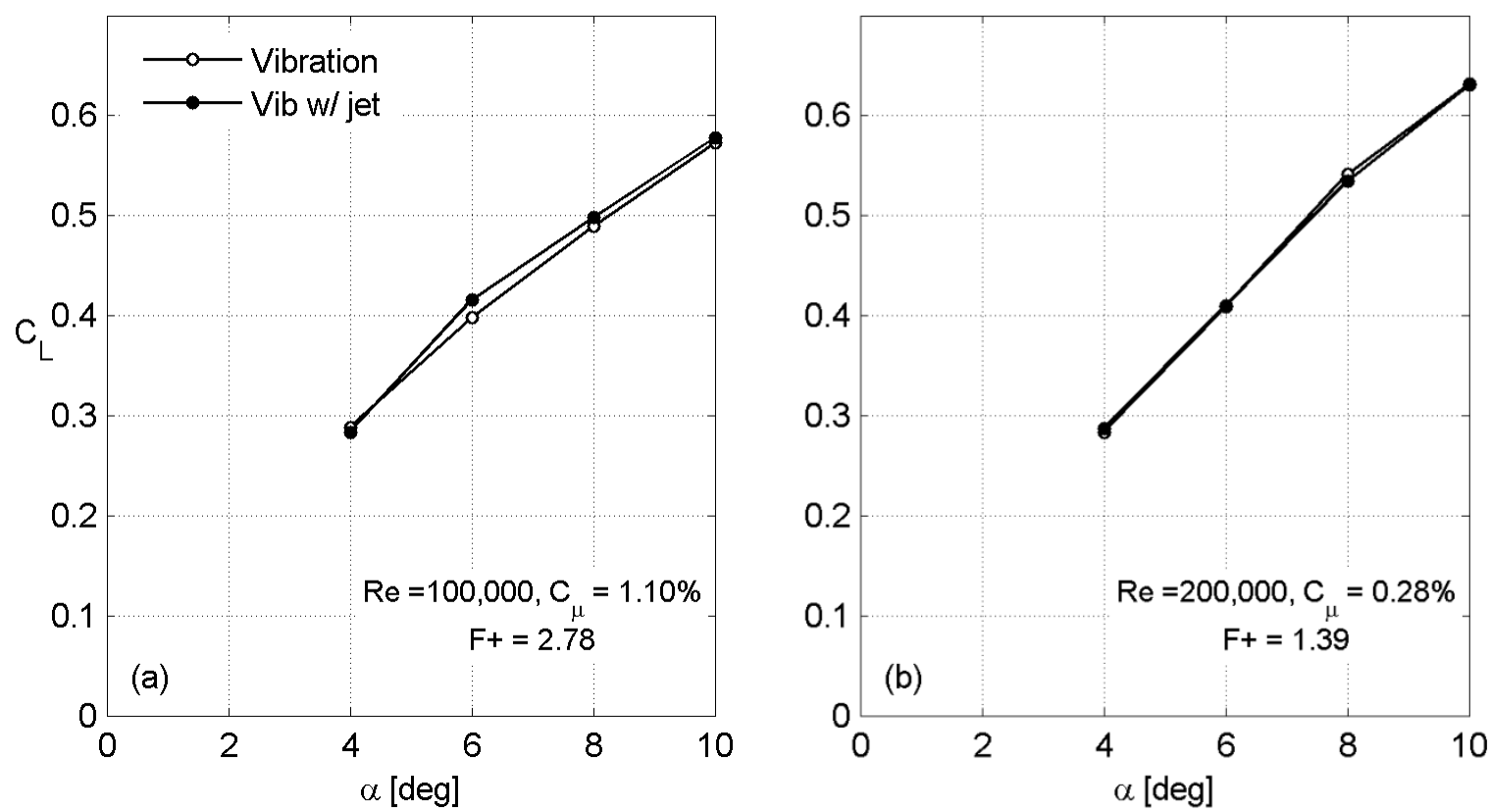

Figure 6.29 NACA 0015 lift curve showing the isolated effect of the synthetic jet

A slight increase in lift can be seen due to the synthetic jet at Figure 6.29 (a) while Figure 6.29 (b) shows no effect due to the jet. From these plots it can be concluded that actuation slightly increases the total lift on the model at the lower Reynolds number but has little to no effect at higher Reynolds numbers. Although, this is most likely due to the lower $C_{\mu}$ at $\operatorname{Re}=$ 200,000. The drag for the unactuated and actuated cases is shown in Figure 6.30. 

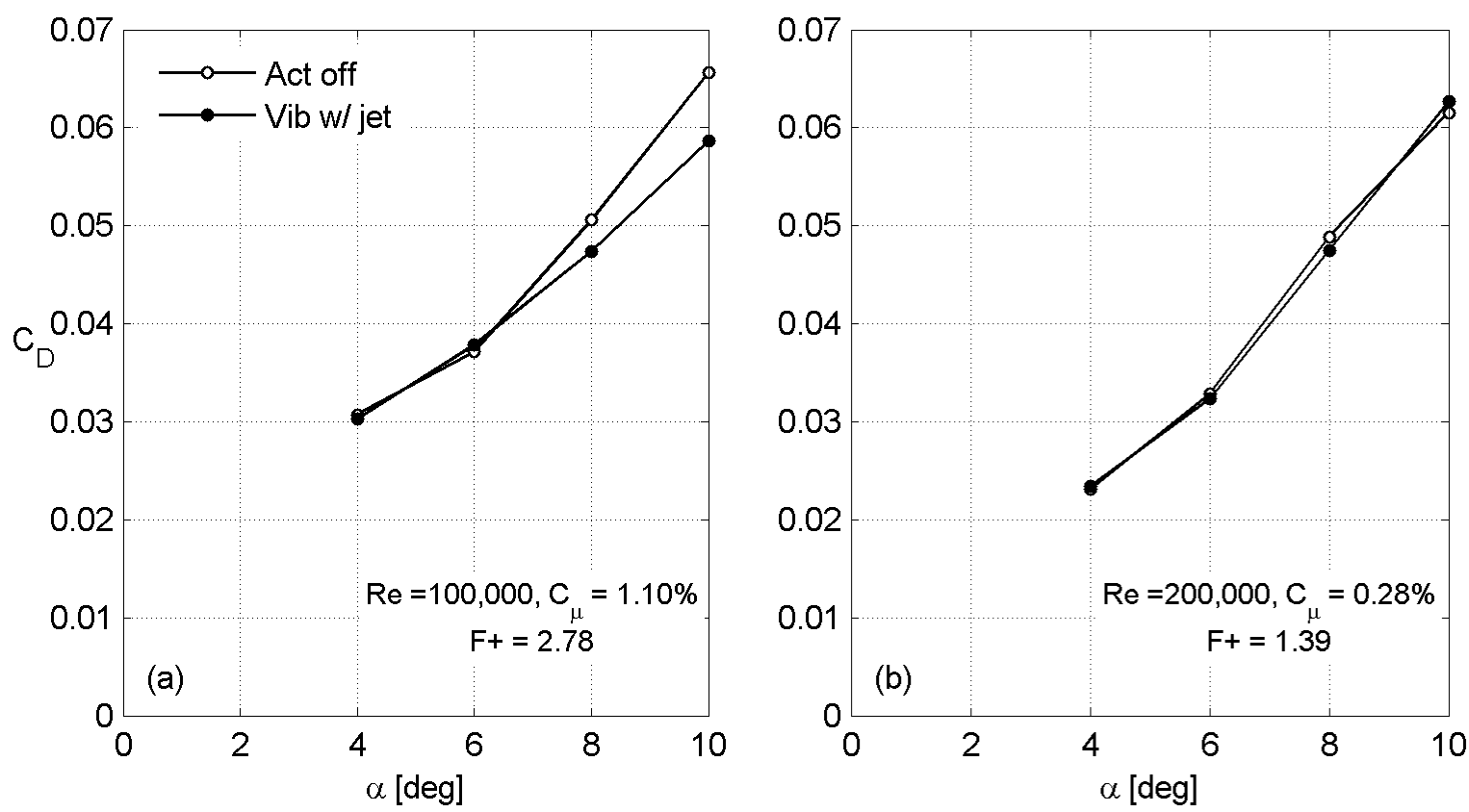

Figure 6.30 NACA 0015 drag curve for actuated and unactuated cases

As is seen in this figure, it is unclear what effect is due to vibration and what effect is due to the synthetic jet. Figure 6.31 shows the isolated effect of vibration
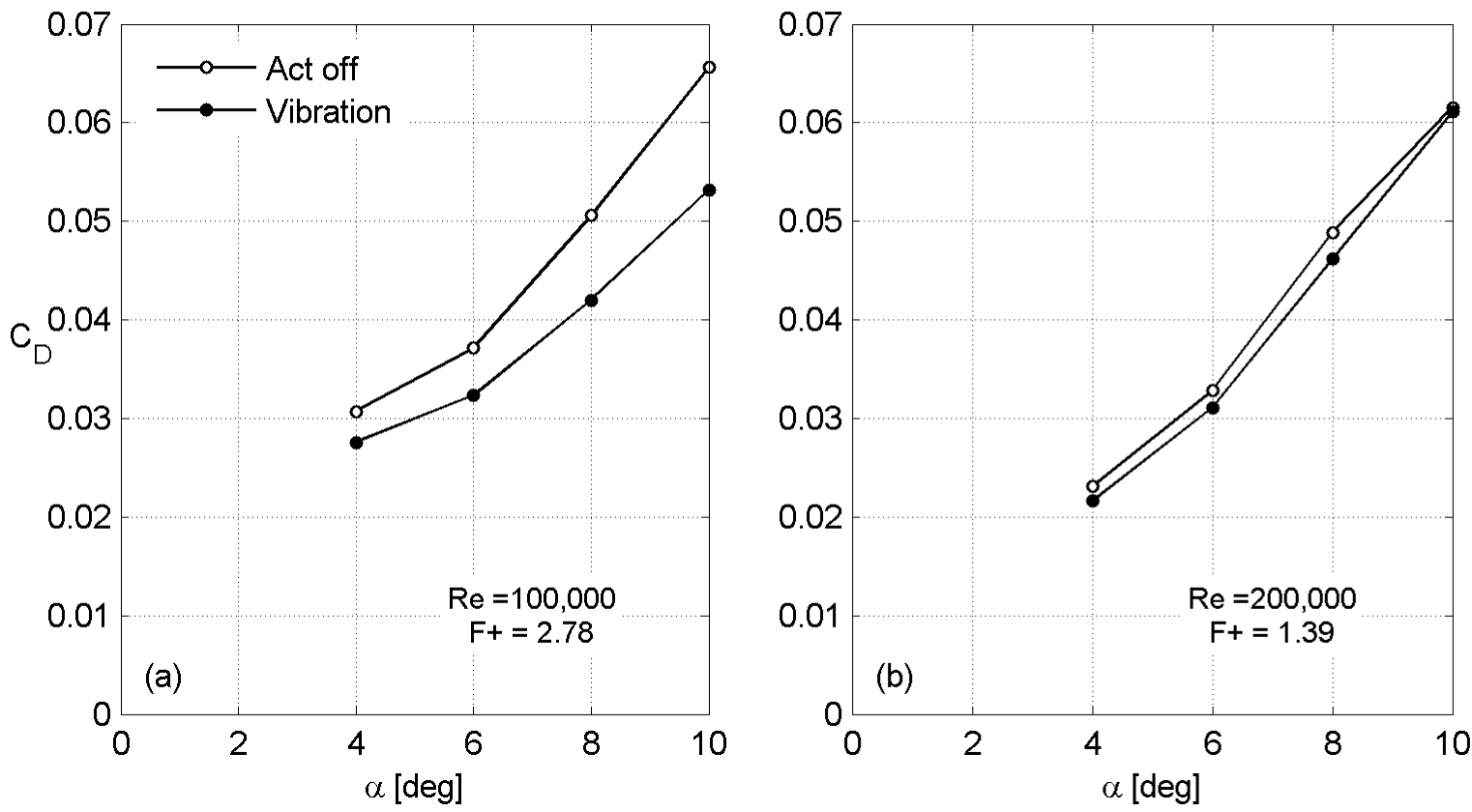

Figure 6.31 NACA 0015 drag curve showing the isolated effect of vibration 
There is a significant decrease in drag in Figure 6.31 (a). This results was unexpected, the reason for this decrease in drag is unknown. There is also a slight decrease in drag in Figure 6.31 (a). Figure 6.32 shows the isolated effect of the synthetic jet.
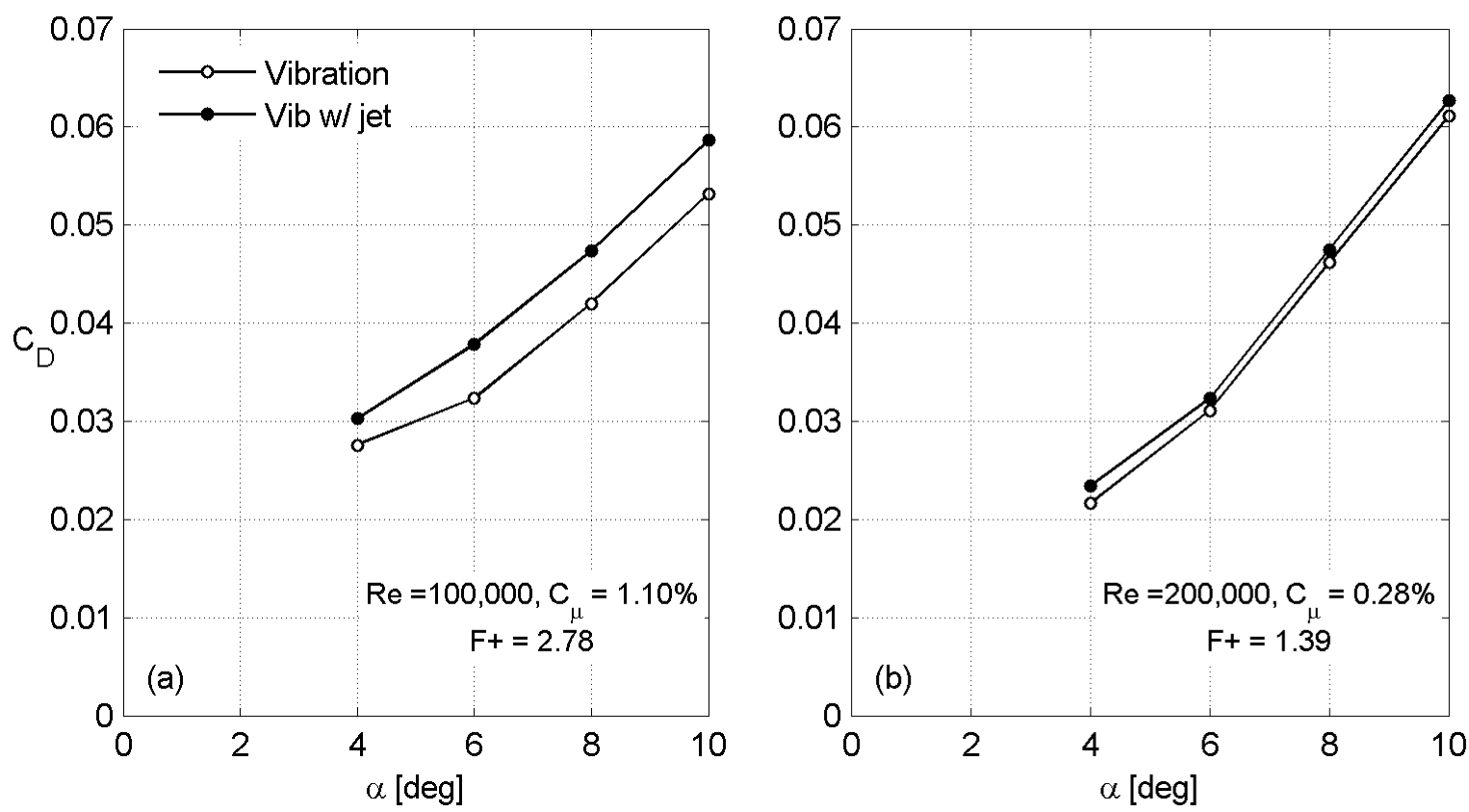

Figure 6.32 NACA 0015 drag curve showing the isolated effect of the synthetic jet

These results were opposite of what was predicted for this Thesis. The expected result was that the synthetic jet would decrease the drag in a similar way to other wingtip devices although these results show the opposite effect. The jet increased the drag. Figure 6.32 (a) shows a significant increase in drag while Figure 6.32 (b) shows a slight increase in drag which can be attributed to the lowered $\mathrm{C} \mu$.

The lift and drag were used to see how actuation and vibration affect L/D and the induced drag. Figure 6.33-Figure 6.35 show the L/D for each actuator configuration 

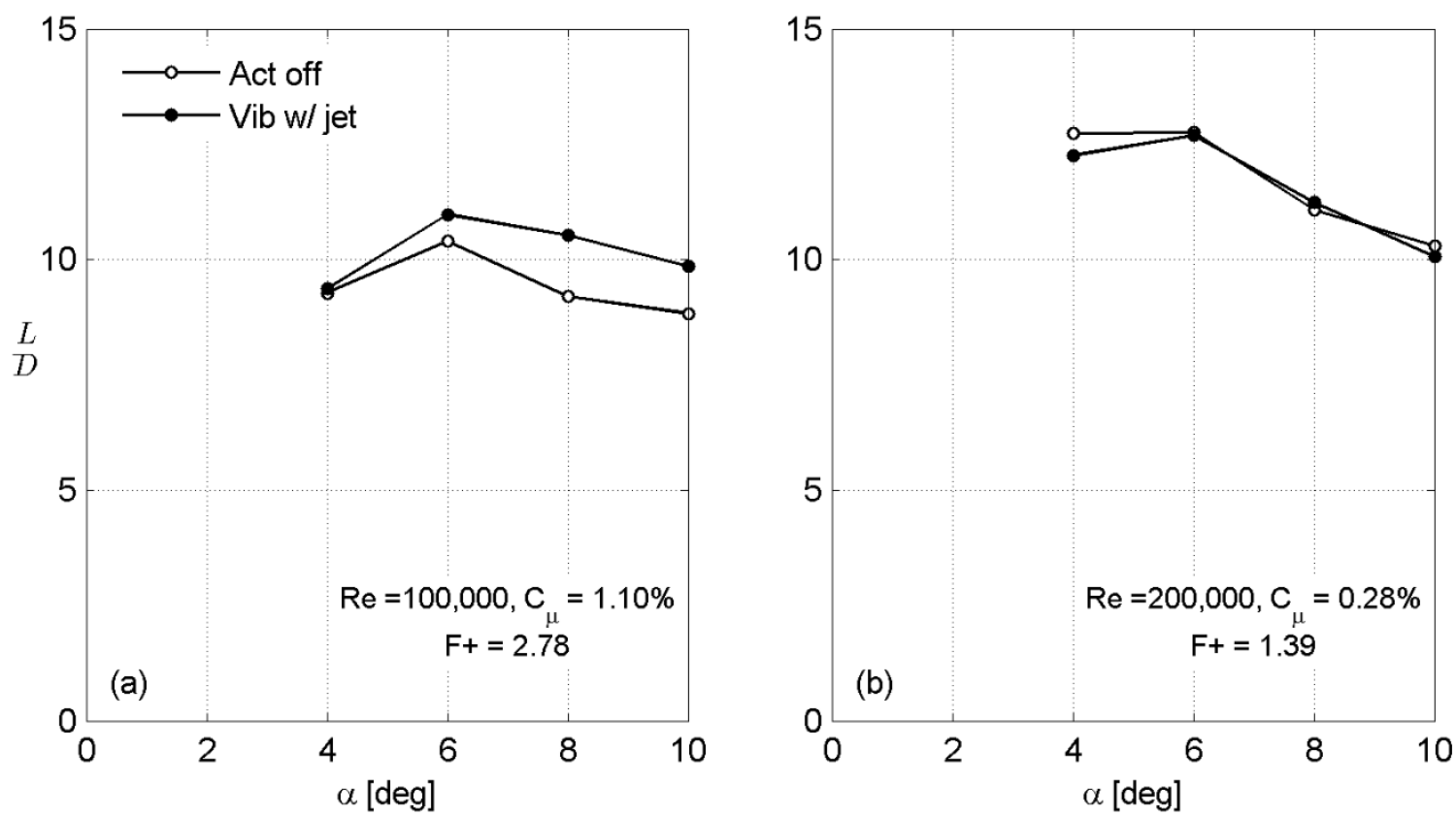

Figure 6.33 NACA $0015 \mathrm{~L} / \mathrm{D}$ curve for actuated and unactuated cases
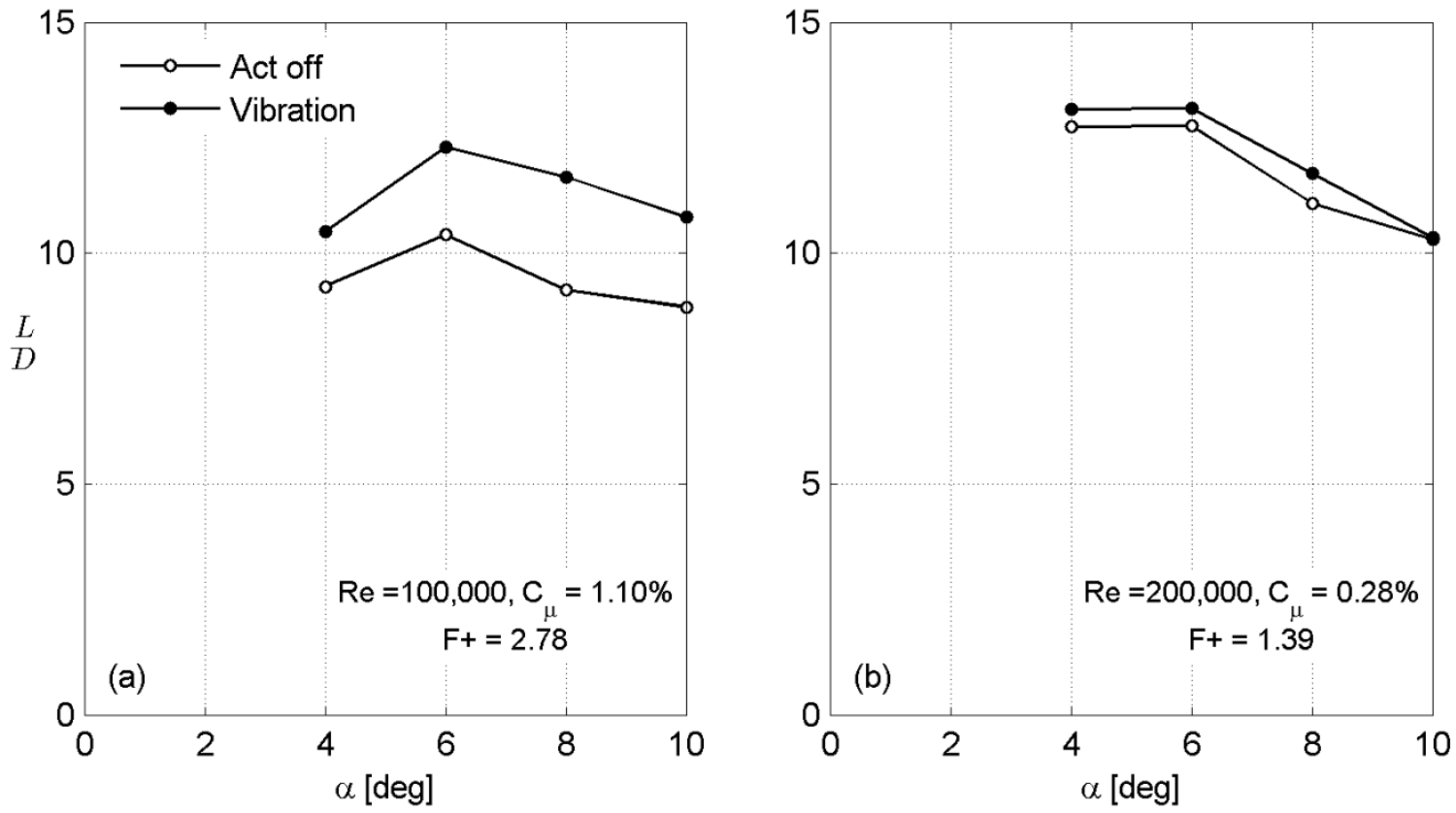

Figure 6.34 NACA $0015 \mathrm{~L} / \mathrm{D}$ showing the isolated effect of vibration 

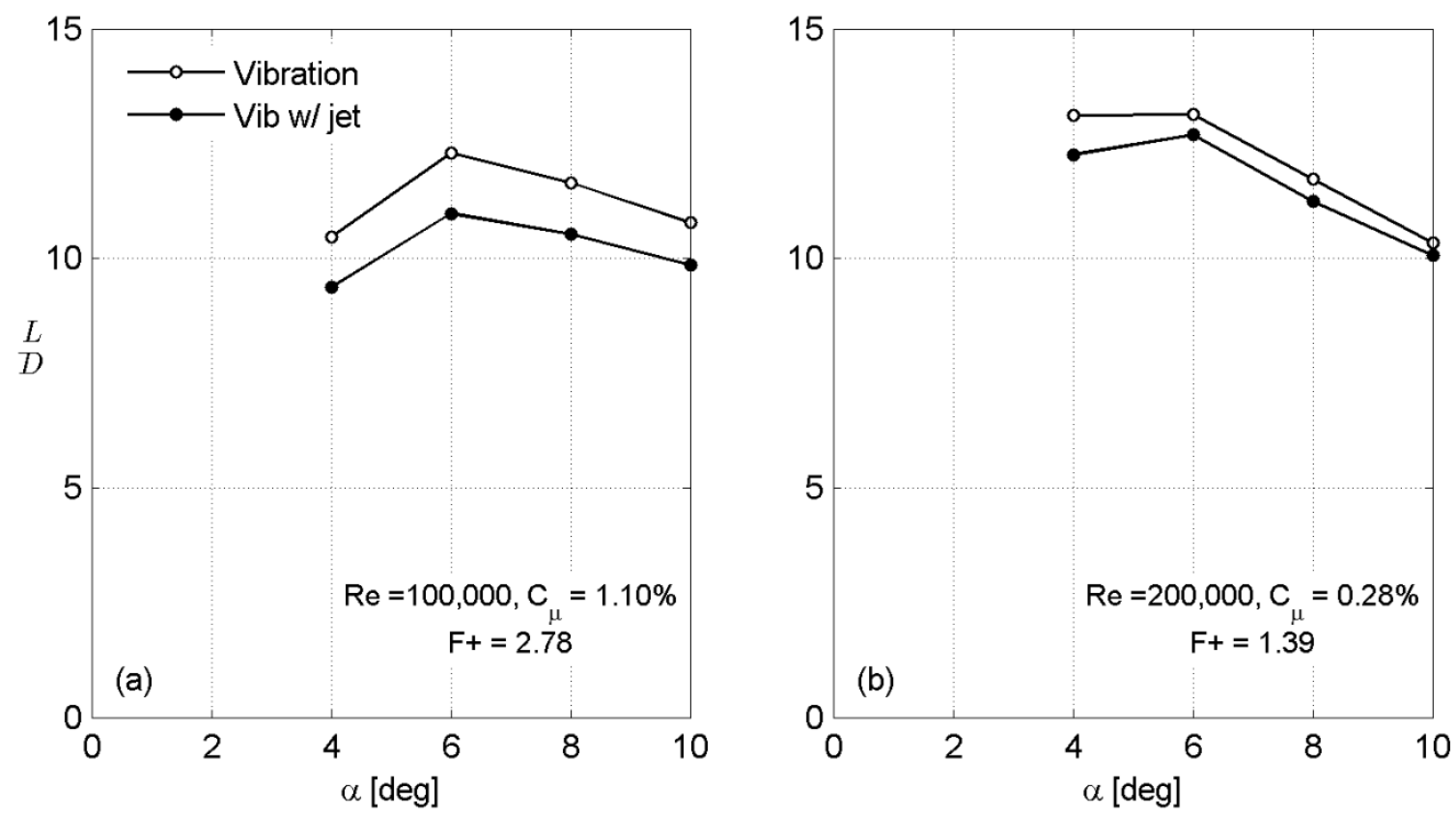

Figure 6.35 NACA 0015 L/D curve showing the isolated effect of the synthetic jet

Figure 6.33 shows that the cumulative effect of vibration and the jet does not produce any significant results. Figure 6.34-Figure 6.35 show that vibration increases L/D while the synthetic jet decreases L/D. The induced drag curves are shown in Figure 6.36 through Figure 6.38. 

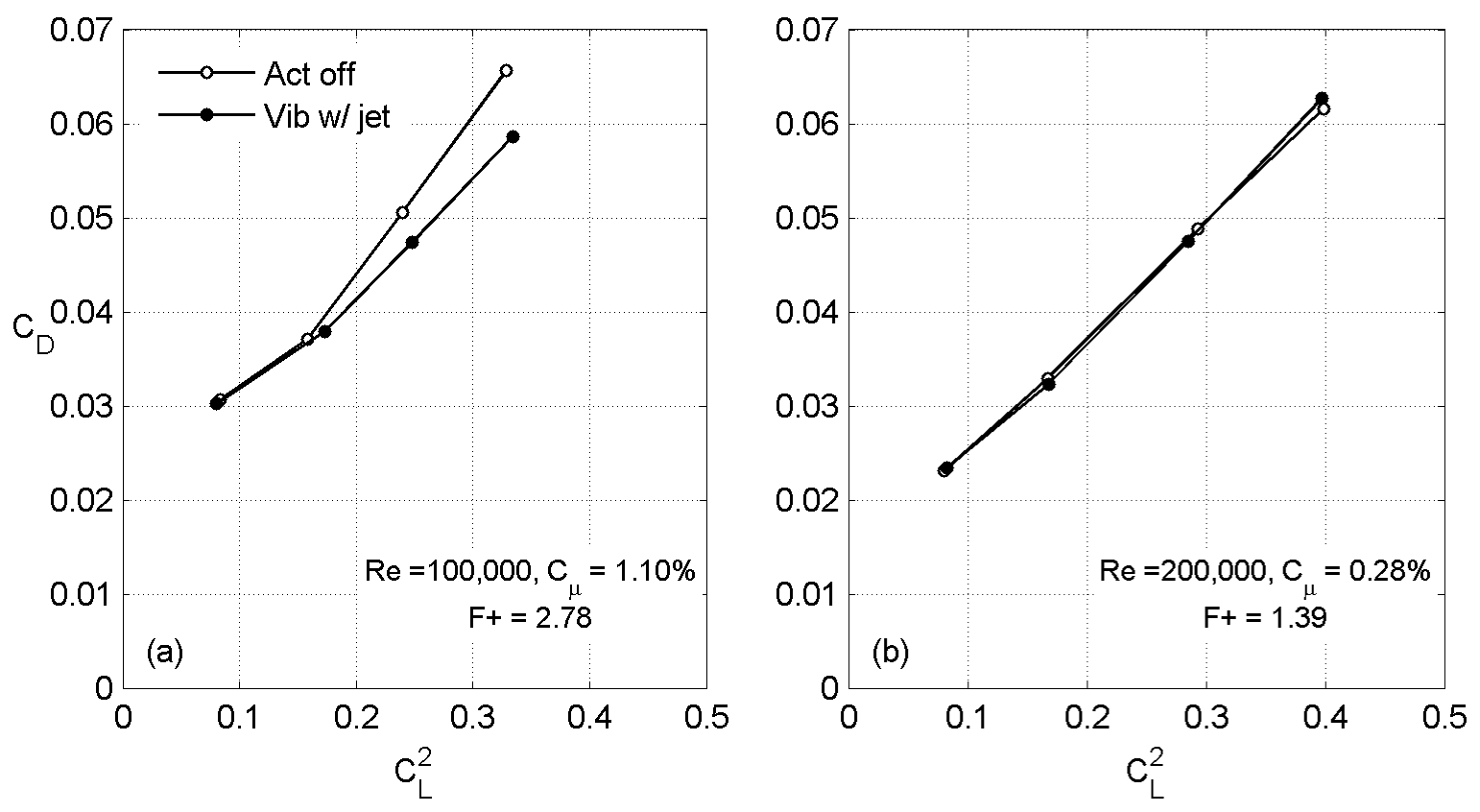

Figure 6.36 NACA $0015 C_{D}$ vs. $C_{L}{ }^{2}$ curve for actuated and unactuated cases
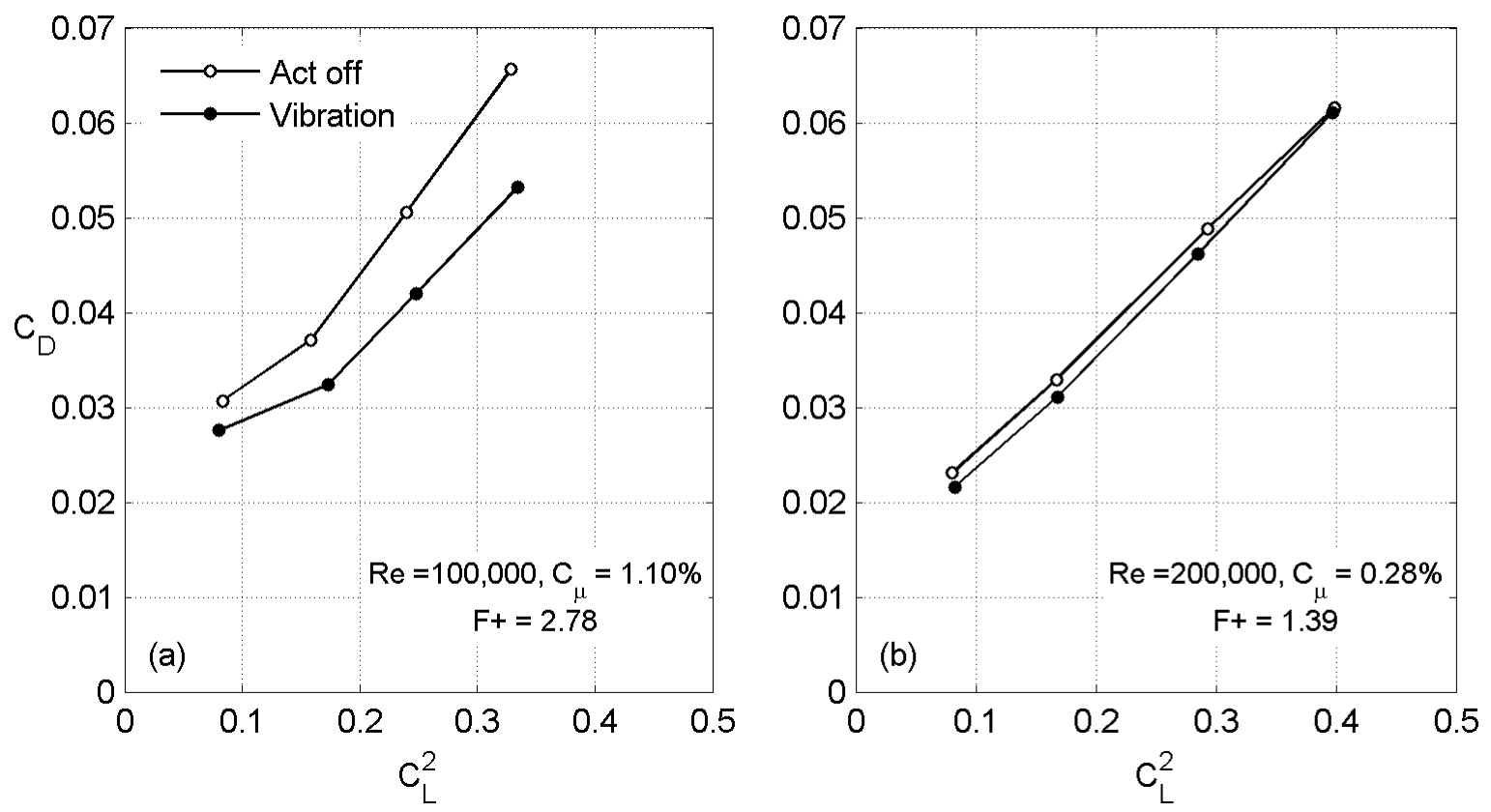

Figure 6.37 NACA $0015 C_{D}$ vs. $C_{L}^{2}$ curve showing the isolated effect of vibration 

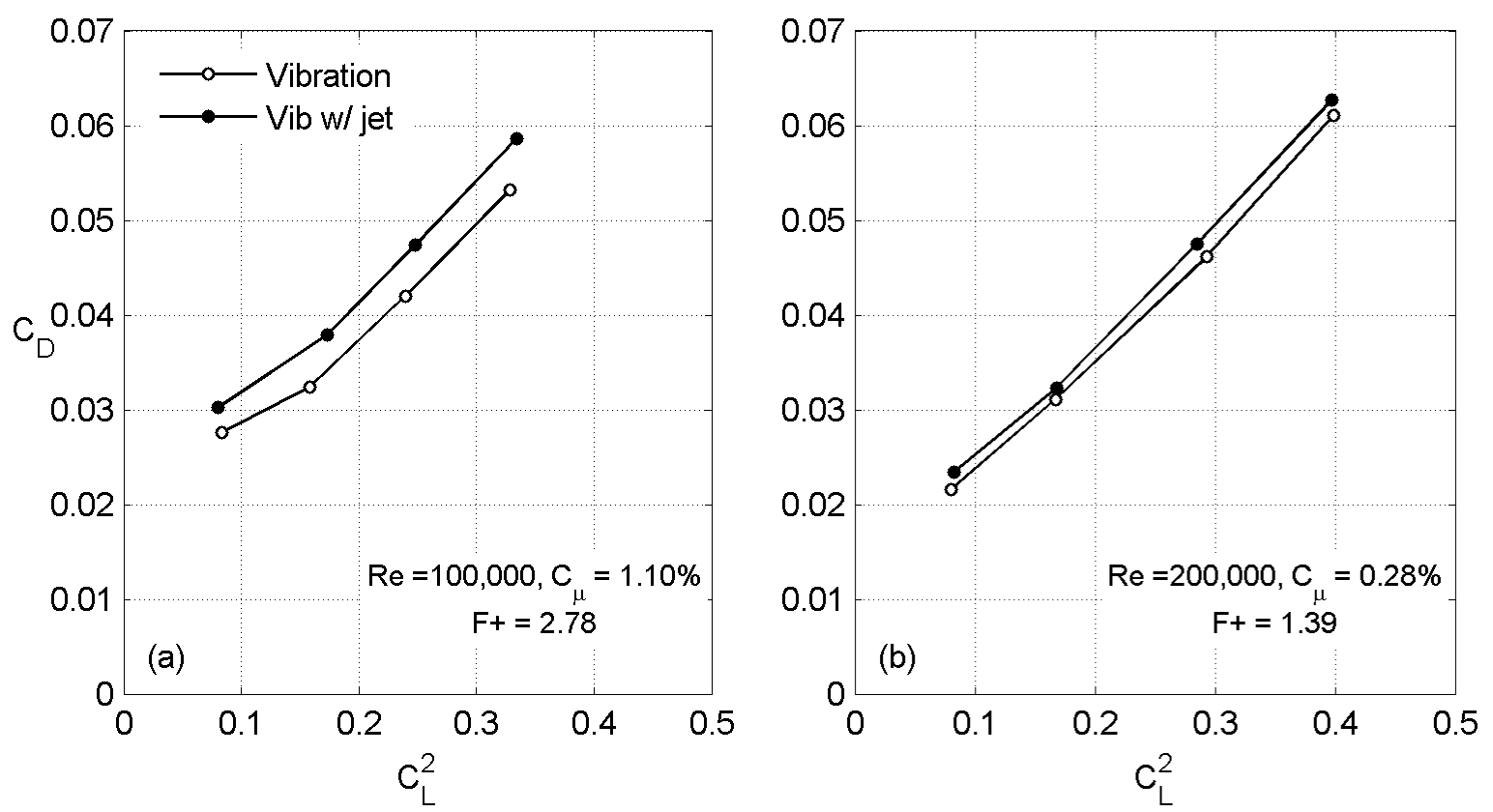

Figure 6.38 NACA $0015 C_{D}$ vs. $C_{L}{ }^{2}$ curve showing the isolated effect of the synthetic jet

Little can be concluded from Figure 6.36 since it contains both the effect of vibration and the effect of the synthetic jet. Figure 6.37 (a) possibly shows a significant finding. If the linear portions of the curves in Figure 6.37 (a) are extended, they both interact at about $C_{D}=0.01$ at $C_{L}$ $=0$. The slope of the vibration curve has a slightly decreased slope. This means that vibration should reduce the induced drag by up to as $11.3 \%$ at $\alpha=4^{\circ}$ to $23.4 \%$ at $\alpha=10^{\circ}$. This decrease in drag was also seen in Figure 6.31. Interestingly, Figure 6.16 did not show any change in the $C_{p}$ distribution near the wingtip due to vibration which does not support a reduction in induced drag. Error analysis was done in Appendix B to see if the results in Figure 6.37 were significant. As seen in Figure 7.4, the errorbars show that the error in the drag measurement at $\operatorname{Re}=10^{5}$ is too high to make any definite conclusions about the induced drag reduction using the load cell data. The synthetic jet results in Figure 6.38 show that there is no change in slope although $\mathrm{C}_{\mathrm{D} 0}$ is increased due to the synthetic jet for both Reynolds numbers. This means that the synthetic jet actually increases the total drag rather than decreases the induced drag as was predicted at the start of this Thesis. 


\subsection{Oil Film Interferometry Results}

The oil film results for the suction surface are shown in Figure 6.39.
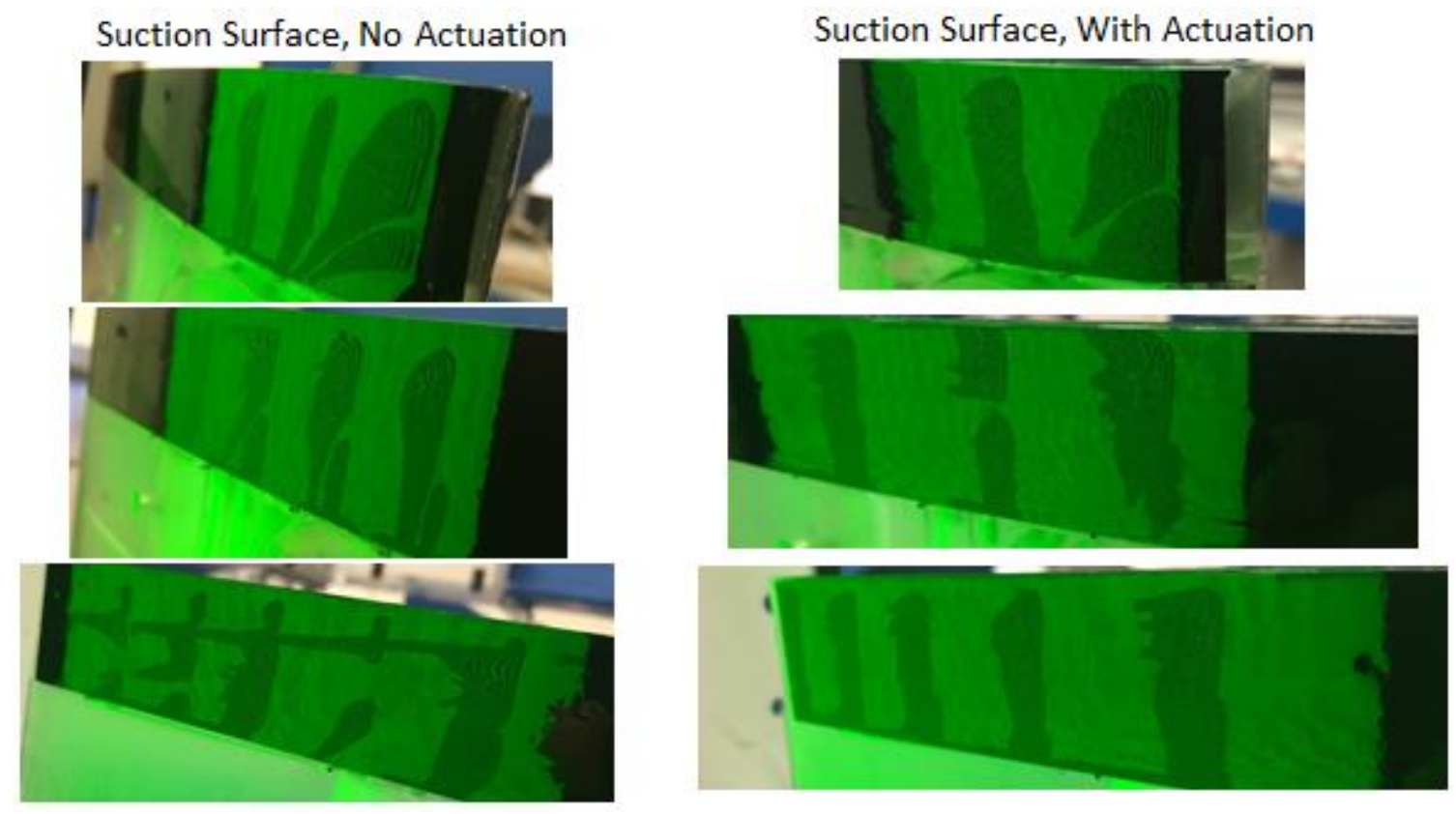

Figure 6.39 Oil film interferometry on suction surface $\left(\operatorname{Re}=100,000 \alpha=10^{\circ}\right)$.

The images on the left show the suction surface without actuation. The top picture starts with the leading edge and moves down towards the trailing edge. The pictures on the right shows the same thing except with actuation at $\mathrm{C}_{\mu}=1.10 \%$, and $\mathrm{F}^{+}=2.78$. The footprint of the tip vortex can be seen in the unactuated case. This vortex starts at about $\mathrm{x} / \mathrm{c}=0.3$ and moves downstream and inboard further down the wing. This footprint is caused by the low pressure region created by the vortex. When the actuators were turned on, the footprint of the vortex was eliminated. This suggests a that the vortex was pushed off the wingtip or the vortex became so diffuse that it could not produce low enough pressure to cause the droplets to come together and form the footprint. Both of these conjectures would reduce the downwash and hence the induced drag should be reduced. This is consistent with Figure 6.36 which showed a decrease in induced drag when actuation was triggered. This is a significant result as both flow visualization and direct drag measurements support a decrease in induced drag. Figure 6.40 shows the pattern on the tip surface. 


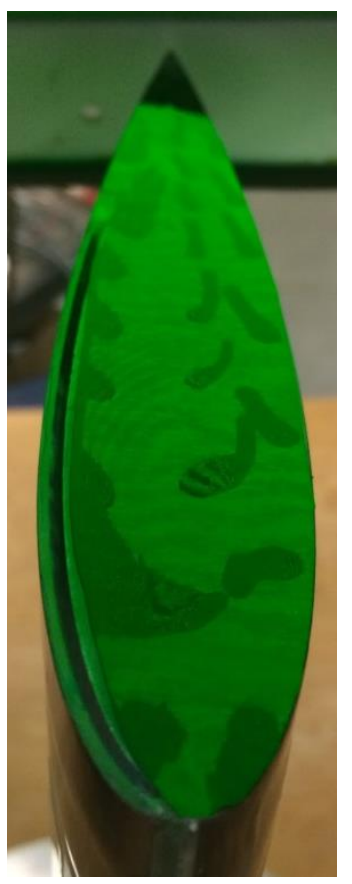

(a)

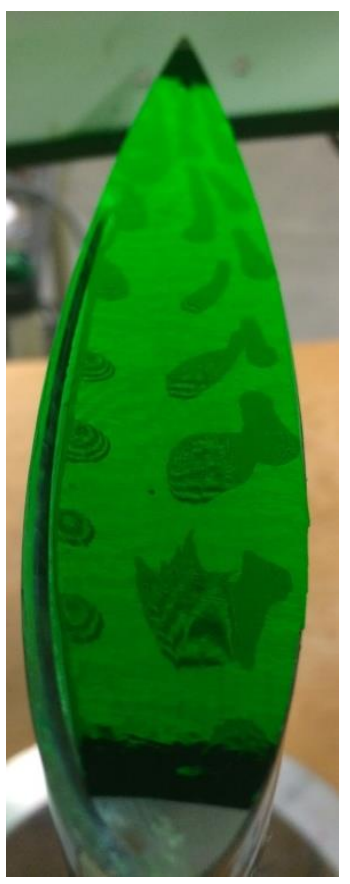

(b)

Figure 6.40 Oil film interferometry on tip surface $\left(\operatorname{Re}=100,000 \alpha=10^{\circ}\right)$. (a) Actuators off. (b) Actuators on slit uncovered

Actuation seemed to have little effect on the tip surface. There is a secondary vortex forming from the pressure surface to the tip surface. This vortex was not affected by actuation, only the primary vortex on the suction surface was affected.

\subsection{Stethoscope Boundary Layer Investigation Results}

The pressure surface of the model was laminar for both $\operatorname{Re}=100,000$ and $\operatorname{Re}=200,000$ independent of whether the model was acutated. The suction surfaces transitioned from laminar to turbulent in different locations depending on the Reynolds number. Actuation seemed to have very little effect on the transition point. The transition locations are shown in Table 6.2.

Table 6.2 Laminar to turbulent transition locations $\alpha=\mathbf{1 0}^{\circ}$

\begin{tabular}{|c|c|c|c|c|c|c|}
\hline & \multicolumn{3}{|c|}{$\operatorname{Re}=100,000$} & \multicolumn{3}{c|}{$\operatorname{Re}=200,000$} \\
\cline { 2 - 7 } & Act off & Vibration & Vib w/ jet & Act off & Vibration & Vib w/ jet \\
\hline $\begin{array}{c}\text { Rows A \& } \\
\text { B }\end{array}$ & $25 \%$ & $25 \%$ & $25 \%$ & $12 \%$ & $16 \%$ & $16 \%$ \\
\hline
\end{tabular}




\begin{tabular}{|c|c|c|c|c|c|c|}
\hline $\begin{array}{c}\text { Rows D \& } \\
\text { E }\end{array}$ & $30 \%$ & $25 \%$ & $25 \%$ & $14 \%$ & $16 \%$ & $16 \%$ \\
\hline
\end{tabular}

It can be seen that the transition point moved upstream as the Reynolds number was increased but stayed relatively consistent with actuator configuration. These results show that the effects of the different actuator configurations are not due to a change in transition location. 


\section{Conclusion}

An experiment was designed and manufactured to test active flow control on a NACA 0015 semispan model. Piezoelectric actuators were used in series to create a synthetic jet that would eminate from the wingtip of the NACA 0015 model. A load cell/slider system, pressure ports, and oil droplet flow visualization were all used to study the effects of the synthetic jet on the NACA 0015 model at various Reynolds numbers and angles of attack.

Although the synthetic jet, without the added effect of wing vibration, had little effect on net lift and slightly increased the net drag, there was still a significant change in the flowfield near the wingtip. The synthetic jet with vibration decreased the drag only slightly while vibration alone decreased drag significantly from $11.3 \%$ at $\alpha=4^{\circ}$ to $23.4 \%$ at $\alpha=10^{\circ}$. Pressure ports placed near the wingtip showed increased suction when the jet was turned on. Oil flow visualization was also used to see if the jet had any visible affect on the flow pattern near the tip. The results showed that the vortex footprint would disappear when the jet was turned on . This suggests that the synthetic jet either diffused the vortex to such an extent that a footprint did not appear, or that the jet pushed the vortex outboard and off the wingtip. Pressure port data showed that vibration eliminates the laminar separation bubble that occurs on a non-tripped wing at Reynolds numbers used in this Thesis. In summary, the major conclusions of this Thesis are:

1. Vibration of the NACA 0015 semispan model alone has shown a significant potential decrease in drag from $11.3 \%$ at $\alpha=4^{\circ}$ to $23.4 \%$ at $\alpha=10^{\circ}$. However, because of the limitation of the load cell, further testing with a more accurate drag balance is recommended. 
2. The synthetic jet has a significant effect on the flow field near the wingtip. There was a signifant increase in $-\mathrm{C}_{\mathrm{p}}$ at $2 \mathrm{y} / \mathrm{b}=0.85$ and surface oil flow visualization showed absence of the wingtip vortex footprint with actuation.

3. Pressure port data showed that the laminar separation bubble is eliminated due to wing vibration

4. The synthetic jet without vibration is not effective at reducing induced drag in the configuration used in this thesis, showing an increase in drag rather than a decrease. 


\section{Bibliography}

1 Thomas, A. S., “Aircraft Drag Reduction Technology-A Summary”. Advisory Group for Aerospace Research and Development (AGARD), Report 723, Belgium, 1985.

${ }^{2}$ Zimmer, H., "Aerodynamic Optimization of Wings at Subsonic Speeds and the Influence of Wingtip Design”, NASA TM-88534, 1983

${ }^{3}$ Kroo, I. "Nonplanar Wing Concepts for Increased Aircraft Efficiency". VKI Lecture Series on Innovative Configurations and Advanced Concepts for Future Civil Aircraft, Stanford, 2005 .

${ }^{4}$ Rossow, V. J., and James, K. D., “Overview of Wake-Vortex Hazards During Cruise,” Journal of Aircraft, Vol. 37, No. 6, 2000, pp. 960-975. doi:10.2514/2.2723

5 Filippone, A., "Analysis of Carbon-Dioxide Emissions from Transport Aircraft," Journal Aircraft, Vol. 45, No. 1, Jan.-Feb. 2008, pp. 185-197. doi:10.2514/1.31422

${ }^{6}$ Whitcomb, R. T., “A Design Approach and Selected Wind-Tunnel Results at High Subsonic Speeds for Wing-Tip Mounted Winglets”, NASA-TN-D-8260, 1976

${ }^{7}$ KROO, I. "Drag due to lift: Concepts for prediction and reduction", Annual Rev: Fluid Mechanics, 2001, 33, pp 587-617.

${ }^{8}$ McLean D., "Wingtip Devices: What they Do and How They Do it," presented at the Boeing Performance and Flight Operations Engineering Conference 2005.

${ }^{9}$ Mineck, R.E., "Study of potential aerodynamic benefits from spanwise blowing at wingtip", NASA technical report 3515, 1995.

${ }^{10}$ Seifert A., Darabi A., and Wygnanski, I., "Delay of Airfoil Stall by Periodic Excitation", Journal of Aircraft, Vol. 33, No. 4, 1996, 691-698. 
${ }^{11}$ Smith, B.L. and Swift, G.W., "A comparison between synthetic jets and continuous jets,", Experiments in Fluids, Vol. 34, pp. 467-472, 2003.

${ }^{12}$ Margaris, P., and Gursul, I., "Wing Tip Vortex Control Using Synthetic Jets," The Aeronautical Journal, Vol. 110, No. 1112, 2006, pp. 673-681.

${ }^{13}$ Boesch, G., Vo, H., Savard, B., Wanko-Tchatchouang, C., and Mureithi, N., "Flight control using wing-tip plasma actuation," Journal of Aircraft, Vol. 47, No. 6, 2010, pp. 1836-1846.

${ }^{14}$ Chappell S., Angland D., "Active Control of a Wing Tip Vortex with a Dielectric Barrier Discharge Plasma Actuator" AIAA Paper 2012-2952, 6th AIAA Flow Control Conference, New Orleans, Louisiana, 25-28 June 2012, 10.2514/6.2012-2952

15 S.I. Green, "Fluid Vortices" in: Fluid Mechanics and Its Applications, Kluwer Academic Publisher, 1995, pp. 427-470.

${ }^{16}$ Giuni, M., and Green, R.B. "Vortex formation on squared and rounded tip," Aerospace Science and Technology, 29 (1). pp. 191-199

${ }^{17}$ S. Gao, J.Z. Zhang, X.M. Tan "Experimental study on heat transfer characteristics of synthetic jet driven by piston actuator" Sci. Chin. Tech. Sci., 55 (2012), pp. 1732-1738

${ }^{18}$ Crittenden, T. and Glezer, A., “A High-Speed Compressible Synthetic Jet," Physics of Fluids, Vol. 18, No. 1, 017107, 2006

${ }^{19}$ J. S. Agashe, M. Sheplak, D. P. Arnold, and L. N. Cattafesta, "MEMS-based Actuators for Flow-Control Applications," in IUTAM Symposium on Flow Control and MEMS, J. F. Morrison, Ed.: Springer, September 2006, pp. 25-32.

${ }^{20}$ Lopez, O. and Moser, R., "Computational Study of a NACA4415 airfoil using synthetic jet control", Ph.D. thesis, The University of Texas at Austin, 2009 
${ }^{21}$ Mane, P., Mossi, K., and Bryant R., "Synthetic Jets with Piezoelectric Diaphragms". Proceedings of SPIE Vol. 5761.

${ }^{22}$ P. Mane, "Experimental Design and Analysis of Piezoelectric Synthetic Jets in Quiescent Air", Masters Thesis, Virginia Commonwealth University, Fall 2005.

${ }^{23}$ Piezo Systems, INC. http://www.piezo.com/prodbm5disk.html (accessed July 2, 2013)

${ }^{24}$ Omega Engineering. http://www.omega.com/Pressure/pdf/LCFD.pdf (accessed December 2013)

${ }^{25}$ http://www.tsi.com/uploadedFiles/_Site_Root/Products/Literature/Catalogs/Hotwire_Catalog_2 980465.pdf

${ }^{26}$ Barlow, J. B. Rae, W. H. Pope, A., Low Speed Wind Tunnel Testing, Third Edition, John Wiley and Sons, Inc., (1999)

${ }^{27}$ Jacobs, Eastman N Sherman, Albert. “Airfoil section characteristics as affected by variations of the Reynolds number”. NACA Report 586, (1937).

${ }^{28}$ Leon L., "Experimental Testing of Low Reynolds Number Airfoils for Unmanned Aerial Vehicles”, Masters Thesis, Institute for Aerospace Studies University of Toronto, 2013

${ }^{29}$ D. Greenblatt, and A. E. Washburn, "Influence of Finite Span and Sweep on Active Flow Control Efficacy”. AIAA Journal 46:7, 1675-1694, 2003

${ }^{30}$ J.F. Marchman, C.L Gunther and J.F Gundlach, "Semi Span Testing at Low Reynolds number", AIAA 36th Aerospace Science Meeting and Exhibits, January 12-15, 1998, Reno, NV.

${ }^{31}$ Lee, T., Birch, D., Mokhtarian, F., and Kafayeke, F., "Structure and Induced Drag of a Tip Vortex,” Journal of Aircraft, Vol. 41, No. 5, 2004, pp. 1138-1145. doi:10.2514/1.2707 


\section{Appendices}

\section{A Sample Calculations}

Reynolds number (temperature of $64^{\circ} \mathrm{F}$ )

$R e=\frac{u c}{v}=\frac{24 * 8 / 12}{1.6 e-04}=100,000$

\section{Dynamic pressure correction}

$K_{q}=\frac{q_{A}}{q_{\text {tunnel }}}=\frac{3.8146 * 10^{-3}}{3.8090 * 10^{-3}}=1.0015$

Static pressure correction

$K_{p}=\frac{p_{A}}{p_{\text {tunnel }}}=\frac{-7.0010 * 10^{-3}}{-6.9185 * 10^{-3}}=1.0119$

Total pressure correction

$K_{t}=\frac{p_{t, A}}{p_{t, \text { tunnel }}}=\frac{-3.1864 * 10^{-3}}{-3.1095 * 10^{-3}}=1.0247$

Pressure coefficient $(\operatorname{Re}=100,000 \alpha=10$ Row $C x / c=2.1 \%)$

$C_{p}=\frac{p_{i}-K_{p} p_{\text {tunnel }}}{K_{\varepsilon} K_{q}\left(p_{t, \text { tunnel }}-p_{\text {tunnel }}\right)}=\frac{-0.0153-(1.0119)(-0.0069)}{(1.0241)(1.0015)(-0.0031-(-0.0069))}=-2.1342$

Wing planform area

$S=b c=2(16.813 * 8) \frac{1}{144}=1.88 f t^{2}$

$\frac{S}{2}=0.94 f t^{2}$ 
Wing volume

$v=A_{a f} b=\left(6.4839 \frac{1}{144}\right)\left(16.813 * \frac{1}{12}\right)=0.0630 f t^{3}$

Solid blockage correction (multiplied by $1 / 2$ because the model is half of a wing)

$\varepsilon_{s b}=\frac{1}{2}\left(\frac{K \tau v}{C^{3 / 2}}\right)=\frac{1}{2}\left(\frac{(1.04)(0.88)\left(63.7884 * 10^{-3}\right)}{4^{3 / 2}}\right)=0.0073$

Wake blockage correction $(\operatorname{Re}=100,000 \alpha=10$. Assuming no separation)

$\varepsilon_{w b}=\frac{S}{4 C} C_{D 0}+\frac{5 S}{2 C}\left(C_{D u}-C_{D I}-C_{D 0}\right)=\frac{1}{2}\left(\frac{1.89}{4(4)} 0.02+0\right)=0.0047$

Dynamic pressure correction due to solid and wake blockage

$K_{\varepsilon}=\left(1+\left(\varepsilon_{s b}+\varepsilon_{w b}\right)\right)^{2}=(1+(0.0073+0.0047))^{2}=1.024$

Lift $(\operatorname{Re}=100,000, \alpha=10)$

$V=-976.2344 * 10^{-6}$ Volts

$L=375.32 \mathrm{~V}+0.7267=(375.32)\left(-976.2344 * 10^{-6}\right)+0.7267=0.360 \mathrm{lb}$

Lift Coefficient $(\operatorname{Re}=100,000, \alpha=10)$

$C_{L}=\frac{L}{q_{\infty} S}=\frac{0.360 * 2}{(0.68256)(1.88)}=0.561$

$\operatorname{Drag}(\operatorname{Re}=100,000, \alpha=10)$

$V=-1.8276 * 10^{-3}$ Volts

$D=375.32 \mathrm{~V}+0.7267=(375.32)\left(-1.8276 * 10^{-3}\right)+0.7267=0.041 \mathrm{lb}$ 
Drag Coefficient $(\operatorname{Re}=100,000, \alpha=10)$

$C_{D}=\frac{D}{q_{\infty} S}=\frac{0.041 * 2}{(0.68256)(1.88)}=0.064$

Non-dimensional Fequency, $F^{+}(R e=100,000, f=100 \mathrm{~Hz})$

$F+=\frac{f c}{u}=\frac{100 *\left(\frac{8}{12}\right)}{24}=2.778$ 


\section{B Uncertainty Analysis}

\section{B.1 Pressure Coefficient}

The pressure scanner used to acquire pressure data for the $\mathrm{Cp}$ calculation used two sets of pressure transducers. Ports 1-32 used pressure transducers with a range of $+-2.491 \mathrm{KPa}(10$ $\mathrm{inH}_{2} \mathrm{O}$ ) and ports 33-64 used transducers with a range of +- 249.1 $\mathrm{Pa}$ (1 psid). Rows $\mathrm{B}, \mathrm{C}, \mathrm{E}$, and the first 4 ports of row A (starting at the leading edge) used transducers 1-32. Rows D, F, the tunnel static pressure, and the tunnel total pressure used ports 33-64. The error in the pressure values consisted of random and systematic error, which were both included in the error calculation. The random error was found by taking the standard deviation of five consecutive scans consisting of 400 frames each with the tunnel off. The systematic error consists of the drift and zero offset after CALZ is commanded to the system. The zero offset error was eliminated by taking a reading with the tunnel off after calibration and using the non-zero pressure readings as tares. The drift was found by measuring how much the "tunnel off" pressure values drifted at the end of the test session. The following sample error calculation is for the Row $\mathrm{C}$ port at $\mathrm{x} / \mathrm{c}=2.1 \%$ at $\alpha=10$ and $\operatorname{Re}=100,000$.

$$
\begin{gathered}
\delta p_{i}=\delta p_{i, \text { random }}+\delta p_{i, \text { systematic }} \\
=2.0637 * 10^{-6}+1.3949 * 10^{-4}=1.4156 * 10^{-4} \\
\delta p_{\text {tunnel }}=\delta p_{\text {tunnel,random }}+\delta p_{\text {tunnel,systematic }} \\
=9.6732 e-06+4.9372 * 10^{-5}=5.9045 * 10^{-5} \\
\delta p_{t, \text { tunnel }}=\delta p_{t, \text { tunnel,random }}+\delta p_{t, \text { tunnel,systematic }} \\
=8.7891 * 10^{-5}+6.1199 * 10^{-5}=6.9989 * 10^{-5}
\end{gathered}
$$




$$
\begin{aligned}
& \frac{\partial C_{p}}{\partial p_{i}}=\frac{1}{K_{\varepsilon} K_{q}\left(p_{t, \text { tunnel }}-p_{\text {tunnel }}\right)} \\
& =\frac{1}{(1.024)(1.0015)(-0.0031-(-0.0069))}=255.3314 \\
& \frac{\partial C_{p}}{\partial p_{\text {tunnel }}}=\frac{-K_{p}}{K_{\varepsilon} K_{q}\left(p_{t, \text { tunnel }}-p_{\text {tunnel }}\right)}+\frac{\left(p_{i}-K_{p} p_{\text {tunnel }}\right)}{K_{\varepsilon} K_{q}\left(p_{t, \text { tunnel }}-p_{\text {tunnel }}\right)^{2}} \\
& =\frac{-1.0119}{(1.024)(1.0015)(-0.0069-(-0.0031))}+\frac{(-0.0153)-1.0119(-0.0031)}{(1.024)(1.0015)(-0.0069-(-0.0031))^{2}} \\
& =-561.6879 \\
& \frac{\partial C_{p}}{\partial p_{t, \text { tunnel }}}=\frac{K_{p} p_{\text {tunnel }}-p_{i}}{K_{\varepsilon} K_{q}\left(p_{\text {tunnel }}-p_{t, \text { tunnel }}\right)^{2}} \\
& =\frac{1.0119(-0.0069)-(-0.0153)}{(1.024)(1.0015)(-0.0069-(-0.0031))^{2}}=558.2048 \\
& \delta C_{p}=\sqrt{\left(\frac{\partial C_{p}}{\partial p_{i}} \delta p_{i}\right)^{2}+\left(\frac{\partial C_{p}}{\partial p_{\text {tunnel }}} \delta p_{\text {tunnel }}\right)^{2}+\left(\frac{\partial C_{p}}{\partial p_{t, \text { tunnel }}} \delta p_{t, \text { tunnel }}\right)^{2}} \\
& =\sqrt{\left((255.3)\left(1.42 * 10^{-4}\right)\right)^{2}+\left((-816.6)\left(5.90 * 10^{-5}\right)\right)^{2}+\left((558.2)\left(7.00 * 10^{-5}\right)\right)^{2}} \\
& =0.072
\end{aligned}
$$

The error bars for Rows A \& B can be seen in the following figure. 


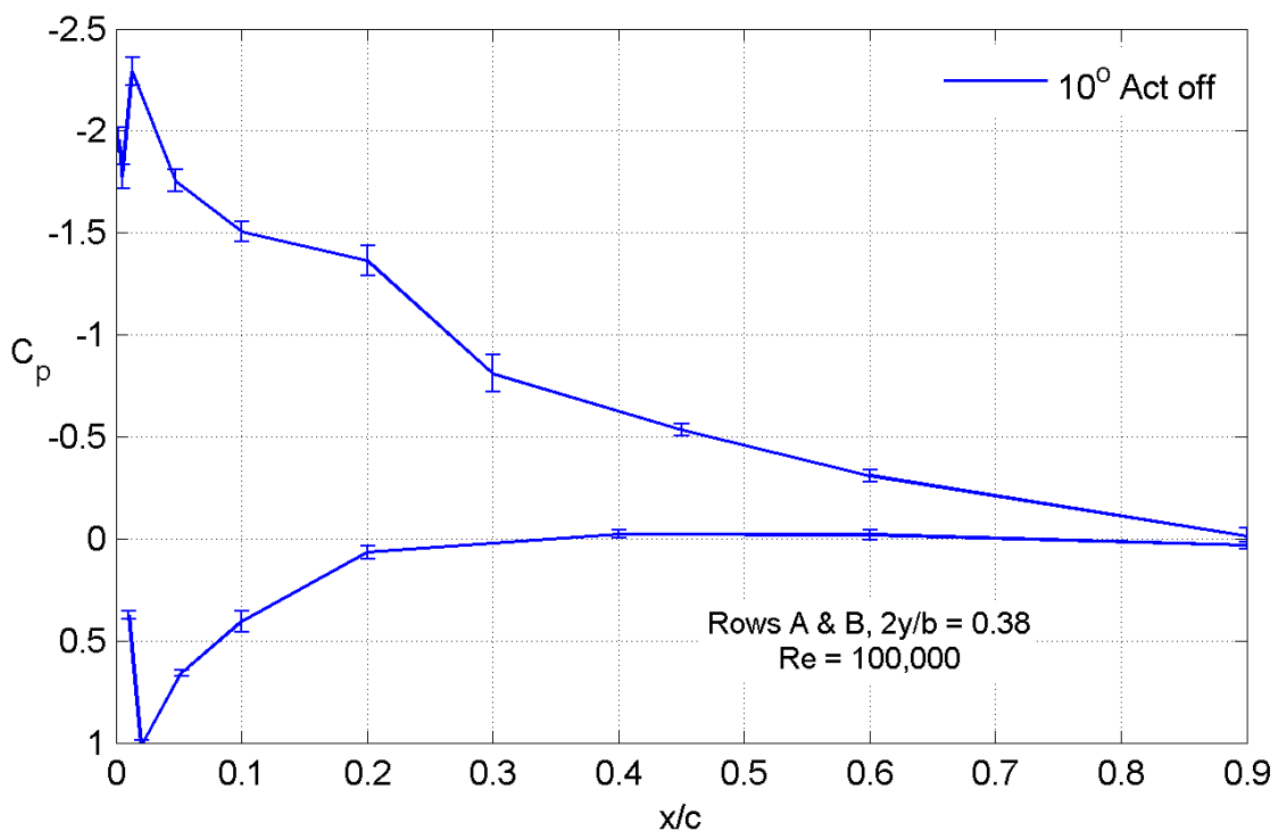

Figure 7.1 Error bars for Rows A \& B: $\operatorname{Re}=100,000, \alpha=10 \mathrm{deg}$.

\section{B.2 Sectional Lift Coefficient}

Assuming that the $\mathrm{x}$ and $\mathrm{y}$ locations are accurate. The calculation was done for Rows A \& $\mathrm{B}$ at $\operatorname{Re}=100,000$ and $\alpha=10$ degrees. Because there are 21 panels, only a plot of the results is shown instead of tabulating the individual values. The pressure drag coefficient was not used in this Thesis and is not included in the analysis. Figure 7.2 shows the error bars on the lift coefficient. In Figure 7.2(b), the error was smaller than the markers.

$c_{n}=-\sum_{\text {pannels }}\left(\frac{C_{p i}+C_{p i+1}}{2}\right)\left(\frac{x_{i+1}}{c}-\frac{x_{i}}{c}\right)$

Where,

$C_{N_{i}}=\left(\frac{C_{p i}+C_{p i+1}}{2}\right)\left(\frac{x_{i+1}}{c}-\frac{x_{i}}{c}\right)$

$\frac{\partial C_{N_{i}}}{\partial C_{p i}}=\frac{1}{2}\left(\frac{x_{i+1}}{c}-\frac{x_{i}}{c}\right)$ 


$$
\begin{aligned}
& \frac{\partial C_{N_{i}}}{\partial C_{p i+1}}=\frac{1}{2}\left(\frac{x_{i+1}}{c}-\frac{x_{i}}{c}\right) \\
& \delta C_{N_{i}}=\sqrt{\left(\frac{\partial C_{N_{i}}}{C_{p i}} \delta C_{p i}\right)^{2}+\left(\frac{\partial C_{N_{i}}}{C_{p i+1}} \delta C_{p i+1}\right)^{2}} \\
& \delta C_{N_{i}}=\sqrt{\left(\frac{\left.\delta C_{p i}\left(\frac{x_{i+1}}{c}-\frac{x_{i}}{c}\right)\right)^{2}+\left(\frac{\delta C_{p i+1}}{2}\left(\frac{x_{i+1}}{c}-\frac{x_{i}}{c}\right)\right)^{2}}{\sum_{\text {pannels }}\left(\delta C_{N_{i}}\right)^{2}}\right.} \\
& \delta c_{n}=\sqrt{ }
\end{aligned}
$$

Similarly,

$$
\begin{aligned}
& c_{a}=\sum_{\text {pannels }}\left(\frac{C_{p i}+C_{p i+1}}{2}\right)\left(\frac{y_{i+1}}{c}-\frac{y_{i}}{c}\right) \\
& \delta C_{A_{i}}=\sqrt{\left(\frac{\delta C_{p i}}{2}\left(\frac{y_{i+1}}{c}-\frac{y_{i}}{c}\right)\right)^{2}+\left(\frac{\delta C_{p i+1}}{2}\left(\frac{y_{i+1}}{c}-\frac{y_{i}}{c}\right)\right)^{2}} \\
& \delta c_{a}=\sqrt{\sum_{p a n n e l s}\left(\delta C_{A_{i}}\right)^{2}} \\
& \delta \alpha=0.1 \text { degrees } \\
& c_{l}=c_{n} \cos \alpha-c_{a} \sin \alpha \\
& \frac{\partial c_{l}}{\partial \alpha}=-c_{n} \sin \alpha-c_{a} \cos \alpha \\
& \frac{\partial c_{l}}{\partial c_{n}}=\cos \alpha \\
& \frac{\partial c_{l}}{\partial c_{a}}=-\sin \alpha \\
& \delta c_{l}=\sqrt{\left(\frac{\partial c_{l}}{\partial \alpha} \delta \alpha\right)^{2}+\left(\frac{\partial c_{l}}{\partial c_{n}} \delta c_{n}\right)^{2}+\left(\frac{\partial c_{l}}{\partial c_{a}} \delta c_{a}\right)^{2}}
\end{aligned}
$$



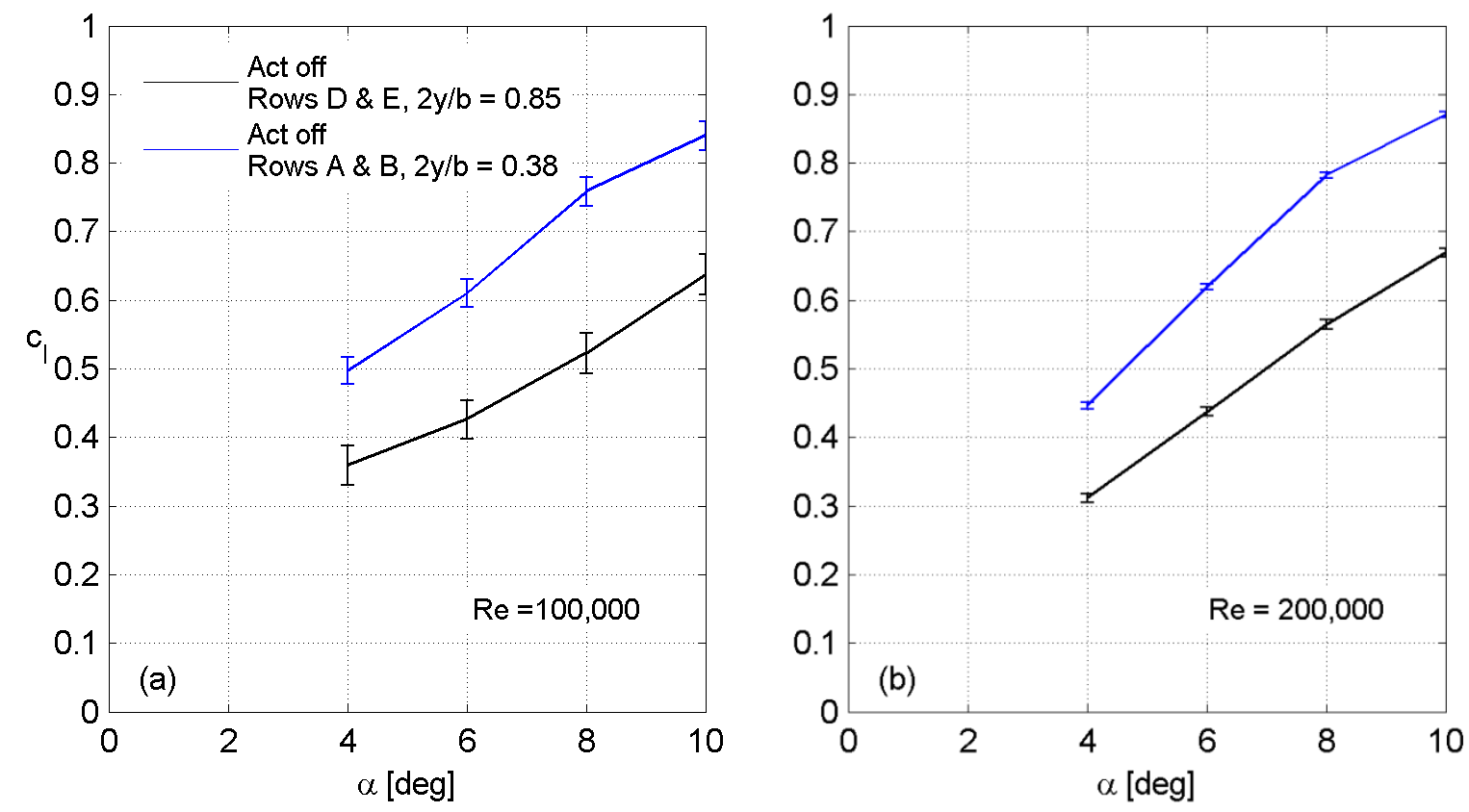

Figure 7.2 Lift coefficient error bars

\section{B.3 Lift and Drag Coefficient Error}

The accuracy of the load cell is $0.15 \%$ FSO. For this load cell, the FSO is $1 \mathrm{~kg}(2.2 \mathrm{lb})$ so the accuracy is $0.0015 \mathrm{~kg}(0.0033 \mathrm{lb})$. Assuming the freestream dynamic pressure and planform area are accurate, the error in the lift coefficient is

$\delta C_{L}=\frac{\delta L}{q_{\infty} S}$

Where,

$\delta L=0.0033$

For the drag coefficient the error is

$\delta C_{D}=\frac{\delta D}{q_{\infty} S}$

Where,

$\delta D=0.0033$

The following sample calculation shows $\delta C_{D}$ for $R e=100,000$ and $\alpha=4$. 
$\delta C_{D}=\frac{\delta D}{q_{\infty} S}=\frac{0.0033}{0.68256 * 0.94}=0.0051$

Figure 7.3 shows error bars on the lift and drag for the baseline case
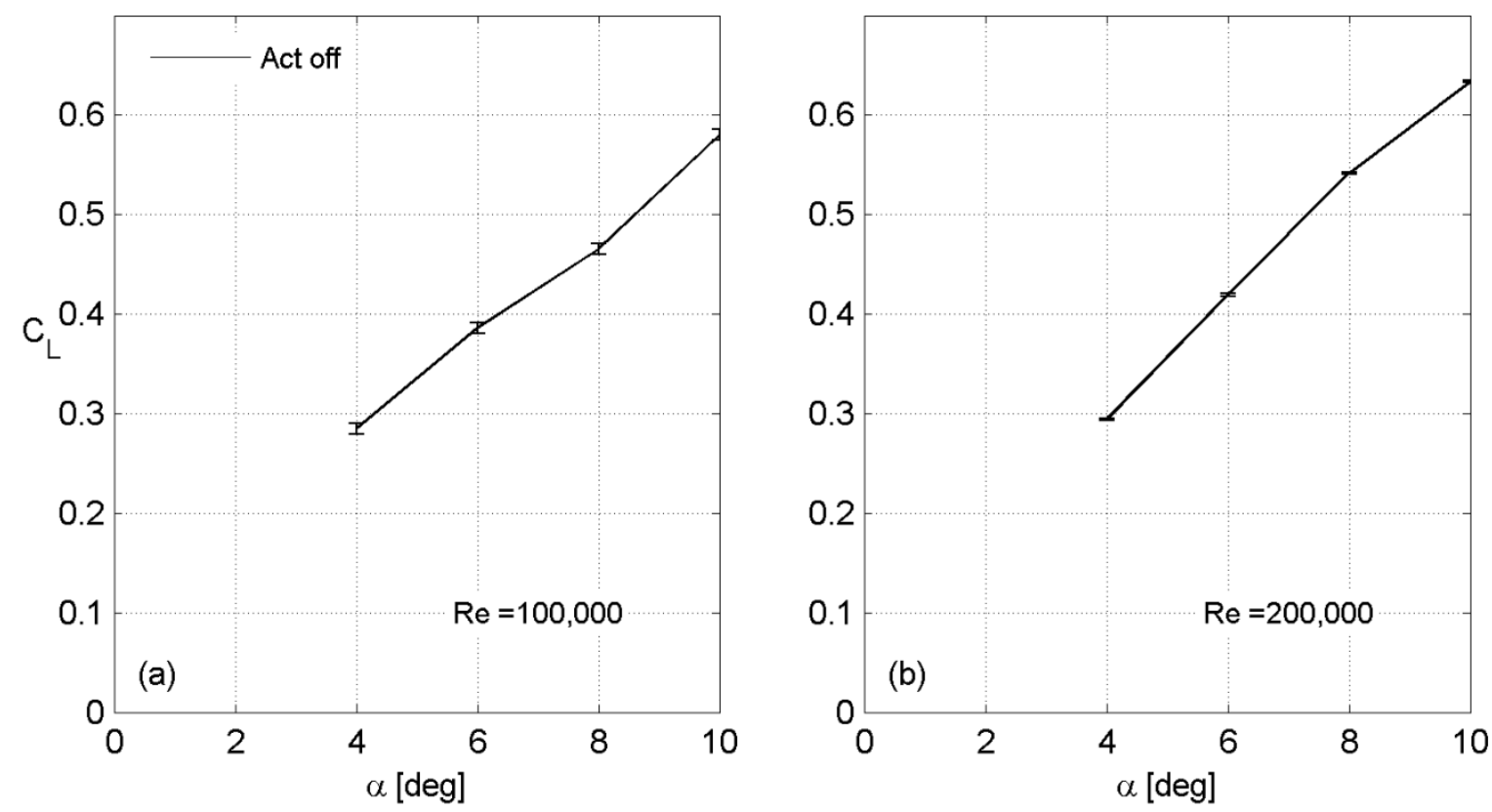

Figure 7.3 Lift curve with error bars

The error in the lift coefficient is very small. The error in the drag coefficient is shown in Figure 7.4 

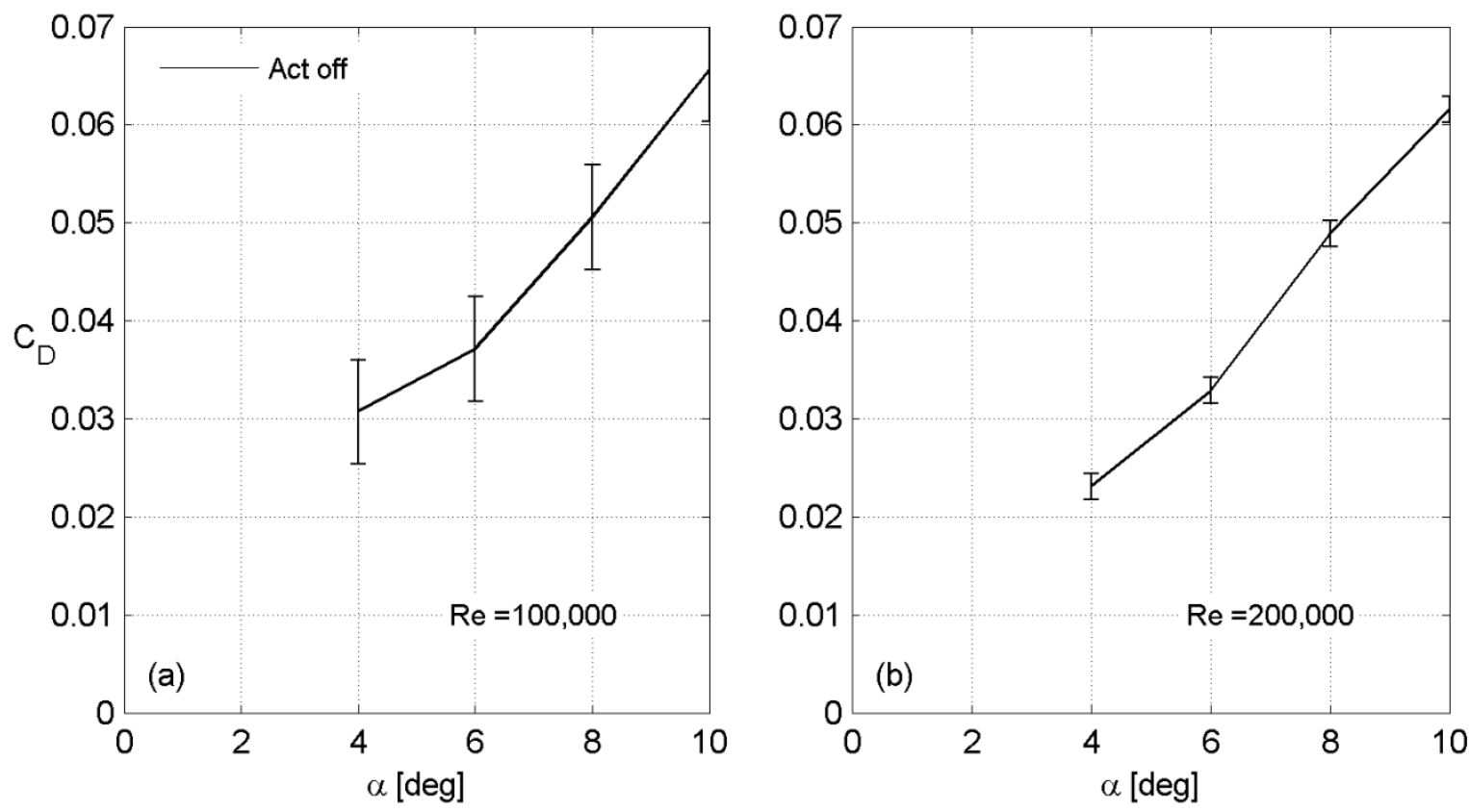

Figure 7.4 Drag curve with error bars

The error in the drag coefficient at $\operatorname{Re}=100,000$ is very large. This is because the magnitude of the drag was very low so the $0.0015 \mathrm{~kg}(0.0033 \mathrm{lb})$ error in the load cell had a large impact. The following sample calculation shows $\delta C_{D}$ for $\operatorname{Re}=100,000$ and $\alpha=4$.

$\delta C_{D}=\frac{\delta D}{q_{\infty} S}=\frac{0.0033}{0.68256 * 0.94}=0.0051$

At $\operatorname{Re}=200,000$ the error was much lower because the drag increased significantly. It is possible that the error was smaller than what is shown in Figure 7.4. The accuracy of the load cell used in the error analysis was the worst case error as specified by the manufacturer. Durring testing, for each angle of attack there were several repeated points taken to verify the repeatability of the data. A sample error plot for the $\mathrm{R}=100,000$ baseline drag using the standard deviation between the repeat values is shown in Figure 7.5. The error bars are much smaller than they were in Figure 7.4. This shows that it is quite possible that there was less error than what was specified as the worst case by the manufacturer. 


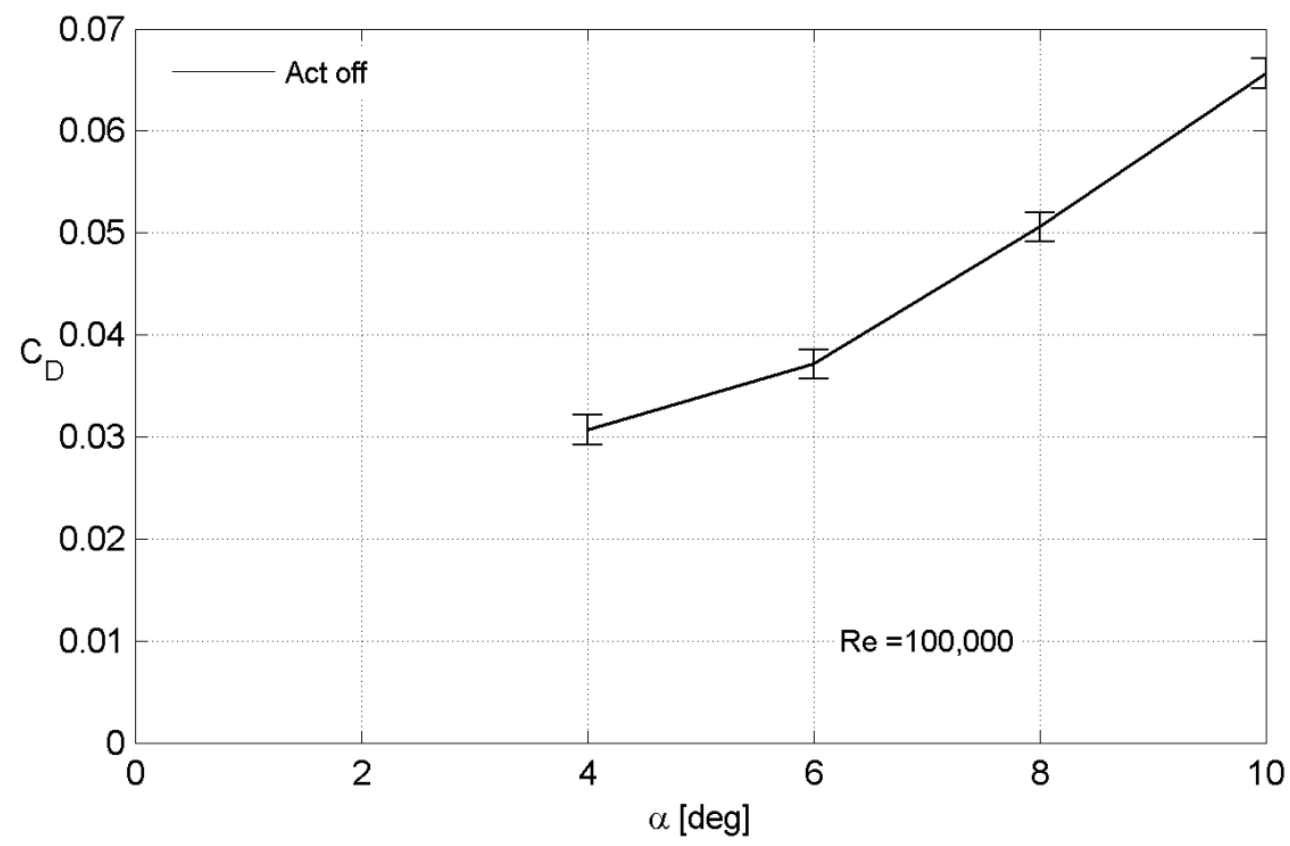

Figure 7.5 Drag curve with error bars showing error calculate using the standard deviation of repeat load cell readings

\section{B.4 Momentum Coefficient Error}

The error in the momentum coefficient comes from a few sources. The first is human error in the positioning of the hotwire since the hotwire was placed in several locations which were measured with a caliper and micrometer depending on the application. Another source of error comes from the fact that the hotwire could not be placed at the optimal location just inside the jet. This could not be done because if the hotwire is positioned too close it could easily bump into the slit and break. Another source of error is the accuracy of the anemometer itself. The error at each calibration point was recorded by the anemometer. The anemometer error can be using

$u_{j}=A V^{3}+B V^{2}+C V+D$

Where $u_{j}$ is the instantaneous jet velocity, $V$ is the voltage, and $A-D$ are the $4^{\text {th }}$ order polynomial constants. Looking at Figure 5.1, the error in the voltage is about 1 percent. The error can be calculated as follows.

$\frac{\partial u_{j}}{\partial V}=3 A V^{2}+2 B V+C$

Where, 


$$
\begin{aligned}
& A=-108.925 \\
& B=360.350 \\
& C=-412.726 \\
& D=-8.535
\end{aligned}
$$

\section{Therefore,}

$$
\begin{aligned}
& \delta u_{j}=\sqrt{\left[\left(3 A V^{2}+2 B V+C\right) \delta V\right]^{2}} \\
& \text { Where }
\end{aligned}
$$

$$
\delta V=0.01 \mathrm{~V}
$$




\section{LabVIEW Block Diagram}

The labview block digram is shown for the LabVIEW VI used to control the experiment

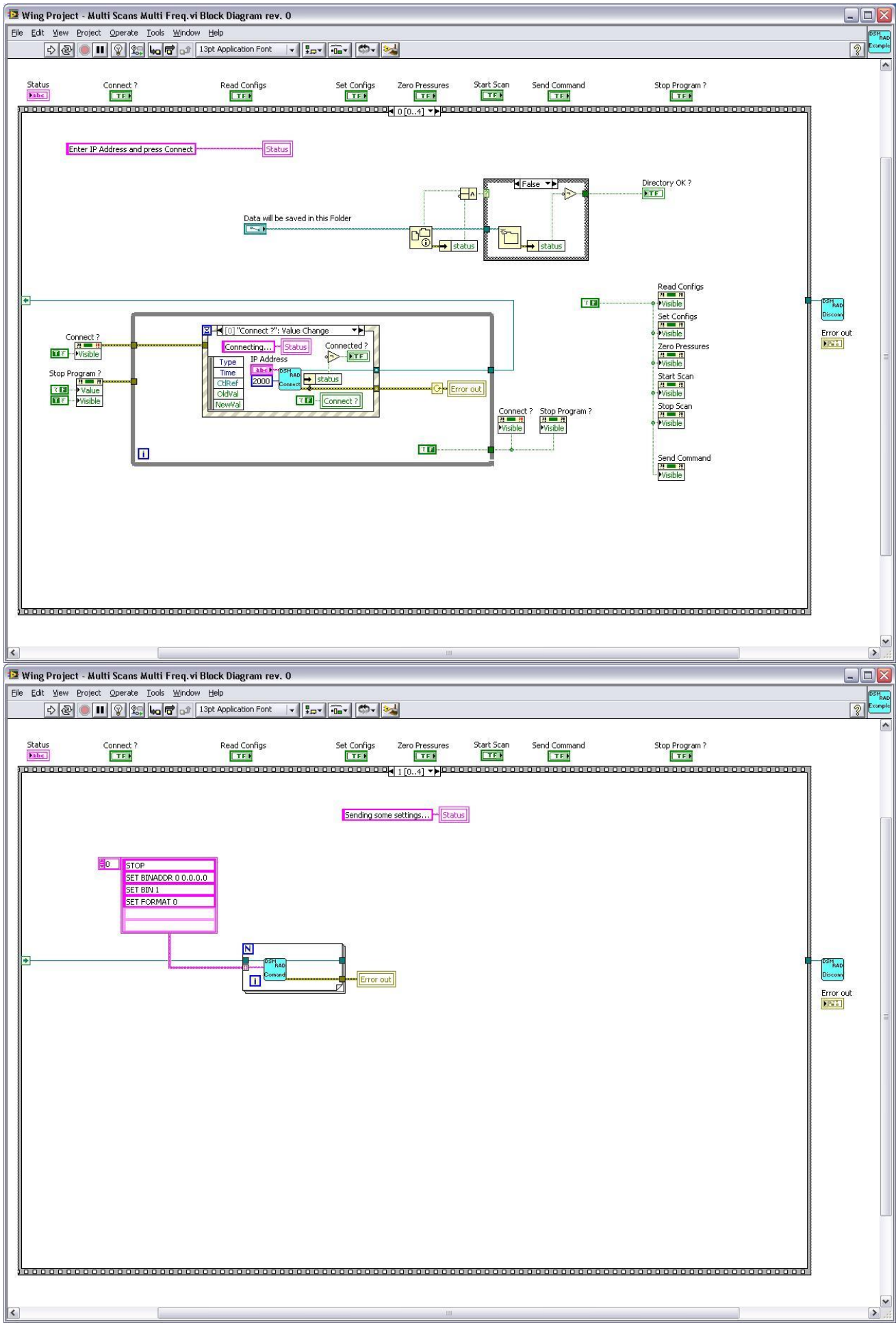




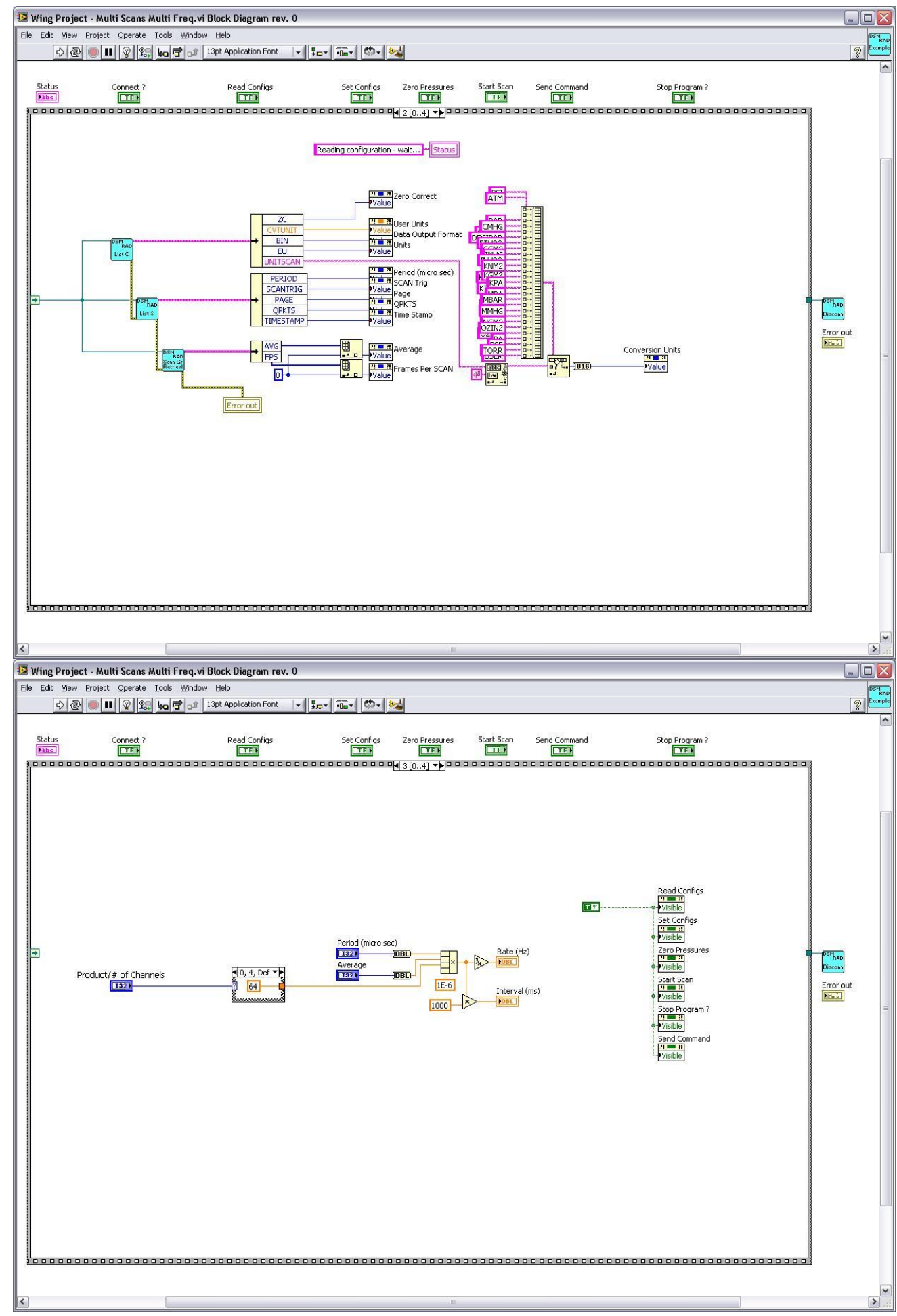




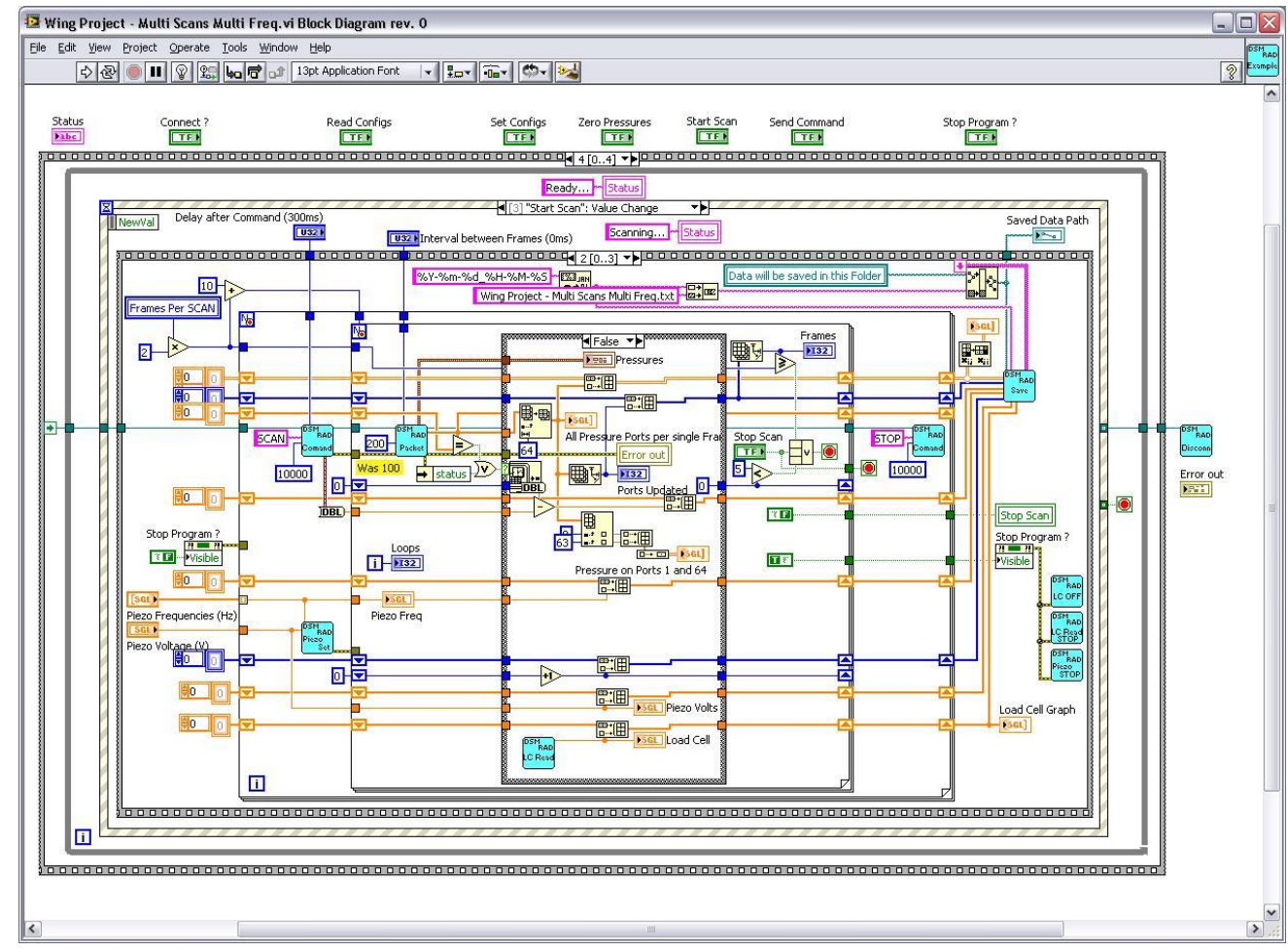

